IEEE $X$ plore
Access provided by:

Australian National University

Sign Out

- IEEE

Advanced Search | Preferences | Search Tips | More Search Options -

Browse Conference Publications > Acoustics, Speech and Signal ...

\title{
Forensic voice comparison with secular shibboleths - A hybrid? Page Help fused gmm-multivariate likelihood ratio-based approach using alveolo-palatal fricative cepstral spectra
}

This paper appears in:

Acoustics, Speech and Signal Processing (ICASSP), 2011 IEEE

International Conference on

Date of Conference: 22-27 May 2011

Author(s): Rose, P.

On Page(s): 5900 - 5903

Product Type: Conference Publications

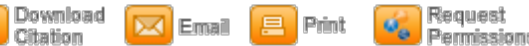

\section{ABSTRACT}

The suitability of voiceless fricative spectra for forensic voice comparison is explored within a Likelihood Ratiobased framework. Non-contemporaneous landline telephone recordings of 99 male Japanese speakers are compared using only tokens of their voiceless alveolo-patalal fricative [ç]. A subset of mean-cepstrallysubtracted LPC CCs from the fricative spectrum from dc to $5 \mathrm{kHz}$ is used. GMM/UBM and multivariate likelihood ratios are extracted for the 99 target and 4851 non-target trials, and fused with logistic regression. An EER of $7.4 \%$ and log-LR cost of 0.26 is demonstrated. It is concluded that the [ç] spectrum does have some individualising potential.

\section{INDEX TERMS}

\section{- IEEE Terms}

Cavity resonators, Cepstral analysis, Forensics, Speaker recognition, Speech, Tongue

\section{- I NSPEC}

\section{- Controlled I ndexing}

maximum likelihood estimation, speaker recognition

\section{- Non Controlled I ndexing}

LPC CC , UBM , alveolo-palatal fricative cepstral spectra , forensic voice comparison , fricative spectrum , fused GMM-multivariate likelihood ratio-based approach, noncontemporaneous landline telephone recordings , secular shibboleths

\section{- Author Keywords}

Forensic Voice Comparison, GMM/UBM , Multivariate Likelihood Ratio, cepstrum , coronal fricative spectra

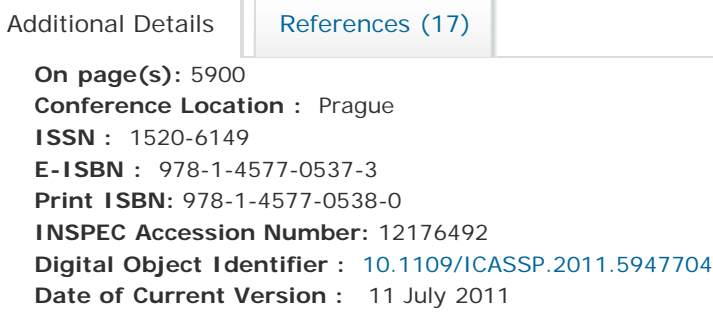




\section{IEEE Account}

* Change Username/Password

* Update Address

\section{Purchase Details}

Payment Options

* Order History

* Access Purchased Documents
Profile I nformation

* Communications Preferences

- Profession and Education

* Technical Interests

\section{Need Help?}

US \& Canada: +1 8006784333

Worldwide: +1 7329810060

Contact \& Support

About IEeE Xplore | Contact | Help | Privacy \& Security | Terms of Use | Nondiscrimination Policy | Accessibility | Site Map 


\section{IEEE International Conference on Acoustics, Speech and Signal Processing}

\section{(ICASSP 2011)}

\section{Prague, Czech Republic 22 - 27 May 2011}

Pages 1-844 
Copyright (C) 2011 by the Institute of Electrical and Electronic Engineers, Inc All Rights Reserved

Copyright and Reprint Permissions: Abstracting is permitted with credit to the source. Libraries are permitted to photocopy beyond the limit of U.S. copyright law for private use of patrons those articles in this volume that carry a code at the bottom of the first page, provided the per-copy fee indicated in the code is paid through Copyright Clearance Center, 222 Rosewood Drive, Danvers, MA 01923.

For other copying, reprint or republication permission, write to IEEE Copyrights Manager, IEEE Service Center, 445 Hoes Lane, Piscataway, NJ 08854. All rights reserved.

***This publication is a representation of what appears in the IEEE Digital Libraries. Some format issues inherent in the e-media version may also appear in this print version.

$\begin{array}{ll}\text { IEEE Catalog Number: } & \text { CFP11ICA-PRT } \\ \text { ISBN 13: } & 978-1-4577-0538-0 \\ \text { ISSN: } & 1520-6149\end{array}$

\section{Additional Copies of This Publication Are Available From:}

Curran Associates, Inc

57 Morehouse Lane

Red Hook, NY 12571 USA

Phone: (845) 758-0400

Fax: $\quad$ (845) 758-2633

E-mail:_curran@proceedings.com

Web: www.proceedings.com 


\section{TABLE OF CONTENTS}

\section{AASP-L1: ACOUSTIC SOURCE SEPARATION I}

AASP-L1.1: COMBINING HMM-BASED MELODY EXTRACTION AND NMF-BASED SOFT

MASKING FOR SEPARATING VOICE AND ACCOMPANIMENT FROM MONAURAL AUDIO

Yun Wang, Zhijian Ou, Tsinghua University, China

AASP-L1.2: ADAPTATION OF SOURCE-SPECIFIC DICTIONARIES IN NON-NEGATIVE

MATRIX FACTORIZATION FOR SOURCE SEPARATION

Xabier Jaureguiberry, Pierre Leveau, Simon Maller, Juan José Burred, Audionamix, France

AASP-L1.3: AN ACOUSTICALLY-MOTIVATED SPATIAL PRIOR FOR UNDER-DETERMINED 9 REVERBERANT SOURCE SEPARATION

Ngoc Q. K. Duong, Emmanuel Vincent, Rémi Gribonval, INRIA / Centre de Rennes - Bretagne Atlantique, France

AASP-L1.4: RESOLVING FD-BSS PERMUTATION FOR ARBITRARY ARRAY IN PRESENCE

OF SPATIAL ALIASING

Jani Even, Norihiro Hagita, ATR, Intelligent Robotics and Communication Laboratories, Japan

AASP-L1.5: A NON-NEGATIVE APPROACH TO SEMI-SUPERVISED SEPARATION OF 17 SPEECH FROM NOISE WITH THE USE OF TEMPORAL DYNAMICS

Gautham J. Mysore, Adobe Systems Inc., United States; Paris Smaragdis, University of Illinois Urbana-Champaign, United States

AASP-L1.6: ITAKURA-SAITO NONNEGATIVE MATRIX FACTORIZATION WITH GROUP 21 SPARSITY

Augustin Lefevre, Francis Bach, Ecole Normale Superieure, France; Cédric Févotte, CNRS LTCI / Télécom ParisTech, France

\section{AASP-L2: MUSIC SIGNAL PROCESSING I}

AASP-L2.1: MULTIPITCH ESTIMATION BY JOINT MODELING OF HARMONIC AND TRANSIENT SOUNDS

Jun Wu, The University of Tokyo, Japan; Emmanuel Vincent, INRIA, France; Stanislaw Raczynski, Takuya Nishimoto, Nobutaka Ono, Shigeki Sagayama, The University of Tokyo, Japan

AASP-L2.2: FREQUENCY SELECTIVE PITCH TRANSPOSITION OF AUDIO SIGNALS

Sascha Disch, Fraunhofer Institute for Integrated Circuits (IIS), Germany; Bernd Edler, International Audio Laboratories

Erlangen, Germany

AASP-L2.3: IMPROVING MELODY EXTRACTION USING PROBABILISTIC LATENT 33 COMPONENT ANALYSIS

Jinyu Han, Northwestern University, United States; Ching-Wei Chen, Gracenote, United States

AASP-L2.4: POLYPHONIC MUSIC TRANSCRIPTION USING NOTE ONSET AND OFFSET 37 DETECTION

Emmanouil Benetos, Simon Dixon, Queen Mary University of London, United Kingdom

AASP-L2.5: AUTOMATIC MUSICAL THUMBNAILING BASED ON AUDIO OBJECT 41 LOCALIZATION AND ITS EVALUATION

Hiroyuki Nawata, Noriyoshi Kamado, Hiroshi Saruwatari, Kiyohiro Shikano, Nara Institute of Science and Technology, Japan

AASP-L2.6: SCORE INFORMED AUDIO SOURCE SEPARATION USING A PARAMETRIC .45 MODEL OF NON-NEGATIVE SPECTROGRAM

Romain Hennequin, Bertrand David, Roland Badeau, Institut TELECOM / TELECOM ParisTech, France 
AASP-L3.1: EFFICIENT RANGE EXTRAPOLATION OF HEAD-RELATED IMPULSE

RESPONSES BY WAVE FIELD SYNTHESIS TECHNIQUES

Sascha Spors, Jens Ahrens, Deutsche Telekom Laboratories, Germany

AASP-L3.2: EFFICIENCY EVALUATION AND ORTHOGONAL BASIS DETERMINATION IN 53 FUNCTIONAL HRTF MODELING

Mengqiu Zhang, Rodney A. Kennedy, Thushara D. Abhayapala, Australian National University, Australia

AASP-L3.3: SPATIAL SOUND REPRODUCTION SYSTEMS USING HIGHER ORDER 57 LOUDSPEAKERS

Mark Poletti, Industrial Research Ltd, New Zealand; Thushara D. Abhayapala, Australian National University, Australia

AASP-L3.4: CONVERTING 5.1 AUDIO RECORDINGS TO B-FORMAT FOR DIRECTIONAL

AUDIO CODING REPRODUCTION

Mikko-Ville Laitinen, Ville Pulkki, Aalto University, Finland

AASP-L3.5: AN ANALYTICAL APPROACH TO LOCAL SOUND FIELD SYNTHESIS USING 65 LINEAR ARRAYS OF LOUDSPEAKERS

Jens Ahrens, Sascha Spors, Deutsche Telekom Laboratories, Germany

AASP-L3.6: A METHODOLOGY FOR EVALUATING THE ACCURACY OF WAVE FIELD RENDERING TECHNIQUES

Antonio Canclini, Politecnico di Milano, Italy; Paolo Annibale, University Erlangen-Nuremberg, Germany; Fabio Antonacci, Augusto Sarti, Politecnico di Milano, Italy; Rudolf Rabenstein, University Erlangen-Nuremberg, Germany; Stefano Tubaro, Politecnico di Milano, Italy

\section{AASP-L4: ECHO CANCELLATION}

AASP-L4.1: A PROPORTIONATE ADAPTIVE ALGORITHM WITH VARIABLE PARTITIONED

BLOCK LENGTH FOR ACOUSTIC ECHO CANCELLATION

Pradeep Loganathan, Emanuel A.P. Habets, Patrick Naylor, Imperial College London, United Kingdom

AASP-L4.2: AN EFFICIENT VARIABLE STEP-SIZE PROPORTIONATE AFFINE PROJECTION 77 ALGORITHM

Constantin Paleologu, University Politehnica of Bucharest, Romania; Jacob Benesty, University of Quebec, Canada; Felix Albu,

Silviu Ciochina, University Politehnica of Bucharest, Romania

AASP-L4.3: RELATIVE PROPORTIONATE NLMS: IMPROVING CONVERGENCE FOR 81 ACOUSTIC CHANNEL IDENTIFICATION

Tao Yu, John Hansen, The University of Texas at Dallas, United States

AASP-L4.4: FOURIER EXPANSION OF HAMMERSTEIN MODELS FOR NONLINEAR 85 ACOUSTIC SYSTEM IDENTIFICATION

Sarmad Malik, Gerald Enzner, Ruhr-Universität Bochum, Germany

AASP-L4.5: ROBUST AND LOW-COST CASCADED NON-LINEAR ACOUSTIC ECHO 89 CANCELLATION

Moctar Mossi Idrissa, Christelle Yemdji, Nicholas Evans, EURECOM, France; Christophe Beaugeant, Philippe Degry, Infineon, France

AASP-L4.6: SPATIO-TEMPORAL SIGNAL PREPROCESSING FOR MULTICHANNEL 93 ACOUSTIC ECHO CANCELLATION

Karim Helwani, Sascha Spors, Herbert Buchner, Deutsche Telekom Laboratories / Technische Universität Berlin, Germany 


\section{AASP-L5: MICROPHONE ARRAY SIGNAL PROCESSING}

AASP-L5.1: BROADBAND DIRECTION ESTIMATION METHOD UTILIZING COMBINED 97 PRESSURE AND ENERGY GRADIENTS FROM OPTIMIZED MICROPHONE ARRAY

Jukka Ahonen, Ville Pulkki, Aalto University, Finland

AASP-L5.2: DESIGN OF ROBUST STEERABLE BROADBAND BEAMFORMERS 101 INCORPORATING MICROPHONE GAIN AND PHASE ERROR CHARACTERISTICS Chiong Ching Lai, Sven Nordholm, Yee Hong Leung, Curtin University, Australia

AASP-L5.3: DIRECTION-OF-ARRIVAL ESTIMATION USING ACOUSTIC VECTOR SENSORS 105 IN THE PRESENCE OF NOISE

Dovid Levin, Bar-Ilan University, Israel; Emanuel A.P. Habets, Imperial College London, United Kingdom; Sharon Gannot, BarIlan University, Israel

AASP-L5.4: MICROPHONE POSITION OPTIMIZATION FOR PLANAR SUPERDIRECTIVE 109 BEAMFORMING

Ina Kodrasi, University of Oldenburg, Germany; Thomas Rohdenburg, Fraunhofer IDMT, Germany; Simon Doclo, University of Oldenburg, Germany

AASP-L5.5: JOINT DOA AND TDOA ESTIMATION FOR 3D LOCALIZATION OF REFLECTIVE 113 SURFACES USING EIGENBEAM MVDR AND SPHERICAL MICROPHONE ARRAYS Haohai Sun, Norwegian University of Science and Technology, Norway; Edwin Mabande, Konrad Kowalczyk, Walter Kellermann, University of Erlangen-Nuremberg, Germany

AASP-L5.6: ROBUST LOCALIZATION OF MULTIPLE SOURCES IN REVERBERANT 117 ENVIRONMENTS USING EB-ESPRIT WITH SPHERICAL MICROPHONE ARRAYS

Haohai Sun, Norwegian University of Science and Technology, Norway; Heinz Teutsch, Avaya Labs, Germany; Edwin Mabande, Walter Kellermann, University of Erlangen-Nuremberg, Germany

\section{AASP-P1: LOUDSPEAKER AND MICROPHONE ARRAY SIGNAL PROCESSING}

AASP-P1.1: PERFORMANCE ANALYSIS OF A RANDOMLY SPACED WIRELESS 121 MICROPHONE ARRAY

Shmulik Markovich Golan, Sharon Gannot, Bar-Ilan University, Israel; Israel Cohen, Technion / Israel Institute of Technology, Israel

AASP-P1.2: A GENERALIZED DESIGN METHOD FOR DIRECTIVITY PATTERNS OF 125 SPHERICAL MICROPHONE ARRAYS

Enzo De Sena, Huseyin Hacihabiboglu, Zoran Cvetkovic, King's College London, United Kingdom

AASP-P1.3: SIMULATING ROOM IMPULSE RESPONSES FOR SPHERICAL MICROPHONE 129 ARRAYS

Daniel Jarrett, Emanuel A.P. Habets, Mark Thomas, Patrick Naylor, Imperial College London, United Kingdom

AASP-P1.4: RESOLVING SPATIAL SAMPLING EFFECTS IN PARAMETRIC DIRECTIONAL 133 FILTERING

Markus Kallinger, Michael Buerger, Oliver Thiergart, Fabian Kuech, Dirk Mahne, Fraunhofer Institute for Integrated Circuits (IIS), Germany

AASP-P1.5: A DATA-DRIVEN POST-FILTER DESIGN BASED ON SPATIALLY AND 137 TEMPORALLY SMOOTHED A PRIORI SNR

Huajun Yu, Tim Fingscheidt, TU Braunschweig, Germany

AASP-P1.6: DESIGN OF MULTIPOLE LOUDSPEAKER ARRAY BASED ON SPHERICAL 141 HARMONIC EXPANSION

Yoichi Haneda, Ken'ichi Furuya, Hiroaki Itou, NTT Corporation, Japan 
AASP-P1.7: A WAVENUMBER-FITTING EXTRAPOLATION METHOD FOR FFT-BASED 145

NEAR-FIELD ACOUSTIC HOLOGRAPHY USING MICROPHONE ARRAY

Benxu Liu, Bremananth Ramachandran, Andy W. H. Khong, Nanyang Technological University, Singapore

AASP-P1.8: APPROXIMATED KERNEL DENSITY ESTIMATION FOR MULTIPLE TDOA 149

DETECTION

Francesco Nesta, Maurizio Omologo, Fondazione Bruno Kessler, Italy

AASP-P1.9: ON 2D LOCALIZATION OF REFLECTORS USING ROBUST BEAMFORMING 153 TECHNIQUES

Edwin Mabande, University of Erlangen-Nuremberg, Germany; Haohai Sun, Norwegian University of Science and Technology,

Norway; Konrad Kowalczyk, Walter Kellermann, University of Erlangen-Nuremberg, Germany

AASP-P1.10: SYNTHESIS OF ICA-BASED METHODS FOR LOCALIZATION OF MULTIPLE 157 BROADBAND SOUND SOURCES

Anthony Lombard, Yuanhang Zheng, Walter Kellermann, University of Erlangen-Nuremberg, Germany

\section{AASP-P2: MUSIC SIGNAL PROCESSING II}

AASP-P2.1: SOUND MORPHING BY FEATURE INTERPOLATION

Marcelo Caetano, Xavier Rodet, Institut de Recherche et Coordination Acoustique/Musique, France

AASP-P2.2: KNOW THY NEIGHBOR: COMBINING AUDIO FEATURES AND SOCIAL TAGS 165 FOR EFFECTIVE MUSIC SIMILARITY

Alexandros Nanopoulos, University of Hildesheim, Germany; Ioannis Karydis, Ionian University, Greece

AASP-P2.3: NONLINEAR AUDIO RECURRENCE ANALYSIS WITH APPLICATION TO GENRE 169 CLASSIFICATION

Joan Serrà, Carlos A. de los Santos, Ralph G. Andrzejak, Universitat Pompeu Fabra, Spain

AASP-P2.4: TIME-CONSTRAINED SEQUENTIAL PATTERN DISCOVERY FOR MUSIC GENRE CLASSIFICATION

Jia-Min Ren, Jyh-Shing Roger Jang, National Tsing Hua University, Taiwan

AASP-P2.5: EVALUATING MUSIC SEQUENCE MODELS THROUGH MISSING DATA.

Thierry Bertin-Mahieux, Graham Grindlay, Columbia University, United States; Ron J. Weiss, New York University, United

States; Daniel P.W. Ellis, Columbia University, United States

AASP-P2.6: TIME-FREQUENCY REASSIGNED FEATURES FOR AUTOMATIC CHORD

RECOGNITION

Maksim Khadkevich, Maurizio Omologo, Fondazione Bruno Kessler, Italy

AASP-P2.7: POLYPHONIC AUDIO-TO-SCORE ALIGNMENT BASED ON BAYESIAN LATENT 185 HARMONIC ALLOCATION HIDDEN MARKOV MODEL

Akira Maezawa, Hiroshi G. Okuno, Tetsuya Ogata, Kyoto University, Japan; Masataka Goto, National Institute of Advanced Industrial Science and Technology, Japan

AASP-P2.8: MODELING MUSICAL ATTRIBUTES TO CHARACTERIZE ENSEMBLE 189 RECORDINGS USING RHYTHMIC AUDIO FEATURES

Jakob Abesser, Fraunhofer IDMT, Germany; Olivier Lartillot, University of Jyväskylä, Finland; Christian Dittmar, Fraunhofer IDMT, Germany; Tuomas Eerola, University of Jyväskylä, Finland; Gerald Schuller, Fraunhofer IDMT, Germany

AASP-P2.9: A UNIFIED APPROACH TO REAL TIME AUDIO-TO-SCORE AND 193 AUDIO-TO-AUDIO ALIGNMENT USING SEQUENTIAL MONTECARLO INFERENCE TECHNIQUES

Nicola Montecchio, University of Padova, Italy; Arshia Cont, Institut de Recherche et Coordination Acoustique/Musique, France

AASP-P2.10: A STATE SPACE MODEL FOR ONLINE POLYPHONIC AUDIO-SCORE 197 ALIGNMENT

Zhiyao Duan, Bryan Pardo, Northwestern University, United States 
AASP-P3.1: GEOMETRIC MULTICHANNEL COMMON SIGNAL SEPARATION WITH

APPLICATION TO MUSIC AND EFFECTS EXTRACTION FROM FILM SOUNDTRACKS

Juan José Burred, Pierre Leveau, Audionamix, France

AASP-P3.2: MULTICHANNEL HARMONIC AND PERCUSSIVE COMPONENT SEPARATION

BY JOINT MODELING OF SPATIAL AND SPECTRAL CONTINUITY

Ngoc Q. K. Duong, INRIA / Centre de Rennes - Bretagne Atlantique, France; Hideyuki Tachibana, The University of Tokyo, Japan; Emmanuel Vincent, INRIA / Centre de Rennes - Bretagne Atlantique, France; Nobutaka Ono, The University of Tokyo, Japan; Rémi Gribonval, INRIA / Centre de Rennes - Bretagne Atlantique, France; Shigeki Sagayama, The University of Tokyo, Japan

AASP-P3.3: INTEGRATING BINAURAL CUES AND BLIND SOURCE SEPARATION METHOD 209 FOR SEPARATING REVERBERANT SPEECH MIXTURES

Atiyeh Alinaghi, Wenwu Wang, Philip J. B. Jackson, University of Surrey, United Kingdom

AASP-P3.4: ONLINE SPEECH SOURCE SEPARATION BASED ON MAXIMUM LIKELIHOOD 213

OF LOCAL GAUSSIAN MODELING

Masahito Togami, Hitachi Ltd., Japan

AASP-P3.5: DEGENERATE UNMIXING ESTIMATION TECHNIQUE USING THE 217 CONSTANT Q TRANSFORM

Zafar Rafii, Bryan Pardo, Northwestern University, United States

AASP-P3.6: A SIMPLE MUSIC/VOICE SEPARATION METHOD BASED ON THE 221 EXTRACTION OF THE REPEATING MUSICAL STRUCTURE

Zafar Rafii, Bryan Pardo, Northwestern University, United States

AASP-P3.7: HYBRID APPROACH FOR MULTICHANNEL SOURCE SEPARATION COMBINING TIME-FREQUENCY MASK WITH MULTI-CHANNEL WIENER FILTER

Shoko Araki, Tomohiro Nakatani, NTT Communication Science Laboratories, Japan

AASP-P3.8: FORMULATIONS AND ALGORITHMS FOR MULTICHANNEL COMPLEX NMF 229

Hiroshi Sawada, Hirokazu Kameoka, Shoko Araki, Naonori Ueda, NTT Corporation, Japan

AASP-P3.9: DISTRIBUTED BLIND SOURCE SEPARATION WITH AN APPLICATION TO 233 AUDIO SIGNALS

Yusuke Hioka, NTT, Japan; W. Bastiaan Kleijn, Victoria University of Wellington, New Zealand

AASP-P3.10: JOINT UNSUPERVISED LEARNING OF HIDDEN MARKOV SOURCE 237 MODELS AND SOURCE LOCATION MODELS FOR MULTICHANNEL SOURCE SEPARATION

Tomohiro Nakatani, Shoko Araki, Takuya Yoshioka, Masakiyo Fujimoto, NTT Corporation, Japan

AASP-P4: ACOUSTIC SOURCE SEPARATION AND NOISE REDUCTION

AASP-P4.1: MULTIMODAL BLIND SOURCE SEPARATION FOR MOVING SOURCES BASED 241 ON ROBUST BEAMFORMING

Syed Mohsen Naqvi, Miao Yu, Jonathon A. Chambers, Loughborough University, United Kingdom

AASP-P4.2: CLUSTERING NMF BASIS FUNCTIONS USING SHIFTED NMF FOR 245 MONAURAL SOUND SOURCE SEPARATION

Rajesh Jaiswal, Derry FitzGerald, Dan Barry, Eugene Coyle, Dublin Institute of Technology, Ireland; Scott Rickard, University College Dublin, Ireland

AASP-P4.3: RECONSTRUCTING COMPLETELY OVERLAPPED NOTES FROM MUSICAL 249 MIXTURES

Jinyu Han, Bryan Pardo, Northwestern University, United States 
NON-NEGATIVE MATRIX FACTORIZATION BASED SINGLE CHANNEL SOUND SOURCE

SEPARATION

Serap Kirbiz, Istanbul Technical University, Turkey; Paris Smaragdis, University of Illinois, United States

AASP-P4.5: MULTICHANNEL NONNEGATIVE TENSOR FACTORIZATION WITH 257 STRUCTURED CONSTRAINTS FOR USER-GUIDED AUDIO SOURCE SEPARATION

Alexey Ozerov, INRIA / Centre de Rennes - Bretagne Atlantique, France; Cédric Févotte, CNRS LTCI / Télécom ParisTech, France; Raphaël Blouet, Yacast, France; Jean-Louis Durrieu, Ecole Polytechnique Fédérale de Lausanne, Switzerland

AASP-P4.6: JOINT BAYESIAN REMOVAL OF IMPULSE AND BACKGROUND NOISE

James Murphy, Simon J. Godsill, University of Cambridge, United Kingdom

AASP-P4.7: AN ADAPTIVE NOISE CANCELLER WITH ADAPTIVE DELAY COMPENSATION 265 FOR A DISTANT NOISE SOURCE

Akihiko Sugiyama, Ryoji Miyahara, Masanori Kato, NEC Corporation, Japan

AASP-P4.8: ANALYSIS OF RATE CONSTRAINTS FOR MWF-BASED NOISE REDUCTION IN ACOUSTIC SENSOR NETWORKS

Toby Christian Lawin-Ore, Simon Doclo, University Oldenburg, Germany

AASP-P4.9: A SINGLE-CHANNEL NOISE REDUCTION MVDR FILTER

Jacob Benesty, INRS, Canada; Yiteng (Arden) Huang, WeVoice Inc., United States

AASP-P4.10: ON SINGLE-CHANNEL NOISE REDUCTION IN THE TIME DOMAIN

Jingdong Chen, WeVoice Inc., United States; Jacob Benesty, INRS-EMT / University of Quebec, Canada; Yiteng (Arden) Huang,

WeVoice Inc., United States; Tomas Gaensler, mh acoustics LLC, United States

AASP-P4.11: A VAD-ROBUST MULTICHANNEL WIENER FILTER ALGORITHM FOR NOISE 281 REDUCTION IN HEARING AIDS

Bram Cornelis, Marc Moonen, Jan Wouters, Katholieke Universiteit Leuven, Belgium

\section{AASP-P5: ACOUSTIC SYSTEM MODELLING AND HEARING AIDS}

AASP-P5.1: EFFECT OF FAST AGC ON COCHLEAR IMPLANT SPEECH INTELLIGIBILITY 285

Phyu Khing, Eliathamby Ambikairajah, The University of New South Wales, Australia; Brett Swanson, Cochlear Ltd, Australia

AASP-P5.2: ESTIMATING PRESSURE AND VOLUME VELOCITY IN THE EAR CANAL FOR 289 INSERT HEADPHONES

Marko Hiipakka, Aalto University, Finland

AASP-P5.3: EVALUATION OF TWO SPEECH AND NOISE ESTIMATION METHODS FOR 293 THE ASSESSMENT OF NONLINEAR HEARING AIDS

Nicolas Ellaham, Christian Giguère, Wail Gueaieb, University of Ottawa, Canada

AASP-P5.4: DIRECTIONALITY-BASED SPEECH ENHANCEMENT FOR HEARING AIDS 297

John Woodruff, DeLiang Wang, The Ohio State University, United States

AASP-P5.5: OFFENDING FREQUENCY SUPPRESSION WITH A RESET ALGORITHM TO IMPROVE FEEDBACK CANCELLATION IN DIGITAL HEARING AIDS

Ashutosh Pandey, V. John Mathews, University of Utah, United States

AASP-P5.6: IMPROVING HEAD-RELATED IMPULSE RESPONSE MEASURED IN NOISY 305 ENVIRONMENTS WITH SPATIO-TEMPORAL FREQUENCY ANALYSIS

Takanori Nishino, Mie University, Japan; Kazuya Takeda, Nagoya University, Japan

AASP-P5.7: ESTIMATION OF THE FREQUENCY DEPENDENT REVERBERATION TIME 309 BY MEANS OF WARPED FILTER-BANKS

Heinrich Loellmann, Peter Vary, RWTH Aachen University, Germany 
AASP-P5.8: EQUALIZATION OF MULTICHANNEL ACOUSTIC SYSTEM USING

SUB-SYSTEMS FOR SPEECH DEREVERBERATION

Lei Liao, Andy W. H. Khong, Nanyang Technological University, Singapore

AASP-P5.9: COMPUTING ROOM ACOUSTICS WITH CUDA - 3D FDTD SCHEMES WITH

BOUNDARY LOSSES AND VISCOSITY

Craig Webb, Stefan Bilbao, University of Edinburgh, United Kingdom

AASP-P5.10: CAN ONE HEAR THE SHAPE OF A ROOM: THE 2--D POLYGONAL CASE 321

Ivan Dokmanic, Yue M. Lu, Martin Vetterli, Ecole Polytechnique Fédérale de Lausanne, Switzerland

\section{AASP-P6: AUDIO SIGNAL PROCESSING}

AASP-P6.1: A PERCEPTUALLY TRANSPARENT AUDIO POWER REDUCTION ALGORITHM 325 FOR LOUDSPEAKER POWER MANAGEMENT

Leung Kin Chiu, Nathan Parrish, David V. Anderson, Georgia Institute of Technology, United States

AASP-P6.2: A CONSTRAINED MATCHING PURSUIT APPROACH TO AUDIO DECLIPPING

Amir Adler, Technion, Israel; Valentin Emiya, INRIA Rennes - Bretagne Atlantique, France; Maria G. Jafari, Queen Mary

University of London, United Kingdom; Michael Elad, Technion, Israel; Rémi Gribonval, INRIA Rennes - Bretagne Atlantique,

France; Mark D. Plumbley, Queen Mary University of London, United Kingdom

AASP-P6.3: A FAST PROJECTED GRADIENT OPTIMIZATION METHOD FOR REAL-TIME 333 PERCEPTION-BASED CLIPPING OF AUDIO SIGNALS

Bruno Defraene, Toon van Waterschoot, Moritz Diehl, Marc Moonen, Katholieke Universiteit Leuven, Belgium

AASP-P6.4: AUDIO RECOGNITION IN THE WILD: STATIC AND DYNAMIC 337 CLASSIFICATION ON A REAL-WORLD DATABASE OF ANIMAL VOCALIZATIONS

Felix Weninger, Björn Schuller, Technische Universität München, Germany

AASP-P6.5: BIRD SPECIES RECOGNITION COMBINING ACOUSTIC AND SEQUENCE MODELING

Martin Graciarena, Michelle Delplanche, Elizabeth Shriberg, Andreas Stolcke, SRI International, United States

AASP-P6.6: NOISE ROBUST BIRD SONG DETECTION USING SYLLABLE 345 PATTERN-BASED HIDDEN MARKOV MODELS

Wei Chu, Daniel Blumstein, University of California Los Angeles, United States

AASP-P6.7: IMPROVING ACOUSTIC EVENT DETECTION USING GENERALIZABLE 349 VISUAL FEATURES AND MULTI-MODALITY MODELING

Po-Sen Huang, Xiaodan Zhuang, Mark Hasegawa-Johnson, University of Illinois Urbana-Champaign, United States

AASP-P6.8: AN IMPROVED SCHEME OF AUDIO WATERMARKING BASED ON TURBO 353 CODES AND CHANNEL EFFECT MODELING

Taoufik Majoul, Fathi Raouafi, Meriem Jaidane, Ecole Nationale d'Ingénieurs de Tunis, Tunisia

AASP-P6.9: AUDIO SEGMENTATION OF BROADCAST NEWS: A HIERARCHICAL SYSTEM 357 WITH FEATURE SELECTION FOR THE ALBAYZIN-2010 EVALUATION

Taras Butko, Climent Nadeu, Universitat Politècnica de Catalunya, Spain

AASP-P6.10: DIRECT PROCESSING OF MPEG AUDIO USING COMPANDING AND BFP 361 TECHNIQUES

Christos Vezyrtzis, Aaron Klein, Columbia University, United States; Dan Ellis, Associate Professor/Columbia University, United States; Yannis Tsividis, Columbia University, United States 


\section{AASP-P7: MUSIC SIGNAL PROCESSING III}

AASP-P7.1: CONCURRENT ESTIMATION OF SINGING VOICE FO AND PHONEMES BY

USING SPECTRAL ENVELOPES ESTIMATED FROM POLYPHONIC MUSIC

Hiromasa Fujihara, Masataka Goto, National Institute of Advanced Industrial Science and Technology, Japan

AASP-P7.2: I-DIVERGENCE-BASED DEREVERBERATION METHOD WITH AUXILIARY 369 FUNCTION APPROACH

Naoki Yasuraoka, Kyoto University, Japan; Hirokazu Kameoka, Takuya Yoshioka, NTT Corporation, Japan; Hiroshi G. Okuno, Kyoto University, Japan

AASP-P7.3: AUTOMATIC REAL-TIME ELECTRIC GUITAR AUDIO TRANSCRIPTION

Xander Fiss, Andres Kwasinski, Rochester Institute of Technology, United States

AASP-P7.4: SIC RECEIVER FOR POLYPHONIC PIANO MUSIC

Ana M. Barbancho, Isabel Barbancho, Beatriz Soto, Lorenzo J. Tardon, Universidad de Malaga, Spain

AASP-P7.5: DRUM EXTRACTION FROM POLYPHONIC MUSIC BASED ON A 381 SPECTRO-TEMPORAL MODEL OF PERCUSSIVE SOUNDS

François Rigaud, Institute Telecom / Telecom Paristech, France; Mathieu Lagrange, Institut de Recherche et Coordination Acoustique/Musique / Centre National de la Recherche Scientifique (CNRS), France; Axel Röbel, Geoffroy Peeters, Institut de Recherche et Coordination Acoustique/Musique, France

AASP-P7.6: ESTIMATING NOTE INTENSITIES IN MUSIC RECORDINGS

Sebastian Ewert, University of Bonn, Germany; Meinard Mueller, Saarland University and MPI Informatik, Germany

AASP-P7.7: ADAPTIVE N-NORMALIZATION FOR ENHANCING MUSIC SIMILARITY

Mathieu Lagrange, Institut de Recherche et Coordination Acoustique/Musique / CNRS, France; George Tzanetakis, University of Victoria, Canada

AASP-P7.8: A TREND ESTIMATION ALGORITHM FOR SINGING PITCH DETECTION IN 393 MUSICAL RECORDINGS

Chao-Ling Hsu, National Tsing Hua University, Taiwan; DeLiang Wang, The Ohio State University, United States; Jyh-Shing Roger Jang, National Tsing Hua University, Taiwan

AASP-P7.9: HIDDEN DISCRETE TEMPO MODEL: A TEMPO-AWARE TIMING MODEL FOR 397 AUDIO-TO-SCORE ALIGNMENT

Cyril Joder, Slim Essid, Gaël Richard, Télécom ParisTech, France

AASP-P7.10: ADAPTIVE HARMONIC TIME-FREQUENCY DECOMPOSITION OF AUDIO 401 USING SHIFT-INVARIANT PLCA

Benoît Fuentes, Roland Badeau, Gaël Richard, Télécom ParisTech, France

AASP-P7.11: A HIERARCHICAL GENERATIVE MODEL FOR GENERIC AUDIO DOCUMENT 405 CATEGORIZATION

Zhi Zeng, Shuwu Zhang, Institute of Automation / Chinese Academy of Sciences, China

\section{AASP-P8: ACOUSTIC SIGNAL PROCESSING}

AASP-P8.1: LINEARIZATION ABILITY EVALUATION OF NONLINEAR FILTERS 409 EMPLOYING DYNAMIC DISTORTION MEASUREMENT

Yoshinobu Kajikawa, Kansai University, Japan

AASP-P8.2: A NEW STRUCTURE WITH SPECTRUM-TUNING OF RESIDUAL NOISE FOR 413 ACTIVE NOISE CONTROL

Hua Bao, Issa Panahi, The University of Texas at Dallas, United States 
FEEDBACK ANC

Thomas Schumacher, Hauke Krüger, Marco Jeub, Peter Vary, RWTH Aachen University, Germany; Christophe Beaugeant, Intel

Corporation, France

AASP-P8.4: A METHOD FOR POSTERIOR FREQUENCY-DOMAIN MULTI-CHANNEL 421

RESIDUAL ECHO CANCELING

Satoru Emura, Yoichi Haneda, NTT, Japan

AASP-P8.5: A CLASS OF DOUBLE-TALK DETECTORS BASED ON THE HOLDER 425 INEQUALITY

Constantin Paleologu, University Politehnica of Bucharest, Romania; Jacob Benesty, University of Quebec, Canada; Tomas

Gaensler, mh acoustics LLC, United States; Silviu Ciochina, University Politehnica of Bucharest, Romania

AASP-P8.6: A VARIABLE STEP SIZE EVOLUTIONARY AFFINE PROJECTION ALGORITHM.

Felix Albu, Constantin Paleologu, University Politehnica of Bucharest, Romania; Jacob Benesty, University of Quebec, Canada

AASP-P8.7: ANALYSIS OF ADAPTIVE FEEDBACK AND ECHO CANCELATION ALGORITHMS

IN A GENERAL MULTIPLE-MICROPHONE AND SINGLE-LOUDSPEAKER SYSTEM

Meng Guo, Oticon A/S and Aalborg University, Denmark; Thomas Bo Elmedyb, Oticon A/S, Denmark; Søren Holdt Jensen,

Aalborg University, Denmark; Jesper Jensen, Oticon A/S, Denmark

AASP-P8.8: A CONSTRAINED OPTIMIZATION APPROACH FOR MULTI-ZONE SURROUND

SOUND

Terence Betlehem, Industrial Research Ltd, New Zealand; Paul Teal, Victoria University of Wellington, New Zealand

AASP-P8.9: ROBUST SOUND FIELD REPRODUCTION INTEGRATING MULTI-POINT

SOUND FIELD CONTROL AND WAVE FIELD SYNTHESIS

Noriyoshi Kamado, Hiroshi Saruwatari, Kiyohiro Shikano, Nara Institute of Science and Technology, Japan

AASP-P8.10: A SYSTEM APPROACH TO RESIDUAL ECHO SUPPRESSION IN ROBUST

HANDS-FREE TELECONFERENCING

Jason Wung, Ted S. Wada, Biing-Hwang(Fred) Juang, Georgia Institute of Technology, United States; Bowon Lee, Ton Kalker,

Ronald Schafer, Hewlett-Packard Laboratories, United States

\section{AASP-P9: SOUND REPRODUCTION, SYNTHESIS, AND CLASSIFICATION}

AASP-P9.1: EFFICIENT IMPLEMENTATION OF VIRTUAL 3D SOUND SYNTHESIS BASED 449 ON COMBINING GROUPED PCA AND BMT

Zhixin Wang, Cheung-Fat Chan, City University of Hong Kong, Hong Kong SAR of China

AASP-P9.2: VOCALISTENER2: A SINGING SYNTHESIS SYSTEM ABLE TO MIMIC A 453 USER'S SINGING IN TERMS OF VOICE TIMBRE CHANGES AS WELL AS PITCH AND DYNAMICS

Tomoyasu Nakano, Masataka Goto, National Institute of Advanced Industrial Science and Technology, Japan

AASP-P9.3: STEREO AUDIO CLASSIFICATION FOR AUDIO ENHANCEMENT 457

Aki Härmä, Philips Research, Netherlands

AASP-P9.4: REPRODUCTION OF INDEPENDENT NARROWBAND SOUNDFIELDS IN A 461 MULTIZONE SURROUND SYSTEM AND ITS EXTENSION TO SPEECH SIGNAL SOURCES

Nasim Radmanesh, Ian S. Burnett, RMIT University, Australia

AASP-P9.5: TIME DOMAIN RECONSTRUCTION OF SPATIAL SOUND FIELDS USING 465 COMPRESSED SENSING

Andrew Wabnitz, Nicolas Epain, Andre van Schaik, Craig Jin, University of Sydney, Australia 
AASP-P9.8: AUDIO IDENTIFICATION BASED ON SPECTRAL MODELING OF BARK-BANDS 477 ENERGY AND SYNCHRONIZATION THROUGH ONSET DETECTION

Mathieu Ramona, Geoffroy Peeters, Institut de Recherche et Coordination Acoustique/Musique, France

AASP-P9.9: AUTOMATIC MUSIC TAGGING VIA PARAFAC2 481

Yannis Panagakis, Constantine Kotropoulos, Aristotle University of Thessaloniki, Greece

\section{AASP-P10: AUDIO CODING AND ANALYSIS}

AASP-P10.1: GOSSET LATTICE SPHERICAL VECTOR QUANTIZATION WITH LOW 485 COMPLEXITY

Hauke Krüger, Bernd Geiser, Peter Vary, RWTH Aachen University, Germany; Hai Ting Li, Deming Zhang, Huawei

Technologies Co. Ltd., China

AASP-P10.2: DELAYLESS SOFT-DECISION DECODING OF HIGH-QUALITY AUDIO TRANSMITTED OVER AWGN CHANNELS

Florian Pflug, Tim Fingscheidt, Technische Universität Braunschweig, Germany

AASP-P10.3: EFFICIENT CONTEXT ADAPTIVE ENTROPY CODING FOR REAL-TIME 493 APPLICATIONS

Guillaume Fuchs, Vignesh Subbaraman, Markus Multrus, Fraunhofer Institute for Integrated Circuits (IIS), Germany

AASP-P10.4: EFFICIENT TRANSFORM CODING OF TWO-CHANNEL AUDIO SIGNALS BY 497 MEANS OF COMPLEX-VALUED STEREO PREDICTION

Christian R. Helmrich, Fraunhofer Institute for Integrated Circuits (IIS), Germany; Pontus Carlsson, Dolby Sweden AB, Sweden; Sascha Disch, Bernd Edler, Johannes Hilpert, Matthias Neusinger, Fraunhofer Institute for Integrated Circuits (IIS), Germany; Heiko Purnhagen, Dolby Sweden AB, Sweden; Nikolaus Rettelbach, Julien Robilliard, Fraunhofer Institute for Integrated Circuits (IIS), Germany; Lars Villemoes, Dolby Sweden AB, Sweden

AASP-P10.5: ENHANCED CODING OF HIGH-FREQUENCY TONAL COMPONENTS IN 501 MPEG-D USAC THROUGH JOINT APPLICATION OF ESBR AND SINUSOIDAL MODELING

Tomasz Zernicki, Telcordia Poland Sp. z o. o., Poland; Maciej Bartkowiak, Marek Domanski, Poznan University of Technology, Poland

AASP-P10.6: ENHANCED LONG-TERM PREDICTOR FOR UNIFIED SPEECH AND AUDIO 505 CODING

Jeongook Song, Hyen-O Oh, Hong-Goo Kang, Yonsei University, Republic of Korea

AASP-P10.7: IMPROVED PHASE PARAMETER ANALYSIS AND SYNTHESIS FOR 509 PARAMETRIC STEREO AUDIO CODING

Dong-il Hyun, Yonsei University, Republic of Korea; Jeongil Seo, Electronics and Telecommunications Research Institute, Republic of Korea; Young-cheol Park, Dae Hee Youn, Yonsei University, Republic of Korea

AASP-P10.8: AUDIO SIGNAL REPRESENTATIONS FOR FACTORIZATION IN THE SPARSE 513 DOMAIN

Manuel Moussallam, Institut Telecom / Telecom ParisTech / CNRS/LTCI, France; Laurent Daudet, Institut Langevin - ESPCI ParisTech - UMR7587, France; Gaël Richard, Institut Telecom / Telecom ParisTech / CNRS/LTCI, France

AASP-P10.9: DETECTION OF SINUSOIDAL SIGNALS IN NOISE BY PROBABILISTIC 517 MODELLING OF THE SPECTRAL MAGNITUDE SHAPE AND PHASE CONTINUITY Peter Jancovic, Munevver Kokuer, University of Birmingham, United Kingdom

AASP-P10.10: SPECTRAL-ENVELOPE AND GROUP-DELAY MODELS FOR TRANSIENT 521 SIGNALS --- APPLICATIONS TO CASTANETS AND STOP CONSONANTS

Ravi R. Shenoy, Chandra Sekhar Seelamantula, Indian Institute of Science, India 


\section{BISP-L1: BIOSIGNAL ESTIMATION AND CLASSIFICATION}

BISP-L1.1: SEQUENTIAL MONTE CARLO METHOD FOR PARAMETER ESTIMATION IN 525 DIFFUSION MODELS OF AFFINITY-BASED BIOSENSORS

Manohar Shamaiah, Xiaohu Shen, Haris Vikalo, The University of Texas at Austin, United States

BISP-L1.2: ITERATIVE ESTIMATION OF STRUCTURES OF MULTIPLE RNA 529 HOMOLOGS: TURBOFOLD

Gaurav Sharma, Arif Harmanci, University of Rochester, United States; David Mathews, University of Rochester Medical Center, United States

BISP-L1.3: SPARSE COMMON SPATIAL PATTERNS IN BRAIN COMPUTER INTERFACE 533 APPLICATIONS

Fikri Goksu, N. Firat Ince, Ahmed H. Tewfik, University of Minnesota, United States

BISP-L1.4: P AND T WAVE DELINEATION AND WAVEFORM ESTIMATION IN ECG 537 SIGNALS USING A BLOCK GIBBS SAMPLER

Chao Lin, University of Toulouse, France; Georg Kail, Vienna University of Technology, Austria; Jean-Yves Tourneret, Corinne Mailhes, University of Toulouse, France; Franz Hlawatsch, Vienna University of Technology, Austria

BISP-L1.5: ODOR STIMULUS INFERENCE BASED ON NEURAL SPIKE SIGNAL IN RATS 541 Kyung-Jin You, Soongsil University, Republic of Korea; Hyun Joo Lee, Yiran Lang, Changkyun Im, Chin Su Koh, Hyung-Cheul Shin, Hallym University, Republic of Korea; Hyun-Chool Shin, Soongsil University, Republic of Korea

BISP-L1.6: A FAST SOLUTION TO ROBUST MINIMUM VARIANCE BEAMFORMER AND 545 APPLICATION TO SIMULTANEOUS MEG AND LOCAL FIELD POTENTIAL

Hamid Mohseni, Morten Kringelbach, Mark Woolrich, Penny Probert Smith, Tipu Aziz, University of Oxford, United Kingdom

\section{BISP-L2: MEDICAL IMAGING II}

BISP-L2.1: CALIBRATION IN CIRCULAR ULTRASOUND TOMOGRAPHY DEVICES 549

Reza Parhizkar, Amin Karbasi, Martin Vetterli, Ecole Polytechnique Fédérale de Lausanne, Switzerland

BISP-L2.2: COMBINED COMPRESSED SENSING AND PARALLEL MRI COMPARED FOR 553 UNIFORM AND RANDOM CARTESIAN UNDERSAMPLING OF K-SPACE

Daniel S. Weller, Massachusetts Institute of Technology, United States; Jonathan R. Polimeni, Massachusetts General Hospital, United States; Leo Grady, Siemens Corporate Research, United States; Lawrence L. Wald, Massachusetts General Hospital, United States; Elfar Adalsteinsson, Vivek K. Goyal, Massachusetts Institute of Technology, United States

BISP-L2.3: DYNAMICS OF TONGUE GESTURES EXTRACTED AUTOMATICALLY FROM 557 ULTRASOUND

Jeff Berry, Ian Fasel, University of Arizona, United States

BISP-L2.4: 3D MEDICAL IMAGE CODING WITH OPTIMAL CHANNEL PROTECTION FOR 561 WIRELESS TRANSMISSION

Victor Sanchez, Panos Nasiopoulos, University of British Columbia, Canada

BISP-L2.5: REAL-TIME CONJUGATE GRADIENTS FOR ONLINE FMRI CLASSIFICATION 565

Hao Xu, Yongxin Xi, Ray Lee, Peter Ramadge, Princeton University, United States

BISP-L2.6: SPARSITY-BASED SINOGRAM DENOISING FOR LOW-DOSE COMPUTED 569 TOMOGRAPHY

Joseph Shtok, Michael Elad, Michael Zibulevsky, Technion / Israel Institute of Technology, Israel 


\section{BISP-L3: BIOSIGNAL PROCESSING}

BISP-L3.1: ESTIMATION OF FUNDAMENTAL FREQUENCY FROM SURFACE

ELECTROMYOGRAPHIC DATA: EMG-TO-FO

Keigo Nakamura, Matthias Janke, Michael Wand, Tanja Schultz, Karlsruhe Institute of Technology, Germany

BISP-L3.2: STATIONARY COMMON SPATIAL PATTERNS: TOWARDS ROBUST 577

CLASSIFICATION OF NON-STATIONARY EEG SIGNALS

Wojciech Wojcikiewicz, Carmen Vidaurre, Technical University of Berlin, Germany; Motoaki Kawanabe, Fraunhofer Institute FIRST, Germany

BISP-L3.3: COMPRESSION OF QRS COMPLEXES USING HERMITE EXPANSION

Aliaksei Sandryhaila, Jelena Kovacevic, Carnegie Mellon University, United States; Markus Püschel, ETH Zürich, Switzerland

BISP-L3.4: CLASSIFICATION BY WEIGHTING FOR SPATIO-FREQUENCY COMPONENTS 585 OF EEG SIGNAL DURING MOTOR IMAGERY

Hiroshi Higashi, Toshihisa Tanaka, Tokyo University of Agriculture and Technology, Japan

BISP-L3.5: MULTICHANNEL EEG ANALYSIS BASED ON MULTI-SCALE 589 MULTI-INFORMATION

Ying Liu, Selin Aviyente, Michigan State University, United States

BISP-L3.6: INSTANTANEOUS PHASE TRACKING OF OSCILLATORY SIGNALS USING EMD 593 AND RAO-BLACKWELLISED PARTICLE FILTERING

Delaram Jarchi, Bahador Makkiabadi, Saeid Sanei, University of Surrey, United Kingdom

\section{BISP-P1: BIOINFORMATICS AND BIOSIGNALS}

BISP-P1.1: ITERATIVE PCA FOR POPULATION STRUCTURE ANALYSIS

597

Tulaya Limpiti, King Mongkut's Institute of Technology Ladkrabang, Thailand; Apichart Intarapanich, National Electronics and Computer Technology Center, Thailand; Anunchai Assawamakin, Pongsakorn Wangkumhang, Sissades Tongsima, National Center for Genetic Engineering and Biotechnology, Thailand

BISP-P1.2: A MUTUAL INFORMATION BASED APPROACH FOR EVALUATING THE 601 QUALITY OF CLUSTERING

Shaikh Anowarul Fattah, Chia-Chun Lin, Sun-Yuan Kung, Princeton University, United States

BISP-P1.3: A FULLY AUTOMATED 2-DE GEL IMAGE ANALYSIS PIPELINE FOR HIGH 605 THROUGHPUT PROTEOMICS

Tsakanikas Panagiotis, Elias Manolakos, University of Athens, Greece

BISP-P1.4: WAVELET FOOTPRINTS FOR DETECTION AND SORTING OF 609 EXTRACELLULAR NEURAL ACTION POTENTIALS

Ki Yong Kwon, Karim Oweiss, Michigan State University, United States

BISP-P1.5: MULTIPLE PITCH IDENTIFICATION USING COCHLEAR-LIKE FREQUENCY 613 CAPTURE AND HARMONIC GROUPING

Kumaresan Ramdas, Vijay Kumar Peddinti, University of Rhode Island, United States; Cariani Peter, Harvard Medical School, United States

BISP-P1.6: DECONVOLUTION OF NEURONAL SIGNAL FROM HEMODYNAMIC 617 RESPONSE

Martin Havlicek, Jiri Jan, Brno University of Technology, Czech Republic; Milan Brazdil, St. Anne's University Hospital, Czech Republic; Vince D, Calhoun, The Mind Research Network, United States

BISP-P1.7: DETECTION OF PEPTIDE ION PEAKS IN MASS SPECTRA BY USING 621 WEIGHTED AUTO-CORRELATION

Kenji Watanabe, Takumi Kobayashi, Katsuyuki Koike, Tetsuya Higuchi, Tohru Natsume, Nobuyuki Otsu, National Institute of Advanced Industrial Science and Technology, Japan 


\section{BISP-P2: PROCESSING OF PHYSIOLOGICAL SIGNALS I}

BISP-P2.1: MULTI-CHANNEL EEG COMPRESSION BASED ON MATRIX AND TENSOR 629 DECOMPOSITIONS

Justin Dauwels, National Technological University, Singapore; Srinivasan Kannan, Reddy Ramasubba, IIT Madras, Singapore; Andrzej Cichocki, RIKEN Brain Science Institute, Japan

BISP-P2.2: FROM COMPRESSIVE TO ADAPTIVE SAMPLING OF NEURAL AND ECG 633 RECORDINGS

Alexander Singh Alvarado, Jose C. Principe, University of Florida, United States

BISP-P2.3: JOINT MODELING OF OBSERVED INTER-ARRIVAL TIMES AND WAVEFORM 637 DATA WITH MULTIPLE HIDDEN STATES FOR NEURAL SPIKE-SORTING

Brett Matthews, Mark Clements, Georgia Institute of Technology, United States

BISP-P2.4: TIME-FREQUENCY ANALYSIS COMPENSATING MISSING DATA FOR ATRIAL 641 FIBRILLATION ECG ASSESSMENT

Sandun Kodituwakku, Rodney A. Kennedy, Thushara D. Abhayapala, Australian National University, Australia

BISP-P2.5: A COLLABORATIVE FILTERING APPROACH FOR QUASI-BRAIN-DEATH EEG 645 ANALYSIS

Yili Xia, Ling Li, Imperial College London, United Kingdom; Jianting Cao, Saitama Institute of Technology, Japan; Martin Golz, University of Applied Sciences Schmalkalden, Germany; Danilo P. Mandic, Imperial College London, United Kingdom

BISP-P2.6: NEONATAL SEIZURE DETECTION USING BLIND MULTICHANNEL 649 INFORMATION FUSION

Huaying Li, Aleksandar Jeremic, McMaster University, Canada

BISP-P2.7: SEPARATING SOURCES FROM SEQUENTIALLY ACQUIRED MIXTURES OF 653 HEART SIGNALS

Fabio L. Hedayioglu, University of Porto, Portugal; Maria G. Jafari, Queen Mary University of London, United Kingdom; Sandra S. Mattos, Royal Portuguese Hospital, Brazil; Mark D. Plumbley, Queen Mary University of London, United Kingdom; Miguel T. Coimbra, University of Porto, Portugal

BISP-P2.8: EFFECT OF THE VISUAL SIGNAL STRUCTURE ON STEADY-STATE VISUAL 657 EVOKED POTENTIALS DETECTION

Hubert Cecotti, Bertrand Rivet, GIPSA-LAB / CNRS UMR5216, France

BISP-P2.9: AN ADAPTIVE APPROACH TO ABNORMAL HEART SOUND SEGMENTATION 661

Dinesh Kumar, Paulo Carvalho, University of Coimbra, Portugal; Manuel Antunes, Centre of Cardio-thoracic Surgery of the University Hospital of Coimbra, Portugal; Rui Pedro Paiva, Jorge Henriques, University of Coimbra, Portugal

BISP-P2.10: EPILEPTIC SEIZURE PREDICTION USING THE SPATIOTEMPORAL 665 CORRELATION STRUCTURE OF INTRACRANIAL EEG

James Williamson, Daniel Bliss, David Browne, MIT Lincoln Laboratory, United States

\section{BISP-P3: SIGNAL PROCESSING IN HEALTH MONITORING}

BISP-P3.1: SIGNAL-BASED SEGMENTATION OF HUMAN LOCOMOTION USING 669 EMBEDDED SENSOR NETWORK

Maud Pasquier, Bernard Espiau, INRIA, France; Christine Azevedo-Coste, LIRMM, France 

COEFFICIENTS: IMPLICATIONS FOR OBSTRUCTIVE SLEEP APNEA DIAGNOSIS

Hisham Alshaer, University of Toronto, Canada; Martha Garcia, Universidad Iberoamericana, Mexico; Hossein Radfar, University of Toronto, Canada; Geoffrey R. Fernie, Toronto Rehabilitation Institute, Canada; T. Douglas Bradley, University of Toronto, Canada

BISP-P3.5: AUTOMATIC DIAGNOSIS OF ADHD BASED ON NONLINEAR ANALYSIS OF 685 ACTIMETRY REGISTRIES

Diego Martín, Pablo Casaseca, University of Valladolid, Spain; Susana Alberola, José Antonio López, Francisco Carlos Ruiz, Jesús María Andrés, José Ramón Garmendia, SACYL, Spain; Julio Ardura, University of Valladolid, Spain

BISP-P3.6: A GRAPH BASED METHOD FOR TIMED UP \& GO TEST QUALIFICATION 689 USING INERTIAL SENSORS

Pierre Jallon, Benjamin Dupré, Michel Antonakios, CEA/LETI, France

BISP-P3.7: DISCRIMINATION BETWEEN HEALTHY SUBJECTS AND PATIENTS WITH 693 PULMONARY EMPHYSEMA BY DETECTION OF ABNORMAL RESPIRATION

Masaru Yamashita, Shoichi Matsunaga, Sueharu Miyahara, Nagasaki University, Japan

BISP-P3.8: ANALYSIS OF HUMAN FOOTSTEPS UTILIZING MULTI-AXIAL SEISMIC 697 FUSION

Sean Schumer, United States Army, United States

BISP-P3.9: PERFORMANCE ANALYSIS OF HURST'S EXPONENT ESTIMATORS IN 701 HIGHLY IMMATURE BREATHING PATTERNS OF PRETERM INFANTS

Xavier Navarro, Alain Beuchée, Fabienne Porée, Guy Carrault, Université Rennes 1, France

\section{BISP-P4: MEDICAL IMAGING I}

BISP-P4.1: CONTOUR-BASED HIDDEN MARKOV MODEL TO SEGMENT 2D 705 ULTRASOUND IMAGES

Xiaoning Qian, USF, United States; Byung-Jun Yoon, Texas A\&M University, United States

BISP-P4.2: CHARACTERIZATION OF THREE-DIMENSIONAL DATA WITH MULTIDIMENSIONAL DEFORMABLE MODELS BASED ON B-SPLINES IN THE FOURIER DOMAIN

Rafael Berenguer-Vidal, Rafael Verdú-Monedero, Juan Morales-Sánchez, J. Larrey-Ruiz, María Consuelo Bastida-Jumilla, Universidad Politécnica de Cartagena, Spain

BISP-P4.3: SEGMENTATION OF THE CAROTID ARTERY IN ULTRASOUND IMAGES 713 USING FREQUENCY-DESIGNED B-SPLINE ACTIVE CONTOUR José Luis Izquierdo-Zaragoza, María Consuelo Bastida-Jumilla, Rafael Verdú-Monedero, Juan Morales-Sánchez, Rafael Berenguer-Vidal, Universidad Politécnica de Cartagena, Spain 
ACTIVATION

Victor Solo, Ben Cassidy, University of New South Wales, Australia; Christopher Long, GlaxoSmithKline, United Kingdom;

Caroline Rae, Neuroscience Research Australia, Australia

BISP-P4.7: LABELING SKIN TISSUES IN ULTRASOUND IMAGES USING A GENERALIZED

RAYLEIGH MIXTURE MODEL

Marcelo A. Pereyra, Nicolas Dobigeon, Hadj Batatia, Jean-Yves Tourneret, University of Toulouse, France

BISP-P4.8: SMOOTH INTENSITY MAPS FOR IMRT

Mónica Hernández Giménez, Juan Manuel Artacho Terrer, Xavier Mellado Esteban, Santiago Cruz Llanas, Aragón Institute for

Engineering Research, Spain

BISP-P4.9: JOINT REDUCE OF METAL AND BEAM HARDENING ARTIFACTS USING 737

MULTI-ENERGY MAP APPROACH IN X-RAY COMPUTED TOMOGRAPHY

Yuling Zheng, Caifang Cai, Thomas Rodet, Laboratoire des Signaux et Systèmes (CNRS-SUPELEC-Univ. Paris-Sud), France

BISP-P4.10: DEREVERBERATION OF ULTRASOUND ECHO DATA IN VASCULAR IMAGING 741 APPLICATIONS

Emad Ebbini, Yayun Wan, Dalong Liu, University of Minnesota, United States

\section{BISP-P5: PROCESSING OF PHYSIOLOGICAL SIGNALS II}

BISP-P5.1: GRAPHICAL MODELS FOR LOCALIZATION OF THE SEIZURE FOCUS FROM 745 INTERICTAL INTRACRANIAL EEG

Justin Dauwels, National Technological University, Singapore; Emad Eskandar, Andy Cole, Dan Hoch, Rodrigo Zepeda, MGH and Harvard Medical School, United States; Sydney S. Cash, Massachusetts General Hospital / Harvard Medical School, United States

BISP-P5.2: POWER ESTIMATION SCHEME FOR LOW POWER ORIENTED BIOMEDICAL 749 SOC EXTENDED TO VERY DEEP SUBMICRON TECHNOLOGY

Hong-Hui Chen, Tung-Chien Chen, Cheng-Yi Chiang, Liang-Gee Chen, National Taiwan University, Taiwan

BISP-P5.3: QUANTIFYING THE FUNCTIONAL IMPORTANCE OF NEURONAL 753 ASSEMBLIES IN THE BRAIN USING LAPLACIAN HUCKEL GRAPH ENERGY

Marcos Bolanos, Selin Aviyente, Michigan State University, United States

BISP-P5.4: ANALYSIS OF PHONE CONFUSION IN EMG-BASED SPEECH RECOGNITION.

Michael Wand, Tanja Schultz, Karlsruhe Institute of Technology, Germany

BISP-P5.5: COMPRESSED SENSING BASED METHOD FOR ECG COMPRESSION

Luisa Polania, Rafael Carrillo, University of Delaware, United States; Manuel Blanco-Velasco, Universidad de Alcala, Spain; Kenneth Barner, University of Delaware, United States

BISP-P5.6: IMPROVED TRANSIENT OSCILLATION DETECTION WITH 765 MULTIWAVELETS

Melani Plett, Seattle Pacific University, United States

BISP-P5.7: ESTIMATION OF CORTICAL CONNECTIVITY FROM E/MEG USING 769 NONLINEAR STATE-SPACE MODELS

Bing Leung Cheung, Barry Van Veen, University of Wisconsin Madison, United States

BISP-P5.8: TOPOGRAPHIC PHASE MAPS USING ITERATIVE INDEPENDENT

COMPONENT ANALYSIS

Darshan Iyer, NEUROMetrix Inc., United States; George Zouridakis, Biomedical Imaging Lab, United States

BISP-P5.9: MESSAGE-PASSING FOR BASE-CALLING IN SEQUENCING-BY-SYNTHESIS 777 SYSTEMS

Sang Hyun Lee, Manohar Shamaiah, Haris Vikalo, The University of Texas at Austin, United States 
BISP-P5.10: SVM FEATURE SELECTION FOR MULTIDIMENSIONAL EEG DATA 781

Nisrine Jrad, Ronald Phlypo, Marco Congedo, Institut national polytechnique de Grenoble, France

BISP-P5.11: ROBUST REPRESENTATIONS OF CORTICAL SPEECH AND LANGUAGE 785 INFORMATION

Janet Baker, Saras Institute, United States; Alexander Chan, Massachusetts General Hospital, United States; Ksenija Marinkovic, Eric Halgren, University of California San Diego, United States; Sydney Cash, Massachusetts General Hospital, United States

\section{IVMSP-L1: IMAGE CODING}

IVMSP-L1.1: IMAGE PREDICTION BASED ON NON-NEGATIVE MATRIX FACTORIZATION. 789

Mehmet Turkan, Christine Guillemot, INRIA/IRISA, France

IVMSP-L1.2: IMAGE COMPRESSION USING THE ITERATION-TUNED AND ALIGNED 793 DICTIONARY

Joaquin Zepeda, Christine Guillemot, Ewa Kijak, INRIA, France

IVMSP-L1.3: LOW-COMPLEXITY PREDICTIVE LOSSY COMPRESSION OF 797 HYPERSPECTRAL AND ULTRASPECTRAL IMAGES Andrea Abrardo, Mauro Barni, University of Siena, Italy; Enrico Magli, Politecnico di Torino, Italy

IVMSP-L1.4: RATE-DISTORTION IMPROVEMENT OF DIRECTIONAL WAVELETS BY 801 MEGABLOCKING

Arian Maleki, Standord University, United States; Boshra Rajaei, Hamid-Reza Pourreza, Ferdowsi University of Mashhad, Iran

IVMSP-L1.5: INTRA-FRAME PREDICTION WITH LAPPED TRANSFORMS FOR IMAGE 805 CODING

Rafael Galvão de Oliveira, Béatrice Pesquet-Popescu, Télécom ParisTech, France

IVMSP-L1.6: OPTIMAL STRUCTURE OF MEMORY MODELS FOR LOSSLESS 809 COMPRESSION OF BINARY IMAGE CONTOURS

Ioan Tabus, Septimia Sarbu, Tampere University of Technology, Finland

\section{IVMSP-L2: VIDEO CODING II}

IVMSP-L2.1: IMPROVED P-DOMAIN RATE CONTROL WITH ACCURATE HEADER SIZE . 813 ESTIMATION

Fan Zhang, Eckehard Steinbach, Technische Universitaet Muenchen, Germany

IVMSP-L2.2: INTEGRATING A SPATIAL JUST NOTICEABLE DISTORTION MODEL IN THE 817 UNDER DEVELOPMENT HEVC CODEC

Matteo Naccari, Fernando Pereira, Instituto de Telecomunicações / University of Porto, Portugal

IVMSP-L2.3: FOR/SOR VIDEO CODING WITH SUPER MACROBLOCK AND 821 INTER-FRAME STRIPE PREDICTION

Je-won Kang, Seung-Hwan Kim, C. -C. Jay Kuo, University of Southern California, United States

IVMSP-L2.4: A SPECTRAL APPROACH TO RECURSIVE END-TO-END DISTORTION 825 ESTIMATION FOR SUB-PIXEL MOTION-COMPENSATED VIDEO CODING Jingning Han, University of California Santa Barbara, United States; Vinay Melkote, Dolby Laboratories Inc., United States; Kenneth Rose, University of California Santa Barbara, United States

IVMSP-L2.5: TEMPORALLY COHERENT LUMINANCE-TO-LUMA MAPPING FOR HIGH 829 DYNAMIC RANGE VIDEO CODING WITH H.264/AVC

Jens-Uwe Garbas, Herbert Thoma, Fraunhofer Institute for Integrated Circuits (IIS), Germany 


\section{IVMSP-L3: STEREOSCOPIC AND 3-D CODING}

IVMSP-L3.1: DENSE DISPARITY ESTIMATION FROM LINEAR MEASUREMENTS

Vijayaraghavan Thirumalai, Pascal Frossard, Ecole Polytechnique Fédérale de Lausanne, Switzerland

IVMSP-L3.2: STRETCHING, COMPRESSION AND SHEARING DISPARITY COMPENSATED PREDICTION TECHNIQUES FOR STEREO AND MULTIVIEW VIDEO CODING

Ka-Man Wong, Lai-Man Po, City University of Hong Kong, Hong Kong SAR of China; Kwok-Wai Cheung, Chu Hai College of Higher Education, Hong Kong SAR of China; Ka-Ho Ng, Xuyuan Xu, City University of Hong Kong, Hong Kong SAR of China

IVMSP-L3.3: EXTENDED INTER-VIEW DIRECT MODE FOR MULTIVIEW VIDEO 845 CODING

Jacek Konieczny, Marek Domanski, Poznan University of Technology, Poland

IVMSP-L3.4: AUTO-REGRESSIVE MODEL BASED ERROR CONCEALMENT SCHEME FOR STEREOSCOPIC VIDEO CODING

Xinguang Xiang, Debin Zhao, Harbin Institute of Technology, China; Siwei Ma, Wen Gao, Peking University, China

IVMSP-L3.5: PROXIMAL SPLITTING METHODS FOR DEPTH ESTIMATION. 853

Mireille El Gheche, Jean-Christophe Pesquet, Université Paris-Est Marne la Vallée, France; Joumana Farah, Holy-Spirit University of Kaslik, Lebanon; Mounir Kaaniche, Béatrice Pesquet-Popescu, Télécom ParisTech, France

IVMSP-L3.6: RATE DISTORSION ANALYSIS IN A DISPARITY COMPENSATED SCHEME 857

Valentina Davidoiu, Thomas Maugey, Béatrice Pesquet-Popescu, Télécom ParisTech, France; Pascal Frossard, Ecole

Polytechnique Fédérale de Lausanne, Switzerland

\section{IVMSP-L4: IMAGE AND VIDEO INDEXING AND RETRIEVAL}

IVMSP-L4.1: SEARCHING IN ONE BILLION VECTORS: RE-RANK WITH SOURCE CODING

Hervé Jégou, INRIA, France; Romain Tavenard, University of Rennes 1, France; Matthijs Douze, INRIA, France; Laurent Amsaleg, CNRS, France

IVMSP-L4.2: MULTIVARIATE TEXTURE RETRIEVAL USING THE SIRV 865 REPRESENTATION AND THE GEODESIC DISTANCE

Lionel Bombrun, Nour-Eddine Lasmar, Yannick Berthoumieu, IMS, France; Geert Verdoolaege, Ghent University, Belgium

IVMSP-L4.3: TOPIC-SENSITIVE INTERACTIVE IMAGE OBJECT RETRIEVAL WITH 869 NOISE-PROOF RELEVANCE FEEDBACK

Jen-Hao Hsiao, IBM Research Collaboratory, Taiwan, Taiwan; Henry Chang, IBM T.J. Watson Research Center, United States

IVMSP-L4.4: A GENERAL FRAMEWORK FOR ROBUST HOSVD-BASED INDEXING AND 873 RETRIEVAL WITH HIGH-ORDER TENSOR DATA

Qun Li, Xiangqiong Shi, Dan Schonfeld, University of Illinois Chicago, United States

IVMSP-L4.5: EVENT CLASSIFICATION FOR PERSONAL PHOTO COLLECTIONS . 877

Feng Tang, Daniel R. Tretter, Chris Willis, Hewlett-Packard Laboratories, United States

IVMSP-L4.6: FAST IDENTIFICATION OF JPEG 2000 IMAGES FOR DIGITAL CINEMA 881 PROFILES

Osamu Watanabe, Takushoku University, Japan; Takahiro Fukuhara, Sony Corp., Japan; Hitoshi Kiya, Tokyo Metropolitan University, Japan

\section{IVMSP-L5: STEREO AND 3-D PROCESSING}

IVMSP-L5.1: SPATIO-TEMPORAL CONSISTENCY IN VIDEO DISPARITY ESTIMATION. 
IVMSP-L5.3: JOINT DISPARITY AND OPTICAL FLOW BY CORRESPONDENCE 893 GROWING

Jan Cech, Radu Horaud, INRIA, France

IVMSP-L5.4: SEMI-AUTOMATIC 2D TO 3D IMAGE CONVERSION USING A HYBRID 897 RANDOM WALKS AND GRAPH CUTS BASED APPROACH

Raymond Phan, Richard Rzeszutek, Dimitrios Androutsos, Ryerson University, Canada

IVMSP-L5.5: ACCURATE NON-ITERATIVE DEPTH LAYER EXTRACTION ALGORITHM 901 FOR IMAGE BASED RENDERING

James Pearson, Pier-Luigi Dragotti, Mike Brookes, Imperial College London, United Kingdom

IVMSP-L5.6: SPATIALLY CONSISTENT VIEW SYNTHESIS WITH COORDINATE 905 ALIGNMENT

Lam Tran, Ramsin Khoshabeh, Ankit Kumar Jain, University of California San Diego, United States; Christopher Pal, Ecole Polytechnique in Montreal, Canada; Truong Q. Nguyen, University of California San Diego, United States

\section{IVMSP-L6: VIDEO ANALYSIS AND PROCESSING}

IVMSP-L6.1: EFFECTIVE MULTI-RESOLUTION BACKGROUND SUBTRACTION

Lingfeng Wang, Chunhong Pan, Institute of Automation / Chinese Academy of Sciences, China

IVMSP-L6.2: OCCLUSION BOUNDARY DETECTION USING AN ONLINE LEARNING 913 FRAMEWORK

Natan Jacobson, Yoav Freund, Truong Q. Nguyen, University of California San Diego, United States

IVMSP-L6.3: DISSOLVE DETECTION IN ABSTRACT VIDEO CONTENTS. 917

Bogdan Ionescu, Constantin Vertan, University Politehnica of Bucharest, Romania; Patrick Lambert, University of Savoie,

France

IVMSP-L6.4: A PROBABILISTIC PIXEL-BASED APPROACH TO DETECT HUMANS IN 921 VIDEO STREAMS

Sébastien Piérard, Antoine Lejeune, Marc Van Droogenbroeck, University of Liège, Belgium

IVMSP-L6.5: DETECTING MOVING OBJECTS FROM DYNAMIC BACKGROUND WITH 925 SHADOW REMOVAL

Shih-Chieh Wang, Te-Feng Su, Shang-Hong Lai, National Tsing Hua University, Taiwan

IVMSP-L6.6: A REGION BASED APPROACH TO BACKGROUND MODELING IN A 929 WAVELET MULTI-RESOLUTION FRAMEWORK

Ainhoa Mendizabal, Luis Salgado, Universidad Politécnica de Madrid, Spain

\section{IVMSP-L7: IMAGE RESTORATION AND ENHANCEMENT}

IVMSP-L7.1: ON COOPERATIVE IMAGE DENOISING.

Maciej Niedzwiecki, Szymon Gackowski, Gdansk University of Technology, Poland

IVMSP-L7.2: A NEW VARIATIONAL METHOD FOR PRESERVING POINT-LIKE AND

Daniele Graziani, Laure Blanc-Feraud, CNRS/INRIA, France; Gilles Aubert, Université de Nice Sophia-Antipolis, France 
Camille Couprie, Hugues Talbot, Jean-Christophe Pesquet, Laurent Najman, Université Paris Est, France; Leo Grady, Siemens Corporate Research, United States

IVMSP-L7.5: BOUNDED GRADIENT PROJECTION METHODS FOR SPARSE SIGNAL 949 RECOVERY

James Hernandez, University of California Merced, United States; Zachary Harmany, Duke University, United States; Daniel Thompson, Roummel Marcia, University of California Merced, United States

IVMSP-L7.6: MOTION VECTOR RECOVERY WITH GAUSSIAN PROCESS REGRESSION 953

Hadi Asheri, Abdolkhalegh Bayati, Hamid R. Rabiee, Mohammad H. Rohban, Sharif University of Technology, Iran

\section{IVMSP-L8: IMAGE FEATURE EXTRACTION AND ANALYSIS}

IVMSP-L8.1: VARIATIONAL METHODS FOR SPECTRAL UNMIXING OF 957 HYPERSPECTRAL IMAGES

Olivier Eches, Nicolas Dobigeon, Jean-Yves Tourneret, University of Toulouse, France; Hichem Snoussi, University of Technology of Troyes, France

IVMSP-L8.2: IMAGE NOISE-INFORMATIVE MAP FOR NOISE STANDARD DEVIATION 961 ESTIMATION

Mykhail Uss, Benoit Vozel, University of Rennes 1 / TSI2M, France; Vladimir Lukin, National Aerospace University / Department of Receivers, Transmitters and Signal Processing, Ukraine; Igor Baryshev, National Aerospace University / Department of Radioelectronic Systems and Complexes, Ukraine; Kacem Chehdi, University of Rennes 1 / TSI2M, France

IVMSP-L8.3: BEYOND BAG OF WORDS: COMBINING GENERATIVE AND 965 DISCRIMINATIVE MODELS FOR NATURAL SCENE CATEGORIZATION

Zhen Li, Kim-Hui Yap, Nanyang Technological University, Singapore; Xiao-Ming Chen, Sun Yat-Sen University, China

IVMSP-L8.4: WHOLE-PAINTING CANVAS ANALYSIS USING HIGH- AND LOW-LEVEL 969 FEATURES

Don Johnson, Rice University, United States; Robert Erdmann, University of Arizona, United States; C. Richard Johnson Jr., Cornell University, United States

IVMSP-L8.5: LINEAR MANIFOLD APPROXIMATION BASED ON DIFFERENCES OF 973 TANGENTS

Sofia Karygianni, Pascal Frossard, Ecole Polytechnique Fédérale de Lausanne, Switzerland

IVMSP-L8.6: APPROXIMATION OF PATTERN TRANSFORMATION MANIFOLDS WITH 977 PARAMETRIC DICTIONARIES

Elif Vural, Pascal Frossard, Ecole Polytechnique Fédérale de Lausanne, Switzerland

\section{IVMSP-L9: INTERPOLATION AND SUPER-RESOLUTION}

IVMSP-L9.1: ON THE IMPROVEMENT OF IMAGE REGISTRATION FOR HIGH 981 ACCURACY SUPER-RESOLUTION

Michalis Vrigkas, Christophoros Nikou, Lisimachos P. Kondi, University of Ioannina, Greece

IVMSP-L9.2: A REVISIT TO MRF-BASED DEPTH MAP SUPER-RESOLUTION AND 985 ENHANCEMENT

Jiangbo Lu, Dongbo Min, Advanced Digital Sciences Center, Singapore; Ramanpreet Singh Pahwa, Minh N. Do, University of Illinois Urbana-Champaign, United States

IVMSP-L9.3: AN EVOLUTIONARY GAME-THEORETIC APPROACH FOR IMAGE 989 INTERPOLATION

Yan Chen, Yang Gao, K. J. Ray Liu, University of Maryland College Park, United States 
IVMSP-L9.4: EDGE ORIENTED DIRECTIONAL COLOR FILTER ARRAY INTERPOLATION 993

Ibrahim Pekkucuksen, Yucel Altunbasak, Georgia Institute of Technology, United States

IVMSP-L9.5: DIRECTIONAL COLOR FILTER ARRAY INTERPOLATION BASED ON 997 MULTISCALE COLOR GRADIENTS

Ibrahim Pekkucuksen, Yucel Altunbasak, Georgia Institute of Technology, United States

IVMSP-L9.6: SEAM MERGING FOR IMAGE RESIZING WITH STRUCTURE 1001 PRESERVATION

Kazu Mishiba, Masaaki Ikehara, Keio University, Japan

\section{IVMSP-P1: IMAGE FEATURE EXTRACTION}

IVMSP-P1.1: LOG-GAUSSIAN COX PROCESSES OF VISUAL KEYPOINTS FOR SONAR 1005 TEXTURE RECOGNITION

Huu-Giao Nguyen, Ronan Fablet, Jean-Marc Boucher, Université européenne de Bretagne, France

IVMSP-P1.2: SUPERVISED NONLINEAR SPECTRAL UNMIXING USING A POLYNOMIAL 1009 POST NONLINEAR MODEL FOR HYPERSPECTRAL IMAGERY

Yoann Altmann, Abderrahim Halimi, Nicolas Dobigeon, Jean-Yves Tourneret, University of Toulouse, France

IVMSP-P1.3: AN UNCONSTRAINED METHOD FOR LIP DETECTION IN COLOR IMAGES 1013

Evangelos Skodras, Nikolaos Fakotakis, University of Patras, Greece

IVMSP-P1.4: UNCOVERING ELEMENTS OF STYLE

Josephine Wolff, Massachusetts Institute of Technology, United States; Maximiliaan Martens, Ghent University, Belgium; Sina Jafarpour, Ingrid Daubechies, Princeton University, United States; Robert Calderbank, Duke University, United States

IVMSP-P1.5: AN ITERATIVE STRATEGY TO APPROACH CORNERS USING A NEW 1021 SALIENCY MEASUREMENT

Lihong Ma, Xingjun Tan, South China University of Technology, China; Jing Tian, Nanyang Technological University, Singapore

IVMSP-P1.6: DIRECTIONAL DESCRIPTORS USING ZERNIKE MOMENT PHASES FOR 1025 OBJECT ORIENTATION ESTIMATION IN UNDERWATER SONAR IMAGES

Naveen Kumar, Adam C. Lammert, University of Southern California, United States; Brendan Englot, Franz S. Hover, Massachusetts Institute of Technology, United States; Shrikanth S. Narayanan, University of Southern California, United States

IVMSP-P1.7: SORTING LOCAL DESCRIPTORS FOR LOW BIT RATE MOBILE VISUAL 1029 SEARCH

Jie Chen, Ling-Yu Duan, Peking University, China; Rongrong Ji, Hongxun Yao, Harbin Institute of Technology, China; Wen Gao, Peking University, China

IVMSP-P1.8: WAVELET DOMAIN DETECTION OF RUST IN STEEL BRIDGE IMAGES 1033

Sindhu Ghanta, Northeastern University, United States; Tanja Karp, Sangwook Lee, Texas Tech University, United States

IVMSP-P1.9: POLAR RANDOMIZED HOUGH TRANSFORM FOR LANE DETECTION 1037 USING LOOSE CONSTRAINTS OF PARALLEL LINES

Amol Borkar, Monson Hayes, Georgia Institute of Technology, United States; Mark Smith, KTH - Royal Institute of Technology, United States

IVMSP-P1.10: USING RESIDUAL VECTOR QUANTIZATION FOR IMAGE CONTENT 1041 CLASSIFICATION

Syed Irteza Ali Khan, Christopher F. Barnes, Georgia Institute of Technology, United States 


\section{IVMSP-P2: VIDEO CODING I}

IVMSP-P2.1: PREDICTION OF DISCRETE COSINE TRANSFORMED COEFFICIENTS IN

RESIZED PIXEL BLOCKS

Jin Li, Weiwei Chen, Moncef Gabbouj, Jarmo Takala, Tampere University of Technology, Finland; Hexin Chen, Jilin University, China

IVMSP-P2.2: REUSING THE H.264/AVC DEBLOCKING FILTER FOR EFFICIENT 1049 SPATIO-TEMPORAL PREDICTION IN VIDEO CODING

Jürgen Seiler, André Kaup, University of Erlangen-Nuremberg, Germany

IVMSP-P2.3: COMPRESSION USING SELF-SIMILARITY-BASED TEMPORAL 1053 SUPER-RESOLUTION FOR FULL-EXPOSURE-TIME VIDEO

Mihoko Shimano, University of Tokyo, Japan; Gene Cheung, Imari Sato, National Institute of Informatics, Japan

IVMSP-P2.4: A NEW ERROR RESILIENCE SCHEME BASED ON FMO AND ERROR 1057 CONCEALMENT IN H.264/AVC

Keyu Tan, Alan Pearmain Pearmain, Queen Mary University of London, United Kingdom

IVMSP-P2.5: ERROR DETECTION SCHEME BASED ON FRAGILE WATERMARKING FOR 1061 H.264/AVC

Man-Geun Ko, Jang-Eui Hong, Jae-Won Suh, Chungbuk National University, Republic of Korea

IVMSP-P2.6: SEAMLESS P2P-MDVC WITH WELL-BALANCED DESCRIPTIONS

Shuyuan Zhu, Siu-Kei Au Yeung, Bing Zeng, The Hong Kong University of Science and Technology, Hong Kong SAR of China

IVMSP-P2.7: HIGH PERFORMANCE DEARTIFACTING FILTERS IN VIDEO 1069 COMPRESSION

Renqi Zhang, The Chinese University of Hong Kong, Hong Kong SAR of China; Yu Liu, Hong Kong Applied Science and Technology Research Institute (ASTRI), Hong Kong SAR of China; Wai-Kuen Cham, The Chinese University of Hong Kong, Hong Kong SAR of China

IVMSP-P2.8: DOWN-SCALING INTERLACE VIDEO FROM H.264/AVC INTRA-CODED BIT 1073 STREAM

Huy Tran, Huyng Suk Oh, Wonha Kim, Kyung Hee University, Republic of Korea

\section{IVMSP-P3: IMAGE SEGMENTATION}

IVMSP-P3.1: AN ADAPTIVE BAYESIAN CLUSTERING AND MULTIVARIATE REGION 1077 MERGING BASED TECHNIQUE FOR EFFICIENT SEGMENTATION OF COLOR IMAGES

Sreenath Rao Vantaram, Eli Saber, Rochester Institute of Technology, United States

IVMSP-P3.2: LOW COMPLEXITY SHADOW REMOVAL ON FOREGROUND 1081 SEGMENTATION

Kazuki Nakagami, Toshiaki Shiota, Takao Nishitani, Tokyo Metropolitan University, Japan

IVMSP-P3.3: AN ATLAS-BASED DEEP BRAIN STRUCTURE SEGMENTATION METHOD: 1085 FROM COARSE POSITIONING TO FINE SHAPING

Yishan Luo, Albert C.S. Chung, The Hong Kong University of Science and Technology, Hong Kong SAR of China

IVMSP-P3.4: MARKER-BASED HIERARCHICAL SEGMENTATION AND CLASSIFICATION APPROACH FOR HYPERSPECTRAL IMAGERY

Yuliya Tarabalka, James C. Tilton, NASA Goddard Space Flight Center, United States; Jon Atli Benediktsson, University of Iceland, Iceland; Jocelyn Chanussot, Grenoble Institute of Technology, France 
IVMSP-P3.6: TEXTURE REMOVAL BY PIXEL CLASSIFICATION USING A ROTATING

\section{FILTER}

Baptiste Magnier, Philippe Montesinos, Daniel Diep, Ecole des Mines d'Alès, France

IVMSP-P3.7: SUPERPIXEL-BASED OBJECT CLASS SEGMENTATION USING

CONDITIONAL RANDOM FIELDS

Xi Li, Hichem Sahbi, CNRS / LTCI UMR 5141 / TELECOM ParisTech, France

\section{IVMSP-P4: RESTORATION, ENHANCEMENT, UPSAMPLING, AND SUPERRESOLUTION}

IVMSP-P4.1: ANGULAR REGULARIZATION OF VECTOR-VALUED SIGNALS. 1105

Kevin Holt, Varian Medical Systems, United States

IVMSP-P4.2: OPTICAL MEMS IMAGE ENHANCEMENT WITH SPARSE SIGNAL 1109 REPRESENTATION

Ganchi Zhang, Li Li, Vladimir Stankovic, Lina Stankovic, Deepak Uttamchandani, University of Strathclyde, United Kingdom

IVMSP-P4.3: EFFICIENT MATRIX COMPLETION WITH GAUSSIAN MODELS

Flavien Léger, ENS Cachan, France; Guoshen Yu, Guillermo Sapiro, University of Minnesota, United States

IVMSP-P4.4: ANTI-ALIASING FILTER FOR SUBPIXEL DOWN-SAMPLING BASED ON 1117 FREQUENCY ANALYSIS

Lu Fang, Ketan Tang, Oscar C. Au, The Hong Kong University of Science and Technology, Hong Kong SAR of China; Aggelos K. Katsaggelos, Northwestern University, Hong Kong SAR of China

IVMSP-P4.5: SSIM-INSPIRED IMAGE DENOISING USING SPARSE REPRESENTATIONS 1121

Abdul Rehman, Zhou Wang, Dominique Brunet, Edward Vrscay, University of Waterloo, Canada

IVMSP-P4.6: VIDEO ERROR CONCEALMENT USING SPARSE RECOVERY AND LOCAL 1125 DICTIONARIES

Dzung Nguyen, Minh Dao, Trac Tran, The Johns Hopkins University, United States

IVMSP-P4.7: DIFFERENCE IMAGE EXTRAPOLATION FOR SPECTRAL COMPLETION IN 1129 INTER-SEQUENCE ERROR CONCEALMENT

Tobias Tröger, André Kaup, University of Erlangen-Nuremberg, Germany

IVMSP-P4.8: REGULARIZED SPLIT GRADIENT METHOD FOR NONNEGATIVE MATRIX FACTORIZATION

Henri Lanteri, Celine Theys, Cédric Richard, David Mary, Université de Nice Sophia-Antipolis, France

IVMSP-P4.9: FULLY NON-LOCAL SUPER-RESOLUTION VIA SPECTRAL HASHING

Emmanuel d'Angelo, Pierre Vandergheynst, Ecole Polytechnique Fédérale de Lausanne, Switzerland

IVMSP-P4.10: A NEW IMAGE DENOISING METHOD BASED ON THE WAVELET DOMAIN 1141 NONLOCAL MEANS FILTERING

Su Jeong You, Nam Ik Cho, Seoul National University, Republic of Korea

\section{IVMSP-P5: IMAGE AND VIDEO QUALITY ASSESSMENT}

IVMSP-P5.1: STRUCTURAL SIMILARITY INDICES FOR HIGH DYNAMIC RANGE 1145 IMAGING

Zhengguo Li, Zijian Zhu, Susanto Rahardja, Institute for Infocomm Research, Singapore

IVMSP-P5.2: RRED INDICES: REDUCED REFERENCE ENTROPIC DIFFERENCING 1149 FRAMEWORK FOR IMAGE QUALITY ASSESSMENT

Rajiv Soundararajan, Alan Bovik, The University of Texas at Austin, United States 
VIDEO QUALITY

Kalpana Seshadrinathan, Intel Corporation, United States; Alan Bovik, The University of Texas at Austin, United States

IVMSP-P5.4: ADAPTIVE RECONSTRUCTION METHOD OF MISSING TEXTURES BASED 1157 ON PERCEPTUALLY OPTIMIZED ALGORITHM

Takahiro Ogawa, Miki Haseyama, Hokkaido University, Japan

IVMSP-P5.5: CYCLOP: A STEREO COLOR IMAGE QUALITY ASSESSMENT METRIC 1161

Aldo Maalouf, Mohamed Chaker Larabi, University of Poitiers, France

IVMSP-P5.6: VIDEO AESTHETIC QUALITY ASSESSMENT BY COMBINING 1165 SEMANTICALLY INDEPENDENT AND DEPENDENT FEATURES

Chun-Yu Yang, Hsin-Ho Yeh, Chu-Song Chen, Academia Sinica, Taiwan

IVMSP-P5.7: NO-REFERENCE BIT STREAM MODEL FOR VIDEO QUALITY 1169 ASSESSMENT OF H.264/AVC VIDEO BASED ON PACKET LOSS VISIBILITY

Savvas Argyropoulos, Alexander Raake, Marie-Neige Garcia, Peter List, Deutsche Telekom Laboratories, Germany

\section{IVMSP-P6: VIDEO SEGMENTATION AND TRACKING}

IVMSP-P6.1: RAO-BLACKWELLIZED PARTICLE FILTER FOR GAUSSIAN MIXTURE 1173 MODELS AND APPLICATION TO VISUAL TRACKING

Jungho Kim, In So Kweon, Korea Advanced Institute of Science and Technology, Republic of Korea

IVMSP-P6.2: AN EFFECTIVE FOREGROUND/BACKGROUND SEGMENTATION APPROACH 1177 FOR BOOTSTRAPPING VIDEO SEQUENCES

Han-Hui Hsiao, Jin-Jang Leou, National Chung Cheng University, Taiwan

IVMSP-P6.3: A GENERAL BAYESIAN ALGORITHM FOR VISUAL OBJECT TRACKING 1181 BASED ON SPARSE FEATURES

Mauricio Soto Alvarez, Carlo S. Regazzoni, University of Genoa, Italy

IVMSP-P6.4: MULTI-CUE BASED MULTI-TARGET TRACKING USING ONLINE RANDOM 1185 FORESTS

Xinchu Shi, Institute of Automation / Chinese Academy of Sciences, China; Xiaoqin Zhang, Wenzhou university, China; Yang Liu, Weiming Hu, Institute of Automation / Chinese Academy of Sciences, China; Haibin Ling, Temple University, United States

IVMSP-P6.5: GRAPH-BASED SEQUENTIAL PARTICLE FILTERING IN LOSSY 1189 NETWORKS: SINGLE AND MULTIPLE COLLABORATIVE CAMERAS

Jing Huang, Dan Schonfeld, University of Illinois Chicago, United States

IVMSP-P6.6: MULTIPLE INSTANCE TRACKING BASED ON HIERARCHICAL MAXIMIZING 1193 BAG'S MARGIN BOOSTING

Chunxiao Liu, Guijin Wang, Xinggang Lin, Bobo Zeng, Tsinghua University, China

IVMSP-P6.7: A ROBUST LIP TRACKING ALGORITHM USING LOCALIZED COLOR 1197 ACTIVE CONTOURS AND DEFORMABLE MODELS

Xin Liu, Yiu-ming Cheung, Hong Kong Baptist University, Hong Kong SAR of China

IVMSP-P6.8: A NEW VIDEO SIMILARITY MEASURE MODEL BASED ON VIDEO TIME 1201 DENSITY FUNCTION AND DYNAMIC PROGRAMMING

Junfeng Jiang, Xiao-Ping Zhang, Ryerson University, Canada; Alexander C. Loui, Kodak Company, United States

IVMSP-P6.9: EFFICIENT BLOCK-DIVISION MODEL FOR ROBUST MULTIPLE OBJECT 1205 TRACKING

Wenhan Luo, National Laboratory of Pattern Recognition / Institute of Automation / Chinese Academy of Sciences, China; Xiaoqin Zhang, Wenzhou University, China; Yang Liu, National Laboratory of Pattern Recognition / Institute of Automation / Chinese Academy of Sciences, China; Xi Li, University of Adelaide, Australia; Weiming Hu, Wei Li, National Laboratory of Pattern Recognition / Institute of Automation / Chinese Academy of Sciences, China 


\section{IVMSP-P7: IMAGE ANALYSIS}

IVMSP-P7.1: KEYPOINT-BASED NEAR-DUPLICATE IMAGES DETECTION USING AFFINE

INVARIANT FEATURE AND COLOR MATCHING

Yue Wang, ZuJun Hou, Karianto Leman, Institute for Infocomm Research / Agency of Science Technology And Research,

Singapore

IVMSP-P7.2: REGION-BASED IMAGE FUSION USING A COMBINATORY

1213 CHEBYSHEV-ICA METHOD

Zaid Omar, Nikolaos Mitianoudis, Tania Stathaki, Imperial College London, United Kingdom

IVMSP-P7.3: A SEGMENT-BASED IMAGE SALIENCY DETECTION

Oleg Muratov, Pamela Zontone, Giulia Boato, Francesco G. B. De Natale, University of Trento, Italy

IVMSP-P7.4: MULTI-STAGE INFRARED STATIONARY HUMAN DETECTION

Alex Chan, U.S. Army Research Laboratory, United States

IVMSP-P7.5: AUTOMATIC TARGET CLASSIFICATION IN SAR IMAGES USING MPCA

Tristan Porgès, Gérard Favier, Laboratoire i3s, France

IVMSP-P7.6: GENERIC OBJECT RECOGNITION USING AUTOMATIC REGION

EXTRACTION AND DIMENSIONAL FEATURE INTEGRATION UTILIZING MULTIPLE KERNEL

LEARNING

Toru Nakashika, Akira Suga, Tetsuya Takiguchi, Yasuo Ariki, Kobe University, Japan

IVMSP-P7.7: DETECTION OF ELLIPTICAL PARTICLES IN ATOMIC FORCE

1233 MICROSCOPY IMAGES

Jirí Sedlár, Barbara Zitová, Institute of Information Theory and Automation, Czech Republic; Jaromír Kopecek, Tatiana

Todorciuc, Irena Kratochvílová, Academy of Sciences of the Czech Republic, Czech Republic

IVMSP-P7.8: ON TUNING OF SELF-QUOTIENT EPSILON-FILTER AND SUPPORT 1237 VECTOR MACHINE AND ITS APPLICATION TO NOISE ROBUST HUMAN DETECTION

Mitsuharu Matsumoto, University of Electro-Communications, Japan

IVMSP-P7.9: SUPERRESOLUTION AND SUPERFAST RECEIVERS IN FREE-SPACE RADAR

IMAGING

Ricardo Merched, Universidade Federal do Rio de Janeiro, Brazil

\section{IVMSP-P8: IMAGE/VIDEO ENHANCEMENT}

IVMSP-P8.1: FAST SINGLE IMAGE FOG REMOVAL USING EDGE-PRESERVING 1245 SMOOTHING

Jing Yu, Qingmin Liao, Tsinghua University, China

IVMSP-P8.2: ENHANCEMENT OF UNEVEN LIGHTING TEXT IMAGE USING 1249 LINE-BASED EMPIRICAL MODE DECOMPOSITION

Soo-Chang Pei, Mary Tzeng, Yu-Zhe Hsiao, National Taiwan University, Taiwan

IVMSP-P8.3: ON THE EFFECTIVENESS OF THE DARK CHANNEL PRIOR FOR SINGLE 1253 IMAGE DEHAZING BY APPROXIMATING WITH MINIMUM VOLUME ELLIPSOIDS

Kristofor Gibson, Truong Q. Nguyen, University of California San Diego, United States

IVMSP-P8.4: SPARSITY-BASED DEFECT PIXEL COMPENSATION FOR ARBITRARY 1257 CAMERA RAW IMAGES

Michael Schöberl, Jürgen Seiler, University of Erlangen-Nuremberg, Germany; Bernhard Kasper, Siegfried Foessel, Fraunhofer Institute for Integrated Circuits (IIS), Germany; André Kaup, University of Erlangen-Nuremberg, Germany 
EQUATION

Zhaozhong Wang, Beihang University, China

IVMSP-P8.6: DUAL DOMAIN METHOD FOR SINGLE IMAGE DEHAZING AND

ENHANCING

Dongin Shin, Kyung Hee University, Republic of Korea; Kristofor Gibson, University of California San Diego, United States;

Wonha Kim, Kyung Hee University, Republic of Korea; Truong Q. Nguyen, University of California San Diego, United States

IVMSP-P8.7: LOCALIZED FILTERING FOR ARTIFACT REMOVAL IN COMPRESSED 1269

IMAGES

Dung Vo, Truong Q. Nguyen, University of California San Diego, United States

IVMSP-P8.8: SINGLE IMAGE DEHAZING BASED ON CONTRAST ENHANCEMENT 1273

Jin-Hwan Kim, Korea University, Republic of Korea; Jae-Young Sim, UNIST, Republic of Korea; Chang-Su Kim, Korea

University, Republic of Korea

\section{IVMSP-P9: IMAGE AND VIDEO MODELING AND APPLICATIONS}

IVMSP-P9.1: LEARNING AND INFERENCE ALGORITHMS FOR PARTIALLY OBSERVED STRUCTURED SWITCHING VECTOR AUTOREGRESSIVE MODELS

Balakrishnan Varadarajan, Sanjeev Khudanpur, The Johns Hopkins University, United States

IVMSP-P9.2: A NEW STOCHASTIC IMAGE MODEL BASED ON MARKOV RANDOM 1285 FIELDS AND ITS APPLICATION TO TEXTURE MODELING

Siamak Yousefi, Nasser Kehtarnavaz, The University of Texas at Dallas, United States

IVMSP-P9.3: LOCAL PROBABILITY DISTRIBUTION OF NATURAL SIGNALS IN SPARSE 1289 DOMAINS

Hossein Rabbani, Isfahan University of Medical Sciences, Iran; Saeed Gazor, Queen's University Belfast, Canada

IVMSP-P9.4: A VISUAL ATTENTION MODEL COMBINING TOP-DOWN AND BOTTOM-UP 1293 MECHANISMS FOR SALIENT OBJECT DETECTION

Yuming Fang, Weisi Lin, Chiew Tong Lau, Bu-Sung Lee, Nanyang Technological University, Singapore

IVMSP-P9.5: HIERARCHICAL LATENT DIRICHLET ALLOCATION MODELS FOR 1297 REALISTIC ACTION RECOGNITION

Heping Li, Jie Liu, Shuwu Zhang, Institute of Automation / Chinese Academy of Sciences, China

IVMSP-P9.6: PARAMETRIC MODELING AND LINEAR ESTIMATION OF ELASTIC 1301 DEFORMATIONS

Nadav Geva, Rami Hagege, Joseph M. Francos, Ben-Gurion University of the Negev, Israel

IVMSP-P9.7: SPIRAL COLOUR MODEL: REDUCTION FROM 3-D TO 2-D

Frederic Garcia, University of Luxembourg / IEE S.A., Luxembourg; Djamila Aouada, University of Luxembourg, Luxembourg;

Bruno Mirbach, IEE S.A., Luxembourg; Björn Ottersten, University of Luxembourg, Luxembourg

IVMSP-P9.8: QUADRATIC OPTIMIZATION BASED SMALL SCALE DETAILS EXTRACTION 1309

Zhengguo Li, Jinghong Zheng, Chuohao Yeo, Susanto Rahardja, Institute for Infocomm Research, Singapore

IVMSP-P9.9: VIDEO PROCESSING WITH SCALE-AWARE SALIENCY: APPLICATION TO 1313 FRAME RATE UP-CONVERSION

Natan Jacobson, Truong Q. Nguyen, University of California San Diego, United States 


\section{IVMSP-P10: VIDEO, IMAGE, AND 3D ANALYSIS}

IVMSP-P10.1: VIDEO ANOMALY RECOVERY FROM COMPRESSED SPECTRAL IMAGING 1321

Ana Ramirez, Henry Arguello, Gonzalo R. Arce, University of Delaware, United States

IVMSP-P10.2: HORROR VIDEO SCENE RECOGNITION VIA MULTIPLE-INSTANCE

\section{LEARNING}

Jianchao Wang, Bing Li, Weiming Hu, Ou Wu, National Laboratory of Pattern Recognition / Institute of Automation / Chinese Academy of Sciences, China

IVMSP-P10.3: SPARSE VIDEO RECOVERY USING LINEARLY CONSTRAINED GRADIENT 1329 PROJECTION

Daniel Thompson, University of California Merced, United States; Zachary Harmany, Duke University, United States; Roummel Marcia, University of California Merced, United States

IVMSP-P10.4: MOTION-DECISION BASED SPATIOTEMPORAL SALIENCY FOR VIDEO 1333 SEQUENCES

Yaping Zhu, Communication University of China, China; Natan Jacobson, Hong Pan, Truong Q. Nguyen, University of California San Diego, United States

IVMSP-P10.5: DETECTING HUMAN ACTIVITIES IN RETAIL SURVEILLANCE USING 1337 HIERARCHICAL FINITE STATE MACHINE

Hoang Trinh, Quanfu Fan, Pan Jiyan, Prasad Gabbur, Sachiko Miyazawa, Sharath Pankanti, IBM, United States

IVMSP-P10.6: A NOVEL VECTOR QUANTIZATION-BASED VIDEO SUMMARIZATION 1341 METHOD USING INDEPENDENT COMPONENT ANALYSIS MIXTURE MODEL

Junfeng Jiang, Xiao-Ping Zhang, Ryerson University, Canada

IVMSP-P10.7: DETECTION AND REMOVAL OF BINOCULAR LUSTER IN COMPRESSED 1345 3D IMAGES

Can Bal, Ankit Kumar Jain, Truong Q. Nguyen, University of California San Diego, United States

IVMSP-P10.8: ERROR COMPENSATION AND RELIABILITY BASED VIEW SYNTHESIS.

Wenxiu Sun, Oscar C. Au, Lingfeng Xu, Sung Him Chui, Chun Wing Kwok, Yujun Li, The Hong Kong University of Science and Technology, Hong Kong SAR of China

IVMSP-P10.9: COMPRESSED CLASSIFICATION OF OBSERVATION SETS WITH LINEAR 1353 SUBSPACE EMBEDDINGS

Dorina Thanou, Pascal Frossard, Ecole Polytechnique Fédérale de Lausanne, Switzerland

IVMSP-P10.10: RANDOM FINITE SET FOR DATA ASSOCIATION IN MULTIPLE CAMERA 1357 TRACKING

Nam Trung Pham, Richard Chang, Karianto Leman, Teck Wee Chua, Yue Wang, Institute for Infocomm Research, Singapore

\section{IVMSP-P11: OPTICAL IMAGING AND REMOTE SENSING}

IVMSP-P11.1: ROBUST HYPERSPECTRAL SIGNAL UNMIXING IN THE PRESENCE OF 1361 CORRELATED NOISE

Masoud Farzam, Soosan Beheshti, Ryerson University, Canada

IVMSP-P11.2: CLEAN: A FALSE ALARM REDUCTION METHOD FOR SAR CCD 1365

Rhonda D. Phillips, MIT Lincoln Laboratory, United States 
IVMSP-P11.3: TWO EFFECTIVE AND COMPUTATIONALLY EFFICIENT PURE-PIXEL 1369 BASED ALGORITHMS FOR HYPERSPECTRAL ENDMEMBER EXTRACTION

ArulMurugan Ambikapathi, Tsung-Han Chan, Chong-Yung Chi, Kannan Keizer, National Tsing Hua University, Taiwan

IVMSP-P11.4: IMPROVED SECONDARY RANGE COMPRESSION FOCUSING METHOD 1373 IN GEO SAR

Zhipeng Liu, Cheng Hu, Tao Zeng, Beijing Institute of Technology, China

IVMSP-P11.5: MAXIMUM LIKELIHOOD SAR AUTOFOCUS WITH LOW-RETURN REGION 1377 Kuang-Hung Liu, University of Michigan Ann Arbor, United States; Ami Wiesel, Hebrew University of Jerusalem, Israel; David Munson, University of Michigan Ann Arbor, United States

IVMSP-P11.6: OIL SPILL SENSOR USING MULTISPECTRAL INFRARED IMAGING VIA L1 1381 MINIMIZATION

Yingying Li, Wei-chuan Shih, Zhu Han, University of Houston, United States; Wotao Yin, Rice University, United States

IVMSP-P11.7: SATURATION-ROBUST SAR IMAGE FORMATION

Dennis Wei, Massachusetts Institute of Technology, United States; Petros Boufounos, Mitsubishi Electric Research Laboratories, United States

IVMSP-P11.8: QUADRATURE APPROXIMATION PROPERTIES OF THE SPIRAL-PHASE 1389 QUADRATURE TRANSFORM

Haricharan Aragonda, Indian Institute of Science, India; Chandra Sekhar Seelamantula, Indian Institute of science, India

IVMSP-P11.9: A POINT TARGET REFERENCE SPECTRUM FOR GENERAL BISTATIC SAR 1393 PROCESSING

Junjie Wu, Jianyu Yang, Yulin Huang, Haiguang Yang, Zhe Liu, University of Electronic Science and Technology of China, China

\section{IVMSP-P12: VIDEO TRACKING}

IVMSP-P12.1: A SPARSE AND LOW-RANK APPROACH TO EFFICIENT FACE ALIGNMENT 1397 FOR PHOTO-REAL TALKING HEAD SYNTHESIS

King Keung Wu, The Chinese University of Hong Kong, Hong Kong SAR of China; Lijuan Wang, Frank K. Soong, Microsoft Research Asia, China; Yeung Yam, The Chinese University of Hong Kong, Hong Kong SAR of China

IVMSP-P12.2: SIMULTANEOUS OBJECT TRACKING AND DEPTH ESTIMATION USING 1401 COLOR SHIFTING PROPERTY OF A MULTIPLE COLOR-FILTER APERTURE CAMERA Seungwon Lee, Jinhee Lee, Joonki Paik, Chung-Ang University, Republic of Korea

IVMSP-P12.3: VIDEO OBJECT TRACKING WITH DIFFERENTIAL STRUCTURAL 1405 SIMILARITY INDEX

Artur Loza, Fanglin Wang, Jie Yang, Shanghai Jiao Tong University, China; Lyudmila Mihaylova, Lancaster University, United Kingdom

IVMSP-P12.4: MULTI-OBJECT TRACKING VIA HIGH ACCURACY OPTICAL FLOW AND 1409 FINITE SET STATISTICS

Marek Schikora, Wolfgang Koch, Fraunhofer FKIE, Germany; Daniel Cremers, Technical University of Munich, Germany

IVMSP-P12.5: ADAPTIVE APPEARANCE LEARNING FOR VISUAL OBJECT TRACKING 1413

Zulfiqar Khan, Irene Y.H. Gu, Chalmers University of Technology, Sweden

IVMSP-P12.6: VIDEO THUMBNAIL EXTRACTION USING VIDEO TIME DENSITY

FUNCTION AND INDEPENDENT COMPONENT ANALYSIS MIXTURE MODEL

Junfeng Jiang, Xiao-Ping Zhang, Ryerson University, Canada

IVMSP-P12.7: ROBUST VIDEO OBJECT TRACKING BASED ON MULTIPLE KERNELS 1421 WITH PROJECTED GRADIENTS

Chun-Te Chu, Jenq-Neng Hwang, University of Washington, United States; Hung-I Pai, Kung-Ming Lan, Industrial Technology Research Institute, Taiwan 


\section{SYSTEMS}

Chih-Chang Chen, Hsing-Hao Lin, Oscal T.-C. Chen, National Chung Cheng University, Taiwan

IVMSP-P12.9: ULTRA-FAST TRACKING BASED ON ZERO-SHIFT POINTS

Jan Dupac, RS Dynamics s. r. o., Czech Republic; Jirí Matas, Czech Technical University, Czech Republic

\section{IVMSP-P13: IMAGE FILTERING}

IVMSP-P13.1: IMAGE FILTERING: POTENTIAL EFFICIENCY AND CURRENT PROBLEMS 1433 Vladimir Lukin, Sergey Abramov, Nikolay Ponomarenko, National Aerospace University, Ukraine; Karen Egiazarian, Jaakko Astola, Tampere University of Technology, Finland

IVMSP-P13.2: MEDIAN FILTER WITH ABSOLUTE VALUE NORM SPATIAL 1437 REGULARIZATION

Nilanjan Ray, University of Alberta, Canada

IVMSP-P13.3: LEARNING SPARSE DICTIONARIES WITH A POPULARITY-BASED MODEL

Jianzhou Feng, Li Song, Shanghai Jiaotong University, China; Xiaoming Huo, Georgia Institute of Technology, China; Xiaokang Yang, Wenjun Zhang, Shanghai Jiaotong University, China

IVMSP-P13.4: BAYESIAN DESPECKLING OF SAR IMAGES BASED ON LAPLACIAN-GAUSSIAN 1445 MODELING OF UNDECIMATED WAVELET COEFFICIENTS

Fabrizio Argenti, Tiziano Bianchi, Alessandro Lapini, Luciano Alparone, University of Florence, Italy

IVMSP-P13.5: SEAM CARVING WITH RATE-DEPENDENT SEAM PATH INFORMATION 1449

Yuichi Tanaka, Madoka Hasegawa, Shigeo Kato, Utsunomiya University, Japan

IVMSP-P13.6: SINGLE-FRAME-BASED RAIN REMOVAL VIA IMAGE DECOMPOSITION 1453 Yu-Hsiang Fu, National Tsing Hua University, Taiwan; Li-Wei Kang, Academia Sinica, Taiwan; Chia-Wen Lin, Chiou-Ting Hsu, National Tsing Hua University, Taiwan

IVMSP-P13.7: LINEAR OPENINGS IN ARBITRARY ORIENTATION IN O(1) PER PIXEL 1457 Vincent Morard, Petr Dokladal, Etienne Decencière, MINES PARISTECH, France

IVMSP-P13.8: HIGH DYNAMIC RANGE (HDR) IMAGING BY GRADIENT DOMAIN FUSION. 1461 Jung Gap Kuk, Nam Ik Cho, Sang Uk Lee, Seoul National University, Republic of Korea

IVMSP-P13.9: SNAPSHOT SPECTRAL IMAGING VIA COMPRESSIVE RANDOM 1465 CONVOLUTION

Yao Wu, Gonzalo R. Arce, University of Delaware, United States

\section{IVMSP-P14: BIOMETRIC AND FACE IMAGE PROCESSING}

IVMSP-P14.1: A ROBUST FEATURE EXTRACTION ALGORITHM BASED ON 1469 CLASS-MODULAR IMAGE PRINCIPAL COMPONENT ANALYSIS FOR FACE VERIFICATION

José Francisco Pereira, Rafael M. Barreto, George D. C. Cavalcanti, Ing Ren Tsang, Federal University of Pernambuco, Brazil

IVMSP-P14.2: A NOVEL STUDY AND ANALYSIS ON SEGMENTAL GAIT SEQUENCE 1473 RECOGNITION

Nini Liu, Yap-Peng Tan, Nanyang Technological University, Singapore

IVMSP-P14.3: FUSING SHAPE AND TEXTURE INFORMATION FOR FACIAL AGE 1477 ESTIMATION

Jiwen Lu, Yap-Peng Tan, Nanyang Technological University, Singapore

IVMSP-P14.4: ROBUST TALKING FACE VIDEO VERIFICATION USING JOINT FACTOR 1481 ANALYSIS AND SPARSE REPRESENTATION ON GMM MEAN SHIFTED SUPERVECTORS Ming Li, Shrikanth S. Narayanan, University of Southern California, United States 
Thiago Zavaschi, PUCPR, Brazil; Alessandro Koerich, PUCPR / UFPR, Brazil; Luiz Eduardo Oliveira, Federal University of Paraná, Brazil

IVMSP-P14.7: INCREMENTAL TWO-DIMENSIONAL TWO-DIRECTIONAL PRINCIPAL 1493 COMPONENT ANALYSIS (I(2D)2PCA) FOR FACE RECOGNITION

Yonghwa Choi, Kyungpook National University, Republic of Korea; Takaomi Tokumoto, Kobe University, Japan; Minho Lee, Kyungpook National University, Republic of Korea; Seiichi Ozawa, Kobe University, Japan

IVMSP-P14.8: COMBINING HAAR FEATURE AND SKIN COLOR BASED CLASSIFIERS FOR 1497 FACE DETECTION

Cigdem Eroglu Erdem, Sezer Ulukaya, Ali Karaali, Bahcesehir University, Turkey; Tanju Erdem, Ozyegin University, Turkey

IVMSP-P14.9: BEMD FOR EXPRESSION TRANSFORMATION IN FACE RECOGNITION.

Hoda Mohammadzade, Foteini Agrafioti, Jiexin Gao, Dimitrios Hatzinakos, University of Toronto, Canada

\section{IVMSP-P15: IMAGE AND VIDEO CODING}

IVMSP-P15.1: CORRELATION ESTIMATION WITH PARTICLE-BASED BELIEF 1505 PROPAGATION FOR DISTRIBUTED VIDEO CODING

Lina Stankovic, Vladimir Stankovic, University of Strathclyde, United Kingdom; Shuang Wang, Samuel Cheng, University of Oklahoma, United States

IVMSP-P15.2: A DISTANCE-BASED SLICE INTERLEAVING SCHEME FOR ROBUST 1509 VIDEO TRANSMISSION OVER ERROR-PRONE NETWORKS

Yu Wang, Jo Yew Tham, Kwong Huang Goh, Wei Siong Lee, Wenxian Yang, Institute for Infocomm Research / Agency of Science Technology And Research, Singapore

IVMSP-P15.3: A CLASSIFIER-BASED DECODING APPROACH FOR LARGE SCALE 1513 DISTRIBUTED CODING

Kumar Viswanatha, Sharadh Ramaswamy, Ankur Saxena, Kenneth Rose, University of California Santa Barbara, United States

IVMSP-P15.4: IMAGE COMPRESSION USING LEARNED DICTIONARIES BY RLS-DLA AND 1517 COMPARED WITH K-SVD

Karl Skretting, Kjersti Engan, University of Stavanger, Norway

IVMSP-P15.5: MULTIPLE LDPC DECODING USING BITPLANE CORRELATION FOR 1521 TRANSFORM DOMAIN WYNER-ZIV VIDEO CODING

Huynh Van Luong, Xin Huang, Søren Forchhammer, Technical University of Denmark, Denmark

IVMSP-P15.6: INTEGER FAST LAPPED ORTHOGONAL TRANSFORM BASED ON 1525 DIRECT-LIFTING OF DCTS FOR LOSSLESS-TO-LOSSY IMAGE CODING

Taizo Suzuki, Masaaki Ikehara, Keio University, Japan

IVMSP-P15.7: TWO DIMENSIONAL NON-SEPARABLE ADAPTIVE DIRECTIONAL LIFTING 1529 STRUCTURE OF DISCRETE WAVELET TRANSFORM

Taichi Yoshida, Taizo Suzuki, Keio University, Japan; Seisuke Kyochi, NTT Corporation, Japan; Masaaki Ikehara, Keio University, Japan

IVMSP-P15.8: JOINT SOURCE-CHANNEL RATE CONTROL FOR PIXEL-DOMAIN 1533 DISTRIBUTED VIDEO CODING

Hu Chen, Eckehard Steinbach, Technische Universität München, Germany; Chang Wen Chen, State University of New York at Buffalo, United States 
ARITHMETIC CODING OF VIDEO TEXTURE

Kenneth Vermeirsch, Ghent University, Belgium; Joeri Barbarien, Vrije Universiteit Brussel, Belgium; Peter Lambert, Rik Van de Walle, Ghent University, Belgium

IVMSP-P15.10: PERFORMANCE OPTIMIZED PREDICTOR BLENDING TECHNIQUE FOR 1541 LOSSLESS IMAGE CODING

Grzegorz Ulacha, West Pomeranian University of Technology, Poland; Ryszard Stasinski, Poznan University of Technology,

Poland

\section{IVMSP-P16: MOTION ESTIMATION AND REGISTRATION}

IVMSP-P16.1: ALIGNMENT OF CUBIC-PANORAMA IMAGE DATASETS USING EPIPOLAR 1545 GEOMETRY

Saeed Salehi, Eric Dubois, University of Ottawa, Canada

IVMSP-P16.2: MRF-BASED AUTOMATIC IMAGE ORDERING AND ITS APPLICATION TO 1549 MOSAICING

Ran Song, Yonghuai Liu, Yitian Zhao, Aberystwyth University, United Kingdom; Ralph Martin, Paul Rosin, Cardiff University, United Kingdom

IVMSP-P16.4: TOF-CCD IMAGE FUSION USING COMPLEX WAVELETS 1557

Sigurjon Arni Gudmundsson, Johannes R. Sveinsson, University of Iceland, Iceland

IVMSP-P16.5: FEATURE-BASED GLOBAL MOTION ESTIMATION USING THE 1561 HELMHOLTZ PRINCIPLE

Michael Tok, Alexander Glantz, Andreas Krutz, Thomas Sikora, Technische Universität Berlin, Germany

IVMSP-P16.6: A MULTI-EXPOSURE IMAGE FUSION ALGORITHM WITHOUT GHOST 1565 EFFECT

Jaehyun An, Sang Heon Lee, Jung Gap Kuk, Nam Ik Cho, Seoul National University, Republic of Korea

IVMSP-P16.7: A NOVEL ALGORITHM FOR OCCLUSIONS AND PERSPECTIVE EFFECTS 1569 USING A 3D OBJECT PROCESS

Ahmed Gamal-Eldin, Xavier Descombes, Josiane Zerubia, INRIA Sophia-Antipolis Mediterannee, France

IVMSP-P16.8: 3D IMAGE GEO-REGISTRATION USING VISION-BASED MODELING

Karl Ni, Zachary Sun, Nadya Bliss, MIT Lincoln Laboratory, United States

\section{DISPS-L1: DSP ALGORITHM AND ARCHITECTURE OPTIMIZATION FOR HARDWARE IMPLEMENTATION}

DISPS-L1.1: JOINT ALGORITHM-ARCHITECTURE OPTIMIZATION OF CABAC TO 1577 INCREASE SPEED AND REDUCE AREA COST

Vivienne Sze, Anantha Chandrakasan, Massachusetts Institute of Technology, United States

DISPS-L1.2: NEW RADIX-BASED FHT ALGORITHM FOR COMPUTING THE DISCRETE 1581 HARTLEY TRANSFORM

Monir Taha Hamood, Said Boussakta, Newcastle University, United Kingdom

DISPS-L1.3: MULTI-RATE POLYPHASE DSP AND LMS CALIBRATION SCHEMES FOR 1585 OVERSAMPLED DATA CONVERSION SYSTEMS

Subhanshu Gupta, University of Washington, United States; Yi Tang, Qualcomm Inc., Singapore; Kuang-Wei Cheng, Institute of Microelectronics, Singapore; Jeyanandh Paramesh, Carnegie Mellon University, United States; David Allstot, University of Washington, United States 
LOW POWER JPEG IMPLEMENTATION

Yunus Emre, Chaitali Chakrabarti, Arizona State University, United States

DISPS-L1.5: A LOW-POWER IMPLANTABLE NEUROPROCESSOR ON NANO-FPGA FOR 1593

BRAIN MACHINE INTERFACE APPLICATIONS

Fei Zhang, Mehdi Aghagolzadeh, Karim Oweiss, Michigan State University, United States

DISPS-L1.6: IMPROVING KERNEL-ENERGY TRADE-OFFS FOR MACHINE LEARNING IN 1597 IMPLANTABLE AND WEARABLE BIOMEDICAL APPLICATIONS

Kyong Ho Lee, Sun-Yuan Kung, Naveen Verma, Princeton University, United States

\section{DISPS-L2: PARALLEL SOFTWARE IMPLEMENTATION OF DSP ALGORITHMS}

DISPS-L2.1: PARALLEL COMPUTATION OF ADAPTIVE LATTICE FILTERS. 1601

Dong-hwan Lee, Wonyong Sung, Seoul National University, Republic of Korea

DISPS-L2.2: HETEROGENEOUS MULTIPROCESSOR MAPPING FOR REAL-TIME STREAMING SYSTEMS

Jing Lin, The University of Texas at Austin, United States; Akshaya Srivatsa, Rice University, United States; Andreas Gerstlauer, Brian Evans, The University of Texas at Austin, United States

DISPS-L2.3: SCHEDULING OF CAL ACTOR NETWORKS BASED ON DYNAMIC CODE 1609 ANALYSIS

Jani Boutellier, University of Oulu, Finland; Mickaël Raulet, INSA Rennes, France; Olli Silvén, University of Oulu, Finland

DISPS-L2.4: A METHODOLOGY BASED ON TRANSPORTATION PROBLEM MODELING 1613 FOR DESIGNING PARALLEL INTERLEAVER ARCHITECTURES

Awais Hussain Sani, Philippe Coussy, Cyrille Chavet, Eric Martin, Université de Bretagne Sud / Lab-STICC, France

DISPS-L2.5: DART - A HIGH LEVEL SOFTWARE-DEFINED RADIO PLATFORM MODEL 1617 FOR DEVELOPING THE RUN-TIME CONTROLLER

Martin Palkovic, Jeroen Declerck, Praveen Raghavan, Antoine Dejonghe, Liesbet Van der Perre, IMEC, Belgium

DISPS-L2.6: PARALLEL IMPLEMENTATION OF MULTI-DIMENSIONAL ENSEMBLE 1621 EMPIRICAL MODE DECOMPOSITION

Li-Wen Chang, University of Illinois Urbana-Champaign, United States; Men-Tzung Lo, National Central University, Taiwan; Nasser Anssari, University of Illinois Urbana-Champaign, United States; Ke-Hsin Hsu, Norden Huang, National Central University, Taiwan; Wen-mei Hwu, University of Illinois Urbana-Champaign, United States

DISPS-P1: DSP ALGORITHM AND FILTER DESIGN

DISPS-P1.1: OPENBLISSART: DESIGN AND EVALUATION OF A RESEARCH TOOLKIT FOR 1625 BLIND SOURCE SEPARATION IN AUDIO RECOGNITION TASKS

Felix Weninger, Alexander Lehmann, Björn Schuller, Technische Universität München, Germany

DISPS-P1.2: A NEW VARIABLE DIGITAL FILTER DESIGN BASED ON FRACTIONAL DELAY 1629

Sumit Darak, Vinod Prasad, Nanyang Technological University, Singapore; Edmund Lai, Massey University, New Zealand

DISPS-P1.3: DESIGN AND ANALYSIS OF A NARROWBAND FILTER FOR OPTICAL 1633 PLATFORM

Yujia Wang, Andrew Grieco, Boris Slutsky, Bhaskar D. Rao, Yeshaiahu Fainman, Truong Q. Nguyen, University of California San Diego, United States

DISPS-P1.4: A NOVEL FAST CANONICAL-SIGNED-DIGIT CONVERSION TECHNIQUE 1637 FOR MULTIPLICATION

Rui Guo, Linda DeBrunner, Florida State University, United States 
SPIKE SORTING MICROSYSTEMS

Tung-Chien Chen, Yun-Yu Chen, Tsung-Chuan Ma, Liang-Gee Chen, National Taiwan University, Taiwan

DISPS-P1.6: CLOSED-FORM APPROXIMATION OF LINEAR PHASE IIR DIGITAL FILTERS 1645

WITH GUARANTEED STABILITY

Masayoshi Nakamoto, Shuichi Ohno, Hiroshima University, Japan

DISPS-P1.7: A DESIGN PROCEDURE FOR OVERSAMPLED NONUNIFORM FILTER 1649 BANKS WITH PERFECT-RECONSTRUCTION

Mohamed Mansour, Texas Instruments Inc., United States

DISPS-P1.8: REAL-TIME DESIGN OF A SPACE/SPATIAL-FREQUENCY OPTIMAL FILTER 1653 FOR HIGHLY NONSTATIONARY TWO-DIMENSIONAL SIGNAL ESTIMATION

Veselin Ivanovic, Nevena Radovic, Srdjan Jovanovski, University of Montenegro, Montenegro

DISPS-P1.9: THE LD-RLS ALGORITHM WITH DIRECTIONAL FORGETTING 1657 IMPLEMENTED ON A VECTOR-LIKE HARDWARE ACCELERATOR

Roman Bartosinski, Institute of Information Theory and Automation / UTIA AV CR, Czech Republic

DISPS-P1.10: LEAST SQUARES APPROXIMATION AND POLYPHASE DECOMPOSITION 1661 FOR PIPELINING RECURSIVE FILTERS

Aditya Gupta, Andrew Singer, Naresh Shanbhag, University of Illinois Urbana-Champaign, United States

\section{DISPS-P2: ERROR CORRECTION CODING AND COMMUNICATION SYSTEM DESIGN}

DISPS-P2.1: HARDWARE ARCHITECTURES FOR SUCCESSIVE CANCELLATION 1665 DECODING OF POLAR CODES

Camille Leroux, McGill University, Canada; Ido Tal, Alexander Vardy, University of California San Diego, United States; Warren J. Gross, McGill University, Canada

DISPS-P2.2: RECONFIGURABLE DECODER ARCHITECTURES FOR RAPTOR CODES 1669

Hady Zeineddine, Mohammad Mansour, American University of Beirut, Lebanon

DISPS-P2.3: A FLEXIBLE HIGH-THROUGHPUT HARDWARE ARCHITECTURE FOR A 1673 GAUSSIAN NOISE GENERATOR

Ioannis Paraskevakos, Vassilis Paliouras, University of Patras, Greece

DISPS-P2.4: LATENCY-CONSTRAINED LOW-COMPLEXITY LATTICE REDUCTION FOR 1677 MIMO-OFDM SYSTEMS

Chun-Fu Liao, Fang-Chun Lan, National Tsing Hua University, Taiwan; Po-lin Chiu, National Chiao Tung University, Taiwan; Yuan-Hao Huang, National Tsing Hua University, Taiwan

DISPS-P2.5: IMPROVING THE PERFORMANCE OF DSP SYSTEMS FOR MIMO 1681 PROCESSING

Nathaniel Horner, Intel Corporation, United States; Andres Kwasinski, Antonio Mondragon, Rochester Institute of Technology, United States

DISPS-P2.6: REAL-TIME DVB-S2 LDPC DECODING ON MANY-CORE GPU 1685 ACCELERATORS

Gabriel Falcao, Instituto de Telecomunicações, Portugal; Joao Andrade, Instituto de Telecomunicações / University of Porto, Portugal; Vitor Silva, Instituto de Telecomunicações, Portugal; Leonel Sousa, INESC-ID, Portugal FOR VECTOR PRECODING

Maitane Barrenechea, University of Mondragon, Spain; Luis Barbero, Queen's University Belfast, United Kingdom; Idoia Jiménez, Egoitz, Arruti, Mikel Mendicute, University of Mondragon, Spain 


\section{DISPS-P3: IMPLEMENTATION TECHNIQUES FOR AUDIO, VIDEO, AND FFTS}

DISPS-P3.1: HARDWARE ACCELERATION OF ITERATIVE IMAGE RECONSTRUCTION FOR 1697 X-RAY COMPUTED TOMOGRAPHY

Jung Kuk Kim, Zhengya Zhang, Jeffrey A. Fessler, University of Michigan, United States

DISPS-P3.2: ENERGY-OPTIMIZED HIGH PERFORMANCE FFT PROCESSOR

Dongsuk Jeon, Mingoo Seok, University of Michigan, United States; Chaitali Chakrabarti, Arizona State University, United

States; David Blaauw, Dennis Sylvester, University of Michigan, United States

DISPS-P3.3: A FPGA ARCHITECTURE FOR REAL-TIME PROCESSING OF 1705 VARIABLE-LENGTH FFTS

Stefan Langemeyer, Peter Pirsch, Holger Blume, Leibniz University Hannover, Germany

DISPS-P3.4: CONFLICT-FREE PARALLEL ACCESS SCHEME FOR MIXED_RADIX FFT 1709 SUPPORTING I/O PERMUTATIONS

Harri Sorokin, Jarmo Takala, Tampere University of Technology, Finland

DISPS-P3.5: FAST PHYSICAL OBJECT IDENTIFICATION BASED ON UNCLONABLE 1713 FEATURES AND SOFT FINGERPRINTING

Taras Holotyak, Sviatoslav Voloshynovskiy, Oleksiy Koval, Fokko Beekhof, University of Geneva, Switzerland

DISPS-P3.6: EXPLOITING RECONFIGURABLE SWP OPERATORS FOR MULTIMEDIA 1717 APPLICATIONS

Daniel Menard, Hai-Nam Nguyen, University of Rennes, France; Fracois Charot, INRIA, France; Stephane Guyetant, CEA, France; Jeremie Guillot, LIRMM, France; Erwan Raffin, Emmanuel Casseau, University of Rennes, France

DISPS-P3.8: THE CENTERED DISCRETE FOURIER TRANSFORM AND A PARALLEL 1725 IMPLEMENTATION OF THE FFT

Dale Mugler, The University of Akron, United States

DISPS-P3.9: A HIGH THROUGHPUT PARALLEL AVC/H.264 CONTEXT-BASED ADAPTIVE 1729 BINARY ARITHMETIC DECODER Jia-Wei Liang, He-Yuan Lin, Gwo Giun Lee, National Cheng Kung University, Taiwan

DISPS-P3.10: H- AND C-LEVEL WFST-BASED LARGE VOCABULARY CONTINUOUS 1733 SPEECH RECOGNITION ON GRAPHICS PROCESSING UNIS

Jungsuk Kim, Seoul National University, Republic of Korea; Kisun You, Qualcomm Korea Ltd., Republic of Korea; Wonyong Sung, Seoul National University, Republic of Korea

\section{ITT-L1: INDUSTRIAL TECHNOLOGY FOR SPEECH PROCESSING APPLICATIONS}

ITT-L1.1: BINAURAL EXTENSION AND PERFORMANCE OF SINGLE-CHANNEL SPECTRAL 1737 SUBTRACTION DEREVERBERATION ALGORITHMS

Alexandros Tsilfidis, Eleftheria Georganti, John Mourjopoulos, University of Patras, Greece 
Muhammad Ikram, Texas Instruments Inc., United States

ITT-L1.3: TWO-CHANNEL POST-FILTERING BASED ON ADAPTIVE SMOOTHING AND 1745 NOISE PROPERTIES

Chengshi Zheng, Yi Zhou, Xiaohu Hu, Xiaodong Li, Institute of Acoustics Chinese Academy of Sciences, China

ITT-L1.4: SPEECH PROCESSING AND RETRIEVAL IN A PERSONAL MEMORY AID 1749 SYSTEM FOR THE ELDERLY

Alexander Sorin, Hagai Aronowitz, Jonathan Mamou, Orith Toledo-Ronen, Ron Hoory, Michael Kuritzky, Yael Erez, IBM Haifa Research Lab, Israel; Bhuvana Ramabhadran, Abhinav Sethy, IBM T.J. Watson Research Center, United States

ITT-L1.5: CROVER: IMPROVING ROVER USING AUTOMATIC ERROR DETECTION............................................. 1753

Kacem Abida, Fakhri Karray, University of Waterloo, Canada; Wafa Abida, Vestec, Canada

ITT-L1.6: TEMPLATE-BASED METHODS FOR SENTENCE GENERATION AND SPEECH 1757 SYNTHESIS

Hiroyuki Segi, Reiko Takou, Nobumasa Seiyama, Japan Broadcasting Corporation, Japan; Tohru Takagi, NHK Engineering Services Inc., Japan; Hideo Saito, Keio University, Japan; Shinji Ozawa, Aichi University of Technology, Japan

\section{ITT-P1: INDUSTRIAL TECHNOLOGY FOR DEFENSE, COMMUNICATION, AND OTHER} APPLICATIONS

ITT-P1.1: HORIZONTAL SMALL TARGET DETECTION WITH COOPERATIVE 1761 BACKGROUND ESTIMATION AND REMOVAL FILTERS

Sungho Kim, Yeungnam University, Republic of Korea; Yukyung Yang, Joohyoung Lee, Agency for Defense Development, Republic of Korea

ITT-P1.2: DETECTION AND SEGMENTATION OF FMCW RADAR SIGNALS BASED ON THE 1765 CHIRPLET TRANSFORM

Fabien Millioz, Michael Davies, University of Edinburgh, United Kingdom

ITT-P1.3: BLIND PHASE RECOVERY IN QAM COMMUNICATION SYSTEMS USING 1769 CHARACTERISTIC FUNCTION

Ehsan Hassani Sadi, Hamidreza Amindavar, Amirkabir University of Technology, Iran

ITT-P1.4: AN IMPROVEMENT ON GM-PHD FILTER FOR OCCLUDED TARGET 1773 TRACKING

Mahdi Yazdian Dehkordi, Zohreh Azimifar, Mohammad Ali Masnadi-Shirazi, Shiraz University, Iran

ITT-P1.5: A RADIO FREQUENCY IDENTIFICATION SYSTEM FOR ACCURATE INDOOR 1777 LOCALIZATION

Akshay Athalye, CEWIT / Stony Brook University, United States; Vladimir Savic, Universidad Politecnica de Madrid, Spain;

Miodrag Bolic, University of Ottawa, Canada; Petar Djuric, Stony Brook University, United States

ITT-P1.6: REAL-LIFE SPEECH-ENABLED SYSTEM TO ENHANCE INTERACTION WITH 1781 RFID NETWORKS IN NOISY ENVIRONMENTS

Yacine Benahmed, INRS-EMT, Canada; Sid-Ahmed Selouani, Université de Moncton, Canada; Douglas O'Shaughnessy, INRS-

EMT, Canada; Amin Haji Abolhassani, McGill University, Canada

ITT-P1.7: FRAME ERROR-ROBUST MDCT BIT REDUCTION SCHEME USING 1785 INTER-FRAME CORRELATION FOR G.729.1

Keunseok Cho, Korea Advanced Institute of Science and Technology, Republic of Korea; Sangbae Jung, Gyeongsang National University, Republic of Korea; Hyunwoo Kim, ETRI, Republic of Korea; Minsoo Hahn, Korea Advanced Institute of Science and Technology, Republic of Korea 

COMPOSITES USING ULTRASONIC SIGNALS

Nicolas Bochud, Ángel M. Gómez, Guillermo Rus, University of Granada, Spain; José Luis Carmona Maqueda, Antonio Miguel Peinado Herreros, Universidad de Granada, Spain

ITT-P1.9: INDUSTRIAL APPLICATION OF ACOUSTO-ULTRASONIC SIGNAL QUALITY AND 1793 ROBUST TIME-OF-FLIGHT ESTIMATION FOR ANISOTROPIC MATERIALS

Ladislav Jerabek, Anthony Bartos, Jan Strycek, Airstar Inc., United States

ITT-P1.10: A NEW APPROACH FOR THE ESTIMATION OF THE POROSITY IN NMR.

Fred Gruber, Lalitha Venkataramanan, Denise Freed, Tarek Habashy, Schlumberger-Doll Research Center, United States

ITT-P1.11: REDUCTION OF MULTIPLE HARMONICS EM NOISE IN HELICOPTER 1801 COCKPIT

Ari Abramson, Ilan Efrat, Elbit Systems, Israel

\section{ITT-P2: INDUSTRIAL TECHNOLOGY FOR IMAGING, AUTOMOTIVE, AND BIOMEDICAL APPLICATIONS}

ITT-P2.1: BOOSTING VIDEO CLASSIFICATION USING CROSS-VIDEO SIGNALS. 1805

Mehmet Emre Sargin, Hrishikesh Aradhye, Google Inc., United States

ITT-P2.2: SLICE ERROR CONCEALMENT BASED ON SIZE-ADAPTIVE SSIM MATCHING 1809 AND MOTION VECTOR OUTLIER REJECTION

Hai Gao, Jo Yew Tham, Wei Siong Lee, Kwong Huang Goh, Institute for Infocomm Research / Agency of Science Technology And Research, Singapore

ITT-P2.3: A MEDIA MONITORING SOLUTION 1813

João Neto, Hugo Meinedo, INESC-ID/IST, Portugal; Márcio Viveiros, VoiceInteraction, Portugal

ITT-P2.4: FLAME DETECTION METHOD IN VIDEO USING COVARIANCE DESCRIPTORS 1817

Yusuf Hakan Habiboglu, Turkish Navy, Turkey; Osman Günay, A. Enis Çetin, Bilkent University, Turkey

ITT-P2.5: COLOR CORRECTION FOR OBJECT TRACKING ACROSS MULTIPLE CAMERAS 1821

Satyam Srivastava, Ka Ki Ng, Edward Delp, Purdue University, United States

ITT-P2.6: GEAR SCALE ESTIMATION FOR SYNTHETIC SPEED PULSE GENERATION

John-Olof Nilsson, Isaac Skog, Alessio De Angelis, Claudia Aquilanti, Peter Händel, KTH - Royal Institute of Technology,

Sweden

ITT-P2.7: DRIVER RISK EVALUATION BASED ON ACCELERATION, DECELERATION, AND 1829 STEERING BEHAVIOR

Chiyomi Miyajima, Hiroki Ukai, Atsumi Naito, Hideomi Amata, Norihide Kitaoka, Kazuya Takeda, Nagoya University, Japan

ITT-P2.8: FALL DETECTION IN A SMART ROOM BY USING A FUZZY ONE CLASS 1833 SUPPORT VECTOR MACHINE AND IMPERFECT TRAINING DATA

Miao Yu, Syed Mohsen Naqvi, Adel Rhuma, Jonathon A. Chambers, Loughborough University, United Kingdom

ITT-P2.9: MONITORING WORKSPACE ACTIVITIES USING ACCELEROMETERS 1837

Natali Ruchansky, Boston University, United States; Claire Lochner, University at Buffalo, United States; Elizabeth Do, Smith College, United States; Tremaine Rawls, Norfolk State University, United States; Nabil Hajj Chehade, Jay Chien, Greg Pottie, William Kaiser, University of California Los Angeles, United States

\section{IFS-L1: WATERMARKING AND MULTIMEDIA SECURITY}


Patrick Bas, Lagis - Centre National de la Recherche Scientifique, France

IFS-L1.4: TOWARDS IMPROVING NETWORK FLOW WATERMARKS USING THE 1852 REPEAT-ACCUMULATE CODES

Amir Houmansadr, Nikita Borisov, University of Illinois Urbana-Champaign, United States

IFS-L1.5: EQUIANGULAR TIGHT FRAME FINGERPRINTING CODES 1856

Dustin Mixon, Princeton University, United States; Christopher Quinn, Negar Kiyavash, University of Illinois Urbana-

Champaign, United States; Matthew Fickus, Air Force Institute of Technology, United States

IFS-L1.6: MODELING TEMPORAL CORRELATIONS IN CONTENT FINGERPRINTS 1860

Avinash Varna, Min Wu, University of Maryland College Park, United States

\section{IFS-P1: INFORMATION FORENSICS AND NETWORK SECURITY}

IFS-P1.1: SECURITY OF COPY-MOVE FORGERY DETECTION TECHNIQUES 1864

Hieu Cuong Nguyen, Stefan Katzenbeisser, Darmstadt University of Technology, Germany

IFS-P1.2: DIFFERENTIATING BETWEEN COMPUTER GENERATED AND NATURAL 1868 IMAGES USING WAVELET BASED TRANSFORMS

Levent Özparlak, Ismail Avcibas, Baskent University, Turkey

IFS-P1.3: ON THE EFFECT OF AMR AND AMR-WB GSM COMPRESSION ON 1872 OVERLAPPED SPEECH FOR FORENSIC ANALYSIS

Eva Cheng, Ian S. Burnett, RMIT University, Australia

IFS-P1.4: ANTI-FORENSICS FOR FRAME DELETION/ADDITION IN MPEG VIDEO 1876

Matthew Stamm, K. J. Ray Liu, University of Maryland College Park, United States

IFS-P1.5: EXPOSING DUPLICATED REGIONS AFFECTED BY REFLECTION, ROTATION 1880 AND SCALING

Sergio Bravo-Solorio, Asoke Nandi, The University of Liverpool, United Kingdom

IFS-P1.6: THE COST OF JPEG COMPRESSION ANTI-FORENSICS 1884

Giuseppe Valenzise, Marco Tagliasacchi, Stefano Tubaro, Politecnico di Milano, Italy

IFS-P1.7: BAYESIAN TOPIC MODELS FOR DESCRIBING COMPUTER NETWORK 1888 BEHAVIORS

Christopher Cramer, Signal Innovations Group Inc., United States; Lawrence Carin, Duke University, United States

IFS-P1.9: VULNERABILITY OF INSENS TO DENIAL OF SERVICE ATTACKS 1896

Kashif Saghar, David Kendall, Ahmed Bouridane, Northumbria University Newcastle, United Kingdom

IFS-P1.10: SECRECY CAPACITY AND SECURE OUTAGE PERFORMANCE FOR RAYLEIGH 1900 FADING SIMO CHANNEL

Md. Zahurul I. Sarkar, Tharmalingam Ratnarajah, Queen's University Belfast, United Kingdom

IFS-P1.11: A ROBUST QUANTIZATION METHOD USING A ROBUST CHINESE 1904 REMAINDER THEOREM FOR SECRET KEY GENERATION

Wenjie Wang, Chen Wang, Ministry of Education Key Lab for Intelligent Networks and Network Security, China; Xiang-Gen Xia, University of Delaware, United States 
IFS-P2.1: SPEAKER AUTHENTICATION USING VIDEO-BASED LIP INFORMATION

Budhaditya Goswami, Chi Ho Chan, Josef Kittler, William Christmas, University of Surrey, United Kingdom

IFS-P2.2: AUTHENTICATION OF FINGERPRINT SCANNERS

1912

Vladimir Ivanov, John Baras, University of Maryland College Park, United States

IFS-P2.3: ECG FOR BLIND IDENTITY VERIFICATION IN DISTRIBUTED SYSTEMS 1916

Jiexin Gao, Foteini Agrafioti, Hoda Mohammadzade, Dimitrios Hatzinakos, University of Toronto, Canada

IFS-P2.4: ZERO LEAKAGE QUANTIZATION SCHEME FOR BIOMETRIC VERIFICATION

Joep de Groot, Jean-Paul Linnartz, Eindhoven University of Technology, Netherlands

IFS-P2.5: A COMPARATIVE ANALYSIS OF BIOMETRIC SECRET-KEY BINDING SCHEMES 1924 BASED ON QIM AND WYNER-ZIV CODING

Aniketh Talwai, Indian Institute of Technology Guwahati, India; Francis M. Bui, Ashish Khisti, Dimitrios Hatzinakos, University of Toronto, Canada

IFS-P2.6: A KNOWLEDGE-BASED ALGORITHM TO REMOVE BLOCKING ARTIFACTS IN 1928 SKIN IMAGES FOR FORENSIC ANALYSIS

Chaoying Tang, Adams Wai Kin Kong, Nanyang Technological University, Singapore; Noah Craft, Harbor-UCLA Medical Center, United States

IFS-P2.7: SMART METER PRIVACY USING A RECHARGEABLE BATTERY: MINIMIZING 1932 THE RATE OF INFORMATION LEAKAGE

David Varodayan, Hewlett-Packard Laboratories, United States; Ashish Khisti, University of Toronto, Canada

IFS-P2.8: ONLINE ANOMALY DETECTION WITH EXPERT SYSTEM FEEDBACK IN 1936 SOCIAL NETWORKS

Corinne Horn, Rebecca Willett, Duke University, United States

IFS-P2.9: ACOUSTIC DETECTION AND CLASSIFICATION USING TEMPORAL AND 1940 FREQUENCY MULTIPLE ENERGY DETECTOR FEATURES

Jorge Moragues, Arturo Serrano, Luis Vergara, Jorge Gosalbez, Polytechnic University of Valencia, Spain

IFS-P2.10: ABNORMAL MOTION DETECTION IN CROWDED SCENES USING LOCAL 1944 SPATIO-TEMPORAL ANALYSIS

Fahad Daniyal, Andrea Cavallaro, Queen Mary University of London, United Kingdom

IFS-P2.11: A 3-LAYER CODING SCHEME FOR BIOMETRY TEMPLATE PROTECTION 1948 BASED ON SPECTRAL MINUTIAE

Xiaoying Shao, Haiyun Xu, Raymond N.J. Veldhuis, Cornelis H. Slump, University of Twente, Netherlands

\section{MLSP-L1: LEARNING THEORY AND MODELS I}

MLSP-L1.1: USPACOR: UNIVERSAL SPARSITY-CONTROLLING OUTLIER REJECTION.

Georgios B. Giannakis, Gonzalo Mateos, Shahrokh Farahmand, Vassilis Kekatos, Hao Zhu, University of Minnesota, United States

MLSP-L1.2: VARIABILITY REGULARIZATION IN LARGE-MARGIN CLASSIFICATION 1956

Dwi Sianto Mansjur, Ted S. Wada, Biing-Hwang(Fred) Juang, Georgia Institute of Technology, United States

MLSP-L1.3: SPARSE GRAPHICAL MODELING OF PIECEWISE-STATIONARY TIME SERIES. 1960

Daniele Angelosante, Georgios B. Giannakis, University of Minnesota, United States

MLSP-L1.4: FACTOR GRAPH-BASED STRUCTURAL EQUILIBRIA IN DYNAMICAL GAMES

Liming Wang, University of Illinois Chicago, United States; Vikram Krishnamurthy, University of British Columbia, Canada; Dan Schonfeld, University of Illinois Chicago, United States 
Haoyang Liu, Keqin Liu, Qing Zhao, University of California Davis, United States

MLSP-L1.6: INFINITE-STATE SPECTRUM MODEL FOR MUSIC SIGNAL ANALYSIS. 1972

Masahiro Nakano, The University of Tokyo, Japan; Jonathan Le Roux, Hirokazu Kameoka, NTT Communication Science

Laboratories, Japan; Nobutaka Ono, Shigeki Sagayama, The University of Tokyo, Japan

\section{MLSP-L2: NON-NEGATIVE TENSOR FACTORIZATION AND BLIND SEPARATION}

MLSP-L2.1: MULTIPLE KERNEL NONNEGATIVE MATRIX FACTORIZATION

Shounan An, LG Electronics, Republic of Korea; Jeong-Min Yun, Seungjin Choi, POSTECH, Republic of Korea

MLSP-L2.2: MAJORIZATION-MINIMIZATION ALGORITHM FOR SMOOTH ITAKURA-SAITO 1980 NONNEGATIVE MATRIX FACTORIZATION

Cédric Févotte, CNRS LTCI / Télécom ParisTech, France

MLSP-L2.3: NOVEL HIERARCHICAL ALS ALGORITHM FOR NONNEGATIVE TENSOR 1984 FACTORIZATION

Anh-Huy Phan, Andrzej Cichocki, Brain Science Institue, Japan; Kiyotoshi Matsuoka, Kyushu University of Technology, Japan; Jianting Cao, Brain Science Institue, Japan

MLSP-L2.4: FAST DAMPED GAUSS-NEWTON ALGORITHM FOR SPARSE AND 1988 NONNEGATIVE TENSOR FACTORIZATION

Anh-Huy Phan, Brain Science Institue, Japan; Petr Tichavský, Institute of Information Theory and Automation, Czech Republic; Andrzej Cichocki, Brain Science Institue, Japan

MLSP-L2.5: MAXIMUM MARGINAL LIKELIHOOD ESTIMATION FOR NONNEGATIVE 1992 DICTIONARY LEARNING

Onur Dikmen, Cédric Févotte, CNRS LTCI / Télécom ParisTech, France

MLSP-L2.6: A SPARSITY BASED CRITERION FOR SOLVING THE PERMUTATION 1996 AMBIGUITY IN CONVOLUTIVE BLIND SOURCE SEPARATION

Radoslaw Mazur, Alfred Mertins, University of Luebeck, Germany

\section{MLSP-L3: MACHINE LEARNING METHODS AND APPLICATIONS III}

MLSP-L3.1: GEOMETRIC PROGRAMMING FOR AGGREGATION OF BINARY 2000 CLASSIFIERS

Sunho Park, Seungjin Choi, POSTECH, Republic of Korea

MLSP-L3.2: A LEARNING-BASED APPROACH TO EXPLOSIVES DETECTION USING 2004 MULTI-ENERGY X-RAY COMPUTED TOMOGRAPHY

Limor Eger, Boston University, United States; Synho Do, Massachusetts General Hospital, United States; Prakash Ishwar, William Clem Karl, Boston University, United States; Homer Pien, Massachusetts General Hospital, United States

MLSP-L3.3: ENTROPY ESTIMATION USING THE PRINCIPLE OF MAXIMUM ENTROPY 2008 Behrouz Behmardi, Raviv Raich, Oregon State University, United States; Alfred O. Hero III, University of Michigan, United States

MLSP-L3.4: TIME-FREQUENCY SEGMENTATION OF BIRD SONG IN NOISY ACOUSTIC 2012 ENVIRONMENTS

Lawrence Neal, Forrest Briggs, Raviv Raich, Xiaoli Fern, Oregon State University, United States

MLSP-L3.5: ESTIMATION OF SYMMETRIC CHI-SQUARE DIVERGENCE FOR POINT 2016 PROCESSES

Il Park, Sohan Seth, Murali Rao, Jose C. Principe, University of Florida, United States 


\section{MLSP-P1: MACHINE LEARNING METHODS AND APPLICATIONS I}

MLSP-P1.1: FINDING CURVES IN SAR CCD IMAGES

Miriam Cha, Rhonda D. Phillips, Michael Yee, MIT Lincoln Laboratory, United States

MLSP-P1.2: A METHOD TO INFER EMOTIONS FROM FACIAL ACTION UNITS

Sudha Velusamy, Hariprasad Kannan, Balasubramanian Anand, Anshul Sharma, Bilva Navathe, Samsung India Software

Operations Pvt Ldt, India

MLSP-P1.4: A NEW METHOD FOR VISUAL STYLOMETRY ON IMPRESSIONIST PAINTINGS

Hanchao Qi, Shannon Hughes, University of Colorado at Boulder, United States

MLSP-P1.5: ONLINE KERNEL SVM FOR REAL-TIME FMRI BRAIN STATE PREDICTION 2040

Yongxin Xi, Hao Xu, Ray Lee, Peter Ramadge, Princeton University, United States

MLSP-P1.6: ON VISUALLY EVOKED POTENTIALS IN EEG INDUCED BY MULTIPLE PSEUDORANDOM BINARY SEQUENCES FOR BRAIN COMPUTER INTERFACE DESIGN

Hooman Nezamfar, Umut Orhan, Deniz Erdogmus, Northeastern University, United States; Kenneth Hild, Oregon Health and Science University, United States; Shalini Purwar, Northeastern University, United States; Barry Oken, Melanie Fried-Oken, Oregon Health and Science University, United States

MLSP-P1.7: TRADING OFF COMMUNICATIONS BANDWIDTH WITH ACCURACY IN 2048 ADAPTIVE DIFFUSION NETWORKS

Symeon Chouvardas, University of Athens, Greece; Konstantinos Slavakis, University of Peloponnese, Greece; Sergios

Theodoridis, University of Athens, Greece

MLSP-P1.8: FEATURE SELECTION THROUGH GRAVITATIONAL SEARCH ALGORITHM 2052

Joao Papa, Andre Pagnin, Silvana Artioli Schellini, Universidade Estadual Paulista, Brazil; Andre Spadotto, Rodrigo Guido, Moacir Ponti, University of Sao Paulo, Brazil; Giovani Chiachia, Alexandre Falcao, University of Campinas, Brazil

MLSP-P1.9: PREDICTIVE MODELING OF THE SPATIOTEMPORAL EVOLUTION OF AN 2056 ENVIRONMENTAL HAZARD AND ITS SENSOR NETWORK IMPLEMENTATION

Dimitris V. Manatakis, Elias S. Manolakos, University of Athens, Greece

MLSP-P1.10: DENOISING SPARSE NOISE VIA ONLINE DICTIONARY LEARNING.

Anoop Cherian, University of Minnesota Twin Cities, United States; Suvrit Sra, Max Planck Institute for Biological Cybernetics, Germany; Nikolaos Papanikolopoulos, University of Minnesota Twin Cities, United States

MLSP-P1.11: CO-CLUSTERING AS MULTILINEAR DECOMPOSITION WITH SPARSE 2064 LATENT FACTORS

Evagelos Papalexakis, Nikolaos Sidiropoulos, Technical University of Crete, Greece

\section{MLSP-P2: KERNEL MODELS AND MACHINE LEARNING APPLICATIONS}

MLSP-P2.1: A KERNELIZED MAXIMAL-FIGURE-OF-MERIT LEARNING APPROACH BASED 2068 ON SUBSPACE DISTANCE MINIMIZATION

Byungki Byun, Chin-Hui Lee, Georgia Institute of Technology, United States 
CLASSIFIERS

Franz Pernkopf, Michael Wohlmayr, Graz University of Technology, Austria; Manfred Mücke, University of Vienna, Austria

MLSP-P2.4: FINDING DEPENDENCIES BETWEEN FREQUENCIES WITH THE KERNEL CROSS-SPECTRAL DENSITY

Michel Besserve, Dominik Janzing, Nikos K. Logothetis, Bernhard Schölkopf, MPI for Biological Cybernetics, Germany

MLSP-P2.5: LO SPARSE GRAPHICAL MODELING

Goran Marjanovic, Victor Solo, University of New South Wales, Australia

MLSP-P2.6: A SEGMENTATION METHOD FOR TEXTURED IMAGES BASED ON THE

MAXIMUM POSTERIOR MODE CRITERION

Frederic Lehmann, Telecom SudParis, France

MLSP-P2.7: SPHERE PACKING FOR CLUSTERING SETS OF VECTORS IN FEATURE

SPACE

Darío García-García, Raúl Santos-Rodríguez, Universidad Carlos III de Madrid, Spain

MLSP-P2.8: POLYTOPE KERNEL DENSITY ESTIMATES ON DELAUNAY GRAPHS

Erhan Bas, Deniz Erdogmus, Northeastern University, United States

MLSP-P2.9: NON-FLAT CLUSTERING WITH ALPHA-DIVERGENCES

Olivier Schwander, Frank Nielsen, École Polytechnique, France

MLSP-P2.10: A METRIC APPROACH TOWARD POINT PROCESS DIVERGENCE

Sohan Seth, Austin Brockmeier, Jose C. Principe, University of Florida, United States

\section{MLSP-P3: MACHINE LEARNING METHODS AND APPLICATIONS II}

MLSP-P3.1: BLIND SEPARATION OF MULTIPLE BINARY SOURCES FROM ONE

NONLINEAR MIXTURE

Konstantinos Diamantaras, TEI of Thessaloniki, Greece; Theophilos Papadimitriou, Democritus University of Thrace, Greece;

Gabriela Vranou, TEI of Thessaloniki, Greece

MLSP-P3.2: BLIND BEAMFORMER FOR CONSTANT MODULUS SIGNALS BASED ON 2112 RELEVANCE VECTOR MACHINE

Kyuho Hwang, Sooyong Choi, Yonsei University, Republic of Korea

MLSP-P3.3: NATURAL GRADIENT APPROACH IN ORTHOGONAL MATRIX OPTIMIZATION 2116 USING CAYLEY TRANSFORM

Gen Hori, Asia University / RIKEN, Japan

MLSP-P3.4: NONSTATIONARY AND TEMPORALLY CORRELATED SOURCE SEPARATION USING GAUSSIAN PROCESS

Hsin-Lung Hsieh, Jen-Tzung Chien, National Cheng Kung University, Taiwan

MLSP-P3.5: ONLINE FEATURE SELECTION AND CLASSIFICATION

Habil Kalkan, Bayram Cetisli, Suleyman Demirel University, Turkey

MLSP-P3.6: A SLIDING-WINDOW ONLINE FAST VARIATIONAL SPARSE BAYESIAN LEARNING ALGORITHM

Thomas Buchgraber, Graz University of Technology, Austria; Dmitriy Shutin, H. Vincent Poor, Princeton University, United States 
MLSP-P3.7: ADAPTIVE MODELLING WITH TUNABLE RBF NETWORK USING

MULTI-INNOVATION RLS ALGORITHM ASSISTED BY SWARM INTELLIGENCE

Hao Chen, Yu Gong, Xia Hong, University of Reading, United Kingdom

MLSP-P3.8: SEMI-SUPERVISED HANDWRITTEN DIGIT RECOGNITION USING VERY 2136

FEW LABELED DATA

Steven Van Vaerenbergh, Ignacio Santamaría, University of Cantabria, Spain; Paolo Emilio Barbano, University of Cambridge,

United Kingdom

MLSP-P3.9: A REGULARIZATION FRAMEWORK FOR MOBILE SOCIAL NETWORK 2140 ANALYSIS

Xiaowen Dong, Pascal Frossard, Pierre Vandergheynst, Ecole Polytechnique Fédérale de Lausanne, Switzerland; Nikolai

Nefedov, Nokia Research Center (Lausanne), Switzerland

MLSP-P3.10: NON-LINEAR TAGGING MODELS WITH LOCALIST AND DISTRIBUTED 2144 WORD REPRESENTATIONS

Sumit Chopra, Srinivas Bangalore, AT\&T Labs Research, United States

\section{MLSP-P4: LEARNING THEORY AND MODELS II}

MLSP-P4.1: STABILITY ANALYSIS OF MULTIPLICATIVE UPDATE ALGORITHMS FOR 2148 NON-NEGATIVE MATRIX FACTORIZATION

Roland Badeau, Télécom ParisTech / CNRS LTCI, France; Nancy Bertin, Emmanuel Vincent, INRIA, France

MLSP-P4.2: A MACHINE LEARNING BASED APPROACH TO WEATHER PARAMETER 2152 ESTIMATION IN DOPPLER WEATHER RADAR

Satoshi Kon, Toshihisa Tanaka, Tokyo University of Agriculture and Technology, Japan; Humihiko Mizutani, Masakazu Wada, Social Infrastructure System Company / Toshiba Corporation, Japan

MLSP-P4.3: BAYESIAN REINFORCEMENT LEARNING FOR POMDP-BASED DIALOGUE 2156 SYSTEMS

ShaoWei Png, Joelle Pineau, McGill University, Canada

MLSP-P4.4: SPARSE CODING AND DICTIONARY LEARNING BASED ON THE MDL 2160 PRINCIPLE

Ignacio Ramirez, Guillermo Sapiro, University of Minnesota, United States

MLSP-P4.5: SIMILARITY LEARNING FOR SEMI-SUPERVISED MULTI-CLASS BOOSTING

Q Y Wang, Pong C Yuen, Baptist University, Hong Kong SAR of China; G C Feng, Sun Yat-Sen University, China

MLSP-P4.6: JOINT DICTIONARY LEARNING AND TOPIC MODELING FOR IMAGE 2168 CLUSTERING

Lingbo Li, Mingyuan Zhou, Eric Wang, Lawrence Carin, Duke University, United States

MLSP-P4.8: AN EFFICIENT RANK-DEFICIENT COMPUTATION OF THE PRINCIPLE OF 2176 RELEVANT INFORMATION

Luis Sanchez Giraldo, Jose C. Principe, University of Florida, United States

MLSP-P4.9: FAST ADAPTIVE VARIATIONAL SPARSE BAYESIAN LEARNING WITH 2180 AUTOMATIC RELEVANCE DETERMINATION

Dmitriy Shutin, Princeton University, United States; Thomas Buchgraber, Graz University of Technology, Austria; Sanjeev R. Kulkarni, H. Vincent Poor, Princeton University, United States

MLSP-P4.10: TIME-EVOLVING MODELING OF SOCIAL NETWORKS 2184

Eric Wang, Jorge Silva, Rebecca Willett, Lawrence Carin, Duke University, United States 


\section{LEARNING}

Sevket Derin Babacan, University of Illinois Urbana-Champaign, United States; Martin Luessi, Northwestern University, United States; Rafael Molina, Universidad de Granada, Spain; Aggelos K. Katsaggelos, Northwestern University, United States

\section{MLSP-P5: MACHINE LEARNING FOR SPEECH AND AUDIO APPLICATIONS}

MLSP-P5.1: AUDIO SOURCE SEPARATION BY BASIS FUNCTION ADAPTATION 2192

Yinyi Guo, Mofei Zhu, Stanford University, United States

MLSP-P5.2: COMBINING MONAURAL SOURCE SEPARATION WITH LONG 2196 SHORT-TERM MEMORY FOR INCREASED ROBUSTNESS IN VOCALIST GENDER RECOGNITION

Felix Weninger, Technische Universität München, Germany; Jean-Louis Durrieu, Ecole Polytechnique Fédérale de Lausanne, Switzerland; Florian Eyben, Technische Universität München, Germany; Gaël Richard, Télécom ParisTech / LTCI-CNRS, France; Björn Schuller, Technische Universität München, Germany

MLSP-P5.3: LEARNING VOCAL TRACT VARIABLES WITH MULTI-TASK KERNELS 2200 Hachem Kadri, INRIA Lille, France; Emmanuel Duflos, Ecole Centrale de Lille, France; Philippe Preux, INRIA Lille, France

MLSP-P5.4: SPEAKER RECOGNITION USING MULTIPLE KERNEL LEARNING BASED 2204 ON CONDITIONAL ENTROPY MINIMIZATION

Tetsuji Ogawa, Hideitsu Hino, Waseda University, Japan; Nima Reyhani, Aalto University, Finland; Noboru Murata, Tetsunori Kobayashi, Waseda University, Japan

MLSP-P5.5: EXPLOITING ACTIVE-LEARNING STRATEGIES FOR ANNOTATING 2208 PROSODIC EVENTS WITH LIMITED LABELED DATA

Raul Fernandez, Bhuvana Ramabhadran, IBM Research, United States

MLSP-P5.6: THE RWTH 2010 QUAERO ASR EVALUATION SYSTEM FOR ENGLISH, 2212 FRENCH, AND GERMAN

Martin Sundermeyer, Markus Nussbaum-Thom, Simon Wiesler, Christian Plahl, Amr El-Desoky Mousa, Stefan Hahn, David Nolden, Ralf Schlüter, Hermann Ney, RWTH Aachen University, Germany

MLSP-P5.7: HIERARCHICAL AUDIO CLASSIFICATION USING CEPSTRAL MODULATION 2216 RATIO REGRESSIONS BASED ON LEGENDRE POLYNOMIALS

Anil Nagathil, Peter Göttel, Rainer Martin, Ruhr-Universität Bochum, Germany

MLSP-P5.8: TRANSIENT ACOUSTIC SIGNAL CLASSIFICATION USING JOINT SPARSE 2220 REPRESENTATION

Haichao Zhang, University of Illinois Urbana-Champaign / Northwestern Polytechnical University, United States; Nasser M. Nasrabadi, U.S. Army Research Laboratory, United States; Thomas S. Huang, University of Illinois Urbana-Champaign, United States; Yanning Zhang, Northwestern Polytechnical University, China

MLSP-P5.9: PAC-BAYESIAN APPROACH FOR MINIMIZATION OF PHONEME ERROR RATE 2224 Joseph Keshet, David McAllester, Tamir Hazan, Toyota Technological Institute at Chicago, United States

MLSP-P5.10: CLOSED-FORM EXPRESSIONS VS. BIC: A COMPARISON FOR SPEAKER 2228 CLUSTERING

Themos Stafylakis, Institute for language and Speech Processing, Greece; Xavier Anguera, Telefonica Research, Spain; Vassilis Katsouros, George Carayannis, Institute for Language and Speech Processing, Greece

MLSP-P5.11: AUTOMATIC AUDIO TAG CLASSIFICATION VIA SEMI-SUPERVISED 2232 CANONICAL DENSITY ESTIMATION

Jun Takagi, Tokyo Institute of Technology, Japan; Yasunori Ohishi, Akisato Kimura, NTT Communication Science Laboratories, Japan; Masashi Sugiyama, Makoto Yamada, Tokyo Institute of Technology, Japan; Hirokazu Kameoka, NTT Communication Science Laboratories, Japan 


\section{MLSP-P6: SIGNAL DETECTION AND CLASSIFICATION}

MLSP-P6.1: NONPARAMETRIC BAYESIAN FEATURE SELECTION FOR MULTI-TASK

LEARNING

Hui Li, Signal Innovations Group Inc., United States; Xuejun Liao, Lawrence Carin, Duke University, United States

MLSP-P6.2: DISCRIMINATIVE SIMPLIFICATION OF MIXTURE MODELS

2240

Yossi Bar-Yosef, Yuval Bistritz, Tel Aviv University, Israel

MLSP-P6.3: OUTLIER-AWARE ROBUST CLUSTERING

Pedro Forero, Vassilis Kekatos, Georgios B. Giannakis, University of Minnesota, United States

MLSP-P6.4: ONLINE LEARNING WITH MINORITY CLASS RESAMPLING

Michael Pekala, Ashley Llorens, The Johns Hopkins University Applied Physics Laboratory, United States

MLSP-P6.5: LEARNING A DISCRIMINATIVE VISUAL CODEBOOK USING HOMONYM 2252

SCHEME

Seungryul Baek, Chang.D. Yoo, Sungrack Yun, Korea Advanced Institute of Science and Technology, Republic of Korea

MLSP-P6.6: MODIFIED EMBEDDING FOR MULTI-REGIME DETECTION IN 2256 NONSTATIONARY STREAMING DATA

Evan Kriminger, Jose C. Principe, University of Florida, United States; Choudur Lakshminarayan, Hewlett-Packard Laboratories, United States

MLSP-P6.7: HOW EFFICIENT IS ESTIMATION WITH MISSING DATA? 2260 Seliz G. Karadogan, Technical University of Denmark, Denmark; Letizia Marchegiani, Sapienza / University of Rome, Italy; Lars Kai Hansen, Jan Larsen, Technical University of Denmark, Denmark

MLSP-P6.8: COMBINING GENERIC AND CLASS-SPECIFIC CODEBOOKS FOR OBJECT 2264 CATEGORIZATION AND DETECTION

Hong Pan, Southeast University, China; YaPing Zhu, University of California San Diego, China; LiangZheng Xia, Southeast University, China; Truong Q. Nguyen, University of California San Diego, United States

MLSP-P6.9: DETECTION OF ANOMALOUS EVENTS FROM UNLABELED SENSOR DATA 2268 IN SMART BUILDING ENVIRONMENTS

Padmini Jaikumar, Purdue University, United States; Aca Gacic, Robert Bosch LLC, United States; Burton Andrews, JPMorgan Chase \& Co, United States; Michael Dambier, Robert Bosch GmbH, Germany

MLSP-P6.10: PROBABILISTIC DISTANCE SVM WITH HELLINGER-EXPONENTIAL 2272 KERNEL FOR SOUND EVENT CLASSIFICATION

Huy Dat Tran, Haizhou Li, Institute for Infocomm Research / Agency of Science Technology And Research, Singapore

MLSP-P6.11: SAMPLING ON LOCALLY DEFINED PRINCIPAL MANIFOLDS 2276

Erhan Bas, Deniz Erdogmus, Northeastern University, United States

\section{MMSP-L1: JOINT AUDIO VISUAL PROCESSING}

MMSP-L1.1: AUDIO-VISUAL SYNCHRONIZATION RECOVERY IN MULTIMEDIA CONTENT

Jong-Seok Lee, Touradj Ebrahimi, Swiss Federal Institute of Technology in Lausanne, Switzerland

MMSP-L1.2: UNSUPERVISED EXTRACTION OF AUDIO-VISUAL OBJECTS

Anna Llagostera Casanovas, Pierre Vandergheynst, Ecole Polytechnique Fédérale de Lausanne, Switzerland

MMSP-L1.3: TRACKING CHANGES IN CONTINUOUS EMOTION STATES USING BODY 2288 LANGUAGE AND PROSODIC CUES

Angeliki Metallinou, Athanasios Katsamanis, University of Southern California, United States; Yun Wang, Carnegie Mellon University, United States; Shrikanth S. Narayanan, University of Southern California, United States 


\section{HEARING}

Pubudu Madhawa Silva, Thrasyvoulos N. Pappas, Northwestern University, United States; Joshua Atkins, James E. West, The

Johns Hopkins University, United States

MMSP-L1.5: VOXEL-BASED VITERBI ACTIVE SPEAKER TRACKING (V-VAST) WITH 2296 BEST VIEW SELECTION FOR VIDEO LECTURE POST-PRODUCTION

Damien Kelly, Anil Kokaram, Frank Boland, Trinity College Dublin, Ireland

MMSP-L1.6: BAYESIAN INTEGRATION OF AUDIO AND VISUAL INFORMATION FOR 2300 MULTI-TARGET TRACKING USING A CB-MEMBER FILTER

Reza Hoseinnezhad, RMIT University, Australia; Ba-Ngu Vo, Ba-Tuong Vo, The University of Western Australia, Australia;

David Suter, The University of Adelaide, Australia

\section{MMSP-L2: MULTIMEDIA INDEXING AND RETRIEVAL}

MMSP-L2.1: EFFICIENT SEARCH OF MUSIC PITCH CONTOURS USING WAVELET TRANSFORMS AND SEGMENTED DYNAMIC TIME WARPING

Woojay Jeon, Changxue Ma, Motorola, United States

MMSP-L2.2: COST-SENSITIVE STACKING FOR AUDIO TAG ANNOTATION AND 2308 RETRIEVAL

Hung-Yi Lo, Ju-Chiang Wang, Hsin-Min Wang, Institute of Information Science / Academia Sinica, Taiwan; Shou-De Lin, National Taiwan University, Taiwan

MMSP-L2.4: A LOW BIT RATE VOCABULARY CODING SCHEME FOR MOBILE 2316 LANDMARK SEARCH Rongrong Ji, Harbin Institute of Technology, China; Ling-Yu Duan, Jie Chen, Peking University, China; Hongxun Yao, Harbin Institute of Technology, China; Wen Gao, Peking University, China

MMSP-L2.5: RAPID IMAGE RETRIEVAL FOR MOBILE LOCATION RECOGNITION 2320

Georg Schroth, Anas Al-Nuaimi, Robert Huitl, Florian Schweiger, Eckehard Steinbach, Technische Universität München, Germany

MMSP-L2.6: TEMPORAL RECURRENCE HASHING ALGORITHM FOR MINING 2324 COMMERCIALS FROM MULTIMEDIA STREAMS

Xiaomeng Wu, Shin'ichi Satoh, National Institute of Informatics, Japan

\section{MMSP-P1: MULTIMEDIA COMMUNICATIONS AND NETWORKING}

MMSP-P1.1: PERFORMANCE EVALUATION OF RAPTOR AND RANDOM LINEAR CODES 2328 FOR H.264/AVC VIDEO TRANSMISSION OVER DVB-H NETWORKS

Sajid Nazir, Dejan Vukobratovic, Vladimir Stankovic, University of Strathclyde, United Kingdom

MMSP-P1.2: APPLICATION CONTROL FOR FAST ADAPTIVE ERROR RESILIENT H.264/AVC 2332 STREAMING OVER IP WIRELESS NETWORKS

Catherine Lamy-Bergot, Benjamin Gadat, Thales Communications S.A., France

MMSP-P1.3: A CROSS-LAYER OPTIMIZATION FOR ENERGY-EFFICIENT MAC 2336 PROTOCOL WITH DELAY AND RATE CONSTRAINTS

Haksub Kim, Hyungkeuk Lee, Sanghoon Lee, Yonsei University, Republic of Korea 
MMSP-P1.4: OPTIMAL POWER ALLOCATION AND JOINT SOURCE-CHANNEL CODING

FOR WIRELESS DS-CDMA VISUAL SENSOR NETWORKS USING THE NASH BARGAINING

SOLUTION

Katerina Pandremmenou, Lisimachos P. Kondi, Konstantinos E. Parsopoulos, University of Ioannina, Greece

MMSP-P1.5: INCENTIVE MECHANISM IN WIRELESS MULTICAST

Bo Hu, H. Vicky Zhao, Hai Jiang, University of Alberta, Canada

MMSP-P1.6: PRICING GAME AND EVOLUTION DYNAMICS FOR MOBILE VIDEO 2348 STREAMING

Wan-Yi Lin, K. J. Ray Liu, University of Maryland College Park, United States

MMSP-P1.7: ADAPTIVE SCALABLE LAYER FILTERING PROCESS FOR VIDEO 2352 SCHEDULING OVER WIRELESS NETWORKS BASED ON MAC BUFFER MANAGEMENT

Nesrine Changuel, Alcatel Lucent bell Labs, France; Nicholas Mastronarde, Mihaela van der Schaar, University of California Los Angeles, United States; Bessem Sayadi, Alcatel Lucent bell Labs, France; Michel Kieffer, LTCI, CNRS-Télécom Paris tec, France

MMSP-P1.8: CONTENT-AWARE TCP-FRIENDLY CONGESTION CONTROL FOR 2356 MULTIMEDIA TRANSMISSION

Hsien-Po Shiang, Mihaela van der Schaar, University of California Los Angeles, United States

MMSP-P1.9: MULTI-GRAPH REGULARIZATION FOR EFFICIENT DELIVERY OF USER 2360 GENERATED CONTENT IN ONLINE SOCIAL NETWORKS

Jacob Chakareski, Ecole Polytechnique Fédérale de Lausanne, Switzerland

MMSP-P1.10: AUDIO WATERMARKING FOR ACOUSTIC PROPAGATION IN REVERBERANT 2364 ENVIRONMENTS

Giovanni Del Galdo, Juliane Borsum, Tobias Bliem, Alexandra Craciun, Stefan Krägeloh, Fraunhofer Institute for Integrated Circuits (IIS), Germany

\section{MMSP-P2: MULTIMEDIA ANALYSIS, CLASSIFICATION, AND RECOGNITION}

MMSP-P2.1: ESTIMATION OF ORDINAL APPROACH-AVOIDANCE LABELS IN DYADIC 2368 INTERACTIONS: ORDINAL LOGISTIC REGRESSION APPROACH

Viktor Rozgic, Bo Xiao, Athanasios Katsamanis, Brian Baucom, Panayiotis Georgiou, Shrikanth S. Narayanan, University of Southern California, United States

MMSP-P2.2: A HIERARCHICAL STATIC-DYNAMIC FRAMEWORK FOR EMOTION 2372 CLASSIFICATION

Emily Mower, Shrikanth S. Narayanan, University of Southern California, United States

MMSP-P2.3: A SUPERVISED APPROACH TO MOVIE EMOTION TRACKING

Nikos Malandrakis, Alexandros Potamianos, Technical University of Crete, Greece; Georgios Evangelopoulos, Athanasia

Zlatintsi, National Technical University of Athens, Greece

MMSP-P2.4: AUTOMATIC VIDEO ANNOTATION VIA HIERARCHICAL TOPIC TRAJECTORY 2380 MODEL CONSIDERING CROSS-MODAL CORRELATIONS

Takuho Nakano, The University of Tokyo, Japan; Akisato Kimura, Hirokazu Kameoka, NTT Communication Science Laboratories, Japan; Shigeki Miyabe, Shigeki Sagayama, Nobutaka Ono, The University of Tokyo, Japan; Kunio Kashino, NTT Communication Science Laboratories, Japan; Takuya Nishimoto, The University of Tokyo, Japan

MMSP-P2.5: KERNEL CROSS-MODAL FACTOR ANALYSIS FOR MULTIMODAL 2384 INFORMATION FUSION

Yongjin Wang, Ling Guan, Anastasios Venetsanopoulos, Ryerson University, Canada

MMSP-P2.6: BELIEF THEORETIC METHODS FOR SOFT AND HARD DATA FUSION 
Panikos Heracleous, Norihiro Hagita, ATR, Intelligent Robotics and Communication Laboratories, Japan

MMSP-P2.8: CONTINUOUS AUDIO ANALYTICS BY HMM AND VITERBI DECODING 2396

$V$ Ramasubramanian, R Karthik, S Thiyagarajan, Siemens Corporate Research \& Technologies India, India; Srikanth Cherla,

Universitat Pompeu Fabra, Spain

MMSP-P2.9: WHEN CODEWORD FREQUENCY MEETS GEOGRAPHICAL LOCATION.

2400

Rongrong Ji, Harbin Institute of Technology, China; Ling-Yu Duan, Jie Chen, Peking University, China; Hongxun Yao, Harbin Institute of Technology, China; Wen Gao, Peking University, China

MMSP-P2.10: USER VERIFICATION: MATCHING THE UPLOADERS OF VIDEOS ACROSS 2404 ACCOUNTS

Howard Lei, Jaeyoung Choi, Adam Janin, Gerald Friedland, International Computer Science Institute, United States

\section{MMSP-P3: MULTIMEDIA PERCEPTION, QUALITY, EVALUATION, AND DATA HIDING}

MMSP-P3.1: PINNA SENSITIVITY PATTERNS REVEAL REFLECTING AND DIFFRACTING 2408 SURFACES THAT GENERATE THE FIRST SPECTRAL NOTCH IN THE FRONT MEDIAN PLANE

Parham Mokhtari, Hironori Takemoto, Ryouichi Nishimura, Hiroaki Kato, National Institute of Information and Communications Technology, Japan

MMSP-P3.2: SPATIALLY SPARSED COMMON SPATIAL PATTERN TO IMPROVE BCI 2412 PERFORMANCE

Mahnaz Arvaneh, Nanyang Technological University, Singapore; Cuntai Guan, Kai Keng Ang, Institute for Infocomm Research, Singapore; Hiok Chai Quek, Nanyang Technological University, Singapore

MMSP-P3.3: CROWDMOS: AN APPROACH FOR CROWDSOURCING MEAN OPINION 2416 SCORE STUDIES

Flavio Ribeiro, University of Sao Paulo, Brazil; Dinei Florencio, Cha Zhang, Michael Seltzer, Microsoft Research, United States

MMSP-P3.4: EVALUATION OF OBJECTIVE MEASURES FOR QUALITY ASSESSMENT OF 2420 REVERBERANT SPEECH

Kostas Kokkinakis, Philipos Loizou, The University of Texas at Dallas, United States

MMSP-P3.5: THE PROPOSAL OF QUANTIFICATION METHOD OF SPEAKER 2424 IDENTIFICATION ACCURACY FOR SPEECH COMMUNICATION SERVICE

Noritsugu Egi, Takanori Hayashi, Akira Takahashi, Nippon Telegraph and Telephone Corporation, Japan

MMSP-P3.6: AN AUTOMATED SINGING EVALUATION METHOD FOR KARAOKE SYSTEMS 2428

Wei-Ho Tsai, Hsin-Chieh Lee, National Taipei University of Technology, Taiwan

MMSP-P3.7: LOSSLESS AUDIO HIDING METHOD FOR SYNCHRONOUS AUDIO-VIDEO 2432 CODING

Weiwei Chen, Jin Li, Moncef Gabbouj, Jarmo Takala, Tampere University of Technology, Finland

MMSP-P3.8: PERCEPTUAL VIDEO ENCRYPTION USING MULTIPLE 8×8 TRANSFORMS

IN H.264 AND MPEG-4

Siu-Kei Au Yeung, Shuyuan Zhu, Bing Zeng, The Hong Kong University of Science and Technology, Hong Kong SAR of China

MMSP-P3.9: A NEW DATA HIDING METHOD USING ANGLE QUANTIZATION INDEX 2440 MODULATION IN GRADIENT DOMAIN

Ehsan Nezhadarya, Jane Wang, Rabab K. Ward, The University of British Columbia, Canada

MMSP-P3.10: IMPROVED DCT COEFFICIENT ANALYSIS FOR FORGERY LOCALIZATION 2444 IN JPEG IMAGES

Tiziano Bianchi, Alessia De Rosa, Alessandro Piva, University of Firenze, Italy 


\section{SAM-L1: DETECTION AND ESTIMATION}

SAM-L1.1: PERIODIC CRB FOR NON-BAYESIAN PARAMETER ESTIMATION

Tirza Routtenberg, Joseph Tabrikian, Ben-Gurion University of the Negev, Israel

SAM-L1.2: MAXIMUM A POSTERIORI BASED REGULARIZATION PARAMETER SELECTION 2452

Ashkan Panahi, Mats Viberg, Chalmers University of Technology, Sweden

SAM-L1.3: CORRELOGRAM TEMPLATE MATCHING FOR TIME-DELAY ESTIMATION

Bowon Lee, Ton Kalker, Ronald W. Schafer, Hewlett-Packard Laboratories, United States

SAM-L1.4: APPLICATIONS OF SHORT SPACE-TIME FOURIER ANALYSIS IN DIGITAL 2460 ACOUSTICS

Francisco Pinto, Martin Vetterli, Ecole Polytechnique Fédérale de Lausanne, Switzerland

SAM-L1.5: ANALYTICAL PERFORMANCE ASSESSMENT OF 1-D STRUCTURED LEAST 2464 SQUARES

Florian Roemer, Martin Haardt, Ilmenau University of Technology, Germany

\section{SAM-L2: SOURCE LOCALIZATION}

SAM-L2.1: TOA LOCALIZATION IN THE PRESENCE OF RANDOM SENSOR POSITION 2468 ERRORS

Zhenhua Ma, K.C. Ho, University of Missouri, United States

SAM-L2.2: TARGET LOCALIZATION WITH NLOS CIRCULARLY REFLECTED AOAS

2472

Xiufeng Song, Peter Willett, Shengli Zhou, University of Connecticut, United States

SAM-L2.3: MULTIPLE SPEAKER TRACKING USING A MICROPHONE ARRAY BY

\section{COMBINING AUDITORY PROCESSING AND A GAUSSIAN MIXTURE CARDINALIZED} PROBABILITY HYPOTHESIS DENSITY FILTER

Axel Plinge, Leibniz Research Centre for Working Environment and Human Factors, Germany; Daniel Hauschildt, Marius H. Hennecke, Gernot A. Fink, TU Dortmund University, Germany

SAM-L2.4: RSS-BASED SENSOR LOCALIZATION WITH UNKNOWN TRANSMIT POWER

Reza M. Vaghefi, Mohammad Reza Gholami, Erik G. Ström, Chalmers University of Technology, Sweden

SAM-L2.5: CRAMER-RAO BOUNDS FOR POWER DELAY PROFILE FINGERPRINTING

BASED POSITIONING

Turgut Öktem, Dirk Slock, EURECOM, France

SAM-L2.6: PRACTICAL LIMITS IN RSS-BASED POSITIONING

Richard Martin, Amanda Sue King, Ryan Thomas, The Air Force Institute of Technology, United States; Jason Pennington,

Miami University, United States

\section{SAM-L3: SENSOR NETWORKS}

SAM-L3.1: OPTIMAL POWER ALLOCATION IN DISTRIBUTED MULTIPLE-RADAR 2492 CONFIGURATIONS

Hana Godrich, Princeton University / Rutgers University, United States; Athina Petropulu, Rutgers University, United States; H. Vincent Poor, Princeton University, United States 
SAM-L3.4: A SIMPLE NETWORK-POWER-SAVING RESOURCE ALLOCATION METHOD 2504 FOR OFDMA CELLULAR NETWORKS WITH MULTIPLE RELAYS

Jingon Joung, Sumei Sun, Institute for Infocomm Research, Singapore

SAM-L3.5: ORDERING FOR ENERGY EFFICIENT ESTIMATION AND OPTIMIZATION IN 2508 SENSOR NETWORKS

Rick Blum, Lehigh University, United States

SAM-L3.6: A KNAPSACK PROBLEM FORMULATION FOR RELAY SELECTION IN SECURE 2512 COOPERATIVE WIRELESS COMMUNICATION

Shuangyu Luo, Rutgers University, United States; Hana Godrich, Princeton University / Rutgers University, United States; Athina Petropulu, Rutgers University, United States; H. Vincent Poor, Princeton University, United States

\section{SAM-L4: SIGNAL SEPARATION}

SAM-L4.1: SEPARATION AND TRACKING OF MULTIPLE SPEAKERS IN A REVERBERANT 2516 ENVIRONMENT USING A MULTIPLE MODEL PARTICLE FILTER GLIMPSING METHOD

Alireza Masnadi-Shirazi, Bhaskar D. Rao, University of California San Diego, United States

SAM-L4.2: JOINT BLIND SOURCE SEPARATION FROM SECOND-ORDER STATISTICS: 2520 NECESSARY AND SUFFICIENT IDENTIFIABILITY CONDITIONS

Javier Via, University of Cantabria, Spain; Matthew Anderson, Xi-Lin Li, Tülay Adali, University of Maryland Baltimore County, United States

SAM-L4.3: REGULARIZED GRADIENT ALGORITHM FOR NON-NEGATIVE INDEPENDENT 2524 COMPONENT ANALYSIS

Wendyam Serge Boris Ouedraogo, Commissariat à l'Energie Atomique et aux Energies Alternatives, France; Meriem Jaidane, Ecole Nationale d'Ingénieurs de Tunis, Tunisia; Antoine Souloumiac, Commissariat à l'Energie Atomique et aux Energies Alternatives, France; Christian Jutten, Université Joseph Fourier / Grenoble et Institut Universitaire de France, France

SAM-L4.4: A FLEXIBLE SPEECH DISTORTION WEIGHTED MULTI-CHANNEL WIENER 2528 FILTER FOR NOISE REDUCTION IN HEARING AIDS

Kim Ngo, Marc Moonen, Katholieke Universiteit Leuven, Belgium; Sфren Holdt Jensen, Aalborg University, Denmark; Jan Wouters, Katholieke Universiteit Leuven, Belgium

SAM-L4.5: HYBRID PROBABILISTIC ADAPTATION MODE CONTROLLER FOR 2532 GENERALIZED SIDELOBE CANCELLER-BASED TARGET-DIRECTIONAL SPEECH ENHANCEMENT

Seon Man Kim, Hong Kook Kim, Gwangju Institute of Science and Technology, Republic of Korea

SAM-L4.6: EXPLOITING MULTIPATH FOR BLIND SOURCE SEPARATION WITH SENSOR 2536 ARRAYS

Giuseppe Fabrizio, Defence Science and Technology Organisation, Australia; Alfonso Farina, Selex Sistemi Integrati, Italy

\section{SAM-P1: DIRECTION-OF-ARRIVAL ESTIMATION}

SAM-P1.1: PRIOR KNOWLEDGE-BASED DIRECTION OF ARRIVAL ESTIMATION. 2540

Petter Wirfält, Magnus Jansson, KTH - Royal Institute of Technology, Sweden; Guillaume Bouleux, Saint Etienne University,

France; Petre Stoica, Uppsala University, Sweden

SAM-P1.2: OPTIMIZATION OF THE ANTENNA ARRAY GEOMETRY BASED ON A BAYESIAN 2544 DOA ESTIMATION CRITERION

Houcem Gazzah, University of Sharjah, United Arab Emirates; Jean-Pierre Delmas, Telecom SudParis, France 
PARTLY-CALIBRATED ARRAYS

Pouyan Parvazi, Marius Pesavento, Alex B. Gershman, TU Darmstadt, Germany

SAM-P1.5: CONVEX RELAXATION APPROACHES TO MAXIMUM LIKELIHOOD DOA 2556 ESTIMATION IN ULA'S AND UCA'S WITH UNKNOWN MUTUAL COUPLING

Kehu Yang, Shu Cai, Xidian University, China; Zhi-Quan Luo, University of Minnesota, United States

SAM-P1.6: EXTENDED ARRAY METHOD FOR DETECTION AND DIRECTION FINDING 2560

OF A WEAK LINEAR FREQUENCY MODULATED SIGNAL

Songsri Sirianunpiboon, Defence Science and Technology Organisation, Australia

SAM-P1.7: NEAR-FIELD ARRAY SHAPE CALIBRATION

Shuang Wan, Pei-Jung Chung, Bernard Mulgrew, University of Edinburgh, United Kingdom

SAM-P1.8: GAIN AND PHASE AUTOCALIBRATION FOR UNIFORM RECTANGULAR ARRAYS 2568

Philipp Heidenreich, Abdelhak M. Zoubir, Technische Universität Darmstadt, Germany

SAM-P1.9: A PASSIVE COUPLING MATRIX DESIGN FOR IMPROVED RESOLUTION

SMALL APERTURE DIRECTION FINDING

Guohua Wang, Temasek Laboratories NTU, Singapore; Joni Polili Lie, Nanyang Technological University, Singapore; Chong-

Meng Samson See, DSO National Laboratories, Singapore

SAM-P1.10: OPERATIONAL PERFORMANCES OF A MUSIC ALGORITHM ROBUST TO 2576 OUTLIERS

Anne Ferréol, Thales Communications, France; Pascal Larzabal, SATIE / ENS Cachan / CNRS Universud, France

SAM-P1.11: SUBSPACE-BASED DOA ESTIMATION USING FRACTIONAL LOWER ORDER 2580 STATISTICS

K. V. S. Hari, V Lalitha, Indian Institute of Science, India

\section{SAM-P2: SOURCE LOCALIZATION}

SAM-P2.1: EFFICIENT SEMIDEFINITE RELAXATION FOR ROBUST GEOLOCATION OF 2584 UNKNOWN EMITTER BY A SATELLITE CLUSTER USING TDOA AND FDOA MEASUREMENTS

Kehu Yang, Lizhong Jiang, Xidian University, China; Zhi-Quan Luo, University of Minnesota, United States

SAM-P2.2: A QUADRATIC CONSTRAINT SOLUTION METHOD FOR TDOA AND FDOA 2588 LOCALIZATION

Fucheng Guo, National University of Defense Technology, China; K.C. Ho, University of Missouri, United States

SAM-P2.3: REAL TIME SPEAKER LOCALIZATION AND DETECTION SYSTEM FOR 2592 CAMERA STEERING IN MULTIPARTICIPANT VIDEOCONFERENCING ENVIRONMENTS

Amparo Marti, Maximo Cobos, Jose J. Lopez, Universidad Politécnica de Valencia, Spain

SAM-P2.4: A SPACE TIME ARRAY PROCESSING FOR PASSIVE GEOLOCALIZATION OF 2596 RADIO TRANSMITTERS

Jonathan Bosse, Anne Ferréol, Thales Communications / SATIE laboratory, France; Pascal Larzabal, SATIE Laboratory, France

SAM-P2.5: A NEAR-OPTIMAL LEAST SQUARES SOLUTION TO RECEIVED SIGNAL 2600 STRENGTH DIFFERENCE BASED GEOLOCATION

Sichun Wang, Robert Inkol, Defence R\&D Canada, Canada

SAM-P2.6: GAUSSIAN MIXTURE MODELING FOR SOURCE LOCALIZATION 2604

John Flåm, Norwegian University of Science and Technology, Norway; Joakim Jaldén, Saikat Chatterjee, KTH - Royal Institute of Technology, Sweden 
Joni Polili Lie, Nanyang Technological University, Singapore; Thierry Blu, The Chinese University of Hong Kong, Hong Kong SAR of China; Chong-Meng Samson See, DSO National Laboratories, Singapore

SAM-P2.8: COOPERATIVE MOBILE NETWORK LOCALIZATION VIA SUBSPACE 2612 TRACKING

Hadi Jamali Rad, Alon Amar, Delft University of Technology, Netherlands; Geert Leus, TU Delft, Netherlands

SAM-P2.9: MULTI-SOURCE TDOA ESTIMATION USING SNR-BASED ANGULAR SPECTRA 2616

Charles Blandin, Emmanuel Vincent, Alexey Ozerov, INRIA, France

SAM-P2.10: FROM DIRECTION OF ARRIVAL ESTIMATES TO LOCALIZATION OF PLANAR 2620 REFLECTORS IN A TWO DIMENSIONAL GEOMETRY

Antonio Canclini, Politecnico di Milano, Italy; Paolo Annibale, University Erlangen-Nuremberg, Germany; Fabio Antonacci, Augusto Sarti, Politecnico di Milano, Italy; Rudolf Rabenstein, University Erlangen-Nuremberg, Germany; Stefano Tubaro,

Politecnico di Milano, Italy

SAM-P2.11: A MODEL-BASED AUDITORY SCENE ANALYSIS APPROACH AND ITS 2624 APPLICATION TO SPEECH SOURCE LOCALIZATION

Vaclav Bouse, Siemens Audiologische Technik, Germany; Rainer Martin, Ruhr-Universität Bochum, Germany

\section{SAM-P3: BEAMFORMING}

SAM-P3.1: WORST-CASE BASED ROBUST ADAPTIVE BEAMFORMING FOR 2628 GENERAL-RANK SIGNAL MODELS USING POSITIVE SEMI-DEFINITE COVARIANCE CONSTRAINT

Haihua Chen, Nankai University, China; Alex B. Gershman, Darmstadt University of Technology, Germany

SAM-P3.2: INTEGRATED SIDELOBE LEVEL OF SETS OF ROTATED LEGENDRE 2632 SEQUENCES

Javier Haboba, Riccardo Rovatti, University of Bologna, Italy; Gianluca Setti, University of Ferrara, Italy

SAM-P3.3: NON-DATA-AIDED ADAPTIVE BEAMFORMING ALGORITHM BASED ON THE 2636 WIDELY LINEAR AUXILIARY VECTOR FILTER

Nuan Song, Jens Steinwandt, Ilmenau University of Technology, Germany; Lei Wang, Rodrigo C. de Lamare, University of York, United Kingdom; Martin Haardt, Ilmenau University of Technology, Germany

SAM-P3.4: ROBUST ADAPTIVE BEAMFORMING BASED ON JOINTLY ESTIMATING 2640 COVARIANCE MATRIX AND STEERING VECTOR

Yujie Gu, Amir Leshem, Bar-Ilan University, Israel

SAM-P3.5: A CLT ON THE SINR OF THE DIAGONALLY LOADED CAPON/MVDR 2644 BEAMFORMER

Francisco Rubio, Xavier Mestre, Centre Tecnològic de Telecomunicacions de Catalunya (CTTC), Spain; Walid Hachem, Télécom ParisTech / CNRS, France

SAM-P3.6: THIRD ORDER WIDELY NON LINEAR VOLTERRA MVDR BEAMFORMING 2648

Pascal Chevalier, CNAM, France; Abdelkader Oukaci, Jean-Pierre Delmas, Telecom SudParis, France

SAM-P3.7: ACOUSTIC VECTOR-SENSOR BEAMFORMING IN THE PRESENCE OF FLOW 2652 NOISE

Nan Zou, DSO National Laboratories, Singapore; Arye Nehorai, Washington University in St. Louis, United States; Ing Nam Goh, DSO National Laboratories, Singapore 


\section{TO POWER CONSTRAINTS}

Marius Caus, Ana Isabel Perez-Neira, Universitat Politècnica de Catalunya, Spain

SAM-P3.10: ANALOG ANTENNA COMBINING IN MULTIUSER OFDM SYSTEMS:

BEAMFORMING DESIGN AND POWER ALLOCATION

Alfredo Nazábal, Javier Vía, Ignacio Santamaría, University of Cantabria, Spain

SAM-P3.11: DISTRIBUTED LCMV BEAMFORMING IN WIRELESS SENSOR NETWORKS 2668 WITH NODE-SPECIFIC DESIRED SIGNALS

Alexander Bertrand, Marc Moonen, Katholieke Universiteit Leuven, Belgium

\section{SAM-P4: APPLICATIONS OF SENSOR ARRAY AND MULTICHANNEL PROCESSING}

SAM-P4.1: MULTIPLE-MEASUREMENT VECTOR MODEL AND ITS APPLICATION TO 2672 THROUGH-THE-WALL RADAR IMAGING Jie Yang, Abdesselam Bouzerdoum, Fok Tivive, School of Electrical Computer and Telecommunications Engineering, Australia; Moeness G. Amin, Centre for Advanced Communications, United States

SAM-P4.2: MULTIPATH MODEL AND EXPLOITATION IN THROUGH-THE-WALL RADAR 2676 AND URBAN SENSING

Pawan Setlur, Moeness G. Amin, Fauzia Ahmad, Villanova University, United States

SAM-P4.3: A FAST TRANSFORM FOR ACOUSTIC IMAGING WITH SEPARABLE ARRAYS 2680

Flavio Ribeiro, Vitor Nascimento, University of Sao Paulo, Brazil

SAM-P4.4: ADAPTIVE DETECTION OF MULTIPLE POINT-LIKE TARGETS WITH CONIC 2684 ACCEPTANCE

Chengpeng Hao, Chinese Academy of Sciences, China; Francesco Bandiera, Universita del Salento, Italy; Jun Yang, Chaohuan Hou, Chinese Academy of Sciences, China

SAM-P4.5: COMPUTATIONALLY EFFICIENT REGULARIZED ACOUSTIC IMAGING 2688

Flavio Ribeiro, Vitor Nascimento, University of Sao Paulo, Brazil

SAM-P4.6: MULTI-RANK PROCESSING FOR PASSIVE RANGING IN UNDERWATER 2692 ACOUSTIC ENVIRONMENTS SUBJECT TO SPATIAL COHERENCE LOSS

Hongya Ge, New Jersey Institute of Technology, United States; Ivars Kirsteins, Naval Undersea Warfare Center, United States

SAM-P4.7: FEATURE SELECTION BASED ON MULTIPLE KERNEL LEARNING FOR 2696 SINGLE-CHANNEL SOUND SOURCE LOCALIZATION USING THE ACOUSTIC TRANSFER FUNCTION

Ryoichi Takashima, Tetsuya Takiguchi, Yasuo Ariki, Kobe University, Japan

SAM-P4.8: A NOVEL PROBE PROCESSING METHOD FOR UNDERWATER 2700 COMMUNICATION BY PASSIVE-PHASE CONJUGATION

Guosong Zhang, Norwegian University of Science and Technology, Norway; Jens M. Hovem, SINTEF ICT, Norway; Hefeng Dong, Norwegian University of Science and Technology, Norway; P. A. van Walree, Norwegian Defence Research Establishment, Norway

SAM-P4.9: DATA DRIVEN MODEL BASED LEAST SQUARES IMAGE RECONSTRUCTION 2704 FOR RADIO ASTRONOMY

Stefan Wijnholds, ASTRON, Netherlands; Alle-Jan van der Veen, Delft University of Technology, Netherlands

SAM-P4.10: DETECTION OF AUDITORY STIMULUS ONSET IN THE PONTINE 2708 NUCLEUS USING A MULTICHANNEL MULTI-UNIT ACTIVITY ELECTRODE

Majd Zreik, Ytai Ben-Tsvi, Aryeh Taub, Rakefet Ofek Almog, Hagit Messer, Tel Aviv University, Israel 


\section{SAM-P5: DETECTION AND ESTIMATION}

SAM-P5.1: SOURCE NUMBER ESTIMATION IN IMPULSIVE NOISE ENVIRONMENTS

USING BOOTSTRAP TECHNIQUES AND ROBUST STATISTICS

Zhihua Lu, Yacine Chakhchoukh, Abdelhak M. Zoubir, Technische Universität Darmstadt, Germany

SAM-P5.2: TIME DELAY ESTIMATION IN THE TIME-FREQUENCY DOMAIN BASED ON A 2716 LINE DETECTION APPROACH

Andreas Sandmair, Mario Lietz, Johannes Stefan, Fernando Puente León, Karlsruhe Institute of Technology, Germany

SAM-P5.3: STABLE SUBSPACE TRACKING ALGORITHM BASED ON SIGNED URV DECOMPOSITION

Mu Zhou, Alle-Jan van der Veen, Delft University of Technology, Netherlands

SAM-P5.4: SEQUENTIAL CRAMÉR-RAO LOWER BOUNDS FOR BISTATIC RADAR SYSTEMS

Pietro Stinco, Maria Greco, Fulvio Gini, University of Pisa, Italy; Alfonso Farina, SELEX - Sistemi Integrati, Italy

SAM-P5.5: ARRAY-BASED GNSS ACQUISITION IN THE PRESENCE OF COLORED NOISE 2728

Javier Arribas, Carles Fernández-Prades, Pau Closas, Centre Tecnològic de Telecomunicacions de Catalunya (CTTC), Spain

SAM-P5.6: COMPUTING THE NONNEGATIVE 3-WAY TENSOR FACTORIZATION USING 2732 TIKHONOV REGULARIZATION

Jean-Philip Royer, Pierre Comon, I3S, France; Nadège Thirion-Moreau, ISITV LSEET, France

SAM-P5.7: ROBUST DIRECTION ESTIMATION OF UWB SOURCES IN LAGUERRE-GAUSS

2736 BEAMSPACES

Elio D. Di Claudio, Giovanni Jacovitti, Alberto Laurenti, University of Rome La Sapienza, Italy

SAM-P5.8: AN INTRODUCTION TO CONSISTENT GRAPHS AND THEIR SIGNAL

2740 PROCESSING APPLICATIONS

Bin Yang, Martin Kreißig, University of Stuttgart, Germany

SAM-P5.9: MUSIC ALGORITHM TO LOCALIZE SOURCES WITH UNKNOWN

DIRECTIVITY IN ACOUSTIC IMAGING

Forooz Shahbazi Avarvand, Andreas Ziehe, Guido Nolte, Fraunhofer Institute FIRST, Germany

SAM-P5.10: SEISMIC WAVES ESTIMATION AND WAVE FIELD DECOMPOSITION WITH 2748 FACTOR GRAPHS

Stefano Maranò, Christoph Reller, Donat Faeh, Hans-Andrea Loeliger, ETH Zürich, Switzerland

SAM-P5.11: TIME PREDICTION OF NON FLAT FADING CHANNELS

2752

Nico Palleit, Tobias Weber, University of Rostock, Germany

\section{SAM-P6: MIMO RADAR AND SPACE-TIME ADAPTIVE PROCESSING}

SAM-P6.1: STATISTICAL RESOLUTION LIMIT FOR SOURCE LOCALIZATION IN A MIMO 2756 CONTEXT

Mohammed Nabil El Korso, Rémy Boyer, Alexandre Renaux, University of Paris-Sud, France; Sylvie Marcos, CNRS, France

SAM-P6.2: MIMO RADAR FOR DIRECTION FINDING WITH EXPLOITATION OF 2760 TIME-FREQUENCY REPRESENTATIONS

Yimin Zhang, Moeness G. Amin, Villanova University, United States

SAM-P6.3: MIMO RADAR DIVERSITY WITH NEYMAN-PEARSON SIGNAL DETECTION IN 2764 NON-GAUSSIAN CIRCUMSTANCE WITH NON-ORTHOGONAL WAVEFORMS

Qian He, Rick Blum, Lehigh University, United States 
SAM-P6.5: SPACE-TIME ADAPTIVE PROCESSING FOR RANGE-FOLDED

SPREAD-DOPPLER RADAR CLUTTER MITIGATION

William Lee, Jeffrey Krolik, Duke University, United States

SAM-P6.6: STATISTICAL ANALYSIS OF MULTI-CHANNEL DETECTION USING DATA FROM 2776 AIRBORNE AESA RADAR

Johan Degerman, Thomas Pernstål, Magnus Gisselfält, Roland Jonsson, Saab AB, Sweden

SAM-P6.7: MIMO RADAR IN THE PRESENCE OF MODELING ERRORS: A CRAMÉR-RAO

2780 BOUND INVESTIGATION

Nguyen Duy Tran, ENS Cachan, France; Alexandre Renaux, Rémy Boyer, University of Paris-Sud, France; Sylvie Marcos, CNRS, France; Pascal Larzabal, ENS Cachan / University of Paris-Sud, France

SAM-P6.8: TRANSMIT BEAMSPACE DESIGN FOR DIRECTION FINDING IN COLOCATED 2784 MIMO RADAR WITH ARBITRARY RECEIVE ARRAY

Arash Khabbazibasmenj, Aboulnasr Hassanien, Sergiy Vorobyov, University of Alberta, Canada

SAM-P6.9: SUBSPACE-BASED DIRECTION FINDING USING TRANSMIT ENERGY 2788 FOCUSING IN MIMO RADAR WITH COLOCATED ANTENNAS

Aboulnasr Hassanien, Sergiy Vorobyov, University of Alberta, Canada

SAM-P6.10: WIDELY DISTRIBUTED MIMO RADAR BEAMFORMING FOR DETECTING 2792 TARGETS WITH SLOW RCS FLUCTUATIONS

Tuomas Aittomaki, Visa Koivunen, Aalto University, Finland

SAM-P6.11: A COMBINATORIAL OPTIMIZATION FRAMEWORK FOR SUBSET SELECTION 2796 IN DISTRIBUTED MULTIPLE-RADAR ARCHITECTURES

Hana Godrich, Princeton University / Rutgers University, United States; Athina Petropulu, Rutgers University, United States; H. Vincent Poor, Princeton University, United States

\section{SAM/SPCOM-P7: RELAY NETWORKS}

SAM/SPCOM-P7.1: DISTRIBUTED BEAMFORMING FOR MULTIUSER PEER-TO-PEER 2800 AND MULTI-GROUP MULTICASTING RELAY NETWORKS

Nils Bornhorst, Marius Pesavento, Alex B. Gershman, Technische Universität Darmstadt, Germany

SAM/SPCOM-P7.2: CAPACITY MAXIMIZATION FOR DISTRIBUTED BEAMFORMING IN 2804 ONE- AND BI-DIRECTIONAL RELAY NETWORKS

Adrian Schad, Alex B. Gershman, TU Darmstadt, Germany; Shahram Shahbazpanahi, University of Ontario Institute of Technology, Canada

SAM/SPCOM-P7.3: SUM-RATE MAXIMIZATION OF TWO-WAY AMPLIFY-AND-FORWARD 2808 RELAY NETWORKS WITH IMPERFECT CHANNEL STATE INFORMATION

Yupeng Jia, Azadeh Vosoughi, University of Rochester, United States

SAM/SPCOM-P7.4: ML DECODING IN DECODE-AND-FORWARD BASED COOPERATIVE 2812 COMMUNICATION SYSTEM

Manav Bhatnagar, Indian Institute of Technology Delhi, India; Are Hjørungnes, University of Oslo, Norway

SAM/SPCOM-P7.5: ROBUST SECONDARY MULTICAST TRANSMIT BEAMFORMING FOR 2816 COGNITIVE RADIO NETWORKS UNDER IMPERFECT CHANNEL STATE INFORMATION Yongwei Huang, Qiang Li, Wing-Kin Ma, Shuzhong Zhang, The Chinese University of Hong Kong, Hong Kong SAR of China 
DUAL-HOP AMPLIFY-AND-FORWARD SYSTEMS

Caijun Zhong, Tharmalingam Ratnarajah, Queen's University Belfast, United Kingdom; Shi Jin, Southeast University, China;

Mathini Sellathurai, Colin Cowan, Queen's University Belfast, United Kingdom

SAM/SPCOM-P7.7: BEAMFORMING DESIGN FOR MULTI-USER TWO-WAY RELAYING 2824

WITH MIMO AMPLIFY AND FORWARD RELAYS

Jianshu Zhang, Florian Roemer, Martin Haardt, Ilmenau University of Technology, Germany

SAM/SPCOM-P7.8: BLIND CHANNEL ESTIMATION FOR MPSK-BASED

2828

AMPLIFY-AND-FORWARD TWO-WAY RELAYING

Saeed Abdallah, Ioannis Psaromiligkos, McGill University, Canada

SAM/SPCOM-P7.9: POWER ALLOCATION FOR ORTHOGONAL AF RELAY SYSTEMS WITH 2832 OUTAGE-BASED QOS CONSTRAINTS

Rooholah Hasanizadeh, Timothy Davidson, McMaster University, Canada

SAM/SPCOM-P7.10: SPACE-TIME BEAMFORMING FOR MULTIUSER WIRELESS RELAY 2836 NETWORKS

Anh Phan, Tuan Hoang, Kha Ha, University of New South Wales, Australia

SAM/SPCOM-P7.11: COORDINATED USER SCHEDULING IN THE MULTI-CELL MIMO 2840 DOWNLINK

Nima Seifi, Michail Matthaiou, Mats Viberg, Chalmers University of Technology, Sweden

\section{SAM-P8: COMPRESSED SENSING AND SPARSE SIGNAL REPRESENTATIONS}

SAM-P8.1: A SPARSE COVARIANCE-BASED METHOD FOR DIRECTION OF ARRIVAL 2844 ESTIMATION

Petre Stoica, Prabhu Babu, Uppsala University, Sweden; Jian Li, University of Florida, United States

SAM-P8.2: DIRECTIONS-OF-ARRIVAL ESTIMATION USING A SPARSE SPATIAL SPECTRUM 2848 MODEL WITH UNCERTAINTY

Jimeng Zheng, Mostafa Kaveh, University of Minnesota Minneapolis, United States

SAM-P8.3: PARAMETER ESTIMATION USING SPARSE RECONSTRUCTION WITH 2852 DYNAMIC DICTIONARIES

Christian Austin, Joshua Ash, Randolph Moses, The Ohio State University, United States

SAM-P8.4: AN APPROACH OF DOA ESTIMATION USING NOISE SUBSPACE WEIGHTED L1 2856 MINIMIZATION

Chundi Zheng, Gang Li, Hao Zhang, Xiqin Wang, Tsinghua University, China

SAM-P8.5: SPARSE FREQUENCY WAVEFORM DESIGN BASED ON PSD FITTING

Guohua Wang, Yilong Lu, Nanyang Technological University, Singapore

SAM-P8.6: SPARSE CHANNEL ESTIMATION WITH LP-NORM AND REWEIGHTED 2864 L1-NORM PENALIZED LEAST MEAN SQUARES

Omid Taheri, Sergiy Vorobyov, University of Alberta, Canada

SAM-P8.7: MULTI IMAGE SUPER RESOLUTION USING COMPRESSED SENSING 2868

Torsten Edeler, Kevin Ohliger, Stephan Hussmann, Alfred Mertins, Westcoast University of Applied Science, Germany

SAM-P8.8: SOURCE LOCALIZATION USING TIME DIFFERENCE OF ARRIVAL WITHIN A 2872 SPARSE REPRESENTATION FRAMEWORK

Ciprian R. Comsa, Alexander M. Haimovich, New Jersey Institute of Technology, United States; Stuart Schwartz, York Dobyns, Princeton University, United States; Jason A. Dabin, U.S. Army Communications-Electronics Research, United States 


\section{ESTIMATION}

Simon Arberet, Ecole Polytechnique Fédérale de Lausanne, Switzerland; Prasad Sudhakar, Rémi Gribonval, INRIA, France

SAM-P8.10: ANALYSIS OF UNKNOWN VELOCITY AND TARGET OFF THE GRID

PROBLEMS IN COMPRESSIVE SENSING BASED SUBSURFACE IMAGING

Mehmet Ali Çagri Tuncer, Ali Cafer Gurbuz, TOBB University of Economics and Technology, Turkey

\section{SPED-P1: SIGNAL PROCESSING EDUCATION}

SPED-P1.1: WINDSK8: A USER INTERFACE FOR THE OMAP-L138 DSP BOARD

Michael Morrow, Boise State University, United States; Cameron Wright, University of Wyoming, United States; Thad Welch,

Boise State University, United States

SPED-P1.2: VERSATILE AND PORTABLE DSP PLATFORM FOR LEARNING EMBEDDED 2888 SIGNAL PROCESSING

Woon-Seng Gan, Abhishek Seth, School of EEE/NTU, Singapore; Sen M Kuo, Northern Illinois University, United States

SPED-P1.3: FPGA IMPLEMENTATION MADE EASY FOR APPLIED DIGITAL SIGNAL 2892 PROCESSING COURSES

Nasser Kehtarnavaz, Sidharth Mahotra, The University of Texas at Dallas, United States

SPED-P1.4: IN-CLASS DEMONSTRATIONS WITH A PORTABLE LABORATORY FOR 2896 TEACHING DSP TO COMPUTER ENGINEERING MAJORS

Andres Kwasinski, Rochester Institute of Technology, United States

SPED-P1.5: DSP EVOLUTION FROM A TEACHING POINT OF VIEW 2900

Naim Dahnoun, University of Bristol, United Kingdom; Jason Brand, Texas Instruments Inc., United Kingdom

SPED-P1.6: COLLABORATIVE SYSTEM FOR SIGNAL PROCESSING EDUCATION

Gregory Krudysz, James McClellan, Georgia Institute of Technology, United States

SPED-P1.7: POLYPHASE FILTERS - A MODEL FOR TEACHING THE ART OF 2908 DISCOVERY IN DSP

Mark Fowler, Binghamton University, United States

\section{SPCOM-L1: COMPRESSIVE SAMPLING AND SPARSE RECONSTRUCTION}

SPCOM-L1.1: EIGENSPACE SPARSITY FOR COMPRESSION AND DENOISING 2912

Ioannis Schizas, Georgios B. Giannakis, University of Minnesota, United States

SPCOM-L1.2: BASIS PURSUIT IN SENSOR NETWORKS

João Mota, Carnegie Mellon University / Institute of Systems and Robotics, United States; João Xavier, Pedro Aguiar, Institute of Systems and Robotics, Portugal; Markus Püschel, ETH Zürich, Switzerland

SPCOM-L1.3: ESTIMATING SPARSE MIMO CHANNELS HAVING COMMON SUPPORT 2920

Yann Barbotin, Ali Hormati, Ecole Polytechnique Fédérale de Lausanne, Switzerland; Sundeep Rangan, Polytechnic Institute of New York University, United States; Martin Vetterli, Ecole Polytechnique Fédérale de Lausanne, Switzerland

SPCOM-L1.4: APPLYING CSISZAR'S I-DIVERGENCE TO BLIND SPARSE CHANNEL 2924 ESTIMATION

Feng Wan, Urbashi Mitra, University of Southern California, United States

SPCOM-L1.5: COMPRESSIVE TRACKING OF DOUBLY SELECTIVE CHANNELS IN 2928 MULTICARRIER SYSTEMS BASED ON SEQUENTIAL DELAY-DOPPLER SPARSITY

Daniel Eiwen, University of Vienna, Austria; Georg Tauböck, Franz Hlawatsch, Vienna University of Technology, Austria; Hans Georg Feichtinger, University of Vienna, Austria 


\section{SPCOM-L2: SPECTRUM SENSING FOR COGNITIVE RADIO}

SPCOM-L2.1: DETECTION DIVERSITY OF MULTIANTENNA SPECTRUM SENSORS 2936

Gonzalo Vazquez-Vilar, Roberto Lopez-Valcarce, University of Vigo, Spain; Ashish Pandharipande, Philips Research,

Netherlands

SPCOM-L2.2: THE NON-BAYESIAN RESTLESS MULTI-ARMED BANDIT: A CASE OF 2940 NEAR-LOGARITHMIC REGRET

Wenhan Dai, Tsinghua University, China; Yi Gai, Bhaskar Krishnamachari, University of Southern California, United States;

Qing Zhao, University of California Davis, United States

SPCOM-L2.3: ON AUTOCORRELATION-BASED MULTIANTENNA SPECTRUM SENSING 2944 FOR COGNITIVE RADIOS IN UNKNOWN NOISE

Jitendra Tugnait, Auburn University, United States

SPCOM-L2.4: MULTIANTENNA DETECTION UNDER NOISE UNCERTAINTY AND 2948 PRIMARY USER'S SPATIAL STRUCTURE

David Ramirez, University of Cantabria, Spain; Gonzalo Vazquez-Vilar, Roberto Lopez-Valcarce, University of Vigo, Spain; Javier Via, Ignacio Santamaría, University of Cantabria, Spain

SPCOM-L2.5: TONE DETECTION OF NON-UNIFORMLY UNDERSAMPLED SIGNALS 2952 WITH FREQUENCY EXCISION

André Bourdoux, Sofie Pollin, Antoine Dejonghe, Liesbet Van der Perre, IMEC, Belgium

SPCOM-L2.6: A UNIFIED FRAMEWORK FOR GLRT-BASED SPECTRUM SENSING OF 2956 SIGNALS WITH COVARIANCE MATRICES WITH KNOWN EIGENVALUE MULTIPLICITIES

Erik Axell, Erik G. Larsson, Linköping University, Sweden

\section{SPCOM-L3: RESOURCE ALLOCATION AND GAME THEORY}

SPCOM-L3.1: NON-CONVEX UTILITY MAXIMIZATION IN GAUSSIAN MISO BROADCAST 2960 AND INTERFERENCE CHANNELS

Marco Rossi, New Jersey Institute of Technology, United States; Antonia Maria Tulino, Bell Laboratories (Alcatel-Lucent), United States; Osvaldo Simeone, Alexander M. Haimovich, New Jersey Institute of Technology, United States

SPCOM-L3.2: STOCHASTIC ANALYSIS OF TWO-TIER NETWORKS: EFFECT OF SPECTRUM ALLOCATION

Wang Chi Cheung, Tony Quee Seng Quek, Agency of Science, Technology And Research, Singapore; Marios Kountouris, Supélec, France

SPCOM-L3.3: DISTRIBUTED MULTIACCESS IN HIERARCHICAL COGNITIVE RADIO NETWORKS

Shiyao Chen, Lang Tong, Cornell University, United States

SPCOM-L3.4: NEW RESULTS ON ADAPTIVE COMPUTATIONAL RESOURCE ALLOCATION 2972 IN SOFT MIMO DETECTION

Mirsad Cirkic, Daniel Persson, Erik G. Larsson, Linköping University, Sweden

SPCOM-L3.5: JOINT BANDWIDTH AND POWER ALLOCATION IN COGNITIVE RADIO 2976 NETWORKS UNDER FADING CHANNELS

Xiaowen Gong, Sergiy Vorobyov, Chintha Tellambura, University of Alberta, Canada 


\section{SPCOM-L4: COOPERATIVE SPECTRUM SENSING}

SPCOM-L4.1: BEP WALLS FOR COLLABORATIVE SPECTRUM SENSING.

2984

Sachin Chaudhari, Jarmo Lunden, Visa Koivunen, Aalto University, Finland

SPCOM-L4.2: COOPERATIVE SENSING WITH SEQUENTIAL ORDERED

2988 TRANSMISSIONS TO SECONDARY FUSION CENTER

Laila Hesham, Ahmed Sultan, Mohammed Nafie, Nile University, Egypt; Fadel Digham, National Telecom Regulatory Authority, Egypt

SPCOM-L4.3: BASIS PURSUIT FOR SPECTRUM CARTOGRAPHY

2992

Juan Andrés Bazerque, Gonzalo Mateos, Georgios B. Giannakis, University of Minnesota, United States

SPCOM-L4.4: DECENTRALIZED SUPPORT DETECTION OF MULTIPLE MEASUREMENT

VECTORS WITH JOINT SPARSITY

Qing Ling, University of Science and Technology of China, China; Zhi Tian, Michigan Technological University, United States

SPCOM-L4.5: COOPERATIVE SPECTRUM SENSING BASED ON MATRIX RANK 3000 MINIMIZATION

Yue Wang, Beijing University of Posts and Telecommunications, China; Zhi Tian, Michigan Technological University, United States; Chunyan Feng, Beijing University of Posts and Telecommunications, China

SPCOM-L4.6: COOPERATIVE SENSING IN COGNITIVE NETWORKS UNDER 3004 MALICIOUS ATTACK

Mai Abdelhakim, Lei Zhang, Jian Ren, Tongtong Li, Michigan State University, United States

\section{SPCOM-L5: DISTRIBUTED AND COOPERATIVE PROCESSING}

SPCOM-L5.1: ASYMPTOTIC PERFORMANCE OF DISTRIBUTED DETECTION OVER 3008 RANDOM NETWORKS

Dragana Bajovic, Dusan Jakovetic, Instituto Superior Tecnico / Carnegie Mellon University, United States; João Xavier, Instituto Superior Tecnico / Lisbon, Portugal; Bruno Sinopoli, José M.F. Moura, Carnegie Mellon University, United States

SPCOM-L5.2: ROBUST DISTRIBUTED DETECTION, LOCALIZATION, AND ESTIMATION 3012 OF A DIFFUSIVE TARGET IN CLUSTERED WIRELESS SENSOR NETWORKS

Sami Aldalahmeh, Mounir Ghogho, University of Leeds, United Kingdom

SPCOM-L5.3: QUANTIZATION AND POWER ALLOCATION IN WIRELESS SENSOR 3016 NETWORKS WITH CORRELATED DATA

Muhammad Hafeez Chaudhary, Luc Vandendorpe, Catholic University of Louvain, Belgium

SPCOM-L5.4: SYSTEM-THEORETIC FORMULATION AND ANALYSIS OF DYNAMIC 3020 CONSENSUS PROPAGATION

Valentin Schwarz, Gerald Matz, University of Technology Vienna, Austria

SPCOM-L5.5: DOWNLINK MULTICELL COOPERATIVE TRANSMISSION WITH 3024 IMPERFECT CSI SHARING

Shengqian Han, Chenyang Yang, Beihang University, China

SPCOM-L5.6: OPTIMIZED EDGE APPEARANCE PROBABILITY FOR COOPERATIVE 3028 LOCALIZATION BASED ON TREE-REWEIGHTED NONPARAMETRIC BELIEF PROPAGATION Vladimir Savic, Universidad Politecnica de Madrid, Spain; Henk Wymeersch, Chalmers University of Technology, Sweden; Federico Penna, Politecnico di Torino, Italy; Santiago Zazo, Universidad Politecnica de Madrid, Spain 
SPCOM-L6.1: CONVEX APPROXIMATION ALGORITHMS FOR BACK-PRESSURE POWER 3032 CONTROL OF WIRELESS MULTI-HOP NETWORKS

Evaggelia Matskani, Nikolaos Sidiropoulos, Technical University of Crete, Greece; Leandros Tassiulas, University of Thessaly, Greece

SPCOM-L6.2: DISTRIBUTED ROUTING IN NETWORKS USING AFFINITY 3036 PROPAGATION

Manohar Shamaiah, Sang Hyun Lee, Sriram Vishwanath, Haris Vikalo, The University of Texas at Austin, United States

SPCOM-L6.3: SIMULTANEOUS SDR OPTIMALITY VIA A JOINT MATRIX 3040 DECOMPOSITION

Yuval Kochman, Massachusetts Institute of Technology, United States; Anatoly Khina, Uri Erez, Tel Aviv University, Israel

SPCOM-L6.4: ANALOG JOINT SOURCE-CHANNEL MULTIPLE DESCRIPTION CODING 3044 SCHEME OVER AWGN PARALLEL CHANNELS

Aitor Erdozain, Pedro M. Crespo, CEIT and TECNUN, Spain; Baltasar Beferull-Lozano, Universidad de Valencia, Spain

SPCOM-L6.5: PRACTICAL CODES FOR LOSSY COMPRESSION WHEN SIDE 3048 INFORMATION MAY BE ABSENT

Sivagnanasundaram Ramanan, John Walsh, Drexel University, United States

SPCOM-L6.6: FROM MAXIMUM LIKELIHOOD TO ITERATIVE DECODING 3052

Florence Alberge, Ziad Naja, University Paris-Sud, France; Pierre Duhamel, CNRS, France

\section{SPCOM-L7: MULTIUSER AND NETWORK MIMO}

SPCOM-L7.1: ON OPTIMAL CHANNEL TRAINING FOR UPLINK NETWORK MIMO 3056 SYSTEMS

Jakob Hoydis, Mari Kobayashi, Mérouane Debbah, Supélec, France

SPCOM-L7.2: AN ITERATIVELY WEIGHTED MMSE APPROACH TO DISTRIBUTED 3060 SUM-UTILITY MAXIMIZATION FOR A MIMO INTERFERING BROADCAST CHANNEL Qingjiang Shi, Shanghai Jiao Tong University, China; Meisam Razaviyayn, Zhi-Quan Luo, University of Minnesota, United States; Chen He, Shanghai Jiao Tong University, China

SPCOM-L7.3: TRANSCEIVER OPTIMIZATION FOR MULTI-USER MULTI-ANTENNA 3064 TWO-WAY RELAY CHANNELS

Can Sun, Chenyang Yang, Beihang University, China; Yonghui Li, Branka Vucetic, University of Sydney, Australia

SPCOM-L7.4: ON OPTIMAL PRECODING IN WIRELESS MULTICAST SYSTEMS 3068

Yiyue Wu, Haipeng Zheng, Robert Calderbank, Sanjeev R. Kulkarni, H. Vincent Poor, Princeton University, United States

SPCOM-L7.5: CHANNEL QUANTIZATION DESIGN IN MULTIUSER MIMO SYSTEMS: 3072 ASYMPTOTIC VERSUS PRACTICAL CONCLUSIONS

Emil Björnson, Konstantinos Ntontin, Björn Ottersten, KTH - Royal Institute of Technology, Sweden

SPCOM-L7.6: GRASSMANNIAN PREDICTIVE CODING FOR LIMITED FEEDBACK 3076 MULTIUSER MIMO SYSTEMS

Takao Inoue, National Instruments, United States; Robert W. Heath Jr., The University of Texas at Austin, United States

\section{SPCOM-L8: BEAMFORMING AND MIMO}

SPCOM-L8.1: PROBABILISTIC SINR CONSTRAINED ROBUST TRANSMIT 3080 BEAMFORMING: A BERNSTEIN-TYPE INEQUALITY BASED CONSERVATIVE APPROACH

Kun-Yu Wang, Tsung-Hui Chang, National Tsing Hua University, Taiwan; Wing-Kin Ma, Anthony Man-Cho So, The Chinese University of Hong Kong, Hong Kong SAR of China; Chong-Yung Chi, National Tsing Hua University, Taiwan 
SPCOM-L8.2: A LAGRANGIAN DUAL RELAXATION APPROACH TO ML MIMO DETECTION: 3084 REINTERPRETING REGULARIZED LATTICE DECODING

Jiaxian Pan, Wing-Kin Ma, The Chinese University of Hong Kong, Hong Kong SAR of China

SPCOM-L8.3: STOCHASTIC OPTIMIZATION BASED ON THE LAPLACE TRANSFORM 3088 ORDER WITH APPLICATIONS TO PRECODER DESIGNS

Minhua Ding, Keith Q. T. Zhang, City University of Hong Kong, Hong Kong SAR of China

SPCOM-L8.4: LINEAR PRECODING FOR TIME-VARYING MIMO CHANNELS WITH 3092 LOW-COMPLEXITY RECEIVERS

Jun Tong, Peter Schreier, Steven Weller, University of Newcastle, Australia; Louis Scharf, Colorado State University, United States

SPCOM-L8.5: ROBUST SINR-CONSTRAINED MISO DOWNLINK BEAMFORMING: 3096 WHEN IS SEMIDEFINITE PROGRAMMING RELAXATION TIGHT?

Enbin Song, Sichuan University, China; Qingjiang Shi, Shanghai Jiao Tong University, China; Maziar Sanjabi, Ruoyu Sun, ZhiQuan Luo, University of Minnesota, United States

SPCOM-L8.6: WHEN TO ADD ANOTHER DIMENSION WHEN COMMUNICATING OVER 3100 MIMO CHANNELS

Sreechakra Goparaju, Robert Calderbank, Princeton University, United States; William Carson, Miguel R. D. Rodrigues, University of Porto, Portugal; Fernando Pérez-Cruz, University Carlos III in Madrid, Spain

\section{SPCOM-P1: CAPACITY, NETWORKING, AND CODING}

SPCOM-P1.1: SHANNON MEETS NYQUIST: CAPACITY LIMITS OF SAMPLED ANALOG 3104 CHANNELS

Yuxin Chen, Stanford University, United States; Yonina C. Eldar, Technion / Israel Institute of Technology, Israel; Andrea Goldsmith, Stanford University, United States

SPCOM-P1.2: ON THE SUCCESS OF NETWORK INFERENCE USING A MARKOV 3108 ROUTING MODEL

Laura Balzano, Robert Nowak, University of Wisconsin Madison, United States; Matthew Roughan, University of Adelaide, Australia

SPCOM-P1.3: MAX-MIN FAIR RATE CONTROL BASED ON A SADDLE-POINT 3112 CHARACTERIZATION OF SOME PERRON ROOTS

Slawomir Stanczak, Michal Kaliszan, Fraunhofer German-Sino Lab for Mobile Communications, Germany; Mario Goldenbaum, TU Berlin, Germany

SPCOM-P1.4: SOCIAL NORM BASED INCENTIVE MECHANISMS FOR PEER-TO-PEER 3116 NETWORKS

Yu Zhang, Jaeok Park, Mihaela van der Schaar, University of California Los Angeles, United States

SPCOM-P1.5: COLLISION RESOLUTION IN MULTIPLE ACCESS NETWORKS WITH 3120 PHYSICAL-LAYER NETWORK CODING AND DISTRIBUTED FOUNTAIN CODING Giuseppe Cocco, Christian Ibars, Deniz Gündüz, Centre Tecnològic de Telecomunicacions de Catalunya (CTTC), Spain; Oscar del Rio Herrero, European Space Agency / ESTEC, Netherlands

SPCOM-P1.6: OPTIMAL WIRELESS NETWORKS BASED ON LOCAL CHANNEL STATE 3124 INFORMATION

Yichuan Hu, Alejandro Ribeiro, University of Pennsylvania, United States

SPCOM-P1.7: FOURIER DOMAIN DECODING ALGORITHM OF NON-BINARY LDPC 3128 CODES FOR PARALLEL IMPLEMENTATION

Kenta Kasai, Kohichi Sakaniwa, Tokyo Institute of Technology, Japan 
Andreas Winkelbauer, Gerald Matz, Vienna University of Technology, Austria

SPCOM-P1.9: PERFECT ROOT-OF-UNITY CODES WITH PRIME-SIZE ALPHABET

Mojtaba Soltanalian, Petre Stoica, Uppsala University, Sweden

SPCOM-P1.10: 2D MARKOVIAN SS CODES FLATTEN TIME-FREQUENCY DISTRIBUTION 3140 OF SIGNALS IN ASYNCHRONOUS GABOR DIVISION CDMA SYSTEMS

Tohru Kohda, Yutaka Jitsumatsu, Kyushu University, Japan; Kazuyuki Aihara, The University of Tokyo, Japan

\section{SPCOM-P2: SOURCE AND CHANNEL CODING}

SPCOM-P2.1: DESIGN OF UEP-BASED MSE-MINIMIZING RATELESS CODES FOR 3144 SOURCE-CHANNEL CODING

Amirpasha Shirazinia, Lei Bao, Mikael Skoglund, KTH - Royal Institute of Technology, Sweden

SPCOM-P2.2: ANALOG JOINT SOURCE-CHANNEL CODING IN RAYLEIGH FADING CHANNELS

Glauber Brante, Richard Souza, Federal University of Technology - Paraná, Brazil; Javier Garcia-Frias, University of Delaware, United States

SPCOM-P2.3: A NEW TRELLIS REPRESENTATION FOR SOURCE-CHANNEL RATE 3152 ALLOCATION

Romain Tajan, Charly Poulliat, Rodrigue Imad, Inbar Fijalkow, ETIS / CNRS/ENSEA/UCP, France

SPCOM-P2.4: JOINT SOURCE-CHANNEL-NETWORK CODING FOR BIDIRECTIONAL 3156 WIRELESS RELAYS

Francois Luus, Bodhaswar Maharaj, University of Pretoria, South Africa

SPCOM-P2.5: LOW-COMPLEXITY DETECTION OF GOLDEN CODES IN LDPC-CODED 3160 OFDM SYSTEMS

Iker Sobrón, Maitane Barrenechea, Pello Ochandiano, Lorena Martínez, Mikel Mendicute, Jon Altuna, University of Mondragon, Spain

SPCOM-P2.6: NONBINARY LDPC DECODING BY MIN-SUM WITH ADAPTIVE MESSAGE 3164 CONTROL

Weiguo Tang, Jie Huang, Lei Wang, Shengli Zhou, University of Connecticut, United States

SPCOM-P2.7: EFFICIENT ITERATIVE RECEIVER FOR BIT-INTERLEAVED CODED 3168 MODULATION ACCORDING TO THE DVB-T2 STANDARD

Meng Li, Charbel Abdel Nour, TELECOM Bretagne, France; Christophe Jego, L'ENSEIRB-MATMECA, France; Jianxiao Yang, Catherine Douillard, TELECOM Bretagne, France

SPCOM-P2.8: LINEAR TIME DECODING OF REAL-FIELD CODES OVER HIGH ERROR 3172 RATE CHANNELS

Zaixing He, Takahiro Ogawa, Miki Haseyama, Hokkaido University, Japan

SPCOM-P2.9: QUANTIZER DESIGN WITH MISMATCHED SIDE INFORMATION AT THE 3176 DECODER

Sepideh Shamaei, Mehrdad Valipour, University of Tehran, Iran

SPCOM-P2.10: RANDOM BLOCK-ANGULAR MATRICES FOR DISTRIBUTED DATA 3180 STORAGE

Paulo Ferreira, Bruno Jesus, José Vieira, Armando Pinho, University of Aveiro, Portugal 
SPCOM-P3.1: OPTIMAL TRANSMISSION STRATEGIES FOR CHANNEL CAPTURE

MITIGATION IN COGNITIVE RADIO NETWORKS

Yingxi Liu, Nikhil Kundargi, Ahmed Tewfik, The University of Texas at Austin, United States

SPCOM-P3.2: RESOURCE ALLOCATION FOR OFDMA COGNITIVE RADIOS UNDER 3188 CHANNEL UNCERTAINTY

Seung-Jun Kim, University of Minnesota, United States; Nasim Soltani, University of Tehran, Iran; Georgios B. Giannakis,

University of Minnesota, United States

SPCOM-P3.3: POWER ALLOCATION OPTIMIZATION IN OFDM-BASED COGNITIVE 3192 RADIOS BASED ON SENSING INFORMATION

Xiaoge Huang, Baltasar Beferull-Lozano, Universidad de Valencia, Spain

SPCOM-P3.4: STOCHASTIC RESOURCE ALLOCATION FOR COGNITIVE RADIO 3196 NETWORKS BASED ON IMPERFECT STATE INFORMATION

Antonio Marques, Rey Juan Carlos University, Spain; Georgios B. Giannakis, University of Minnesota, United States; Luis Lopez-Ramos, Javier Ramos, Rey Juan Carlos University, Spain

SPCOM-P3.5: DYNAMIC SPECTRUM MANAGEMENT IN DSL WITH ASYNCHRONOUS 3200 CROSSTALK

Rodrigo Moraes, Paschalis Tsiaflakis, Marc Moonen, Katholieke Universiteit Leuven, Belgium

SPCOM-P3.6: DESIGN OF DIGITAL PREDISTORTERS FOR WIDEBAND POWER 3204 AMPLIFIERS IN COMMUNICATION SYSTEMS WITH DYNAMIC SPECTRUM ALLOCATION Sungho Choi, Korea Advanced Institute of Science and Technology, Republic of Korea; Eui-Rim Jeong, Hanbat National University, Republic of Korea; Yong Hoon Lee, Korea Advanced Institute of Science and Technology, Republic of Korea

SPCOM-P3.7: GAME-THEORETIC RESOURCE ALLOCATION IN RELAY-ASSISTED 3208 DS/CDMA SYSTEMS WITH SUCCESSIVE INTERFERENCE CANCELLATION

Alessio Zappone, University of Cassino, Italy; Eduard Jorswieck, Dresden University of Technology, Germany

SPCOM-P3.8: OPTIMAL RADIO ACCESS IN FEMTOCELL NETWORKS BASED ON 3212 MARKOV MODELING OF INTERFERERS' ACTIVITY

Sergio Barbarossa, Alessandro Carfagna, Stefania Sardellitti, Marco Omilipo, Loreto Pescosolido, University of Rome La Sapienza, Italy

SPCOM-P3.9: CONVERGENCE OF THE ITERATIVE WATER-FILLING ALGORITHM 3216 WITH SEQUENTIAL UPDATES IN SPECTRUM SHARING SCENARIOS

Bhavani Shankar M. R, University of Luxembourg, Luxembourg; Peter von Wrycza, Mats Bengtsson, KTH - Royal Institute of Technology, Sweden; Björn Ottersten, University of Luxembourg, Luxembourg

SPCOM-P3.10: RATE CONTROL FOR PSD LIMITED MULTIPLE ACCESS SYSTEMS 3220 THROUGH LINEAR PROGRAMMING

Amir Leshem, Ephraim Zehavi, Bar-Ilan University, Israel

\section{SPCOM-P4: MIMO COMMUNICATIONS}

SPCOM-P4.1: ON THE ERGODIC CAPACITY OF JOINTLY-CORRELATED RICIAN FADING 3224 MIMO CHANNELS

Chao-Kai Wen, National Sun Yat-sen University, Taiwan; Shi Jin, Southeast University, China; Kai-Kit Wong, University College London, United Kingdom; Jung-Chieh Chen, National Kaohsiung Normal University, Taiwan; Pangan Ting, Industrial Technology Research Institute, Taiwan

SPCOM-P4.2: ADAPTIVE MIMO DETECTION ALGORITHM BY JOINTLY EXPLOITING 3228 THE PROPERTIES OF SIGNAL AND CHANNEL

Yuehua Ding, Yide Wang, Jean-Francois Diouris, Universite de Nantes, France 
SPCOM-P4.3: GAUSSIAN APPROXIMATION OF THE LLR DISTRIBUTION FOR THE ML 3232 AND PARTIAL MARGINALIZATION MIMO DETECTORS

Mirsad Cirkic, Daniel Persson, Erik G. Larsson, Jan-Ake Larsson, Linköping University, Sweden

SPCOM-P4.4: ON THE IMPLEMENTATION OF MIMO-OFDM SCHEMES USING 3236

PERTURBATION OF THE QR DECOMPOSITION: APPLICATION TO 3GPP LTE-A SYSTEMS

Sébastien Aubert, ST-ERICSSON, France; Jane Tournois, Vienna University of Technology, Austria; Fabienne Nouvel, INSA

IETR, France

SPCOM-P4.5: JOINT DATA DETECTION AND DOMINANT SINGULAR MODE ESTIMATION 3240 IN TIME VARYING RECIPROCAL MIMO SYSTEMS

Ranjitha Prasad, Bharath Bettagere Nagaraja, Chandra Ramabhadra Murthy, Indian Institute of Science, India

SPCOM-P4.6: ON THE SUM RATE OF ZF DETECTORS OVER CORRELATED K FADING 3244 MIMO CHANNELS

Michail Matthaiou, Chalmers University of Technology, Sweden; Nestor D. Chatzidiamantis, George K. Karagiannidis, Aristotle University of Thessaloniki, Greece

SPCOM-P4.7: ON SPATIO-TEMPORAL TOMLINSON HARASHIMA PRECODING IN IIR 3248 CHANNELS: MMSE SOLUTION, PROPERTIES, AND FAST COMPUTATION

Sander Wahls, Holger Boche, Technische Universität München, Germany

SPCOM-P4.8: FUNDAMENTAL DIVERSITY, MULTIPLEXING, AND ARRAY GAIN 3252 TRADEOFF UNDER DIFFERENT MIMO CHANNEL MODELS Luis G. Ordóñez, Universitat Politècnica de Catalunya - Barcelona Tech, Spain; Daniel P. Palomar, The Hong Kong University of Science and Technology, Hong Kong SAR of China; Javier R. Fonollosa, Universitat Politècnica de Catalunya - Barcelona Tech, Spain

SPCOM-P4.9: CHEAP SEMIDEFINITE RELAXATION MIMO DETECTION USING 3256 ROW-BY-ROW BLOCK COORDINATE DESCENT

Hoi-To Wai, Wing-Kin Ma, Anthony Man-Cho So, The Chinese University of Hong Kong, Hong Kong SAR of China

SPCOM-P4.10: SPATIAL AND TEMPORAL POWER ADAPTATION FOR SPACE-TIME CODED 3260 MIMO SYSTEMS WITH IMPERFECT CSI

Quan Kuang, Shu-Hung Leung, City University of Hong Kong, Hong Kong SAR of China; Xiangbin Yu, Nanjing University of Aeronautics and Astronautics, China

\section{SPCOM/SAM-P5: MIMO AND SENSOR NETWORKS}

SPCOM/SAM-P5.1: LINEAR IIR-MMSE PRECODING FOR FREQUENCY SELECTIVE 3264 MIMO CHANNELS

Sander Wahls, Holger Boche, Technische Universität München, Germany

SPCOM/SAM-P5.2: JOINT TRANSMITTER AND RECEIVER DESIGN WITH ADAPTIVE 3268 BEAMFORMING IN MIMO SC-FDMA SYSTEMS

Gang Xiong, Shalinee Kishore, Lehigh University, United States

SPCOM/SAM-P5.3: DESIGN OPTIMIZATION OF LINEAR PRECODERS FOR COMPLEX 3272 VECTOR GAUSSIAN CHANNELS WITH FINITE ALPHABET INPUTS

Chengshan Xiao, Yahong Zheng, Missouri University of Science and Technology, United States; Zhi Ding, University of California, United States

SPCOM/SAM-P5.4: FIXED- VERSUS FLOATING-POINT IMPLEMENTATION OF 3276 MIMO-OFDM DETECTOR

Janne Janhunen, University of Oulu, Finland; Perttu Salmela, Tampere University of Technology, Finland; Olli Silvén, Markku Juntti, University of Oulu, Finland 
DETECTION NECESSARY?

Brian Gestner, Xiaoli Ma, David V. Anderson, Georgia Institute of Technology, United States

SPCOM/SAM-P5.6: TIME-BASED LOCALIZATION FOR ASYNCHRONOUS WIRELESS

SENSOR NETWORKS

Yiyin Wang, Geert Leus, Delft University of Technology, Netherlands; Xiaoli Ma, Georgia Institute of Technology, United States

SPCOM/SAM-P5.7: DISTRIBUTED LINEAR DISCRIMINANT ANALYSIS

3288

Sergio Valcarcel Macua, Pavle Belanovic, Santiago Zazo, Universidad Politécnica de Madrid, Spain

SPCOM/SAM-P5.8: A GENERAL PROOF OF CONVERGENCE FOR ADAPTIVE

DISTRIBUTED BEAMFORMING SCHEMES

Chang-Ching Chen, Chia-Shiang Tseng, Che Lin, National Tsing Hua University, Taiwan

SPCOM/SAM-P5.9: DISTRIBUTED AUXILIARY PARTICLE FILTERS USING SELECTIVE 3296 GOSSIP

Deniz Üstebay, Mark Coates, Michael Rabbat, McGill University, Canada

SPCOM/SAM-P5.10: REACHING CONSENSUS IN ASYNCHRONOUS WSNS: ALGEBRAIC 3300 APPROACH

Ondrej Sluciak, Markus Rupp, Vienna University of Technology, Austria

\section{SPCOM-P6: RELAY COMMUNICATION NETWORKS}

SPCOM-P6.1: A FAST ALGORITHM FOR BEAMFORMING PROBLEMS IN DISTRIBUTED 3304 COMMUNICATION OF RELAY NETWORKS

Cong Sun, Yaxiang Yuan, Chinese Academy of Sciences, China

SPCOM-P6.2: FAIRNESS AND THROUGHPUT ENHANCING USER-COMBINING SCHEME 3308 BASED ON SUPERPOSITION CODING FOR A WIRELESS RELAY SYSTEM

Megumi Kaneko, Kazunori Hayashi, Hideaki Sakai, Kyoto University, Japan

SPCOM-P6.3: OPTIMAL AND LOW-COMPLEXITY ITERATIVE JOINT 3312 NETWORK/CHANNEL DECODING FOR THE MULTIPLE-ACCESS RELAY CHANNEL Xuan-Thang Vu, Marco Di Renzo, Pierre Duhamel, Laboratory of Signals and Systems (L2S) - CNRS - SUPELEC - Univ. ParisSud XI, France

SPCOM-P6.4: TRAINING DESIGN IN SINGLE RELAY AF COOPERATIVE SYSTEMS WITH 3316 CORRELATED CHANNELS

Christos Mavrokefalidis, University of Patras, Greece; Athanasios A. Rontogiannis, National Observatory of Athens, Greece; Kostas Berberidis, University of Patras, Greece

SPCOM-P6.5: ANALYZING THE PERFORMANCE OF ERROR-PRONE RELAY NETWORKS 3320

Sebastian Vorköper, Volker Kühn, University of Rostock, Germany

SPCOM-P6.6: SINGLE-SYMBOL DECODABLE DISTRIBUTED STBC FOR TWO-PATH 3324 SUCCESSIVE RELAY NETWORKS

Long Shi, Wei Zhang, University of New South Wales, Australia; Pak-Chung Ching, The Chinese University of Hong Kong, Hong Kong SAR of China

SPCOM-P6.7: DISTRIBUTED BEAMFORMING FOR OFDM-BASED COOPERATIVE RELAY 3328 NETWORKS UNDER TOTAL AND PER-RELAY POWER CONSTRAINTS

Wenjing Cheng, Qinfei Huang, National University of Defense Technology, China; Mounir Ghogho, University of Leeds, United Kingdom; Dongtang Ma, Jibo Wei, National University of Defense Technology, China

SPCOM-P6.8: NETWORK-MIMO BACKHAULING FOR QOS-CONSTRAINED RELAY 3332 TRANSMISSION

Josep Vidal, Adrian Agustin, Sandra Lagen, Eduard Valera, Olga Muñoz, Universitat Politècnica de Catalunya, Spain; Ana Garcia Armada, Matilde Sanchez Fernandez, Universidad Carlos III de Madrid, Spain 
Lennart Gerdes, Wolfgang Utschick, Technische Universität München, Germany

SPCOM-P6.10: DISTRIBUTED ONE BIT FEEDBACK EXTENDED ORTHOGONAL SPACE 3340 TIME CODING BASED ON SELECTION OF CYCLIC ROTATION FOR COOPERATIVE RELAY NETWORKS

Abdulghani Elazreg, Jonathon A. Chambers, Loughborough University, United Kingdom

\section{SPCOM-P7: INTERFERENCE ALIGNMENT AND MULTIUSER MIMO}

SPCOM-P7.1: INTERFERENCE ALIGNMENT IN SINGLE-BEAM MIMO NETWORKS VIA 3344 HOMOTOPY CONTINUATION

Oscar Gonzalez, Ignacio Santamaría, University of Cantabria, Spain

SPCOM-P7.2: INTERFERENCE ALIGNMENT IN CLUSTERED AD HOC NETWORKS: 3348 HIGH RELIABILITY REGIME AND PER-CLUSTER ALOHA

Roland Tresch, Telecommunications Research Center Vienna, Austria; Giusi Alfano, Politecnico di Torino, Italy; Maxime Guillaud, Vienna University of Technology, Austria

SPCOM-P7.3: INTERFERENCE SELF-MITIGATING BEAMFORMING FOR THE K-USER 3352 MIMO IC

Jianqi Wang, Amitav Mukherjee, Lee Swindlehurst, University of California Irvine, United States

SPCOM-P7.4: INTERFERENCE ALIGNMENT IN MIMO CELLULAR NETWORKS

Binnan Zhuang, Randall Berry, Michael Honig, Northwestern University, United States

SPCOM-P7.5: USER ADMISSION IN MIMO INTERFERENCE ALIGNMENT NETWORKS

Behrang Nosrat-Makouei, Jeffrey G. Andrews, Robert W. Heath Jr., The University of Texas at Austin, United States

SPCOM-P7.6: ADAPTIVE BEAM TRACKING FOR INTERFERENCE ALIGNMENT IN TIME-VARYING MIMO INTERFERENCE CHANNELS: CONJUGATE GRADIENT APPROACH

Junse Lee, Heejung Yu, Youngchul Sung, Yong Hoon Lee, Korea Advanced Institute of Science and Technology, Republic of Korea

SPCOM-P7.7: A CONVEX APPROXIMATION APPROACH TO WEIGHTED SUM RATE 3368 MAXIMIZATION OF MULTIUSER MISO INTERFERENCE CHANNEL UNDER OUTAGE CONSTRAINTS

Wei-Chiang Li, Tsung-Hui Chang, Che Lin, Chong-Yung Chi, National Tsing Hua University, Taiwan

SPCOM-P7.8: CLOSED-FORM PARAMETERIZATION OF THE PARETO BOUNDARY FOR 3372 THE TWO-USER MISO INTERFERENCE CHANNEL

Johannes Lindblom, Eleftherios Karipidis, Erik G. Larsson, Linköping University, Sweden

SPCOM-P7.9: MULTICAST TRANSMIT BEAMFORMING USING A RANDOMIZE-IN-TIME 3376 STRATEGY

Xiaoxiao Wu, Wing-Kin Ma, The Chinese University of Hong Kong, Hong Kong SAR of China

SPCOM-P7.10: USER SELECTION SCHEMES FOR MAXIMIZING THROUGHPUT OF 3380 MULTIUSER MIMO SYSTEMS USING ZERO FORCING BEAMFORMING Anh Nguyen, Bhaskar D. Rao, University of California San Diego, United States

\section{SPCOM-P8: CHANNEL EQUALIZATION AND FEEDBACK}

SPCOM-P8.1: SINGLE-CARRIER SYSTEMS WITH MMSE LINEAR EQUALIZERS: 3384 PERFORMANCE DEGRADATION DUE TO CHANNEL AND CFO ESTIMATION ERRORS Athanasios Liavas, Despoina Tsipouridou, Technical University of Crete, Greece 
SPCOM-P8.4: GENERALIZED GEOMETRIC MEAN DECOMPOSITION AND DFE MMSE

TRANSCEIVER DESIGN FOR CYCLIC PREFIX SYSTEMS

Chih-Hao Liu, Palghat P. Vaidyanathan, California Institute of Technology, United States

SPCOM-P8.5: ITERATIVE FDE FOR ASYNCHRONOUS SINGLE-CARRIER MULTIUSER 3400 SYSTEMS

Wei Han, Qinye Yin, Ang Feng, Xi'an Jiaotong University, China

SPCOM-P8.6: EFFICIENT LATTICE-REDUCTION-AIDED MMSE DECISION-FEEDBACK 3404 EQUALIZATION

Robert Fischer, Universität Erlangen-Nürnberg, Germany

SPCOM-P8.7: PREDICTIVE VECTOR QUANTIZATION FOR WIRELESS TRANSMITTER 3408 ADAPTATION WITH LIMITED FEEDBACK

Danda B. Rawat, Dusadee Treeumnuk, Dimitrie C. Popescu, Old Dominion University, United States

SPCOM-P8.8: ITERATIVE WATER FILLING BASED ON SLNR WITH 1-SHOT 1-BIT 3412 FEEDBACK

Kazunori Hayashi, Megumi Kaneko, Takeshi Fujii, Hideaki Sakai, Kyoto University, Japan; Yoji Okada, Sumitomo Electric Industries, Ltd., Japan

SPCOM-P8.9: HOW MUCH FEEDBACK OVERHEAD IS REQUIRED FOR BASE STATION 3416 COOPERATIVE TRANSMISSION TO OUTPERFORM NON-COOPERATIVE TRANSMISSION?

Xueying Hou, Chenyang Yang, Beihang University, China

SPCOM-P8.10: STOCHASTIC TRANSCEIVER DESIGN IN MULTI-ANTENNA CHANNELS 3420 WITH STATISTICAL CHANNEL STATE INFORMATION

Andreas Gründinger, Michael Joham, Wolfgang Utschick, Technische Universität München, Germany

\section{SPCOM-P9: SECRECY AND COMMUNICATIONS}

SPCOM-P9.1: COOPERATION STRATEGIES FOR SECRECY IN MIMO RELAY 3424 NETWORKS WITH UNKNOWN EAVESDROPPER CSI

Jing Huang, A. Lee Swindlehurst, University of California Irvine, United States

SPCOM-P9.2: A GAME THEORETIC APPROACH TO EAVESDROPPER COOPERATION IN 3428 MISO WIRELESS NETWORKS

Joohyun Peter Cho, University of Southern California, United States; Y.-W. Peter Hong, National Tsing Hua University, Taiwan; C. -C. Jay Kuo, University of Southern California, United States

SPCOM-P9.3: CHARACTERIZING PHYSICAL-LAYER SECRECY WITH UNKNOWN 3432 EAVESDROPPER LOCATIONS AND CHANNELS

Mounir Ghogho, University of Leeds, United Kingdom; Ananthram Swami, Army Research Lab, United States

SPCOM-P9.4: A ROBUST ARTIFICIAL NOISE AIDED TRANSMIT DESIGN FOR MISO 3436 SECRECY

Qiang Li, Wing-Kin Ma, The Chinese University of Hong Kong, China

SPCOM-P9.5: FILTER DESIGN WITH SECRECY CONSTRAINTS: THE 3440

MULTIPLE-INPUT MULTIPLE-OUTPUT GAUSSIAN WIRETAP CHANNEL WITH ZERO FORCING RECEIVE FILTERS

Hugo Reboredo, Vinay Prabhu, Miguel R. D. Rodrigues, Instituto de Telecomunicações / University of Porto, Portugal; João Xavier, Instituto de Sistemas e Robótica / Instituto Superior Técnico, Portugal 
SPCOM-P9.7: MIXED-INTEGER LINEAR PROGRAMMING FRAMEWORK FOR MAX-MIN 3448 POWER CONTROL WITH SINGLE-STAGE INTERFERENCE CANCELLATION

Eleftherios Karipidis, Di Yuan, Erik G. Larsson, Linköping University, Sweden

SPCOM-P9.8: REINFORCEMENT LEARNING FOR ENERGY-EFFICIENT WIRELESS 3452 TRANSMISSION

Nicholas Mastronarde, Mihaela van der Schaar, University of California Los Angeles, United States

SPCOM-P9.9: FIXED POINT ITERATION FOR MAX-MIN SIR BALANCING WITH 3456 GENERAL INTERFERENCE FUNCTIONS

Nikola Vucic, Martin Schubert, Fraunhofer German-Sino Lab for Mobile Communications, Germany

SPCOM-P9.10: JOINT BLIND ESTIMATION OF CARRIER PHASE AND FREQUENCY 3460 OFFSET FOR QAM SIGNALS USING CIRCULAR HARMONIC DECOMPOSITION

Alexander Sergienko, Alexander Petrov, Electrotechnical University, Russian Federation

\section{SPCOM-P10: CHANNEL ESTIMATION}

SPCOM-P10.1: ANALYSIS OF THE PILOT CONTAMINATION EFFECT IN VERY LARGE 3464 MULTICELL MULTIUSER MIMO SYSTEMS FOR PHYSICAL CHANNEL MODELS

Hien Ngo, Linköping University, Sweden; Thomas Marzetta, Bell Laboratories, United States; Erik G. Larsson, Linköping University, Sweden

SPCOM-P10.2: ERROR-ENTROPY BASED CHANNEL STATE ESTIMATION OF SPATIALLY 3468 CORRELATED MIMO-OFDM

Hoang Tuan, Ha Kha, University of New South Wales, Australia; Ha Nguyen, University of Saskatchewan, Canada

SPCOM-P10.3: ESTIMATION OF SPACE-TIME VARYING CHANNELS USING SIGNAL 3472 SUBSPACE PROJECTION AND SOFT INFORMATION

Shu Cai, Xidian University, China; Matsumoto Tad, University of Oulu, Finland; Kehu Yang, Xidian University, China

SPCOM-P10.4: CONTINUOUS PILOT BASED ADAPTIVE ESTIMATION FOR IDMA 3476 SYSTEMS ON UNDERWATER ACOUSTIC CHANNELS

Salah Al-iesawi, Charalampos Tsimenidis, Bayan Sharif, Martin Johnston, Newcastle University, United Kingdom

SPCOM-P10.5: SIMPLIFIED EM CHANNEL ESTIMATION IN LTE SYSTEMS 3480

Yang Liu, Serdar Sezginer, Sequans Communications, France

SPCOM-P10.6: DIRECTION-RESOLVED ESTIMATION OF MULTIPATH PARAMETERS FOR 3484 UWB CHANNELS: A PARTIALLY COLLAPSED GIBBS SAMPLER METHOD

Georg Kail, Vienna University of Technology, Austria; Klaus Witrisal, Graz University of Technology, Austria; Franz, Hlawatsch, Vienna University of Technology, Austria

SPCOM-P10.7: A HYBRID COMPRESSED SENSING ALGORITHM FOR SPARSE CHANNEL 3488 ESTIMATION IN MIMO OFDM SYSTEMS

Chenhao Qi, Lenan Wu, Southeast University, China

SPCOM-P10.8: JOINT ESTIMATION OF CHANNEL AND CARRIER FREQUENCY OFFSET 3492 FROM THE EMITTER, IN AN UPLINK OFDMA SYSTEM Babar Aziz, ETIS / ENSEA / Unversité de Cergy-Pontoise, France; Inbar Fijalkow, Myriam Ariaudo, ETIS / ENSEA / Unversité de Cergy-Pontoise / CNRS, France

SPCOM-P10.9: MIXTURE KALMAN FILTERING FOR JOINT CARRIER RECOVERY AND 3496 CHANNEL ESTIMATION IN TIME-SELECTIVE RAYLEIGH FADING CHANNELS

Ali Nasir, Salman Durrani, Rodney A. Kennedy, Australian Natinoal University, Australia

SPCOM-P10.10: DATA AIDED PHASE TRACKING AND SYMBOL DETECTION FOR CPM IN 3500 FREQUENCY-FLAT FADING CHANNEL

Wenwen Wang, Saman S. Abeysekera, Nanyang Technological University, Singapore 
SPCOM-P11.1: SMOOTHING FIR FILTERING OF DISCRETE STATE-SPACE POLYNOMIAL

SIGNAL MODELS

Oscar Ibarra-Manzano, Yuriy Shmaliy, Guanajuato University, Mexico; Luis Morales-Mendoza, Veracruz University, Mexico

SPCOM-P11.2: TIMING ADJUSTMENT TECHNIQUES TO MITIGATE INTERFERENCE 3508 BETWEEN MULTIPLE NODES IN OFDMA MESH NETWORKS

Sungeun Lee, Xiaoli Ma, Georgia Institute of Technology, United States

SPCOM-P11.3: A ROBUST CLOCK SYNCHRONIZATION ALGORITHM FOR WIRELESS 3512 SENSOR NETWORKS

Jang-sub Kim, Jaehan Lee, Erchin Serpedin, Khalid Qaraqe, Texas A\&M University, United States

SPCOM-P11.4: ANALYSIS OF NON-COHERENT CODE TRACKING FOR NPSK SYSTEMS 3516 IN PRESENCE OF NOISE AND FADING

Ramin Vali, Stevan M. Berber, The University of Auckland, New Zealand

SPCOM-P11.5: TIME DOMAIN SYNCHRONIZATION AND DECODING OF P1 SYMBOL IN 3520 DVB-T2

Mingchao Yu, Parastoo Sadeghi, Australian National University, Australia

SPCOM-P11.6: OPTIMUM CHIP PULSE SHAPE DESIGN FOR TIMING SYNCHRONIZATION

Felix Antreich, German Aerospace Center (DLR), Germany; Josef A. Nossek, Munich University of Technology, Germany

SPCOM-P11.7: RSS-BASED NODE LOCALIZATION IN THE PRESENCE OF ATTENUATING

\section{OBJECTS}

Andrea Edelstein, Xi Chen, McGill University, Canada; Yunpeng Li, Beijing University of Posts and Telecommunications, China;

Michael Rabbat, McGill University, Canada

SPCOM-P11.8: SEMI-DEFINITE PROGRAMMING FOR DISTRIBUTED TRACKING OF 3532 DYNAMIC OBJECTS BY NONLINEAR SENSOR NETWORK

Umar Rashid, Tuan Hoang, Ha Kha, The University of New South Wales, Australia; Ha Nguyen, University of Saskatchewan, Canada

SPCOM-P11.9: ON SYNTHESIZING CROSS AMBIGUITY FUNCTIONS 3536

Hao He, University of Florida, United States; Petre Stoica, Uppsala University, Sweden; Jian Li, University of Florida, United States

SPCOM-P11.10: IMPACT OF THE CORRELATION BETWEEN FORWARD AND 3540 BACKSCATTER CHANNELS ON RFID SYSTEM PERFORMANCE

Chen He, Z. Jane Wang, University of British Columbia, Canada

SPCOM-P12: MULTICARRIER COMMUNICATIONS AND OFDM

SPCOM-P12.1: REDUCED COMPLEXITY BLIND DETERMINISTIC FREQUENCY 3544 OFFSET ESTIMATION IN OFDM SYSTEMS

Hyoung-Goo Jeon, Dongeui University, Republic of Korea; Kyoung-Soo Kim, Electronics and Telecommunications Research Institute, Republic of Korea; Erchin Serpedin, Texas A\&M University, United States

SPCOM-P12.2: IMPULSIVE INTERFERENCE MITIGATION IN AD HOC NETWORKS 3548 BASED ON ALPHA-STABLE MODELING AND PARTICLE FILTERING

Nouha Jaoua, Emmanuel Duflos, Philippe Vanheeghe, Ecole Centrale de Lille, France; Laurent Clavier, François Septier, Telecom Lille 1, France

SPCOM-P12.3: ROBUST FREQUENCY SYNCHRONIZATION FOR AN OFDMA UPLINK 3552 SYSTEM DISTURBED BY A COGNITIVE RADIO SYSTEM INTERFERENCE

Hector Poveda, Guillaume Ferre, Eric Grivel, Universite Bordeaux 1 - IPB - ENSEIRB-MATMECA, France 
TIME-VARYING CHANNELS

Tao Xu, Geert Leus, Delft University of Technology, Netherlands; Urbashi Mitra, University of Southern California, United

States

SPCOM-P12.5: INTERFERENCE CANCELLATION FOR OFDM SYSTEMS WITH 3560 HIERARCHICAL MODULATION OVER NON-LINEAR SATELLITE CHANNELS

Emad Al-Dalakta, Charalampos Tsimenidis, Bayan Sharif, Arafat Al-Dweik, Newcastle University, United Kingdom

SPCOM-P12.6: PILOT OPTIMIZATION FOR TIME-DELAY AND CHANNEL ESTIMATION IN 3564 OFDM SYSTEMS

Michael Larsen, Raytheon Company, United States; Gonzalo Seco-Granados, Universitat Autonoma de Barcelona, Spain; A. Lee Swindlehurst, University of California Irvine, United States

SPCOM-P12.7: OFO ESTIMATION METHODS WITH WIDE ACQUISITION RANGES FOR 3568 MB-OFDM-BASED UWB SYSTEMS

Lin Bai, Qinye Yin, Xi'an Jiaotong University, China

SPCOM-P12.8: GENERALIZED INTERIOR-POINT METHOD FOR CONSTRAINED PEAK 3572 POWER MINIMIZATION OF OFDM SIGNALS

Zhenhua Yu, Georgia Institute of Technology, United States; Robert J. Baxley, Georgia Tech Research Institute, United States; G. Tong Zhou, Georgia Institute of Technology, United States

SPCOM-P12.9: BLIND PERIODICALLY TIME-VARYING MMOE CHANNEL SHORTENING 3576 FOR OFDM SYSTEMS

Donatella Darsena, Parthenope University, Italy; Giacinto Gelli, Luigi Paura, Francesco Verde, University Federico II, Italy

SPCOM-P12.10: HOW MANY KNOWN SYMBOLS ARE REQUIRED FOR LINEAR 3580 CHANNEL ESTIMATION IN OFDM?

Shuichi Ohno, Emmanuel Manasseh, Masayoshi Nakamoto, Hiroshima University, Japan

\section{SPTM-L1: NON-STATIONARY SIGNAL ANALYSIS}

SPTM-L1.1: A MULTIWINDOW TIME-FREQUENCY APPROACH BASED ON THE 3584 CONCEPTS OF ROBUST ESTIMATE THEORY

Irena Orovic, Nikola Zaric, Srdjan Stankovic, University of Montenegro, Montenegro; Moeness G. Amin, University of Villanova, United States

SPTM-L1.2: CONSTRUCTION OF POSITIVE TIME-FREQUENCY DISTRIBUTIONS 3588 USING DYNAMIC COPULA

Shwan Ashrafi, University of New Mexico, United States; Hamidreza Amindavar, Amirkabir University of Technology, Iran; James A. Ritcey, University of Washington, United States; Rodney Lynn Kirlin, University of Victoria, United States

SPTM-L1.3: THE ASYMPTOTIC PROPERTIES OF POLYNOMIAL PHASE ESTIMATION BY 3592 LEAST SQUARES PHASE UNWRAPPING

Robby McKilliam, Vaughan Clarkson, University of Queensland, Australia; Barry Quinn, Macquarie University, Australia; Bill Moran, University of Melbourne, Australia

SPTM-L1.4: MAP-BASED ESTIMATION OF THE PARAMETERS OF NON-STATIONARY 3596 GAUSSIAN PROCESSES FROM NOISY OBSERVATIONS

Alexander Krueger, Reinhold Haeb-Umbach, University of Paderborn, Germany

SPTM-L1.5: TRANSITIONAL SURROGATES. 3600

Pierre Borgnat, Patrick Flandrin, CNRS - ENS Lyon, France; André Ferrari, Cédric Richard, Université de Nice SophiaAntipolis, France 

AND ITS APPLICATION

Wen-Liang Hsue, Chung Yuan Christian University, Taiwan; Soo-Chang Pei, Jian-Jiun Ding, National Taiwan University, Taiwan

\section{SPTM-L2: DETECTION THEORY AND METHODS}

SPTM-L2.1: ROBUST CHANGEPOINT DETECTION BASED ON MULTIVARIATE RANK 3608 STATISTICS

Alexandre Lung-Yut-Fong, Céline Lévy-Leduc, Olivier Cappé, Institut Telecom \& CNRS / LTCI / Telecom ParisTech, France

SPTM-L2.2: SCALABLE ROBUST HYPOTHESIS TESTS USING GRAPHICAL MODELS .

Divyanshu Vats, Carnegie Mellon University, United States; Vishal Monga, Umamahesh Srinivas, The Pennsylvania State

University, United States; José M.F. Moura, Carnegie Mellon University, United States

SPTM-L2.3: A NEW CRITERION FOR OPTIMAL CONSTRAINED MINIMAX DETECTION 3616 AND CLASSIFICATION

Lionel Fillatre, Igor Nikiforov, Université de Technologie de Troyes, France

SPTM-L2.4: A ROBUST ESTIMATOR AND DETECTOR OF CIRCULARITY OF COMPLEX 3620

SIGNALS

Esa Ollila, Visa Koivunen, Aalto University, Finland; H. Vincent Poor, Princeton University, United States

SPTM-L2.5: DETECTION OF GEOMETRICALLY KNOWN TARGETS IN 3624 THROUGH-THE-WALL RADAR IMAGING

Christian Debes, Abdelhak M. Zoubir, Technische Universität Darmstadt, Germany; Moeness G. Amin, Villanova University, United States

SPTM-L2.6: A BAYESIAN MARKED POINT PROCESS FOR OBJECT DETECTION. 3628 APPLICATION TO MUSE HYPERSPECTRAL DATA

Florent Chatelain, Aude Costard, Olivier Michel, GIPSA-Lab / UMR 5216-CNRS / University of Grenoble, France

\section{SPTM-L3: TARGET DETECTION AND LOCALISATION}

SPTM-L3.1: MULTI-SENSOR PHD: CONSTRUCTION AND IMPLEMENTATION BY SPACE 3632 PARTITIONING

Emmanuel Delande, CNRS, France; Emmanuel Duflos, Philippe Vanheeghe, Ecole Centrale de Lille, France; Dominique Heurguier, Thales Communications, France

SPTM-L3.2: A KALMAN-LIKE ALGORITHM WITH NO REQUIREMENTS FOR NOISE AND 3636 INITIAL CONDITIONS

Yuriy Shmaliy, Guanajuato University, Mexico

SPTM-L3.3: URBAN TERRAIN TRACKING IN HIGH CLUTTER WITH 3640 WAVEFORM-AGILITY

Bhavana Chakraborty, Jun Zhang, Antonia Papandreou-Suppappola, Darryl Morrell, Arizona State University, United States

SPTM-L3.4: AN APPROXIMATE MINIMUM MOSPA ESTIMATOR

David Crouse, Peter Willett, University of Connecticut, United States; Marco Guerriero, Elt Elettronica S.p.A., Italy; Lennart Svensson, Chalmers University of Technology, Sweden 
ENVIRONMENT

Bentarage Sachintha Karunaratne, Mark Morelande, Bill Moran, The University of Melbourne, Australia; Stephen Howard,

Defence Science and Technology Organisation, Australia

\section{SPTM-L4: COMPRESSED SENSING: THEORY AND METHODS I}

SPTM-L4.1: THE VALUE OF REDUNDANT MEASUREMENT IN COMPRESSED SENSING 3656

Victoria Kostina, Princeton University, United States; Marco Duarte, Duke University, United States; Sina Jafarpour, Princeton University, United States; Robert Calderbank, Duke University, United States

SPTM-L4.2: COMPRESSIVE SENSING MEETS GAME THEORY 3660

Sina Jafarpour, Princeton University, United States; Volkan Cevher, Ecole Polytechnique Fédérale de Lausanne, Switzerland;

Robert Schapire, Princeton University, United States

SPTM-L4.3: LORENTZIAN BASED ITERATIVE HARD THRESHOLDING FOR 3664 COMPRESSED SENSING

Rafael Carrillo, Kenneth Barner, University of Delaware, United States

SPTM-L4.4: SPARSITY-UNDERSAMPLING TRADEOFF OF COMPRESSED SENSING IN 3668 THE COMPLEX DOMAIN

Zai Yang, Cishen Zhang, Nanyang Technological University, Singapore

SPTM-L4.5: SRF: MATRIX COMPLETION BASED ON SMOOTHED RANK FUNCTION.

Hooshang Ghasemi, Mohmmadreza Malek-Mohammadi, Massoud Babaie-Zadeh, Sharif University of Technology, Iran;

Christian Jutten, Institut Universitaire de France, France

SPTM-L4.6: GENERALIZED RESTRICTED ISOMETRY PROPERTY FOR ALPHA-STABLE 3676 RANDOM PROJECTIONS

Daniel Otero, Gonzalo R. Arce, University of Delaware, Colombia

\section{SPTM-L5: CLASSIFICATION AND PATTERN RECOGNITION}

SPTM-L5.1: SPATIALLY-CORRELATED SENSOR DISCRIMINANT ANALYSIS

Kush Varshney, IBM T.J. Watson Research Center, United States

SPTM-L5.2: ROTATION INVARIANT FEATURE EXTRACTION FROM 3-D ACCELERATION 3684 SIGNALS

Takumi Kobayashi, Koiti Hasida, Nobuyuki Otsu, National Institute of Advanced Industrial Science and Technology, Japan

SPTM-L5.3: THE GROUPED TWO-SIDED ORTHOGONAL PROCRUSTES PROBLEM

Bryan Conroy, Peter Ramadge, Princeton University, United States

SPTM-L5.4: DESTINATION-AWARE TARGET TRACKING VIA SYNTACTIC SIGNAL PROCESSING.

Mustafa Fanaswala, Vikram Krishnamurthy, University of British Columbia, Canada; Langford White, The University of Adelaide, Australia

SPTM-L5.5: JUMP FUNCTION KOLMOGOROV FOR OVERLAPPING AUDIO EVENT 3696 CLASSIFICATION

Huy Dat Tran, Haizhou Li, Institute for Infocomm Research / Agency of Science Technology And Research, Singapore 
SPTM-L6.1: EMPIRICAL WEIGHTING FOR BLIND SOURCE SEPARATION IN A

MULTIPLE-SNAPSHOTS SCENARIO

Arie Yeredor, Tel Aviv University, Israel

SPTM-L6.2: BLIND EXTRACTION OF IMPROPER QUATERNION SOURCES

3708

Soroush Javidi, Clive Cheong Took, Cyrus Jahanchahi, Imperial College London, United Kingdom; Nicolas Le Bihan, GIPSA-

Lab, France; Danilo P. Mandic, Imperial College London, United Kingdom

SPTM-L6.3: AN EXTENSION OF THE ICA MODEL USING LATENT VARIABLES

3712

Selwa Rafi, Marc Castella, Wojciech Pieczynski, Institut Telecom / Telecom SudParis, France

SPTM-L6.4: ROBUST UNDERDETERMINED BLIND AUDIO SOURCE SEPARATION OF 3716 SPARSE SIGNALS IN THE TIME-FREQUENCY DOMAIN

Si Mohamed Aziz Sbaï, Abdeldjalil Ä̈ssa-El-Bey, Dominique Pastor, Institut Télécom / Télécom Bretagne, France

SPTM-L6.5: ON THE RELATION BETWEEN ICA AND MMSE BASED SOURCE 3720 SEPARATION

Benedikt Loesch, Bin Yang, University of Stuttgart, Germany

SPTM-L6.6: MULTIDIMENSIONAL ICA AND ITS PERFORMANCE ANALYSIS APPLIED TO 3724 CMB OBSERVATIONS

Dana Lahat, Tel Aviv University, Israel; Jean-François Cardoso, Télécom ParisTech, France; Maude Le Jeune, Université Denis Diderot-Paris VII, France; Hagit Messer, Tel Aviv University, Israel

\section{SPTM-L7: COMPRESSED SENSING: THEORY AND METHODS II}

SPTM-L7.1: STATISTICAL COMPRESSIVE SENSING OF GAUSSIAN MIXTURE MODELS 3728

Guoshen Yu, Guillermo Sapiro, University of Minnesota, United States

SPTM-L7.2: COMPRESSED SENSING SIGNAL RECOVERY VIA A* ORTHOGONAL 3732 MATCHING PURSUIT

Nazim Burak Karahanoglu, TUBITAK - BILGEM, Turkey; Hakan Erdogan, Sabanci University, Turkey

SPTM-L7.3: WEIGHTED COMPRESSED SENSING AND RANK MINIMIZATION 3736

Samet Oymak, M. Amin Khajehnejad, Babak Hassibi, California Institute of Technology, United States

SPTM-L7.4: LOW-RANK MATRIX COMPLETION WITH GEOMETRIC PERFORMANCE 3740 GUARANTEES

Wei Dai, Ely Kerman, Olgica Milenkovic, University of Illinois Urbana-Champaign, United States

SPTM-L7.5: GUARANTEED ERROR CORRECTION BASED ON FOURIER COMPRESSIVE 3744 SENSING AND PROJECTIVE GEOMETRY

B.S. Adiga, Girish Chandra, Shreeniwas Sapre, Tata Consultancy Services, India

SPTM-L7.6: DETERMINISTIC COMPRESSED-SENSING MATRICES: WHERE TOEPLITZ 3748 MEETS GOLAY

Kezhi Li, Cong Ling, Imperial College London, United Kingdom; Lu Gan, Brunel University, United Kingdom

\section{SPTM-L8: DISTRIBUTED AND COLLABORATIVE SIGNAL PROCESSING}

SPTM-L8.1: COOPERATIVE PREY HERDING BASED ON DIFFUSION ADAPTATION 3752

Sheng-Yuan Tu, Ali H. Sayed, University of California Los Angeles, United States 


\section{CONSENSUS}

Ondrej Hlinka, Ondrej Sluciak, Franz, Hlawatsch, Vienna University of Technology, Austria; Petar Djuric, Stony Brook

University, United States; Markus Rupp, Vienna University of Technology, Austria

SPTM-L8.3: CONTENT PREFERENCE ESTIMATION IN ONLINE SOCIAL NETWORKS: 3760 MESSAGE PASSING VERSUS SPARSE RECONSTRUCTION ON GRAPHS

Jacob Chakareski, Ecole Polytechnique Fédérale de Lausanne, Switzerland

SPTM-L8.4: CONVERGENCE OF A DISTRIBUTED PARAMETER ESTIMATOR FOR 3764 SENSOR NETWORKS WITH LOCAL AVERAGING OF THE ESTIMATES

Pascal Bianchi, Gersende Fort, Walid Hachem, Jérémie Jakubowicz, LTCI Telecom ParisTech / CNRS, France

SPTM-L8.5: PERFORMANCE LIMITS OF LMS-BASED ADAPTIVE NETWORKS. 3768

Xiaochuan Zhao, Ali H. Sayed, University of California Los Angeles, United States

SPTM-L8.6: EFFICIENT DISTRIBUTED RESAMPLING FOR PARTICLE FILTERS 3772

Balakumar Balasingam, Miodrag Bolic, University of Ottawa, Canada; Petar Djuric, State University of New York, United States; Joaquin Miguez, Universidad Carlos III de Madrid, Spain

\section{SPTM-L9: ESTIMATION THEORY AND METHODS}

SPTM-L9.1: ASYMPTOTICALLY MMSE-OPTIMUM PILOT DESIGN FOR COMB-TYPE 3776 OFDM CHANNEL ESTIMATION IN HIGH-MOBILITY SCENARIOS

K. M. Zahidul Islam, The University of Texas at Dallas, United States; Tareq Y. Al-Naffouri, King Fahd University of Petroleum \& Minerals, Saudi Arabia; Naofal Al-Dhahir, The University of Texas at Dallas, United States

SPTM-L9.2: ROBUST BINARY LEAST SQUARES: RELAXATIONS AND ALGORITHMS. 3780

Efthymios Tsakonas, Joakim Jaldén, KTH - Royal Institute of Technology, Sweden; Björn Ottersten, University of Luxembourg,

Luxembourg

SPTM-L9.3: ESTIMATING PRINCIPAL COMPONENTS OF LARGE COVARIANCE 3784 MATRICES USING THE NYSTRÖM METHOD

Nicholas Arcolano, Patrick Wolfe, Harvard University, United States

SPTM-L9.4: COOPERATIVE MAXIMUM LIKELIHOOD ESTIMATION FOR FLUID FLOW 3788 DYNAMICS IN BIOSENSOR ARRAYS

Maryam Abolfath-Beygi, Vikram Krishnamurthy, University of British Columbia, Canada

SPTM-L9.5: WEIGHTED AND STRUCTURED SPARSE TOTAL LEAST-SQUARES FOR 3792 PERTURBED COMPRESSIVE SAMPLING

Hao Zhu, Georgios B. Giannakis, University of Minnesota, United States; Geert Leus, Delft University of Technology, Netherlands

SPTM-L9.6: PSEUDO MAXIMUM LIKELIHOOD ESTIMATIONS OF BALLISTIC MISSILE 3796 PRECESSION FREQUENCY

Lihua Liu, National University of Defence Technology, China; Mounir Ghogho, Des McLernon, University of Leeds, United Kingdom; Weidong Hu, National University of Defence Technology, China

\section{SPTM-P1: NON-STATIONARY SIGNALS AND TIME-VARYING SYSTEMS}

SPTM-P1.1: JOINT FREQUENCY SPECTRAL LAG REPRESENTATION FOR 3800 CROSS-FREQUENCY MODULATION ANALYSIS IN THE BRAIN

Ali Mutlu, Selin Aviyente, Michigan State University, United States 
SPTM-P1.3: EVOLUTIVE METHOD BASED ON A GENERALIZED EIGENVALUE

DECOMPOSITION TO ESTIMATE TIME VARYING AUTOREGRESSIVE PARAMETERS FROM

NOISY OBSERVATIONS

Hiroshi Ijima, Wakayama University, Japan; Julien Petitjean, Eric Grivel, Université de Bordeaux, France

SPTM-P1.4: A TIME-FREQUENCY METHOD FOR INCREASING THE SIGNAL-TO-NOISE 3812

RATIO IN SYSTEM IDENTIFICATION WITH EXPONENTIAL SWEEPS

Piotr Majdak, Peter Balazs, Wolfgang Kreuzer, Austrian Academy of Sciences, Austria; Monika Dörfler, University of Vienna, Austria

SPTM-P1.5: SIMULTANEOUS PROCESSING OF SOUND SOURCE SEPARATION AND 3816 MUSICAL INSTRUMENT IDENTIFICATION USING BAYESIAN SPECTRAL MODELING

Katsutoshi Itoyama, Kyoto University, Japan; Masataka Goto, National Institute of Advanced Industrial Science and Technology, Japan; Kazunori Komatani, Tetsuya Ogata, Hiroshi G. Okuno, Kyoto University, Japan

SPTM-P1.6: A NOVEL ANALYTICAL APPROACH TO ORTHOGONAL BASES EXTRACTION 3820 FROM AM-FM SIGNALS

Mohammadali Sebghati, Hamidreza Amindavar, Amirkabir University of Technology, Iran

SPTM-P1.7: RÉNYI INFORMATION MEASURES FOR SPECTRAL CHANGE DETECTION 3824 Marco Liuni, Universita`di Firenze / IRCAM - CNRS STMS Analysis/Synthesis Team, France; Axel Röbel, Institut de Recherche et Coordination Acoustique/Musique / CNRS STMS / Analysis/Synthesis Team, France; Marco Romito, Universita`di Firenze, Italy; Xavier Rodet, Institut de Recherche et Coordination Acoustique/Musique / CNRS STMS / Analysis/Synthesis Team, France

SPTM-P1.8: DUAL FRAME OF FREQUENCY WARPING OPERATORS 3828

Salvatore Caporale, Nicolò Speciale, University of Bologna, Italy

SPTM-P1.9: A NOVEL ALGORITHM OF SEEKING FRFT ORDER FOR SPEECH 3832 PROCESSING

DuoJia Ma, Xiang Xie, JingMing Kuang, Beijing Institute of Technology, China

SPTM-P1.10: SCALE-DEPENDENT ANALYSIS OF IONOSPHERE FLUCTUATIONS. 3836

Stephane Roux, Patrice Abry, Ecole Normale Superieure de Lyon, France; Petra Koucká Knižová, Zbyšek Mošna, Institute of Atmospheric Physics / Academy of Sciences of the Czech Republic, Czech Republic

\section{SPTM-P2: DETECTION AND ESTIMATION THEORY AND METHODS}

SPTM-P2.1: GENERALIZATIONS OF BLOM AND BLOEM'S PDF DECOMPOSITION FOR 3840 PERMUTATION-INVARIANT ESTIMATION

David Crouse, Peter Willett, Yaakov Bar-Shalom, University of Connecticut, United States

SPTM-P2.2: A TIME-DISTRIBUTED PHASE SPACE HISTOGRAM FOR DETECTING 3844 TRANSIENT SIGNALS

Florin-Marian Birleanu, Cornel Ioana, GIPSA-Lab, France; Alexandru Serbanescu, Military Technical Academy, Romania; Jocelyn Chanussot, GIPSA-Lab, France

SPTM-P2.3: COMPLEX RANDOM MATRICES AND MULTIPLE-ANTENNA SPECTRUM 3848 SENSING

Tharmalingam Ratnarajah, C. Zhong, A. Kortun, Mathini Sellathurai, Queen's University Belfast, United Kingdom; C.B. Papadias, Athens Information Technology, Greece

SPTM-P2.4: SMART GRID MONITORING FOR INTRUSION AND FAULT DETECTION 3852 WITH NEW LOCALLY OPTIMUM TESTING PROCEDURES

Qian He, Rick Blum, Lehigh University, United States 
SPTM-P2.5: RANK-DEFICIENT QUADRATIC-FORM MAXIMIZATION OVER M-PHASE 3856

ALPHABET: POLYNOMIAL-COMPLEXITY SOLVABILITY AND ALGORITHMIC

DEVELOPMENTS

Anastasios Kyrillidis, Ecole Polytechnique Fédérale de Lausanne, Switzerland; George Karystinos, Technical University of

Crete, Greece

SPTM-P2.6: MULTIPLE-CHANNEL DETECTION OF A GAUSSIAN TIME SERIES OVER 3860 FREQUENCY-FLAT CHANNELS

David Ramirez, Javier Via, Ignacio Santamaría, University of Cantabria, Spain; Louis Scharf, Colorado State University, United States

SPTM-P2.7: TIME-LAGGED DIRECTED INFORMATION 3864

Ying Liu, Selin Aviyente, Michigan State University, United States

SPTM-P2.8: DETECTION OF TARGETS EMBEDDED IN MULTIPATH CLUTTER WITH 3868 TIME REVERSAL

Nicholas O'Donoughue, Joel Harley, José M.F. Moura, Carnegie Mellon University, United States

SPTM-P2.9: MULTI-SENSOR ESTIMATION AND DETECTION OF PHASE-LOCKED

SINUSOIDS

Christoph Reller, Hans-Andrea Loeliger, Stefano Maranò, ETH Zürich, Switzerland

SPTM-P2.10: A REWEIGHTED LEAST SQUARES ALGORITHM FOR DETECTION OF QAM 3876 SIGNALING IN MIMO CHANNELS

Katsumi Konishi, Kogakuin University, Japan; Toshihiro Furukawa, Tokyo University of Science, Japan

\section{SPTM-P3: COMPRESSIVE SENSING AND SPARSITY I}

SPTM-P3.1: ROBUST NONPARAMETRIC REGRESSION BY CONTROLLING SPARSITY 3880

Gonzalo Mateos, Georgios B. Giannakis, University of Minnesota, United States

SPTM-P3.2: COMPRESSIVE POWER SPECTRAL DENSITY ESTIMATION

Michael Lexa, Michael Davies, University of Edinburgh, United Kingdom; Janosch Nikolic, Swiss Federal Institute of

Technology, Switzerland; John Thompson, University of Edinburgh, United Kingdom

SPTM-P3.3: ADAPTIVE COMPRESSIVE SENSING AND PROCESSING FOR RADAR 3888 TRACKING

Ioannis Kyriakides, University of Nicosia, Cyprus

SPTM-P3.4: SPARSE VARIABLE REDUCED RANK REGRESSION VIA STIEFEL OPTIMIZATION

Magnus Ulfarsson, University of Iceland, Iceland; Victor Solo, School of Electrical Engineering and Telecommunication, Australia

SPTM-P3.5: THE ROTATIONAL LASSO 3896

Alexander Lorbert, Peter Ramadge, Princeton University, United States

SPTM-P3.6: CAUSAL SIGNAL RECOVERY FROM U-INVARIANT SAMPLES 3900

Tomer Michaeli, Yonina C. Eldar, Technion / Israel Institute of Technology, Israel; Volker Pohl, Technical University Berlin, Germany

SPTM-P3.7: IMPROVED MODEL-BASED SPECTRAL COMPRESSIVE SENSING VIA 3904 NESTED LEAST SQUARES

Mahdi Shaghaghi, Sergiy Vorobyov, University of Alberta, Canada

SPTM-P3.8: ESTIMATION AND DYNAMIC UPDATING OF TIME-VARYING SIGNALS WITH 3908 SPARSE VARIATIONS

M. Salman Asif, Adam Charles, Justin Romberg, Christopher Rozell, Georgia Institute of Technology, United States 


\section{SPTM-P4: COMPRESSIVE SENSING AND SPARSITY II}

SPTM-P4.1: SLIDING WINDOW GREEDY RLS FOR SPARSE FILTERS 3916

Alexandru Onose, Bogdan Dumitrescu, Ioan Tabus, Tampere University of Technology, Finland

SPTM-P4.2: COMPRESSIVE SAMPLING WITH A SUCCESSIVE APPROXIMATION ADC 3920 ARCHITECTURE

Chenchi Luo, James McClellan, Georgia Institute of Technology, United States

SPTM-P4.3: COMPRESSED LEARNING OF HIGH-DIMENSIONAL SPARSE FUNCTIONS

Karin Schnass, Jan Vybiral, Austrian Academy of Sciences, Austria

SPTM-P4.5: ITERATIVE REWEIGHTED ALGORITHMS FOR SPARSE SIGNAL RECOVERY 3932 WITH TEMPORALLY CORRELATED SOURCE VECTORS

Zhilin Zhang, Bhaskar D. Rao, University of California San Diego, United States

SPTM-P4.6: ADJUGATE PAIRS OF SPARSE ARRAYS FOR SAMPLING TWO DIMENSIONAL 3936 SIGNALS

Palghat P. Vaidyanathan, Piya Pal, California Institute of Technology, United States

SPTM-P4.7: USING THE KERNEL TRICK IN COMPRESSIVE SENSING: ACCURATE 3940 SIGNAL RECOVERY FROM FEWER MEASUREMENTS

Hanchao Qi, Shannon Hughes, University of Colorado at Boulder, United States

SPTM-P4.8: SUB-NYQUIST SAMPLING OF SHORT PULSES 3944

Ewa Matusiak, University of Vienna, Austria; Yonina C. Eldar, Technion, Israel

SPTM-P4.9: BAYESIAN COMPRESSIVE SENSING FOR CLUSTERED SPARSE SIGNALS. 3948

Lei Yu, Hong Sun, Wuhan University, China; Jean-Pierre Barbot, Ecole Nationale Supérieure d'Electronique et de Ses

Applications, France; Gang Zheng, Institut national de recherche en informatique et automatique, France

SPTM-P4.10: TLS-FOCUSS FOR SPARSE RECOVERY WITH PERTURBED DICTIONARY 3952

Xuebing Han, Hao Zhang, Huadong Meng, Tsinghua University, China

\section{SPTM-P5: MONTE CARLO METHODS FOR SIGNAL PROCESSING}

SPTM-P5.1: MCMC INFERENCE OF THE SHAPE AND VARIABILITY OF TIME-RESPONSE 3956 SIGNALS

Dmitriy Katz-Rogozhnikov, Kush Varshney, Aleksandra Mojsilovic, Moninder Singh, IBM T.J. Watson Research Center, United States

SPTM-P5.2: A REVERSIBLE JUMP MCMC ALGORITHM FOR BAYESIAN CURVE FITTING 3960 BY USING SMOOTH TRANSITION REGRESSION MODELS Matthieu Sanquer, Florent Chatelain, GIPSA-lab / University of Grenoble, France; Mabrouka El-Guedri, Electricité de France, France; Nadine Martin, GIPSA-lab / University of Grenoble, France 
TRACKING

Onur Ozdemir, Andro Computational Solutions, United States; Ruixin Niu, Pramod K. Varshney, Syracuse University, United

States; Andrew L. Drozd, Andro Computational Solutions, United States

SPTM-P5.6: SEQUENTIAL MONTE CARLO RADIO-FREQUENCY TOMOGRAPHIC 3976 TRACKING

Yunpeng Li, Beijing University of Posts and Telecommunications, China; Xi Chen, Mark Coates, McGill University, Canada; Bo Yang, Beijing University of Posts and Telecommunications, China

SPTM-P5.7: THE COMPRESSIVE MULTIPLEXER FOR MULTI-CHANNEL 3980 COMPRESSIVE SENSING

John Slavinsky, Jason Laska, Rice University, United States; Mark Davenport, Stanford University, United States; Richard Baraniuk, Rice University, United States

SPTM-P5.8: A MODE PRESERVING PARTICLE FILTER . 3984

Mark Morelande, Alan Zhang, The University of Melbourne, Australia

SPTM-P5.9: PENALIZED L1 MINIMIZATION FOR RECONSTRUCTION OF 3988 TIME-VARYING SPARSE SIGNALS Wei Chen, University of Cambridge, United Kingdom; Miguel R. D. Rodrigues, University of Porto, Portugal; Ian Wassell, University of Cambridge, United Kingdom

SPTM-P5.10: OPTIMAL SIR ALGORITHM VS. FULLY ADAPTED AUXILIARY PARTICLE 3992 FILTER : A MATTER OF CONDITIONAL INDEPENDENCE

Francois Desbouvries, Yohan Petetin, Emmanuel Monfrini, Telecom SudParis, France

\section{SPTM-P6: SPARSITY, SAMPLING AND RECONSTRUCTION}

SPTM-P6.1: EXTENSION OF THE GLOBAL MATCHED FILTER TO STRUCTURED 3996 GROUPS OF ATOMS: APPLICATION TO HARMONIC SIGNALS.

Jean Jacques Fuchs, Université de Rennes 1, France

SPTM-P6.2: FREQUENCY DOMAIN COMPENSATION OF SPURIOUS SIDEBANDS IN A/D 4000 CIRCUITS

Shang-Kee Ting, Ali H. Sayed, University of California Los Angeles, United States

SPTM-P6.3: VARIATIONAL BAYESIAN KALMAN FILTERING IN DYNAMICAL TOMOGRAPHY 4004

Boujemaa Ait-El-Fquih, Thomas Rodet, Université Paris sud 11, France

SPTM-P6.4: COMPRESSIVE SENSING IN THROUGH-THE-WALL RADAR IMAGING

Michael Leigsnering, Christian Debes, Abdelhak M. Zoubir, Technische Universität Darmstadt, Germany

SPTM-P6.5: SHORT AND SMOOTH SAMPLING TRAJECTORIES FOR COMPRESSED 4012 SENSING

Rebecca Willett, Duke University, United States

SPTM-P6.6: SAMPLING AND RECONSTRUCTING DIFFUSION FIELDS WITH 4016 LOCALIZED SOURCES

Juri Ranieri, Amina Chebira, Yue M. Lu, Martin Vetterli, Ecole Polytechnique Fédérale de Lausanne, Switzerland

SPTM-P6.7: TRAJECTORY TRIANGULATION: 3D MOTION RECONSTRUCTION WITH L1 4020 OPTIMIZATION

Mingyu Chen, Ghassan AlRegib, Biing-Hwang(Fred) Juang, Georgia Institute of Technology, United States 
Saikat Chatterjee, Dennis Sundman, Mikael Skoglund, KTH - Royal Institute of Technology, Sweden

SPTM-P6.9: ITERATIVE HARD THRESHOLDING FOR COMPRESSED SENSING WITH 4028

PARTIALLY KNOWN SUPPORT

Rafael Carrillo, Luisa Polania, Kenneth Barner, University of Delaware, United States

SPTM-P6.10: BAYESIAN FRAMEWORK AND MESSAGE PASSING FOR JOINT SUPPORT 4032 AND SIGNAL RECOVERY OF APPROXIMATELY SPARSE SIGNALS

Shubha Shedthikere, Ananthanarayanan Chockalingam, Indian Institute of Science, India

\section{SPTM-P7: SIGNAL AND SYSTEM MODELING AND ESTIMATION I}

SPTM-P7.1: MIXED NORMS WITH OVERLAPPING GROUPS AS SIGNAL PRIORS 4036

Ilker Bayram, Istanbul Technical University, Turkey

SPTM-P7.2: NONNEGATIVE 3-WAY TENSOR FACTORIZATION VIA CONJUGATE 4040 GRADIENT WITH GLOBALLY OPTIMAL STEPSIZE

Jean-Philip Royer, Pierre Comon, I3S, France; Nadège Thirion-Moreau, ISITV LSEET, France

SPTM-P7.3: A CLUSTERING BASED FRAMEWORK FOR DICTIONARY BLOCK 4044 STRUCTURE IDENTIFICATION

Ender M. Eksioglu, Istanbul Technical University, Turkey

SPTM-P7.4: TARGET TRACKING AND LOCALIZATION WITH AMBIGUOUS PHASE 4048 MEASUREMENTS OF SENSOR NETWORKS

Yongqiang Cheng, National University of Defense Technology, China; Xuezhi Wang, University of Melbourne, Australia; Terry Caelli, National ICT Australia, Australia; Bill Moran, University of Melbourne, Australia

SPTM-P7.5: INTERPOLATION BASED ON STATIONARY AND ADAPTIVE AR(1) MODELING. 4052

Eija Johansson, Marie Ström, Mats Viberg, Lennart Svensson, Chalmers University of Technology, Sweden

SPTM-P7.6: IDENTIFICATION AND COMPENSATION OF WIENER-HAMMERSTEIN 4056 SYSTEMS WITH FEEDBACK

Andrew Bolstad, Benjamin Miller, MIT Lincoln Laboratory, United States; Joel Goodman, US Navy Research Laboratory, United States; James Vian, MIT Lincoln Laboratory, United States; Janani Kalyanam, University of California San Diego, United States

SPTM-P7.7: SPARSE DECOMPOSITION OF TRANSFORMATION-INVARIANT SIGNALS 4060 WITH CONTINUOUS BASIS PURSUIT

Chaitanya Ekanadham, Daniel Tranchina, New York University, United States; Eero Simoncelli, HHMI / New York University, United States

SPTM-P7.8: ENTROPY-CONSTRAINED QUANTIZATION OF EXPONENTIALLY DAMPED 4064 SINUSOIDS PARAMETERS

Olivier Derrien, CNRS LMA / Université de Toulon, France; Roland Badeau, Gaël Richard, Télécom ParisTech / CNRS LTCI,

France

SPTM-P7.9: PROPORTIONATE AFFINE PROJECTION SIGN ALGORITHMS FOR SPARSE 4068 SYSTEM IDENTIFICATION IN IMPULSIVE INTERFERENCE

Zengli Yang, Y. Rosa Zheng, Steve L. Grant, Missouri University of Science and Technology, United States

SPTM-P7.10: AUGMENTED COMPLEX MATRIX FACTORISATION 4072

David Looney, Danilo P. Mandic, Imperial College London, United Kingdom 
SPTM-P8.1: IDENTIFICATION OF ARMA MODELS USING INTERMITTENT AND 4076 QUANTIZED OUTPUT OBSERVATIONS

Damián Marelli, University of Newcastle, Australia; Keyou You, Nanyang Technological University, China; Minyue Fu, University of Newcastle, Australia

SPTM-P8.2: FAST ORTHOGONAL DECOMPOSITION OF VOLTERRA CUBIC KERNELS 4080 USING OBLIQUE UNFOLDING

Rémy Boyer, University of Paris XI (UPS), France; Roland Badeau, Télécom ParisTech, France; Gérard Favier, CNRS, France

SPTM-P8.3: IDENTIFICATION OF MISO NONLINEAR SYSTEMS VIA THE 4084 SEMIPARAMETRIC APPROACH

Jiaqing Lv, Miroslaw Pawlak, University of Manitoba, Canada

SPTM-P8.4: ON THE IDENTIFICATION OF PARAMETRIC UNDERSPREAD LINEAR 4088 SYSTEMS

Waheed U. Bajwa, Duke University, United States; Kfir Gedalyahu, Yonina C. Eldar, Technion / Israel Institute of Technology, Israel

SPTM-P8.5: ON SELECTING THE HYPERPARAMETERS OF THE DPM MODELS FOR 4092 THE DENSITY ESTIMATION OF OBSERVATION ERRORS

Asma Rabaoui, LAGIS / Ecole Centrale de lille, France; Nicolas Viandier, Juliette Marais, LEOST / INRETS, France; Emmanuel Duflos, LAGIS / Ecole Centrale de lille, France

SPTM-P8.6: ROBUST MODEL ORDER SELECTION FOR CORNEAL HEIGHT DATA BASED 4096 ON $\mathrm{ESTIMATION}$

Michael Muma, Abdelhak M. Zoubir, Technische Universität Darmstadt, Germany

SPTM-P8.7: ENHANCED POISSON SUM REPRESENTATION FOR ALPHA-STABLE 4100 PROCESSES

Tatjana Lemke, Simon J. Godsill, University of Cambridge, United Kingdom

SPTM-P8.8: SEMI-ALGEBRAIC CANONICAL DECOMPOSITION OF MULTI-WAY ARRAYS 4104 AND JOINT EIGENVALUE DECOMPOSITION

Xavier Luciani, Université de Nice Sophia-Antipolis, France; Laurent Albera, University of Rennes 1 and INSERM, France

SPTM-P8.9: AN UNIQUENESS CONDITION FOR THE 4-WAY CANDECOMP/PARAFAC 4108 MODEL WITH COLLINEAR LOADINGS IN THREE MODES

David Brie, Sebastian Miron, Fabrice Caland, Centre de Recherche en Automatique de Nancy, France; Christian Mustin, Laboratoire des Interactions Microorganismes-Mineraux-Matiere Organique dans les Sols, France

SPTM-P8.10: AN UNSUPERVISED ALGORITHM FOR HYBRID/MORPHOLOGICAL SIGNAL 4112 DECOMPOSITION

Matthieu Kowalski, Thomas Rodet, University Paris-Sud, France

\section{SPTM-P9: ADAPTIVE FILTER ANALYSIS AND DESIGN}

SPTM-P9.1: STOCHASTIC BEHAVIOR ANALYSIS OF THE GAUSSIAN KERNEL LEAST 4116 MEAN SQUARE ALGORITHM

Wemerson D. Parreira, José C. M. Bermudez, Federal University of Santa Catarina, Brazil; Cédric Richard, Nice SophiaAntipolis University, France; Jean-Yves Tourneret, University of Toulouse, France

SPTM-P9.2: STEADY-STATE ANALYSIS OF THE NLMS ALGORITHM WITH REUSING 4120 COEFFICIENT VECTOR AND A METHOD FOR IMPROVING ITS PERFORMANCE

Seong-Eun Kim, Jae-Woo Lee, Woo-Jin Song, Pohang University of Science and Technology, Republic of Korea 
SPTM-P9.3: PROPORTIONATE-TYPE NORMALIZED LEAST MEAN SQUARE ALGORITHM 4124 WITH GAIN ALLOCATION MOTIVATED BY MINIMIZATION OF MEAN-SQUARE-WEIGHT DEVIATION FOR COLORED INPUT

Kevin Wagner, Naval Research Laboratory, United States; Milos Doroslovacki, The George Washington University, United States

SPTM-P9.4: TRACKING PERFORMANCE OF ADAPTIVELY BIASED ADAPTIVE FILTERS. 4128 Jerónimo Arenas-García, Universidad Carlos III de Madrid, Spain; Miguel Lázaro-Gredilla, Universidad de Cantabria, Spain

SPTM-P9.5: INCREMENTAL-COOPERATIVE STRATEGIES IN COMBINATION OF 4132 ADAPTIVE FILTERS

Wilder Lopes, Cassio Lopes, University of Sao Paulo, Brazil

SPTM-P9.6: ON GRADIENT TYPE ADAPTIVE FILTERS WITH NON-SYMMETRIC MATRIX 4136 STEP-SIZES

Markus Rupp, Vienna University of Technology, Austria

SPTM-P9.7: EVALUATION OF ADAPTIVE BLIND SIMO IDENTIFICATION IN TERMS OF A 4140 NORMALIZED FILTER-PROJECTION MISALIGNMENT

Dominic Schmid, Gerald Enzner, Ruhr-Universität Bochum, Germany

SPTM-P9.8: A COMPLETE ENSEMBLE EMPIRICAL MODE DECOMPOSITION WITH 4144 ADAPTIVE NOISE

Maria-E Torres, Marcelo A. Colominas, Gastón Schlotthauer, Universidad Nacional de Entre Rios, Argentina; Patrick Flandrin, Ecole Normale Supérieure de Lyon, France

SPTM-P9.9: EXPLICIT RECURSIVITY INTO REPRODUCING KERNEL HILBERT 4148 SPACES

Devis Tuia, Gustavo Camps-Valls, Image Processing Laboratory, Spain; Manel Martínez-Ramón, Department Signal Theory and Communications, Spain

SPTM-P9.10: THE LEAST-MEAN-MAGNITUDE-PHASE ALGORITHM WITH APPLICATIONS 4152 TO COMMUNICATIONS SYSTEMS

Scott Douglas, LGT Corporation / Southern Methodist University, United States; Danilo P. Mandic, Imperial College London, United Kingdom

\section{SPTM-P10: PERFORMANCE ANALYSIS AND BOUNDS}

SPTM-P10.1: PERFORMANCE BOUNDS FOR SPARSE PARAMETRIC COVARIANCE 4156 ESTIMATION IN GAUSSIAN MODELS

Alexander Jung, Vienna University of Technology, Austria; Sebastian Schmutzhard, University of Vienna, Austria; Franz Hlawatsch, Vienna University of Technology, Austria; Alfred O. Hero III, University of Michigan, United States

SPTM-P10.2: NOISE POWER GAIN AS A MEASURE OF ERRORS IN DISCRETE-TIME 4160 TRANSVERSAL ESTIMATORS

Yuriy Shmaliy, Oscar Ibarra-Manzano, Guanajuato University, Mexico

SPTM-P10.3: STABILITY OF CANDECOMP-PARAFAC TENSOR DECOMPOSITION

Petr Tichavský, Institute of Information Theory and Automation, Czech Republic; Zbynek Koldovsky, Technical University of Liberec, Czech Republic

SPTM-P10.4: INFORMATION-THEORETIC ANALYSIS OF DESYNCHRONIZATION 4168 INVARIANT OBJECT IDENTIFICATION

Oleksiy Koval, Sviatoslav Voloshynovskiy, Farzad Farhadzadeh, Taras Holotyak, Fokko Beekhof, University of Geneva, Switzerland 
SPTM-P10.6: COMPARATIVE THRESHOLD PERFORMANCE STUDY FOR CONDITIONAL 4176 AND UNCONDITIONAL DIRECTION-OF-ARRIVAL ESTIMATION

Yuri I. Abramovich, Defence Science and Technology Organisation, Australia; Ben A. Johnson, Lockheed Martin Australia, Australia

SPTM-P10.7: COMPACT SUPPORT KERNELS BASED TIME-FREQUENCY 4180 DISTRIBUTIONS: PERFORMANCE EVALUATION

Mansour Abed, Abdel Hamid Ibn Badis University, Algeria; Adel Belouchrani, Ecole Nationale Polytechnique (ENP), Algeria; Mohamed Cheriet, Synchromedia / University of Quebec, Canada; Boualem Boashash, Qatar University, Qatar

SPTM-P10.8: BIAS ANALYSIS OF THE FORCED CHOICE DETECTION TEST FOR 4184 RAYLEIGH/RICIAN STATISTICS

Graham Pulford, Thales Underwater Systems, France

SPTM-P10.9: PERFORMANCE ANALYSIS OF MDL CRITERION FOR THE DETECTION OF 4188 NONCIRCULAR OR/AND NONGAUSSIAN COMPONENTS

Jean-Pierre Delmas, Yann Meurisse, Telecom SudParis, France

SPTM-P10.10: IMPROVEMENT TO ESPRIT-TYPE FREQUENCY ESTIMATORS VIA 4192 REDUCING DATA REDUNDANCY

Weize Sun, H. C. So, City University of Hong Kong, Hong Kong SAR of China

\section{SPTM-P11: SAMPLING AND RECONSTRUCTION}

SPTM-P11.1: SIGNAL RECONSTRUCTION FROM SINE WAVE CROSSINGS 4196

Holger Boche, Ullrich Mönich, Technische Universität München, Germany

SPTM-P11.2: SIGNAL RECOVERY IN SHIFT-INVARIANT SPACES FROM PARTIAL 4200 FREQUENCY DATA

Volker Pohl, Technical University Berlin, Germany; Yonina C. Eldar, Technion / Israel Institute of Technology, Israel

SPTM-P11.3: GROUP TESTING MEETS TRAITOR TRACING.

Peter Meerwald, Teddy Furon, INRIA Rennes Bretagne Atlantique, France

SPTM-P11.4: DOWNSAMPLING GRAPHS USING SPECTRAL THEORY 4208

Sunil Kumar, Antonio Ortega, University of Southern California, United States

SPTM-P11.5: EFFICIENT MAXIMUM LIKELIHOOD ESTIMATION OF A 2-D COMPLEX 4212 SINUSOIDAL BASED ON BARYCENTRIC INTERPOLATION

Jesus Selva, University of Alicante, Spain

SPTM-P11.6: FOCUSS IS A CONVEX-CONCAVE PROCEDURE 4216

Md Mashud Hyder, Kaushik Mahata, University of Newcastle, Australia

SPTM-P11.7: EMPIRICAL DIVERGENCE MAXIMIZATION FOR QUANTIZER DESIGN: AN 4220 ANALYSIS OF APPROXIMATION ERROR

Michael Lexa, University of Edinburgh, United Kingdom

SPTM-P11.8: EXPLOITING AN INTERPLAY BETWEEN NORMS TO ANALYZE SCALAR 4224 QUANTIZATION SCHEMES

Paprimal Parag, Jean-Francois Chamberland, Texas A\&M University, United States

SPTM-P11.9: QUANTIZATION WITH AN ADJUSTABLE CODEWORD LENGTH PENALTY . 4228

W. Bastiaan Kleijn, Victoria University of Wellington, New Zealand; Moo Young Kim, Sejong University, Republic of Korea

SPTM-P11.10: DISCRETE REGRESSION METHODS ON THE CONE OF 4232 POSITIVE-DEFINITE MATRICES

Nicolas Boumal, P.-A. Absil, Université catholique de Louvain, Belgium 


\section{SPTM-P12: ESTIMATION METHODS}

SPTM-P12.1: THE TRANSFORMED VARIATIONAL BAYES APPROXIMATION 4236

Viet Hung Tran, Anthony Quinn, Trinity College Dublin, Ireland

SPTM-P12.2: CROSS-SPECTRUM AND COHERENCE FUNCTION ESTIMATION USING 4240 TIME-DELAYED THOMSON MULTITAPERS

Maria Sandsten, Lund University, Sweden

SPTM-P12.3: IMPROVED ESTIMATION OF THE AMPLITUDE ENVELOPE OF 4244 TIME-DOMAIN SIGNALS USING TRUE ENVELOPE CEPSTRAL SMOOTHING

Marcelo Caetano, Xavier Rodet, Institut de Recherche et Coordination Acoustique/Musique, France

SPTM-P12.4: AN ANALYTIC APPROACH IN JOINT DELAY AND DOPPLER ESTIMATION 4248 USING COPULA

Mohammad Hossein Gholizadeh, Hamidreza Amindavar, Amirkabir University of Technology, Iran

SPTM-P12.5: FAST ALGORITHMS FOR ITERATIVE ADAPTIVE APPROACH SPECTRAL 4252 ESTIMATION TECHNIQUES

George-Othon Glentis, University of Peloponnese, Greece; Andreas Jakobsson, Lund University, Sweden

SPTM-P12.6: AMBIGUITY FUNCTIONS OF COMPRESSIVELY SENSED AND 4256 PROCESSED RADAR WAVEFORMS

Ioannis Kyriakides, University of Nicosia, Cyprus

SPTM-P12.7: MAXIMUM LIKELIHOOD ICA OF QUATERNION GAUSSIAN VECTORS

Javier Via, University of Cantabria, Spain; Daniel P. Palomar, The Hong Kong University of Science and Technology, Hong Kong SAR of China; Luis Vielva, Ignacio Santamaría, University of Cantabria, Spain

SPTM-P12.8: GLOBAL CONVERGENCE OF INDEPENDENT COMPONENT ANALYSIS 4264 BASED ON SEMIDEFINITE PROGRAMMING RELAXATION

Shotaro Akaho, Jun Fujiki, The National Institute of Advanced Industrial Science and Technology, Japan

SPTM-P12.9: ADAPTIVE FREQUENCY-DOMAIN BIASED ESTIMATION ALGORITHMS 4268 WITH AUTOMATIC ADJUSTMENT OF SHRINKAGE FACTORS

Sheng Li, Rodrigo C. de Lamare, University of York, United Kingdom; Martin Haardt, Ilmenau University of Technology, Germany

SPTM-P12.10: A SINGLE SNAPSHOT OPTIMAL FILTERING METHOD FOR 4272 FUNDAMENTAL FREQUENCY ESTIMATION

Jesper Rindom Jensen, Mads Grasbøll Christensen, Sфren Holdt Jensen, Aalborg University, Denmark

SPTM-P12.11: THE BAYESIAN INFERENCE OF PHASE 4276

Anthony Quinn, Trinity College Dublin, Ireland; Jean-Pierre Barbot, Pascal Larzabal, ENS Cachan, France

SPTM-P13: ADAPTIVE FILTERING

SPTM-P13.1: EFFICIENT NLMS AND RLS ALGORITHMS FOR A CLASS OF NONLINEAR 4280 FILTERS USING PERIODIC INPUT SEQUENCES

Alberto Carini, University of Urbino, Italy; V. John Mathews, University of Utah, United States; Giovanni L. Sicuranza, University of Trieste, Italy

SPTM-P13.2: HIGH-ORDER CENTER-FREQUENCY ADAPTIVE FILTERS USING 4284 BLOCK-DIAGRAM-BASED FREQUENCY TRANSFORMATION

Shunsuke Koshita, Yuki Kumamoto, Masahide Abe, Masayuki Kawamata, Tohoku University, Japan

SPTM-P13.3: ON THE INSTANTANEOUS FREQUENCY SMOOTHING FOR SIGNALS 4288 WITH QUASI-LINEAR FREQUENCY CHANGES

Maciej Niedzwiecki, Michal Meller, Gdansk University of Technology, Poland 
TO ONLINE SPARSE SIGNAL RECOVERY

Konstantinos Slavakis, University of Peloponnese, Greece; Yannis Kopsinis, Sergios Theodoridis, University of Athens, Greece

SPTM-P13.5: ACCELERATION OF ADAPTIVE PROXIMAL FORWARD-BACKWARD 4296

SPLITTING METHOD AND ITS APPLICATION TO SPARSE SYSTEM IDENTIFICATION

Masao Yamagishi, Tokyo Institute of Technology, Japan; Masahiro Yukawa, Niigata University, Japan; Isao Yamada, Tokyo

Institute of Technology, Japan

SPTM-P13.6: A NOVEL TRACKING ANALYSIS OF THE NORMALIZED LEAST MEAN 4300 FOURTH ALGORITHM

Muhammad Moinuddin, Iqra University, Pakistan; Azzedine Zerguine, King Fahd University of Petroelum \& Minerals, Saudi Arabia

SPTM-P13.7: IAA SPECTRAL ESTIMATION: FAST IMPLEMENTATION USING THE 4304 GOHBERG-SEMENCUL FACTORIZATION

Ming Xue, Luzhou Xu, Jian Li, University of Florida, United States; Petre Stoica, Uppsala University, Sweden

SPTM-P13.8: GENERALIZED LOW-RANK DECOMPOSITIONS WITH SWITCHING AND 4308 ADAPTIVE ALGORITHMS FOR SPACE-TIME ADAPTIVE PROCESSING

Rodrigo C. de Lamare, University of York, United Kingdom

SPTM-P13.9: ADAPTIVE RECURSIVE FLANN FILTERS FOR NONLINEAR ACTIVE NOISE 4312 CONTROL

Giovanni L. Sicuranza, University of Trieste, Italy; Alberto Carini, University of Urbino, Italy

\section{SPTM-P14: SIGNAL PROCESSING APPLICATIONS}

SPTM-P14.1: NONLINEAR PROPERTIES OF SNORING SOUNDS. 4316

Ali Azarbarzin, Zahra Moussavi, University of Manitoba, Canada

SPTM-P14.2: HOW TO PERFORM TEXTURE RECOGNITION FROM STOCHASTIC

MODELING IN THE WAVELET DOMAIN

Abdourrahmane Atto, Yannick Berthoumieu, University of Bordeaux, France

SPTM-P14.3: CORRELATION MATRIX INTERPOLATION IN SOUND SOURCE LOCALIZATION FOR A ROBOT

Keisuke Nakamura, Kazuhiro Nakadai, Hirofumi Nakajima, Gokhan Ince, Honda Research Institute Japan Co. Ltd., Japan

SPTM-P14.4: DETECTING OSCILLATING SINGULARITIES IN MULTIFRACTAL ANALYSIS : 4328 APPLICATION TO HYDRODYNAMIC TURBULENCE

Patrice Abry, Stephane Roux, Ecole Normale Superieure de Lyon, France; Stephane Jaffard, Universite Paris XII, France

SPTM-P14.5: SINGLE TARGET TRACKING USING VECTOR MAGNETOMETERS.

Niklas Wahlström, Jonas Callmer, Fredrik Gustafsson, Linköping University, Sweden

SPTM-P14.6: DETECTING LOW-RATE PERIODIC EVENTS IN INTERNET TRAFFIC 4336 USING RENEWAL THEORY

Sean McPherson, Antonio Ortega, University of Southern California, United States

SPTM-P14.7: ROBUST ADAPTIVE EVENT DETECTION IN NON-INTRUSIVE LOAD 4340 MONITORING FOR ENERGY AWARE SMART FACILITIES

Yuanwei Jin, Eniye Tebekaemi, University of Maryland Eastern Shore, United States; Mario Berges, Lucio Soibelman, Carnegie Mellon University, United States

SPTM-P14.8: ROBUST GNSS SIGNAL DETECTION IN THE PRESENCE OF NAVIGATION 4344 DATA BITS

Chandrasekhar J, Chandra R. Murthy, Indian Institute of Science, India 
SYSTEMS

Frédéric Faurie, Audrey Giremus, Université Bordeaux 1, France

SPTM-P14.10: A COMBINED LINEAR PROGRAMMING-MAXIMUM LIKELIHOOD 4352 APPROACH TO RADIAL VELOCITY DATA ANALYSIS FOR EXTRASOLAR PLANET DETECTION

Prabhu Babu, Petre Stoica, Uppsala University, Sweden

\section{SPTM-P15: FILTER DESIGN AND FILTER BANKS}

SPTM-P15.1: ON THE CONSTRUCTION OF LOW-PASS FILTERS ON THE UNIT SPHERE 4356

Zubair Khalid, Salman Durrani, Rodney A. Kennedy, Parastoo Sadeghi, Australian Natinoal University, Australia

SPTM-P15.2: ZERO-FORCING AND MMSE FILTERS DESIGN ON THE 2-SPHERE 4360

Liying Wei, Rodney A. Kennedy, Australian National University, Australia

SPTM-P15.3: ADJUSTABLE BANDWIDTH FILTER DESIGN WITH GENERALIZED FARROW STRUCTURE

Chenchi Luo, James McClellan, Georgia Institute of Technology, United States

SPTM-P15.4: REDUCED-HARDWARE DIGITAL FILTER DESIGN VIA JOINT 4368 QUANTIZATION AND MULTIPLE CONSTANT MULTIPLICATION OPTIMIZATION

Matthew Gately, Mark Yeary, Choon Tang, University of Oklahoma, United States

SPTM-P15.5: THE ROLE OF GTD IN OPTIMIZING BIORTHOGONAL FILTER BANKS 4372

Ching-Chih Weng, Palghat P. Vaidyanathan, California Institute of Technology, United States

SPTM-P15.6: SYNTHESIS FILTER BANK OPTIMIZATION WITH LATTICE STRUCTURE 4376 CONSTRAINTS IN 2D SEPARABLE IMAGE PROCESSING

Li Chai, Yuxia Sheng, Wuhan University of Science and Technology, China; Jingxin Zhang, Monash University, Australia

SPTM-P15.7: BOUND-RATIO MINIMIZATION OF FILTER BANK FRAMES BY PERIODIC 4380 PRECODING

Li Chai, Wuhan University of Science and Technology, China; Jingxin Zhang, Monash University, Australia

SPTM-P15.8: DESIGN OF HILBERT TRANSFORM PAIRS OF ORTHONORMAL WAVELET 4384 BASES WITH IMPROVED ANALYTICITY

Xi Zhang, The University of Electro-Communications, Japan

SPTM-P15.9: ON THE DESIGN OF MATCHED ORTHONORMAL WAVELETS WITH 4388 COMPACT SUPPORT

Mohamed Mansour, Texas Instruments Inc., United States

\section{SP-L1: LANGUAGE IDENTIFICATION}

SP-L1.1: NAP FOR HIGH LEVEL LANGUAGE IDENTIFICATION 4392

Fred Richardson, William Campbell, MIT Lincoln Laboratory, United States

SP-L1.2: INFORMATIVE DIALECT RECOGNITION USING CONTEXT-DEPENDENT 4396 PRONUNCIATION MODELING

Nancy Chen, Massachusetts Institute of Technology, United States; Wade Shen, Joseph Campbell, Pedro Torres-Carrasquillo, MIT Lincoln Laboratory, United States

SP-L1.3: LANGUAGE IDENTIFICATION USING A COMBINED ARTICULATORY PROSODY 4400 FRAMEWORK

Abhijeet Sangwan, Mahnoosh Mehrabani, John Hansen, The University of Texas at Dallas, United States 
WITH LARGE PERFORMANCE VARIATION

Raymond W. M. Ng, The Chinese University of Hong Kong, Hong Kong SAR of China; Cheung-Chi Leung, Institute for Infocomm Research, Singapore; Tan Lee, The Chinese University of Hong Kong, Hong Kong SAR of China; Bin Ma, Haizhou Li, Institute

for Infocomm Research, Singapore

SP-L1.5: LANGUAGE IDENTIFICATION FOR SINGING 4408

Mahnoosh Mehrabani, John Hansen, Center for Robust Speech Systems, United States

SP-L1.6: A DYNAMIC APPROACH TO THE SELECTION OF HIGH ORDER N-GRAMS IN 4412 PHONOTACTIC LANGUAGE RECOGNITION

Mikel Penagarikano, Amparo Varona, Luis Javier Rodriguez-Fuentes, German Bordel, University of the Basque Country, Spain

\section{SP-L2: SPEAKER DIARIZATION}

SP-L2.1: SPEAKER DIARIZATION OF MEETINGS BASED ON SPEAKER ROLE N-GRAM 4416 MODELS

Fabio Valente, Deepu Vijayasenan, Petr Motlicek, Idiap Research Institute, Switzerland

SP-L2.2: MULTISTREAM SPEAKER DIARIZATION THROUGH INFORMATION 4420 BOTTLENECK SYSTEM OUTPUTS COMBINATION

Deepu Vijayasenan, Fabio Valente, Petr Motlicek, Idiap Research Institute, Switzerland

SP-L2.3: LINGUISTIC INFLUENCES ON BOTTOM-UP AND TOP-DOWN CLUSTERING 4424 FOR SPEAKER DIARIZATION

Simon Bozonnet, Dong Wang, Nicholas Evans, Raphä̈l Troncy, EURECOM, France

SP-L2.4: FAST SPEAKER DIARIZATION BASED ON BINARY KEYS 4428

Xavier Anguera, Telefonica I+D, Spain; Jean-François Bonastre, University of Avignon, France

SP-L2.5: SPEAKER DIARIZATION OF HETEROGENEOUS WEB VIDEO FILES: A 4432 PRELIMINARY STUDY

Pierre Clement, Université d'Avignon, France; Thierry Bazillon, Aix Marseille Université, France; Corinne Fredouille,

Université d'Avignon, France

SP-L2.6: UNSUPERVISED ACOUSTIC SUB-WORD UNIT DETECTION FOR 4436 QUERY-BY-EXAMPLE SPOKEN TERM DETECTION

Marijn Huijbregts, Mitchell McLaren, David van Leeuwen, Radboud University Nijmegen, Netherlands

\section{SP-L3: ADAPTATION FOR ASR}

SP-L3.1: FRONT-END FEATURE TRANSFORMS WITH CONTEXT FILTERING FOR 4440 SPEAKER ADAPTATION

Jing Huang, IBM T.J. Watson Research Center, United States; Karthik Visweswariah, IBM India Research, India; Peder Olsen, Vaibhava Goel, IBM T.J. Watson Research Center, United States

SP-L3.2: DEFINING THE CONTROLLING PARAMETER IN CONSTRAINED 4444 DISCRIMINATIVE LINEAR TRANSFORM FOR SUPERVISED SPEAKER ADAPTATION

Danning Jiang, IBM China Research Lab, China; Dimitri Kanevsky, Emmanuel Yashchin, IBM T.J. Watson Research Center, United States; Yong Qin, IBM China Research Lab, China

SP-L3.3: SUBSPACE CONSTRAINED LU DECOMPOSITION OF FMLLR FOR RAPID 4448 ADAPTATION

Lei Jia, Dong Yu, Bo Xu, Institute of Automation / Chinese Academy of Sciences, China

SP-L3.4: RAPID FEATURE SPACE MLLR SPEAKER ADAPTATION WITH BILINEAR MODELS 4452 Shilei Zhang, IBM Research Lab - China, China; Peder Olsen, IBM T.J. Watson Research Center, United States; Yong Qin, IBM Research Lab - China, China 
NON-NEGATIVE MATRIX FACTORIZATION

Xueru Zhang, Kris Demuynck, Hugo Van hamme, Katholieke Universiteit Leuven, Belgium

SP-L3.6: A BASIS METHOD FOR ROBUST ESTIMATION OF CONSTRAINED MLLR

Daniel Povey, Kaisheng Yao, Microsoft Corporation, United States

\section{SP-L4: SPEECH ANALYSIS I}

SP-L4.1: NOISE-ROBUST FO ESTIMATION USING SNR-WEIGHTED SUMMARY 4464 CORRELOGRAMS FROM MULTI-BAND COMB FILTERS

Lee Ngee Tan, Abeer Alwan, University of California Los Angeles, United States

SP-L4.2: MPTRACKER: A NEW MULTI-PITCH DETECTION AND SEPARATION 4468 ALGORITHM FOR MIXED SPEECH SIGNALS

Hossein Radfar, University of Toronto, Canada; R. M. Dansereau, Carleton University, Canada; Wai-Yip Chan, Queen's University Belfast, Canada; W. Wong, University of Toronto, Canada

SP-L4.3: UT-SCOPE: TOWARDS LVCSR UNDER LOMBARD EFFECT INDUCED BY 4472 VARYING TYPES AND LEVELS OF NOISY BACKGROUND

Hynek Boril, John H.L. Hansen, The University of Texas at Dallas, United States

SP-L4.4: DISCRIMINATIVE DURATION MODELING FOR SPEECH RECOGNITION WITH 4476 SEGMENTAL CONDITIONAL RANDOM FIELDS

Justine Kao, Stanford University, United States; Geoffrey Zweig, Patrick Nguyen, Microsoft Research, United States

SP-L4.5: QUANTIFYING PERTURBATIONS IN TEMPORAL DYNAMICS FOR AUTOMATED 4480 ASSESSMENT OF SPASTIC DYSARTHRIC SPEECH INTELLIGIBILITY

Tiago Falk, INRS-EMT, Canada; Richard Hummel, Wai-Yip Chan, Queen's University Belfast, Canada

SP-L4.6: IMPROVING TEXT-INDEPENDENT PHONETIC SEGMENTATION BASED ON 4484 THE MICROCANONICAL MULTISCALE FORMALISM

Vahid Khanagha, Khalid Daoudi, Oriol Pont, Hussein Yahia, INRIA Bordeaux Sud-Ouest, France

\section{SP-L5: ACOUSTIC MODELING I}

SP-L5.1: AN ALTERNATIVE FRONT-END FOR THE AT\&T WATSON LV-CSR SYSTEM 4488

Dimitrios Dimitriadis, Enrico Bocchieri, Diamantino Caseiro, AT\&T Labs Research, United States

SP-L5.2: EXEMPLAR-BASED SPARSE REPRESENTATION PHONE IDENTIFICATION 4492 FEATURES

Tara Sainath, David Nahamoo, Bhuvana Ramabhadran, Dimitri Kanevsky, Vaibhava Goel, IBM T.J. Watson Research Center, United States; Parikshit Shah, Massachusetts Institute of Technology, United States

SP-L5.3: CLUSTERING OF BOOTSTRAPPED ACOUSTIC MODEL WITH FULL 4496 COVARIANCE

Xin Chen, University of Missouri, United States; Xiaodong Cui, Jian Xue, Peder Olsen, IBM, United States; John Hersey, Mitsubishi, United States; Bowen Zhou, IBM, United States; Yunxin Zhao, University of Missouri, United States

SP-L5.4: SUBSPACE PURSUIT METHOD FOR KERNEL-LOG-LINEAR MODELS 4500

Yotaro Kubo, Waseda University, Japan; Simon Wiesler, Ralf Schlüter, Hermann Ney, RWTH Aachen University, Germany, Shinji Watanabe, Atsushi Nakamura, NTT Communication Science Laboratories, Japan; Tetsunori Kobayashi, Waseda University, Japan

SP-L5.5: A SYMMETRIZATION OF THE SUBSPACE GAUSSIAN MIXTURE MODEL

Daniel Povey, Microsoft Corporation, United States; Martin Karafiát, Brno University of Technology, Czech Republic; Arnab Ghoshal, University of Saarland, Czech Republic; Petr Schwarz, Brno University of Technology, Czech Republic 
VARIABILITY IN AUTOMATIC SPEECH RECOGNITION

Richard Rose, Shou-Chun Yin, Yun Tang, McGill University, Canada

\section{SP-L6: MISCELLANEOUS SPEAKER IDENTIFICATION}

SP-L6.1: TOWARDS REDUCED FALSE-ALARMS USING COHORTS

Zahi Karam, Massachusetts Institute of Technology / MIT Lincoln Laboratory, United States; William Campbell, MIT Lincoln

Laboratory, United States; Najim Dehak, Massachusetts Institute of Technology, United States

SP-L6.2: SIMPLIFICATION AND OPTIMIZATION OF I-VECTOR EXTRACTION 4516

Ondrej Glembek, Lukas Burget, Pavel Matejka, Martin Karafiát, Brno University of Technology, Czech Republic; Patrick Kenny,

CRIM, Canada

SP-L6.3: SPEAKER CHARACTERIZATION USING SPECTRAL SUBBAND ENERGY RATIO 4520 BASED ON HARMONIC PLUS NOISE MODEL

Yanhua Long, University of Science and Technology of China, China; Zhi-Jie Yan, Frank K. Soong, Microsoft Research Asia, China; Li-Rong Dai, Wu Guo, University of Science and Technology of China, China

SP-L6.4: GIBBS SAMPLING BASED MULTI-SCALE MIXTURE MODEL FOR SPEAKER 4524 CLUSTERING

Shinji Watanabe, Daichi Mochihashi, Takaaki Hori, Atsushi Nakamura, NTT Corporation, Japan

SP-L6.5: AN UTTERANCE COMPARISON MODEL FOR SPEAKER CLUSTERING USING 4528 FACTOR ANALYSIS

Woojay Jeon, Changxue Ma, Dusan Macho, Motorola, United States

SP-L6.6: INTRA-SESSION VARIABILITY COMPENSATION AND A HYPOTHESIS 4532 GENERATION AND SELECTION STRATEGY FOR SPEAKER SEGMENTATION

Carlos Vaquero, Alfonso Ortega, Eduardo Lleida, University of Zaragoza, Spain

\section{SP-L7: SPEAKER VERIFICATION II}

SP-L7.1: A CHANNEL-BLIND SYSTEM FOR SPEAKER VERIFICATION. 4536

Najim Dehak, Zahi Karam, Massachusetts Institute of Technology, United States; Douglas Reynolds, MIT Lincoln Laboratory, United States; Reda Dehak, LRDE-EPITA, France; William Campbell, MIT Lincoln Laboratory, United States; James Glass, Massachusetts Institute of Technology, United States

SP-L7.2: LOG SPECTRA ENHANCEMENT USING SPEAKER DEPENDENT PRIORS FOR 4540 SPEAKER VERIFICATION

Ciira wa Maina, John MacLaren Walsh, Drexel University, United States

SP-L7.3: CLASSIFIER SUBSET SELECTION AND FUSION FOR SPEAKER VERIFICATION. 4544

Filip Sedlak, Tomi Kinnunen, University of Eastern Finland, Finland; Ville Hautamäki, Kong Aik Lee, Haizhou Li, Institute for Infocomm Research, Singapore

SP-L7.4: SPEAKER VERIFICATION USING SPARSE REPRESENTATION CLASSIFICATION 4548 Jia Min Karen Kua, Eliathamby Ambikairajah, Julien Epps, The University of New South Wales, Australia; Roberto Togneri, The University of Western Australia, Australia

SP-L7.5: USE OF VTL-WISE MODELS IN FEATURE-MAPPING FRAMEWORK TO 4552 ACHIEVE PERFORMANCE OF MULTIPLE-BACKGROUND MODELS IN SPEAKER VERIFICATION

Achintya Kumar Sarkar, S. Umesh, Indian Institute of Technology Madras, India 
Marcel Kockmann, Brno University of Technology, Czech Republic; Luciana Ferrer, SRI International, United States; Lukas Burget, Brno University of Technology, Czech Republic; Elizabeth Shriberg, SRI International, United States; Jan Cernocky, Brno University of Technology, Czech Republic

\section{SP-L8: SPEECH SYNTHESIS III}

SP-L8.1: DECISION TREE-BASED CONTEXT CLUSTERING BASED ON CROSS 4560 VALIDATION AND HIERARCHICAL PRIORS

Heiga Zen, Mark Gales, Toshiba Research Europe Ltd., United Kingdom

SP-L8.2: UTILIZING GLOTTAL SOURCE PULSE LIBRARY FOR GENERATING 4564 IMPROVED EXCITATION SIGNAL FOR HMM-BASED SPEECH SYNTHESIS

Tuomo Raitio, Aalto University, Finland; Antti Suni, University of Helsinki, Finland; Hannu Pulakka, Aalto University, Finland; Martti Vainio, University of Helsinki, Finland; Paavo Alku, Aalto University, Finland

SP-L8.3: IMPROVED FO MODELING AND GENERATION IN VOICE CONVERSION 4568

Aki Kunikoshi, The University of Tokyo, Japan; Qian Yao, Frank K. Soong, Microsoft Research Asia, China; Nobuaki Minematsu, The University of Tokyo, Japan

SP-L8.4: JOINT MODELLING OF VOICING LABEL AND CONTINUOUS FO FOR HMM 4572 BASED SPEECH SYNTHESIS

Kai Yu, Steve Young, University of Cambridge, United Kingdom

SP-L8.5: HIGH ACCURATE MODEL-INTEGRATION-BASED VOICE CONVERSION USING 4576 DYNAMIC FEATURES AND MODEL STRUCTURE OPTIMIZATION

Daisuke Saito, The University of Tokyo, Japan; Shinji Watanabe, Atsushi Nakamura, NTT Corporation, Japan; Nobuaki

Minematsu, The University of Tokyo, Japan

SP-L8.6: SYNTHESIZING VISUAL SPEECH TRAJECTORY WITH MINIMUM 4580 GENERATION ERROR

Lijuan Wang, Microsoft Research Asia, China; Yi-Jian Wu, Microsoft Corporation, China; Xiaodan Zhuang, Beckman Institute I University of Illinois at Urbana-Champaign, China; Frank K. Soong, Microsoft Research Asia, China

\section{SP-L9: ROBUST ASR II}

SP-L9.1: SPEAKER AND NOISE FACTORISATION ON THE AURORA4 TASK. 4584

Yongqiang Wang, Mark Gales, University of Cambridge, United Kingdom

SP-L9.2: NON-NEGATIVE MATRIX DECONVOLUTION IN NOISE ROBUST SPEECH 4588 RECOGNITION

Antti Hurmalainen, Tampere University of Technology, Finland; Jort Gemmeke, Radboud University, Netherlands; Tuomas Virtanen, Tampere University of Technology, Finland

SP-L9.3: ROBUST SPEECH RECOGNITION USING DYNAMIC NOISE ADAPTATION 4592

Steven Rennie, Pierre Dognin, Petr Fousek, IBM, United States

SP-L9.4: COMPARING MULTILAYER PERCEPTRON TO DEEP BELIEF NETWORK 4596 TANDEM FEATURES FOR ROBUST ASR

Oriol Vinyals, Suman Ravuri, University of California Berkeley, United States

SP-L9.5: MODEL-BASED COMPRESSIVE SENSING FOR MULTI-PARTY DISTANT SPEECH RECOGNITION

Afsaneh Asaei, Hervé Bourlard, Volkan Cevher, Idiap Research Institute / Ecole Polytechnique Federale de Lausanne, Switzerland 
Kshitiz Kumar, Rita Singh, Carnegie Mellon University, United States; Bhiksha Raj, Disney Research, United States; Richard Stern, Carnegie Mellon University, United States

\section{SP-L10: MODELING AND ANALYSIS OF SPEECH PRODUCTION}

SP-L10.1: FUNCTION OF PHASE-DISTORTION FOR GLOTTAL MODEL ESTIMATION

4608

Gilles Degottex, Institut de Recherche et Coordination Acoustique/Musique / CNRS, France; Axel Röbel, Xavier Rodet, Institut de Recherche et Coordination Acoustique/Musique, France

SP-L10.2: PHASE-BASED INFORMATION FOR VOICE PATHOLOGY DETECTION 4612

Thomas Drugman, Thomas Dubuisson, Thierry Dutoit, University of Mons, Belgium

SP-L10.3: AUTOMATIC ESTIMATION OF THE SECOND SUBGLOTTAL RESONANCE 4616 FROM NATURAL SPEECH

Harish Arsikere, University of California Los Angeles, United States; Steven Lulich, Washington University in St. Louis, United States; Abeer Alwan, University of California Los Angeles, United States

SP-L10.4: ACOUSTIC-TO-ARTICULATORY INVERSION USING AN EPISODIC MEMORY. 4620

Sebastien Demange, LORIA / INRIA, France; Slim Ouni, University Nancy 2, France

SP-L10.5: A SUBJECT-INDEPENDENT ACOUSTIC-TO-ARTICULATORY INVERSION 4624

Prasanta Ghosh, Shrikanth S. Narayanan, University of Southern California, United States

SP-L10.6: RESOLVING NON-UNIQUENESS IN THE ACOUSTIC-TO-ARTICULATORY 4628 MAPPING

Ananthakrishnan G, Olov Engwall, KTH - Royal Institute of Technology, Sweden

\section{SP-L11: SPEECH ENHANCEMENT III}

SP-L11.1: AN SVM BASED CLASSIFICATION APPROACH TO SPEECH SEPARATION 4632

Kun Han, DeLiang Wang, The Ohio State University, United States

SP-L11.2: AN APPROACH TO SEQUENTIAL GROUPING IN COCHANNEL SPEECH. 4636

Ke Hu, DeLiang Wang, The Ohio State University, United States

SP-L11.3: AN EVALUATION OF NOISE POWER SPECTRAL DENSITY ESTIMATION 4640 ALGORITHMS IN ADVERSE ACOUSTIC ENVIRONMENTS

Jalal Taghia, Ruhr-Universität Bochum, Germany; Jalil Taghia, Nasser Mohammadiha, KTH - Royal Institute of Technology, Sweden; Jinqiu Sang, University of Southampton, United Kingdom; Vaclav Bouse, Siemens Audiological Engineering Group, Germany; Rainer Martin, Ruhr-Universität Bochum, Germany

SP-L11.4: ANALYSIS-SYNTHESIS BASED SPEECH ENHANCEMENT WITH IMPROVED 4644 SPECTRUM ENVELOPE ESTIMATION BY TRACKING SPEECH DYNAMICS

Ruofei Chen, Cheung-Fat Chan, City University of Hong Kong, Hong Kong SAR of China

SP-L11.5: PHONEME SELECTIVE SPEECH ENHANCEMENT USING THE GENERALIZED 4648 PARAMETRIC SPECTRAL SUBTRACTION ESTIMATOR

Amit Das, University of Colorado Boulder / University of Texas at Dallas, United States; John Hansen, The University of Texas at Dallas, United States

SP-L11.6: MODEL-BASED SPEECH ENHANCEMENT USING SNR DEPENDENT MMSE 4652 ESTIMATION

Thomas Esch, Peter Vary, RWTH Aachen University, Germany 
SP-P1.1: LATTICE-BASED UNSUPERVISED ACOUSTIC MODEL TRAINING .... 4656

Thiago Fraga-Silva, Jean-Luc Gauvain, Lori Lamel, Laboratoire d'Informatique pour la Mécanique et les Sciences de l'Ingénieur (LIMSI-CNRS), France

SP-P1.2: IMPROVED MODELS FOR MANDARIN SPEECH-TO-TEXT TRANSCRIPTION 4660

Lori Lamel, Jean-Luc Gauvain, Viet-Bac Le, Ilya Oparin, Sha Meng, CNRS/LIMSI, France

SP-P1.3: UNSUPERVISED DETERMINATION OF EFFICIENT KOREAN LVCSR UNITS 4664 USING A BAYESIAN DIRICHLET PROCESS MODEL

Sakriani Sakti, Andrew Finch, Ryosuke Isotani, Hisashi Kawai, Satoshi Nakamura, NICT, Japan

SP-P1.4: MULTI-VIEW AND MULTI-OBJECTIVE SEMI-SUPERVISED LEARNING FOR 4668 LARGE VOCABULARY CONTINUOUS SPEECH RECOGNITION

Xiaodong Cui, Jing Huang, IBM T.J. Watson Research Center, United States; Jen-Tzung Chien, National Cheng Kung University, Taiwan

SP-P1.5: THE IBM 2009 GALE ARABIC SPEECH TRANSCRIPTION SYSTEM 4672

Brian Kingsbury, Hagen Soltau, George Saon, Stephen Chu, Hong-Kwang Kuo, Lidia Mangu, IBM, United States; Suman Ravuri, Nelson Morgan, Adam Janin, International Computer Science Institute, United States

SP-P1.6: AUTOMATICALLY FINDING SEMANTICALLY CONSISTENT N-GRAMS TO ADD 4676 NEW WORDS IN LVCSR SYSTEMS

Gwénolé Lecorvé, INSA/IRISA, France; Guillaume Gravier, CNRS/IRISA, France; Pascale Sébillot, INSA/IRISA, France

SP-P1.7: USING MORPHEME AND SYLLABLE BASED SUB-WORDS FOR POLISH LVCSR 4680

M. Ali Basha Shaik, Amr El-Desoky Mousa, Ralf Schlüter, Hermann Ney, RWTH-Aachen University, Germany

SP-P1.8: EXPLOITING SPARSENESS OF BACKING-OFF LANGUAGE MODELS FOR 4684 EFFICIENT LOOK-AHEAD IN LVCSR

David Nolden, Hermann Ney, Ralf Schlüter, RWTH Aachen University, Germany

SP-P1.9: LARGE VOCABULARY CONTINUOUS SPEECH RECOGNITION WITH 4688 CONTEXT-DEPENDENT DBN-HMMS

George Dahl, University of Toronto, Canada; Dong Yu, Li Deng, Alex Acero, Microsoft Research, United States

SP-P1.10: PROGRESS IN EXAMPLE BASED AUTOMATIC SPEECH RECOGNITION 4692

Kris Demuynck, Dino Seppi, Hugo Van hamme, Dirk Van Compernolle, Katholieke Universiteit Leuven, Belgium

\section{SP-P2: SPEECH SYNTHESIS I}

SP-P2.1: ACCURATE PARAMETER GENERATION USING FIXED-POINT ARITHMETIC FOR 4696 EMBEDDED HMM-BASED SPEECH SYNTHESIZERS

Nobuyuki Nishizawa, Tsuneo Kato, KDDI R\&D Laboratories Inc., Japan

SP-P2.2: AN OPTIMIZATION ALGORITHM OF INDEPENDENT MEAN AND VARIANCE 4700 PARAMETER TYING STRUCTURES FOR HMM-BASED SPEECH SYNTHESIS

Shinji Takaki, Keiichiro Oura, Yoshihiko Nankaku, Keiichi Tokuda, Nagoya Institute of Technology, Japan

SP-P2.3: HMM-BASED SPEECH SYNTHESISER USING THE LF-MODEL OF THE 4704 GLOTTAL SOURCE

Joao Cabral, University College Dublin, Ireland; Steve Renals, Junichi Yamagishi, Korin Richmond, University of Edinburgh, United Kingdom

SP-P2.4: TONAL CONTEXT LABELING USING QUANTIZED FO SYMBOLS FOR 4708 IMPROVING TONE CORRECTNESS IN AVERAGE-VOICE-BASED SPEECH SYNTHESIS

Vataya Chunwijitra, Takashi Nose, Takao Kobayashi, Tokyo Institute of Technology, Japan 

HMM-BASED TTS: DO WE NEED VOICED/UNVOICED CLASSIFICATION?

Javier Latorre, Mark J. F. Gales, Sabine Buchhol, Kate Knill, Toshiba Research Europe, United Kingdom; Masatsune Tamura, Yamato Ohtani, Masami Akamine, Toshiba Corporate Research \& Development Center, Japan

SP-P2.9: HNM-BASED MFCC+FO EXTRACTOR APPLIED TO STATISTICAL SPEECH 4728 SYNTHESIS

Daniel Erro, Iñaki Sainz, Eva Navas, Inma Hernaez, University of the Basque Country, Spain

SP-P2.10: SUPPORT VECTOR REGRESSION FUSION SCHEME IN PHONE DURATION 4732 MODELING

Alexandros Lazaridis, Iosif Mporas, Todor Ganchev, Nikolaos Fakotakis, University of Patras, Greece

\section{SP-P3: SPEECH ENHANCEMENT I}

SP-P3.1: SPECTRAL MAGNITUDE MINIMUM MEAN-SQUARE ERROR BINARY MASKS 4736 FOR DFT BASED SPEECH ENHANCEMENT

Jesper Jensen, Oticon A/S, Denmark; Richard Hendriks, Delft University of Technology, Netherlands

SP-P3.2: ESTIMATION OF THE NOISE CORRELATION MATRIX

Richard Hendriks, Delft University of Technology, Netherlands; Timo Gerkmann, Ruhr-Universität Bochum, Germany

SP-P3.3: SPECTRAL SUBTRACTION ON REAL AND IMAGINARY MODULATION SPECTRA 4744

Yi Zhang, Yunxin Zhao, University of Missouri Columbia, United States

SP-P3.4: SPEECH ENHANCEMENT WITH MASKING PROPERTIES IN EIGEN-DOMAIN 4748 FOR COLORED NOISE

Chang Huai You, Kong Aik Lee, Cheung-Chi Leung, Institute for Infocomm Research, Singapore

SP-P3.5: A DATA-DRIVEN RESIDUAL GAIN APPROACH FOR TWO-STAGE SPEECH 4752 ENHANCEMENT

Yu Gwang Jin, Chul Min Lee, Kiho Cho, Nam Soo Kim, Seoul National University, Republic of Korea

SP-P3.6: LOG-SPECTRAL AMPLITUDE ESTIMATION WITH GENERALIZED GAMMA 4756 DISTRIBUTIONS FOR SPEECH ENHANCEMENT

Bengt Borgstrom, Abeer Alwan, University of California Los Angeles, United States

SP-P3.7: A NOVEL MULTI-BAND SPECTRAL SUBTRACTION METHOD BASED ON PHASE 4760 MODIFICATION AND MAGNITUDE COMPENSATION

Chao Li, Wen-Ju Liu, Institute of Automation / Chinese Academy of Sciences, China 
MIXTURE MODEL FOR (NON-)STATIONARY NOISE

Balazs Fodor, Tim Fingscheidt, TU Braunschweig, Germany

SP-P3.10: AN OPTIMAL FILTERING FOR UNMASKED NOISE PREVENTION.

4772

Asmaa Amehraye, ESIGETEL, France; Lionel Fillatre, Université de technologie de Troyes, France; Dominique Pastor, Telecom Bretagne, France

\section{SP-P4: ROBUST ASR I}

SP-P4.1: SWITCHING LINEAR DYNAMIC TRANSDUCER FOR STEREO DATA BASED 4776 SPEECH FEATURE MAPPING

Chang Woo Han, Tae Gyoon Kang, Doo Hwa Hong, Nam Soo Kim, Seoul National University, Republic of Korea; Kiwan Eom, Jaewon Lee, Samsung Electronics, Republic of Korea

SP-P4.2: A WAVELET-BASED DATA IMPUTATION APPROACH TO SPECTROGRAM 4780 RECONSTRUCTION FOR ROBUST SPEECH RECOGNITION

Shirin Badiezadegan, Richard Rose, McGill University, Canada

SP-P4.3: DELTA-SPECTRAL CEPSTRAL COEFFICIENTS FOR ROBUST SPEECH 4784 RECOGNITION

Kshitiz Kumar, Chanwoo Kim, Richard Stern, Carnegie Mellon University, United States

SP-P4.4: STRUCTURED DISCRIMINATIVE MODELS FOR NOISE ROBUST 4788 CONTINUOUS SPEECH RECOGNITION

Anton Ragni, Mark John Francis Gales, University of Cambridge, United Kingdom

SP-P4.5: FACTOR ANALYSIS BASED VTS AND JUD NOISE ESTIMATION AND 4792 COMPENSATION

Federico Flego, Mark Gales, University of Cambridge, United Kingdom

SP-P4.6: NON-LINEAR NOISE COMPENSATION FOR ROBUST SPEECH RECOGNITION 4796 USING GAUSS-NEWTON METHOD

Yong Zhao, Biing-Hwang(Fred) Juang, Georgia Institute of Technology, United States

SP-P4.7: ROBUST SPEECH RECOGNITION USING MULTIPLE PRIOR MODELS FOR 4800 SPEECH RECONSTRUCTION

Arun Narayanan, Xiaojia Zhao, DeLiang Wang, Eric Fosler-Lussier, The Ohio State University, United States

SP-P4.8: INVESTIGATIONS INTO THE INCORPORATION OF THE IDEAL BINARY MASK 4804

IN ASR

William Hartmann, Eric Fosler-Lussier, The Ohio State University, United States

SP-P4.9: A PITCH BASED NOISE ESTIMATION TECHNIQUE FOR ROBUST SPEECH

RECOGNITION WITH MISSING DATA

Juan Andres Morales Cordovilla, Universidad de Granada, Spain; Ning Ma, University of Sheffield, United Kingdom; Victoria Sanchez Calle, José Luis Carmona Maqueda, Antonio Miguel Peinado Herreros, Universidad de Granada, Spain; Jon Barker,

University of Sheffield, United Kingdom

SP-P4.10: CROSS-CHANNEL SPECTRAL SUBTRACTION FOR MEETING SPEECH 4812 RECOGNITION

Yu Nasu, Koichi Shinoda, Sadaoki Furui, Tokyo Institute of Technology, Japan

SP-P4.11: NON-STATIONARY NOISE ESTIMATION METHOD BASED ON BIAS-RESIDUAL 4816 COMPONENT DECOMPOSITION FOR ROBUST SPEECH RECOGNITION

Masakiyo Fujimoto, Shinji Watanabe, Tomohiro Nakatani, NTT Communication Science Laboratories, Japan 


\section{SP-P5: SPEAKER VERIFICATION I}

SP-P5.1: A LOGARITHMIC BASED POLE-ZERO VOCAL TRACT MODEL ESTIMATION FOR

SPEAKER VERIFICATION

Ewald Enzinger, Peter Balazs, Acoustics Research Institute, Austria; Damián Marelli, University of Newcastle, Australia; Timo

Becker, Federal Criminal Police Office, Germany

SP-P5.2: WELL-CALIBRATED HEAVY TAILED BAYESIAN SPEAKER VERIFICATION FOR 4824 MICROPHONE SPEECH

Mohammed Senoussaoui, École de Technologie Supérieure / Centre de Recherche Informatique de Montréal, Canada; Patrick Kenny, Centre de Recherche Informatique de Montréal, Canada; Pierre Dumouchel, École de Technologie Supérieure / Centre de Recherche Informatique de Montréal, Canada; Fabio Castaldo, Loquendo Politecnico di Torino, Italy

SP-P5.3: FULL-COVARIANCE UBM AND HEAVY-TAILED PLDA IN I-VECTOR SPEAKER 4828 VERIFICATION

Pavel Matejka, Ondrej Glembek, Brno University of Technology, Czech Republic; Fabio Castaldo, Loquendo, Italy; Md Jahangir Alam, INRS-EMT / CRIM, Canada; Oldrich Plchot, Brno University of Technology, Czech Republic; Patrick Kenny, CRIM, Canada; Lukas Burget, Jan Cernocky, Brno University of Technology, Czech Republic

SP-P5.4: DISCRIMINATIVELY TRAINED PROBABILISTIC LINEAR DISCRIMINANT 4832 ANALYSIS FOR SPEAKER VERIFICATION

Lukas Burget, Oldrich Plchot, Brno University of Technology, Czech Republic; Sandro Cumani, Politecnico di Torino, Italy; Ondrej Glembek, Pavel Matejka, Brno University of Technology, Czech Republic; Niko Brummer, AGNITIO, South Africa

SP-P5.5: FEATURE NORMALIZATION FOR SPEAKER VERIFICATION IN ROOM

REVERBERATION

Sriram Ganapathy, The Johns Hopkins University, United States; Jason Pelecanos, Mohamed Kamal Omar, IBM T.J. Watson

Research Center, United States

SP-P5.6: COMPENSATION OF EXTRINSIC VARIABILITY IN SPEAKER VERIFICATION 4840 SYSTEMS ON SIMULATED SKYPE AND HF CHANNEL DATA

Korbinian Riedhammer, Tobias Bocklet, Elmar Noeth, Lehrstuhl f. Informatik 5 (Mustererkennung), Germany

SP-P5.7: DETECTION OF SYNTHETIC SPEECH FOR THE PROBLEM OF IMPOSTURE

Phillip De Leon, New Mexico State University, United States; Inma Hernaez, Ibon Saratxaga, University of Basque Country, Spain; Michael Pucher, Telecommunications Research Center Vienna, Austria; Junichi Yamagishi, University of Edinburgh, United Kingdom

SP-P5.8: EXPLORING IMPLICIT SCORE NORMALIZATION TECHNIQUES IN SPEAKER 4848 VERIFICATION

Ce Zhang, Rong Zheng, Bo Xu, Institute of Automation / Chinese Academy of Sciences, China

SP-P5.9: FAST DISCRIMINATIVE SPEAKER VERIFICATION IN THE I-VECTOR SPACE

Sandro Cumani, Politecnico di Torino, Italy; Niko Brummer, AGNITIO, South Africa; Lukas Burget, Brno University of Technology, Czech Republic; Pietro Laface, Politecnico di Torino, Italy

SP-P5.10: FACTORED COVARIANCE MODELING FOR TEXT-INDEPENDENT SPEAKER 4856 VERIFICATION

Eryu Wang, University of Science and Technology of China, China; Kong Aik Lee, Bin Ma, Haizhou Li, Institute for Infocomm Research, Singapore; Wu Guo, Li-Rong Dai, University of Science and Technology of China, China

\section{SP-P6: MODELING FOR ASR}

SP-P6.1: A MULTI-STREAM ASR FRAMEWORK FOR BLSTM MODELING OF 4860 CONVERSATIONAL SPEECH

Martin Woellmer, Florian Eyben, Technische Universitaet Muenchen, Germany; Björn Schuller, Technische Universität München, Germany; Gerhard Rigoll, Technische Universitaet Muenchen, Germany 
Serena Soldo, Mathew Magimai.-Doss, Joel Pinto, Hervé Bourlard, Idiap Research Institute, Switzerland

SP-P6.3: PHONEME RECOGNITION USING BOOSTED BINARY FEATURES

Anindya Roy, Mathew Magimai.-Doss, Idiap Research Institute / Ecole Polytechnique Federale de Lausanne, Switzerland;

Sebastien Marcel, Idiap Research Institute, Switzerland

SP-P6.4: INVESTIGATION OF ACOUSTIC UNITS FOR LVCSR SYSTEMS.

4872

Xunying Liu, Mark Gales, University of Cambridge, United Kingdom; Jim Hieronymus, International Computer Science Institute, United Kingdom; Phil Woodland, University of Cambridge, United Kingdom

SP-P6.5: SPEECH-BASED IDENTIFICATION OF SOCIAL GROUPS IN A SINGLE ACCENT 4876 OF BRITISH ENGLISH BY HUMANS AND COMPUTERS

Abualsoud Hanani, Martin Russell, Michael Carey, University of Birmingham, United Kingdom

SP-P6.6: APPLICATION SPECIFIC LOSS MINIMIZATION USING GRADIENT BOOSTING

Bin Zhang, University of Washington, United States; Abhinav Sethy, Tara Sainath, Bhuvana Ramabhadran, IBM T.J. Watson

Research Center, United States

SP-P6.7: COMPUTER-ASSISTED TRANSCRIPTION OF SPEECH BASED ON CONFUSION 4884 NETWORK REORDERING

Antoine Laurent, Sylvain Meignier, Teva Merlin, Paul Deléglise, LIUM (Université du Maine), France

SP-P6.8: SPEECH RECOGNITION MODELING ADVANCES FOR MOBILE VOICE SEARCH. 4888

Enrico Bocchieri, Diamantino Caseiro, Dimitrios Dimitriadis, AT\&T Labs Research, United States

SP-P6.9: EIGENTRIPHONES: A BASIS FOR CONTEXT-DEPENDENT ACOUSTIC 4892 MODELING

Tom Ko, Brian Mak, The Hong Kong University of Science and Technology, Hong Kong SAR of China

SP-P6.10: A SIMPLIFIED SUBSPACE GAUSSIAN MIXTURE TO COMPACT ACOUSTIC 4896 MODELS FOR SPEECH RECOGNITION

Bouallegue Mohamed, Matrouf Driss, Georges Linarès, University of Avignon, France

\section{SP-P7: LEXICAL MODELING}

SP-P7.1: LEXICAL ACCESS EXPERIMENTS WITH CONTEXT-DEPENDENT 4900 ARTICULATORY FEATURE-BASED MODELS

Preethi Jyothi, The Ohio State University, United States; Karen Livescu, Toyota Technological Institute at Chicago, United States; Eric Fosler-Lussier, The Ohio State University, United States

SP-P7.2: LEARNING NON-PARAMETRIC MODELS OF PRONUNCIATION. 4904

Brian Hutchinson, University of Washington, United States; Jasha Droppo, Microsoft Research, United States

SP-P7.3: PRONUNCIATION VARIANTS GENERATION USING SMT-INSPIRED 4908 APPROACHES

Panagiota Karanasou, Lori Lamel, LIMSI-CNRS, France

SP-P7.4: POWERFUL EXTENSIONS TO CRFS FOR GRAPHEME TO PHONEME 4912 CONVERSION

Stefan Hahn, Patrick Lehnen, Hermann Ney, RWTH Aachen University, Germany

SP-P7.5: INCORPORATING ALIGNMENTS INTO CONDITIONAL RANDOM FIELDS FOR 4916 GRAPHEME TO PHONEME CONVERSION

Patrick Lehnen, Stefan Hahn, Andreas Guta, Hermann Ney, RWTH Aachen University, Germany

SP-P7.6: EM-STYLE OPTIMIZATION OF HIDDEN CONDITIONAL RANDOM FIELDS FOR 4920 GRAPHEME-TO-PHONEME CONVERSION

Georg Heigold, Stefan Hahn, Patrick Lehnen, Hermann Ney, RWTH Aachen University, Germany 
Line Adde, Torbjørn Svendsen, Norwegian University of Science and Technology, Norway

SP-P7.9: EXTENDED VITERBI ALGORITHM FOR OPTIMIZED WORD HMMS 4932

Michael Gerber, Tobias Kaufmann, Beat Pfister, ETH Zürich, Switzerland

SP-P7.10: RAPID PHONETIC TRANSCRIPTION USING EVERYDAY LIFE NATURAL CHAT 4936

ALPHABET ORTHOGRAPHY FOR DIALECTAL ARABIC SPEECH RECOGNITION

Mohamed Elmahdy, German University in Cairo, Egypt; Rainer Gruhn, University of Ulm, Germany; Slim Abdennadher,

German University in Cairo, Egypt; Wolfgang Minker, University of Ulm, Germany

\section{SP-P8: SPEECH ANALYSIS II}

SP-P8.1: SENTENCE LEVEL EMOTION RECOGNITION BASED ON DECISIONS FROM 4940 SUBSENTENCE SEGMENTS

Je Hun Jeon, Rui Xia, Yang Liu, The University of Texas at Dallas, United States

SP-P8.2: A STUDY OF THE EFFECT OF EMOTIONAL STATE UPON 4944 TEXT-INDEPENDENT SPEAKER IDENTIFICATION

Marius Vasile Ghiurcau, Corneliu Rusu, Technical University of Cluj-Napoca, Romania; Jaakko Astola, Tampere University of Technology, Finland

SP-P8.3: ON-LINE SPEAKER ADAPTATION BASED EMOTION RECOGNITION USING 4948 INCREMENTAL EMOTIONAL INFORMATION

Jae-Bok Kim, Jeong-Sik Park, Yung-Hwan Oh, Korea Advanced Institute of Science and Technology, Republic of Korea

SP-P8.4: FO RANGE AND PEAK ALIGNMENT ACROSS SPEAKERS AND EMOTIONS

Eric Morley, Jan van Santen, Esther Klabbers, Alexander Kain, Oregon Health and Science University, United States

SP-P8.5: EMOTION CLASSIFICATION FROM SPEECH USING EVALUATOR 4956 RELIABILITY-WEIGHTED COMBINATION OF RANKED LISTS

Kartik Audhkhasi, Shrikanth S. Narayanan, University of Southern California, United States

SP-P8.6: ANALYSIS OF ANGER ACROSS SEVERAL AGENT-CUSTOMER INTERACTIONS IN 4960 FRENCH CALL CENTERS

Clement Chastagnol, Laurence Devillers, University of Orsay PXI, France

SP-P8.7: TALKER-TO-LISTENER DISTANCE EFFECTS ON THE VARIATIONS OF THE 4964 INTENSITY AND THE FUNDAMENTAL FREQUENCY OF SPEECH

Thibaut Fux, French-German Research Institute, France; Gang Feng, GIPSA-Lab, France; Véronique Zimpfer, French-German Research Institute, France

SP-P8.8: SHOUT DETECTION IN NOISE 4968

Jouni Pohjalainen, Paavo Alku, Aalto University, Finland; Tomi Kinnunen, University of Eastern Finland, Finland

SP-P8.9: JOINT ANALYSIS OF FO AND SPEECH RATE WITH FUNCTIONAL DATA ANALYSIS. 4972

Michele Gubian, Lou Boves, Radboud University Nijmegen, Netherlands; Francesco Cangemi, University of Provence, France

SP-P8.10: A BINAURAL ALGORITHM FOR SPACE AND PITCH DETECTION.... 4976

Wen-Sheng Chou, Kah-Meng Cheong, Tai-Shih Chi, National Chiao Tung University, Taiwan 
SP-P9.1: ACCURATE TRANSCRIPTION OF BROADCAST NEWS SPEECH USING 4980 MULTIPLE NOISY TRANSCRIBERS AND UNSUPERVISED RELIABILITY METRICS

Kartik Audhkhasi, Panayiotis Georgiou, Shrikanth S. Narayanan, University of Southern California, United States

SP-P9.2: LEVERAGING THE WEB FOR AUTOMATICALLY GENERATING INDEXABLE AND 4984 BROWSABLE KEYWORDS FOR SPEECH FILES

Kit Thambiratnam, Gang Li, Microsoft Research, China; Sha Meng, Tsinghua University, China; Frank Seide, Microsoft

Research, China

SP-P9.3: USING MULTIPLE VISUAL TANDEM STREAMS IN AUDIO-VISUAL SPEECH 4988 RECOGNITION

Ibrahim Saygin Topkaya, Hakan Erdogan, Sabanci University, Turkey

SP-P9.4: MAKING THE MOST FROM MULTIPLE MICROPHONES IN MEETING 4992 RECOGNITION

Andreas Stolcke, SRI International, United States

SP-P9.5: ACOUSTIC DATA SHARING FOR AFGHAN AND PERSIAN LANGUAGES

4996

Arindam Mandal, Dimitra Vergyri, Murat Akbacak, Colleen Richey, Andreas Kathol, SRI International, United States

SP-P9.6: CROSS-LANGUAGE BOOTSTRAPPING BASED ON COMPLETELY 5000 UNSUPERVISED TRAINING USING MULTILINGUAL A-STABIL

Ngoc Thang Vu, Franziska Kraus, Tanja Schultz, Karlsruhe Institute of Technology, Germany

SP-P9.7: ASYMMETRIC ACOUSTIC MODELING OF MIXED LANGUAGE SPEECH

Ying Li, Pascale Fung, Ping Xu, Yi Liu, The Hong Kong University of Science and Technology, Hong Kong SAR of China

SP-P9.8: USING STACKED TRANSFORMATIONS FOR RECOGNIZING FOREIGN 5008 ACCENTED SPEECH

Peter Smit, Mikko Kurimo, Aalto University, Finland

SP-P9.9: LANGUAGE DEPENDENT UNIVERSAL PHONEME POSTERIOR ESTIMATION 5012 FOR MIXED LANGUAGE SPEECH RECOGNITION

David Imseng, Hervé Bourlard, Mathew Magimai.-Doss, John Dines, Idiap Research Institute, Switzerland

SP-P9.10: RECOGNIZING ENGLISH QUERIES IN MANDARIN VOICE SEARCH 5016

Hung-An Chang, Massachusetts Institute of Technology, United States; Yun-Hsuan Sung, Brian Strope, Francoise Beaufays, Google Inc., United States

SP-P9.11: BILINGUAL ACOUSTIC MODELING WITH STATE MAPPING AND THREE-STAGE 5020 ADAPTATION FOR TRANSCRIBING UNBALANCED CODE-MIXED LECTURES

Ching-Feng Yeh, Liang-Che Sun, Chao-Yu Huang, Lin-Shan Lee, National Taiwan University, Taiwan

\section{SP-P10: STATISTICAL METHODS FOR ASR}

SP-P10.1: MLP BASED PHONEME DETECTORS FOR AUTOMATIC SPEECH 5024 RECOGNITION

Samuel Thomas, The Johns Hopkins University, United States; Patrick Nguyen, Microsoft Research, United States; Geoffrey Zweig, Microsoft Corporation, United States; Hynek Hermansky, The Johns Hopkins University, United States

SP-P10.2: DIRICHLET MIXTURE MODELS OF NEURAL NET POSTERIORS FOR 5028 HMM-BASED SPEECH RECOGNITION

Balakrishnan Varadarajan, Sivaram Garimella, Sanjeev Khudanpur, The Johns Hopkins University, United States 
Ariya Rastrow, Markus Dreyer, The Johns Hopkins University, United States; Abhinav Sethy, IBM, United States; Sanjeev Khudanpur, The Johns Hopkins University, United States; Bhuvana Ramabhadran, IBM, United States; Mark Dredze, The Johns Hopkins University, United States

SP-P10.4: AUTOMATIC SPEECH RECOGNITION USING HIDDEN CONDITIONAL NEURAL 5036 FIELDS

Yasuhisa Fujii, Kazumasa Yamamoto, Seiichi Nakagawa, Toyohashi University of Technology, Japan

SP-P10.5: A HIERARCHICAL, CONTEXT-DEPENDENT NEURAL NETWORK 5040 ARCHITECTURE FOR IMPROVED PHONE RECOGNITION

László Tóth, Hungarian Academy of Sciences, Hungary

SP-P10.6: SPEECH RECOGNITION WITH SEGMENTAL CONDITIONAL RANDOM 5044 FIELDS: A SUMMARY OF THE JHU CLSP 2010 SUMMER WORKSHOP

Geoffrey Zweig, Patrick Nguyen, Microsoft Research, United States; Dirk Van Compernolle, Kris Demuynck, Katholieke Universiteit Leuven, Belgium; Les Atlas, Pascal Clark, University of Washington, United States; Gregory Sell, Stanford University, United States; Meihong Wang, Fei Sha, University of Southern California, United States; Hynek Hermansky, Damianos Karakos, Aren Jansen, Samuel Thomas, Sivaram G.S.V.S., The Johns Hopkins University, United States; Sam Bowman, University of Chicago, United States; Justine Kao, Stanford University, United States

SP-P10.7: INTEGRATING META-INFORMATION INTO EXEMPLAR-BASED SPEECH 5048 RECOGNITION WITH SEGMENTAL CONDITIONAL RANDOM FIELDS

Kris Demuynck, Dino Seppi, Dirk Van Compernolle, Katholieke Universiteit Leuven, Belgium; Patrick Nguyen, Geoffrey Zweig, Microsoft Research, United States

SP-P10.8: ENRICHING MANDARIN SPEECH RECOGNITION BY INCORPORATING A 5052 HIERARCHICAL PROSODY MODEL

Jyh-Her Yang, Ming-Chieh Liu, Hao-Hsiang Chang, Chen-Yu Chiang, Yih-Ru Wang, Sin-Horng Chen, National Chiao Tung University, Taiwan

SP-P10.9: BAYESIAN SENSING HIDDEN MARKOV MODELS FOR SPEECH 5056 RECOGNITION

George Saon, IBM T.J. Watson Research Center, United States; Jen-Tzung Chien, National Cheng Kung University, Taiwan

SP-P10.10: DEEP BELIEF NETWORKS USING DISCRIMINATIVE FEATURES FOR PHONE 5060 RECOGNITION

Abdel-rahman Mohamed, University of Toronto, Canada; Tara Sainath, IBM T.J. Watson Research Center, United States; George Dahl, University of Toronto, Canada; Bhuvana Ramabhadran, IBM T.J. Watson Research Center, United States; Geoffrey Hinton, University of Toronto, Canada; Michael Picheny, IBM T.J. Watson Research Center, United States

\section{SP-P11: SPEECH ENHANCEMENT II}

SP-P11.1: SPEECH ENHANCEMENT BASED ON LOG SPECTRAL ENVELOPE MODEL 5064 AND HARMONICITY-DERIVED SPECTRAL MASK, AND ITS COUPLING WITH FEATURE COMPENSATION

Takuya Yoshioka, Tomohiro Nakatani, NTT Corporation, Japan

SP-P11.2: LINEAR PREDICTIVE PERCEPTUAL FILTERING FOR ACOUSTIC VECTOR 5068 SENSORS: EXPLOITING DIRECTIONAL RECORDINGS FOR HIGH QUALITY SPEECH ENHANCEMENT

Muawiyath Shujau, Cristian H. Ritz, University of Wollongong, Australia; Ian S. Burnett, Royal Melbourne Institiute of Technology, Australia 
FAMILY VIA HIGHER-ORDER STATISTICS

Takayuki Inoue, Hiroshi Saruwatari, Kiyohiro Shikano, Nara Institute of Science and Technology, Japan; Kazunobu Kondo,

YAMAHA Corporate, Japan

SP-P11.5: ROBUST BAYESIAN ANALYSIS APPLIED TO WIENER FILTERING OF SPEECH

5080

P. Spencer Whitehead, David V. Anderson, Georgia Institute of Technology, United States

SP-P11.6: CLUSTERING AND SUPPRESSION OF TRANSIENT NOISE IN SPEECH SIGNALS USING DIFFUSION MAPS

Ronen Talmon, Israel Cohen, Technion / Israel Institute of Technology, Israel; Sharon Gannot, Bar-Ilan University, Israel

SP-P11.7: USING A REMOTE WIRELESS MICROPHONE FOR SPEECH ENHANCEMENT 5088

IN NON-STATIONARY NOISE

Sriram Srinivasan, Philips Research, Netherlands

SP-P11.8: DYNAMIC SIGNAL COMBINING FOR DISTRIBUTED MICROPHONE SYSTEMS 5092

IN CAR ENVIRONMENTS

Timo Matheja, Markus Buck, Achim Eichentopf, Nuance Communications Aachen GmbH, Germany

SP-P11.9: ARTIFICIAL BANDWIDTH EXTENSION OF SPECTRAL ENVELOPE WITH 5096 TEMPORAL CLUSTERING

Can Yagli, Engin Erzin, Koc University, Turkey

SP-P11.10: SPEECH BANDWIDTH EXTENSION USING GAUSSIAN MIXTURE 5100 MODEL-BASED ESTIMATION OF THE HIGHBAND MEL SPECTRUM

Hannu Pulakka, Ulpu Remes, Kalle Palomäki, Mikko Kurimo, Paavo Alku, Aalto University, Finland

SP-P11.11: PHASE-SENSITIVE SPEECH ENHANCEMENT FOR COCHLEAR IMPLANT 5104 PROCESSING

Pourya Jafari, York University, Canada; Hou-Yong Kang, Xiaosong Wang, Qian-Jie Fu, House Ear Institute, United States; Hui Jiang, York University, Canada

\section{SP-P12: SPEECH SYNTHESIS II}

SP-P12.1: AN ANALYSIS OF MACHINE TRANSLATION AND SPEECH SYNTHESIS IN 5108 SPEECH-TO-SPEECH TRANSLATION SYSTEM

Kei Hashimoto, Nagoya Institute of Technology, Japan; Junichi Yamagishi, University of Edinburgh, United Kingdom; William Byrne, University of Cambridge, United Kingdom; Simon King, University of Edinburgh, United Kingdom; Keiichi Tokuda, Nagoya Institute of Technology, Japan

SP-P12.2: EVALUATION OF OBJECTIVE MEASURES FOR INTELLIGIBILITY 5112 PREDICTION OF HMM-BASED SYNTHETIC SPEECH IN NOISE

Cassia Valentini Botinhao, Junichi Yamagishi, Simon King, University of Edinburgh, United Kingdom

SP-P12.3: NON-PARALLEL TRAINING FOR VOICE CONVERSION BASED ON FT-GMM 5116

Ling-Hui Chen, Zhen-Hua Ling, Li-Rong Dai, University of Science and Technology of China, China

SP-P12.4: A FRAME MAPPING BASED HMM APPROACH TO CROSS-LINGUAL VOICE 5120 TRANSFORMATION

Yao Qian, Ji Xu, Frank K. Soong, Microsoft Research Asia Beijing, China

SP-P12.5: ONE SENTENCE VOICE ADAPTATION USING GMM-BASED 5124 FREQUENCY-WARPING AND SHIFT WITH A SUB-BAND BASIS SPECTRUM MODEL

Masatsune Tamura, Masahiro Morita, Takehiko Kagoshima, Masami Akamine, Toshiba Corporation, Japan

SP-P12.6: PITCH TRANSPOSITION AND BREATHINESS MODIFICATION USING A 5128 GLOTTAL SOURCE MODEL AND ITS ADAPTED VOCAL-TRACT FILTER

Gilles Degottex, Institut de Recherche et Coordination Acoustique/Musique / CNRS, France; Axel Röbel, Xavier Rodet, Institut de Recherche et Coordination Acoustique/Musique, France 
Pierre Lanchantin, Xavier Rodet, Institut de Recherche et Coordination Acoustique/Musique, France

SP-P12.8: AN EVALUATION OF ALARYNGEAL SPEECH ENHANCEMENT METHODS BASED 5136 ON VOICE CONVERSION TECHNIQUES

Hironori Doi, Keigo Nakamura, Tomoki Toda, Hiroshi Saruwatari, Kiyohiro Shikano, Nara Institute of Science and Technology, Japan

SP-P12.9: REAL-TIME VOICE CONVERSION BASED ON INSTANTANEOUS HARMONIC 5140 PARAMETERS

Elias Azarov, Alexander Petrovsky, Belarusian State University of Informatics and Radioelectronics, Belarus

SP-P12.10: A MULTI-DIMENSIONAL APPROACH TO PREDICTING SPEECH QUALITY 5144 USING A PHYSIOLOGICALLY MOTIVATED MODEL OF THE COCHLEA

Deep Sen, Wenliang Lu, University of New South Wales, Australia

\section{SP-P13: MISCELLANEOUS ASR}

SP-P13.1: JOINT ENCODING OF THE WAVEFORM AND SPEECH RECOGNITION 5148 FEATURES USING A TRANSFORM CODEC

Xing Fan, The University of Texas at Dallas, United States; Michael Seltzer, Jasha Droppo, Henrique Malvar, Alex Acero, Microsoft Research, United States

SP-P13.2: UNSUPERVISED VOCABULARY DISCOVERY USING NON-NEGATIVE MATRIX 5152 FACTORIZATION WITH GRAPH REGULARIZATION

Meng Sun, Hugo Van hamme, Katholieke Universiteit Leuven, Belgium

SP-P13.3: MACHINE AND ACOUSTICAL CONDITION DEPENDENCY ANALYSES FOR FAST 5156 ACOUSTIC LIKELIHOOD CALCULATION TECHNIQUES

Atsunori Ogawa, Satoshi Takahashi, Atsushi Nakamura, NTT Corporation, Japan

SP-P13.4: FORENSICALLY INSPIRED APPROACHES TO AUTOMATIC SPEAKER 5160 RECOGNITION

Kyu Han, Mohamed Kamal Omar, Jason Pelecanos, Cezar Pendus, Sibel Yaman, Weizhong Zhu, IBM, United States

SP-P13.5: A-FUNCTIONS: A GENERALIZATION OF EXTENDED BAUM-WELCH 5164 TRANSFORMATIONS TO CONVEX OPTIMIZATION

Dimitri Kanevsky, David Nahamoo, Tara Sainath, Bhuvana Ramabhadran, Peder Olsen, IBM, United States

SP-P13.6: STRUCTURED PRECISION MODELLING WITH CHOLESKY BASIS 5168 SUPERPOSITION FOR SPEECH RECOGNITION

Lei Jia, Institute of Automation / Chinese Academy of Sciences, China; Kai Yu, University of Cambridge, United Kingdom; Bo $\mathrm{Xu}$, Institute of Automation / Chinese Academy of Sciences, China

SP-P13.7: GESTURE-BASED DYNAMIC BAYESIAN NETWORK FOR NOISE ROBUST 5172 SPEECH RECOGNITION

Vikramjit Mitra, University of Maryland College Park, United States; Hosung Nam, Haskins Laboratories, United States; Carol Espy-Wilson, University of Maryland College Park, United States; Elliot Saltzman, Boston University, United States; Louis Goldstein, University of Southern California, United States

SP-P13.8: DYNAMIC SELECTION OF A SPEECH ENHANCEMENT METHOD FOR 5176 ROBUST SPEECH RECOGNITION IN MOVING MOTORCYCLE ENVIRONMENT Iosif Mporas, Todor Ganchev, Otilia Kocsis, Nikolaos Fakotakis, University of Patras, Greece 


\section{SP-P14: ACOUSTIC MODELING II}

SP-P14.1: SPEECH INVERSION: BENEFITS OF TRACT VARIABLES OVER PELLET 5188

TRAJECTORIES

Vikramjit Mitra, University of Maryland College Park, United States; Hosung Nam, Haskins Laboratories, United States; Carol Espy-Wilson, University of Maryland College Park, United States; Elliot Saltzman, Boston University, United States; Louis Goldstein, University of Southern California, United States

SP-P14.2: INTEGRATING ARTICULATORY FEATURES USING KULLBACK-LEIBLER 5192 DIVERGENCE BASED ACOUSTIC MODEL FOR PHONEME RECOGNITION

Ramya Rasipuram, Mathew Magimai.-Doss, Idiap Research Institute, Switzerland

SP-P14.3: MULTI-STREAM SPECTRO-TEMPORAL AND CEPSTRAL FEATURES BASED ON 5196 DATA-DRIVEN HIERARCHICAL PHONEME CLUSTERS

Shang-wen Li, Liang-che Sun, Lin-Shan Lee, National Taiwan University, Taiwan

SP-P14.4: ARCCOSINE KERNELS: ACOUSTIC MODELING WITH INFINITE NEURAL 5200 NETWORKS

Chih-Chieh Cheng, University of California San Diego, United States; Brian Kingsbury, IBM T.J. Watson Research Center,

United States

SP-P14.5: NON-STATIONARY FEATURE EXTRACTION FOR AUTOMATIC SPEECH 5204 RECOGNITION

Zoltán Tüske, Pavel Golik, Ralf Schlüter, RWTH Aachen University, Germany; Friedhelm R. Drepper, Forschungszentrum Jülich, Germany

SP-P14.6: TONE AND PITCH ACCENT CLASSIFICATION USING AUDITORY ATTENTION 5208 CUES

Ozlem Kalinli, SONY Computer Entertainment America, United States

SP-P14.7: ON THE USE OF IDEAL BINARY MASKS FOR IMPROVING PHONETIC 5212 CLASSIFICATION

Arun Narayanan, DeLiang Wang, The Ohio State University, United States

SP-P14.8: OVERLAPPED SPEECH DETECTION USING LONG-TERM 5216 SPECTRO-TEMPORAL SIMILARITY IN STEREO RECORDING

Bo Xiao, Prasanta Ghosh, Panayiotis Georgiou, Shrikanth S. Narayanan, University of Southern California, United States

SP-P14.9: A MODIFIED MAP CRITERION BASED ON HIDDEN MARKOV MODEL FOR 5220 VOICE ACTIVITY DETECION

Shiwen Deng, Jiqing Han, Tieran Zheng, Guibin Zheng, Harbin Institute of Technology, China

SP-P14.10: ACOUSTIC MODEL TRAINING FOR NON-AUDIBLE MURMUR RECOGNITION 5224 USING TRANSFORMED NORMAL SPEECH DATA

Denis Babani, Tomoki Toda, Hiroshi Saruwatari, Kiyohiro Shikano, Nara Institute of Science and Technology, Japan

\section{SP-P15: SPEECH CODING AND ANALYSIS}

SP-P15.1: CODING OF UNQUANTIZED SPECTRUM SUB-BANDS IN SUPERWIDEBAND 5228 AUDIO CODECS

Václav Eksler, Milan Jelínek, VoiceAge Corp., Canada

SP-P15.2: G.711.1 ANNEX D AND G.722 ANNEX B - NEW ITU-T SUPERWIDEBAND CODECS 5232

Lei Miao, Zexin Liu, Chen Hu, Huawei Technologies Co. Ltd., China; Václav Eksler, VoiceAge Corp., Canada; Stéphane Ragot, Claude Lamblin, Balazs Kovesi, France Telecom Orange, France; Jongmo Sung, Electronics and Telecommunications Research Institute, Republic of Korea; Masahiro Fukui, Shigeaki Sasaki, Yusuke Hiwasaki, NTT Cyber Space Laboratories, Japan 
MSD-HMM WITH QUANTIZED FO CONTEXT

Takashi Nose, Takao Kobayashi, Tokyo Institute of Technology, Japan

SP-P15.4: BIT RATE REDUCTION OF THE MELP CODER USING LEMPEL-ZIV

SEGMENT QUANTIZATION

Minoru Kohata, Chiba Institute of Technology, Japan; Motoyuki Suzuki, The University of Tokushima, Japan; Akinori Ito,

Tohoku University, Japan; Shozo Makino, Tohoku Bunka Gakuen University, Japan

SP-P15.5: CORRELATION PROPERTIES OF QUANTIZATION NOISE

Peter Kabal, McGill University, Canada

SP-P15.6: RE-ENGINEERING ITU-T G.722: LOW DELAY AND COMPLEXITY

SUPERWIDEBAND CODING AT 64 KBIT/S WITH G.722 BITSTREAM WATERMARKING

Balazs Kovesi, Stéphane Ragot, Claude Lamblin, France Telecom Orange, France; Lei Miao, Zexin Liu, Chen Hu, Huawei

Technologies Co. Ltd., China

SP-P15.7: ACOUSTIC-PHONETIC INFORMATION FROM EXCITATION SOURCE FOR REFINING MANNER HYPOTHESES OF A PHONE RECOGNIZER

Dhananjaya N., Indian Institute of Technology Madras, India; Yegnanarayana B., Suryakanth V. Gangashetty, International

Institute of Information Technology Hyderabad, India

SP-P15.8: SYLLABIFICATION OF CONVERSATIONAL SPEECH USING BIDIRECTIONAL

5256 LONG-SHORT-TERM MEMORY NEURAL NETWORKS

Christian Landsiedel, Technische Universitaet Muenchen, Germany; Jens Edlund, KTH - Royal Institute of Technology, Sweden; Florian Eyben, Technische Universitaet Muenchen, Germany; Daniel Neiberg, KTH - Royal Institute of Technology, Sweden;

Björn Schuller, Technische Universität München, Germany

SP-P15.9: SPARSE NON-NEGATIVE DECOMPOSITION OF SPEECH POWER SPECTRA 5260 FOR FORMANT TRACKING

Jean-Louis Durrieu, Jean-Philippe Thiran, Ecole Polytechnique Fédérale de Lausanne, Switzerland

SP-P15.10: A NOVEL APPROACH USING MODULATION FEATURES FOR 5264 MULTIPHONE-BASED SPEECH RECOGNITION

Pascal Clark, University of Washington, United States; Gregory Sell, Stanford University, United States; Les Atlas, University of Washington, United States

\section{SP-P16: SPEAKER RECOGNITION I}

SP-P16.1: SOFT FRAME MARGIN ESTIMATION OF GAUSSIAN MIXTURE MODELS FOR 5268 SPEAKER RECOGNITION WITH SPARSE TRAINING DATA

Yan Yin, Qi Li, Li Creative Technologies, Inc., United States

SP-P16.2: THE MIT LL 2010 SPEAKER RECOGNITION EVALUATION SYSTEM: SCALABLE 5272 LANGUAGE-INDEPENDENT SPEAKER RECOGNITION

Douglas Sturim, William Campbell, MIT Lincoln Laboratory, United States; Najim Dehak, Massachusetts Institute of Technology, United States; Zahi Karam, Alan McCree, Douglas Reynolds, Fred Richardson, Pedro Torres-Carrasquillo, MIT Lincoln Laboratory, United States; Stephen Shum, Massachusetts Institute of Technology, United States

SP-P16.3: A PARTIAL LEAST SQUARES FRAMEWORK FOR SPEAKER RECOGNITION 5276

Balaji Vasan Srinivasan, Dmitry Zotkin, Ramani Duraiswami, University of Maryland, United States

SP-P16.4: OPEN-SET SPEAKER IDENTIFICATION IN BROADCAST NEWS 5280

Chao Gao, Guruprasad Saikumar, Amit Srivastava, Premkumar Natarajan, Raytheon BBN Technologies, United States RECOGNITION

Jean-François Bonastre, University of Avignon, France; Xavier Anguera Miro, Telefonica Research, Spain; Pierre-Michel Bousquet, Driss Matrouf, University of Avignon, France 


\section{EVALUATION}

Weiwu Jiang, The Chinese University of Hong Kong, Hong Kong SAR of China; Man-Wai Mak, Wei Rao, Hong Kong Polytechnic University, Hong Kong SAR of China; Helen Meng, The Chinese University of Hong Kong, Hong Kong SAR of China

SP-P16.7: THE SRI NIST 2010 SPEAKER RECOGNITION EVALUATION SYSTEM 5292

Nicolas Scheffer, Luciana Ferrer, Martin Graciarena, SRI International, United States; Sachin Kajarekar, Cisco systems, United States; Elizabeth Shriberg, Andreas Stolcke, SRI International, United States

SP-P16.8: LANGUAGE-INDEPENDENT CONSTRAINED CEPSTRAL FEATURES FOR 5296 SPEAKER RECOGNITION

Elizabeth Shriberg, Andreas Stolcke, SRI International, United States

SP-P16.9: PARALLEL TRANSFORMATION NETWORK FEATURES FOR SPEAKER 5300 RECOGNITION

Alberto Abad, INESC-ID Lisboa, Portugal; Jordi Luque, TALP Research Center, Spain; Isabel Trancoso, INESC-ID Lisboa/IST, Portugal

SP-P16.10: EFFECTIVE BACKGROUND DATA SELECTION IN SVM SPEAKER 5304 RECOGNITION FOR UNSEEN TEST ENVIRONMENT: MORE IS NOT ALWAYS BETTER

Jun-Won Suh, Yun Lei, Wooil Kim, John H.L. Hansen, The University of Texas at Dallas, United States

\section{SP-P17: DISCRIMINATIVE TECHNIQUES FOR ASR}

SP-P17.1: A STUDY OF AN IRRELEVANT VARIABILITY NORMALIZATION BASED 5308 DISCRIMINATIVE TRAINING APPROACH FOR LVCSR

Yu Zhang, Shanghai Jiao Tong University, China; Jian Xu, University of Science and Technology of China, China; Zhi-Jie Yan, Qiang Huo, Microsoft Research Asia Beijing, China

SP-P17.2: DISCRIMINATIVE TRAINING FOR FULL COVARIANCE MODELS 5312

Peder Olsen, Vaibhava Goel, Steven Rennie, IBM T.J. Watson Research Center, United States

SP-P17.3: DISCRIMINATIVE TRAINING FOR BAYESIAN SENSING HIDDEN MARKOV 5316 MODELS

George Saon, IBM T.J. Watson Research Center, United States; Jen-Tzung Chien, National Cheng Kung University, Taiwan

SP-P17.4: INCREASING DISCRIMINATIVE CAPABILITY ON MAP-BASED MAPPING 5320 FUNCTION ESTIMATION FOR ACOUSTIC MODEL ADAPTATION

Yu Tsao, Ryosuke Isotani, Hisashi Kawai, Satoshi Nakamura, National Institute of Information and Communications Technology, Japan

SP-P17.5: FEATURE SELECTION FOR LOG-LINEAR ACOUSTIC MODELS 5324

Simon Wiesler, Alexander Richard, Yotaro Kubo, Ralf Schlüter, Hermann Ney, RWTH Aachen University, Germany

SP-P17.6: DISCRIMINATIVE TRAINING FOR DIRECT MINIMIZATION OF DELETION, 5328 INSERTION AND SUBSTITUTION ERRORS

Sunghwan Shin, Georgia Institute of Technology, United States; Ho-Young Jung, Electronics and Telecommunications Research Institute, Republic of Korea; Biing-Hwang(Fred) Juang, Georgia Institute of Technology, United States

SP-P17.7: RECENT DEVELOPMENT OF DISCRIMINATIVE TRAINING USING 5332 NON-UNIFORM CRITERIA FOR CROSS-LEVEL ACOUSTIC MODELING Chao Weng, Biing-Hwang(Fred) Juang, Georgia Institute of Technology, United States

SP-P17.8: MULTILAYER PERCEPTRON WITH SPARSE HIDDEN OUTPUTS FOR 5336 PHONEME RECOGNITION

Sivaram Garimella, Hynek Hermansky, The Johns Hopkins University, United States 
Langzhou Chen, Mark J. F. Gales, K. K. Chin, Toshiba Research Europe Limited / Cambridge Research Laboratory, United Kingdom

\section{SP-P18: SPEECH SYNTHESIS IV}

SP-P18.1: SIGNIFICANCE OF VOWEL EPENTHESIS IN TELUGU TEXT-TO-SPEECH 5348 SYNTHESIS

Vijayaditya Peddinti, Kishore Prahallad, International Institute of Information Technology Hyderabad, India

SP-P18.2: BUILDING HMM BASED UNIT-SELECTION SPEECH SYNTHESIS SYSTEM 5352 USING SYNTHETIC SPEECH NATURALNESS EVALUATION SCORE

Heng Lu, Zhen-Hua Ling, Li-Rong Dai, Ren-Hua Wang, University of Science and Technology of China, China

SP-P18.3: FURTHER ANALYSIS OF LATENT AFFECTIVE MAPPING FOR NATURALLY 5356 EXPRESSIVE SPEECH SYNTHESIS

Jerome Bellegarda, Apple Inc., United States

SP-P18.4: PROSODIC CONTROL OF UNIT-SELECTION SPEECH SYNTHESIS: A 5360 PROBABILISTIC APPROACH

Christophe Veaux, Xavier Rodet, Institut de Recherche et Coordination Acoustique/Musique, France

SP-P18.5: TOWARD TEXT MESSAGE NORMALIZATION: MODELING ABBREVIATION 5364 GENERATION

Deana Pennell, Yang Liu, The University of Texas at Dallas, United States

SP-P18.6: VOCAL ATTRACTIVENESS OF STATISTICAL SPEECH SYNTHESISERS 5368

Sandra Andraszewicz, Junichi Yamagishi, Simon King, University of Edinburgh, United Kingdom

SP-P18.7: SPEAKER SIMILARITY EVALUATION OF FOREIGN-ACCENTED SPEECH 5372 SYNTHESIS USING HMM-BASED SPEAKER ADAPTATION

Mirjam Wester, University of Edinburgh, United Kingdom; Reima Karhila, Aalto University, Finland

SP-P18.8: USING F0 TO CONSTRAIN THE UNIT SELECTION VITERBI NETWORK. 5376

Alistair Conkie, Ann Syrdal, AT\&T Labs Research, United States

SP-P18.9: SPEECH SYNTHESIS USING HMM BASED DIPHONE INVENTORY 5380 ENCODING FOR LOW-RESOURCE DEVICES

Guntram Strecha, Matthias Wolff, Technische Universität Dresden, Germany

SP-P18.10: IMPROVED POS TAGGING FOR TEXT-TO-SPEECH SYNTHESIS 5384

Ming Sun, The Johns Hopkins University, United States; Jerome Bellegarda, Apple Inc., United States

\section{SP-P19: SPEECH ANALYSIS III}

SP-P19.1: FFT-BASED SPECTRO-TEMPORAL ANALYSIS AND SYSTNESIS OF SOUNDS 5388

Chung-Chien Hsu, Ting-Han Lin, Tai-Shih Chi, National Chiao Tung University, Taiwan

SP-P19.2: STUDY OF ROBUSTNESS OF ZERO FREQUENCY RESONATOR METHOD 5392 FOR EXTRACTION OF FUNDAMENTAL FREQUENCY

Yegnanarayana Bayya, IIIT Hyderabad, India; Prasanna S. R. M., IIT Guwahati, India; Guruprasad S., IIIT Hyderabad, India

SP-P19.3: DECOMPOSITION OF SPEECH SIGNALS FOR ANALYSIS OF APERIODIC 5396 COMPONENTS OF EXCITATION

Yegnanarayana Bayya, Dhananjaya N., Anand Joseph Medabalimi, Suryakanth V. Gangashetty, International Institute of Information Technology Hyderabad, India 
SP-P19.4: ON THE RECOVERY OF TIME-VARYING SPECTRAL ENVELOPE 5400 INFORMATION FROM AQHM-DERIVED SPECTRA

Yannis Agiomyrgiannakis, Yannis Stylianou, ICS - Foundation for Research \& Technology, Greece

SP-P19.5: A TIME-WARPING FRAMEWORK FOR SPEECH TURBULENCE-NOISE 5404 COMPONENT ESTIMATION DURING APERIODIC PHONATION

Nicolas Malyska, Thomas Quatieri, MIT Lincoln Laboratory, United States

SP-P19.6: GLOTTAL INVERSE FILTERING USING STABILISED WEIGHTED LINEAR 5408 PREDICTION

George Kafentzis, Yannis Stylianou, University of Crete, Greece; Paavo Alku, Aalto University, Finland

SP-P19.7: EFFICIENT IMPLEMENTATION OF PROBABILISTIC MULTI-PITCH TRACKING 5412

Michael Wohlmayr, Robert Peharz, Franz Pernkopf, Graz University of Technology, Austria

SP-P19.8: GAIN-ROBUST MULTI-PITCH TRACKING USING SPARSE NONNEGATIVE 5416 MATRIX FACTORIZATION

Robert Peharz, Michael Wohlmayr, Franz Pernkopf, Graz University of Technology, Austria

SP-P19.9: AN INTERFERENCE-FREE REPRESENTATION OF INSTANTANEOUS 5420 FREQUENCY OF PERIODIC SIGNALS AND ITS APPLICATION TO FO EXTRACTION

Hideki Kawahara, Toshio Irino, Wakayama University, Japan; Masanori Morise, Ritsumeikan University, Japan

SP-P19.10: ROBUST SPEECH REPRESENTATION OF VOICED SOUNDS BASED ON 5424 SYNCHRONY DETERMINATION WITH PLLS

Patricia Pelle, University of Buenos Aires, Argentina; Horacio Franco, SRI International, United States; Claudio Estienne, University of Buenos Aires, Argentina

SP-P19.11: SIMULATION OF HEARING LOSS USING COMPRESSIVE GAMMACHIRP 5428 AUDITORY FILTERS

Hongmei Hu, Jinqiu Sang, Mark Lutman, Stefan Bleeck, Southampton University, United Kingdom

\section{SP-P20: SPEAKER RECOGNITION II}

SP-P20.1: STRUCTURAL MAP ADAPTATION IN GMM-SUPERVECTOR BASED SPEAKER 5432 RECOGNITION

Marc Ferras, Furui Laboratory, Japan; Koichi Shinoda, Shinoda Laboratory, Japan; Sadaoki Furui, Furui Laboratory, Japan

SP-P20.2: A NEW SPEAKER IDENTIFICATION ALGORITHM FOR GAMING SCENARIOS 5436

Hoang Do, Brown University, United States; Ivan Tashev, Alex Acero, Microsoft Corporation, United States

SP-P20.3: A COCHLEAR NEURON BASED ROBUST FEATURE FOR SPEAKER 5440 RECOGNITION

Datao You, Tao Jiang, Jiqing Han, Tieran Zheng, Harbin Institute of Technology, China

SP-P20.4: SURVEY AND EVALUATION OF ACOUSTIC FEATURES FOR SPEAKER 5444 RECOGNITION

Aaron Lawson, Pavel Vabishchevich, RADC Inc., United States; Mark Huggins, Oasis Systems, United States; Paul Ardis, Brandon Battles, Allen Stauffer, RADC Inc., United States

SP-P20.5: HILBERT ENVELOPE BASED FEATURES FOR ROBUST SPEAKER 5448 IDENTIFICATION UNDER REVERBERANT MISMATCHED CONDITIONS

Seyed Omid Sadjadi, John H.L. Hansen, The University of Texas at Dallas, United States

SP-P20.6: USING CLUSTERING COMPARISON MEASURES FOR SPEAKER 5452 RECOGNITION

Jia Min Karen Kua, Julien Epps, Mohaddeseh Nosratighods, Eliathamby Ambikairajah, The University of New South Wales, Australia; Eric H. C. Choi, National ICT Australia, Australia 
RECOGNITION USING I-VECTORS

Mitchell McLaren, David van Leeuwen, Radboud University Nijmegen, Netherlands

SP-P20.8: IMPROVED SPEAKER RECOGNITION WHEN USING I-VECTORS FROM 5460 MULTIPLE SPEECH SOURCES

Mitchell McLaren, David van Leeuwen, Radboud University Nijmegen, Netherlands

SP-P20.9: LOQUENDO - POLITECNICO DI TORINO'S 2010 NIST SPEAKER 5464 RECOGNITION EVALUATION SYSTEM

Fabio Castaldo, Daniele Colibro, Claudio Vair, Loquendo S.p.A, Italy; Sandro Cumani, Pietro Laface, Politecnico di Torino, Italy

SP-P20.10: ROBUST SPEAKER IDENTIFICATION USING A CASA FRONT-END 5468

Xiaojia Zhao, Yang Shao, DeLiang Wang, The Ohio State University, United States

\section{SP-P21: ROBUST ASR III}

SP-P21.1: ON-LINE MEMORY-BASED PARAMETRIC EQUALIZATION TO MULTIMODAL 5472 TRAINING CONDITIONS

Roberto Gemello, Franco Mana, Loquendo, Italy; Luz Garcia, Josè Carlos Segura, University of Granada, Spain

SP-P21.2: COMPENSATION OF PARTLY RELIABLE COMPONENTS FOR BAND-LIMITED 5476 SPEECH RECOGNITION WITH MISSING DATA TECHNIQUES

Yongjun He, Jiqing Han, Tieran Zheng, Guibin Zheng, Harbin Institute of Technology, China

SP-P21.3: MAXIMUM LIKELIHOOD ADAPTATION OF HISTOGRAM EQUALIZATION WITH 5480 CONSTRAINT FOR ROBUST SPEECH RECOGNITION

Xiong Xiao, Nanyang Technological University, Singapore; Jinyu Li, Microsoft Corporation, United States; Eng Siong Chng, Nanyang Technological University, Singapore; Haizhou Li, Institute for Infocomm Research, Singapore

SP-P21.4: FRAME-WISE HMM ADAPTATION USING STATE-DEPENDENT 5484 REVERBERATION ESTIMATES

Armin Sehr, Roland Maas, Walter Kellermann, University of Erlangen-Nuremberg, Germany

SP-P21.5: AN ITERATIVE LEAST-SQUARES TECHNIQUE FOR DEREVERBERATION 5488

Kshitiz Kumar, Bhiksha Raj, Rita Singh, Richard Stern, Carnegie Mellon University, United States

SP-P21.6: AMPLITUDE MODULATION SPECTROGRAM BASED FEATURES FOR ROBUST 5492 SPEECH RECOGNITION IN NOISY AND REVERBERANT ENVIRONMENTS

Niko Moritz, Fraunhofer IDMT / Project Group HSA, Germany; Jörn Anemüller, Birger Kollmeier, Carl-von-Ossietzky University Oldenburg, Germany

SP-P21.7: COMBINING SPEAKER AND NOISE FEATURE NORMALIZATION TECHNIQUES 5496 FOR AUTOMATIC SPEECH RECOGNITION

Luz García Martínez, M. Carmen Benítez Ortúzar, Jose Carlos Segura Luna, University of Granada, Spain; S. Umesh, Indian Institute of Technology Madras, India

SP-P21.8: RAPID JOINT SPEAKER AND NOISE COMPENSATION FOR ROBUST SPEECH 5500 RECOGNITION

K. K. Chin, Haitian Xu, Mark J. F. Gales, Catherine Breslin, Kate Knill, Cambridge Research Laboratory / Toshiba Research Europe Limited, United Kingdom

SP-P21.9: A SAMPLING-BASED ENVIRONMENT POPULATION PROJECTION APPROACH 5504 FOR RAPID ACOUSTIC MODEL ADAPTATION

Yu Tsao, Shigeki Matsuda, Shinsuke Sakai, Ryosuke Isotani, Hisashi Kawai, Satoshi Nakamura, National Institute of Information and Communications Technology, Japan 
SP-P21.10: MULTI-MICROPHONE INTERFERENCE SUPPRESSION USING THE

PRINCIPAL SUBSPACE MODIFICATION AND ITS APPLICATION TO SPEECH RECOGNITION

Gibak Kim, Daegu University, Republic of Korea

SP-P21.11: MITIGATION OF REVERBERATION ON SPEAKER IDENTIFICATION VIA 5512 HOMOMORPHIC FILTERING OF THE LINEAR PREDICTION RESIDUAL

Catherine Vannicola, Oasis Systems, United States; Brett Smolenski, Brandon Battles, Paul Ardis, RADC Inc., United States

\section{SLP-L1: LANGUAGE MODELING II}

SLP-L1.1: MULTI-CLASS MODEL M 5516

Ahmad Emami, Stanley Chen, IBM T.J. Watson Research Center, United States

SLP-L1.2: DISTRIBUTED TRAINING OF LARGE SCALE EXPONENTIAL LANGUAGE 5520 MODELS

Abhinav Sethy, Stanley Chen, Bhuvana Ramabhadran, IBM, United States

SLP-L1.3: STRUCTURED OUTPUT LAYER NEURAL NETWORK LANGUAGE MODEL

Hai Son Le, LIMSI CNRS / Uni. Paris-Sud, France; Ilya Oparin, LIMSI CNRS, France; Alexandre Allauzen, LIMSI CNRS / Uni.

Paris-Sud, France; Jean-Luc Gauvain, LIMSI CNRS, France; Francois Yvon, LIMSI CNRS / Uni. Paris-Sud, France

SLP-L1.4: EXTENSIONS OF RECURRENT NEURAL NETWORK LANGUAGE MODEL 5528

Tomáš Mikolov, Stefan Kombrink, Lukas Burget, Jan Cernocky, Brno University of Technology, Czech Republic; Sanjeev

Khudanpur, The Johns Hopkins University, United States

SLP-L1.5: VARIATIONAL APPROXIMATION OF LONG-SPAN LANGUAGE MODELS FOR 5532 LVCSR

Anoop Deoras, Center for Language and Speech Processing, United States; Tomáš Mikolov, Stefan Kombrink, Martin Karafiát, Brno University of Technology, Czech Republic; Sanjeev Khudanpur, Center for Language and Speech Processing, United States

\section{SLP-L2: SPOKEN DOCUMENT PROCESSING}

SLP-L2.1: AUTOMATIC MINUTE GENERATION FOR PARLIAMENTARY SPEECH USING 5536 CONDITIONAL RANDOM FIELDS

Jian Zhang, Pascale Fung, Ho Yin Chan, The Hong Kong University of Science and Technology, Hong Kong SAR of China

SLP-L2.2: CONCEPT-BASED CLASSIFICATION FOR MULTI-DOCUMENT 5540 SUMMARIZATION

Asli Celikyilmaz, University of California Berkeley, United States; Dilek Hakkani-Tür, Microsoft Corporation, United States

SLP-L2.3: USING LATENT TOPIC FEATURES TO IMPROVE BINARY CLASSIFICATION OF 5544 SPOKEN DOCUMENTS

Jonathan Wintrode, The Johns Hopkins University, United States

SLP-L2.4: A SEGMENT-LEVEL CONFIDENCE MEASURE FOR SPOKEN DOCUMENT 5548 RETRIEVAL

Gregory Senay, Georges Linarès, Benjamin Lecouteux, University of Avignon, France

SLP-L2.5: HANDLING VERBOSE QUERIES FOR SPOKEN DOCUMENT RETRIEVAL

Shih-Hsiang Lin, National Taiwan Normal University, Taiwan; Ea-Ee Jan, IBM T.J. Watson Research Center, United States;

Berlin Chen, National Taiwan Normal University, Taiwan

SLP-L2.6: AUTOMATIC IDENTIFICATION OF SPEAKER ROLE AND 5556 AGREEMENT/DISAGREEMENT IN BROADCAST CONVERSATION

Wen Wang, SRI International, United States; Sibel Yaman, ICSI, United States; Kristin Precoda, Colleen Richey, SRI International, United States 
SLP-P1.1: GENERATING COMPOUND WORDS WITH HIGH ORDER N-GRAM 5560

INFORMATION IN LARGE VOCABULARY SPEECH RECOGNITION SYSTEMS

Jie Zhou, Qin Shi, Yong Qin, IBM, China

SLP-P1.2: BAYESIAN CLASS-BASED LANGUAGE MODELS 5564

Yi Su, Nuance Communications Inc., Canada

SLP-P1.3: RELEVANCE LANGUAGE MODELING FOR SPEECH RECOGNITION 5568

Kuan-Yu Chen, Berlin Chen, National Taiwan Normal University, Taiwan

SLP-P1.4: NAMED ENTITY RECOGNITION FROM CONVERSATIONAL TELEPHONE 5572 SPEECH LEVERAGING WORD CONFUSION NETWORKS FOR TRAINING AND RECOGNITION

Gakuto Kurata, Nobuyasu Itoh, Masafumi Nishimura, IBM Japan, Japan; Abhinav Sethy, Bhuvana Ramabhadran, IBM, United States

SLP-P1.5: TRAINING OF ERROR-CORRECTIVE MODEL FOR ASR WITHOUT USING 5576 AUDIO DATA

Gakuto Kurata, Nobuyasu Itoh, Masafumi Nishimura, IBM Japan, Japan

SLP-P1.6: SUBSEQUENCE SIMILARITY LANGUAGE MODELS

Juan Huerta, IBM T.J. Watson Research Center, United States

SLP-P1.7: EXTRACTING CALL-REASON SEGMENTS FROM CONTACT CENTER DIALOGS 5584 BY USING AUTOMATICALLY ACQUIRED BOUNDARY EXPRESSIONS

Takaaki Fukutomi, Satoshi Kobashikawa, Taichi Asami, Tsubasa Shinozaki, Hirokazu Masataki, Satoshi Takahashi, NTT

Corporation, Japan

SLP-P1.8: ROUND-ROBIN DUEL DISCRIMINATIVE LANGUAGE MODELS IN ONE-PASS 5588 DECODING WITH ON-THE-FLY ERROR CORRECTION

Takanobu Oba, Takaaki Hori, NTT Corporation, Japan; Akinori Ito, Tohoku University, Japan; Atsushi Nakamura, NTT

Corporation, Japan

SLP-P1.9: POMDP CONCEPT POLICIES AND TASK STRUCTURES FOR HYBRID DIALOG MANAGEMENT

Sebastian Varges, Giuseppe Riccardi, Silvia Quarteroni, Alexei V. Ivanov, University of Trento, Italy

SLP-P1.10: SIMULTANEOUS DIALOG ACT SEGMENTATION AND CLASSIFICATION FROM 5596 HUMAN-HUMAN SPOKEN CONVERSATIONS

Silvia Quarteroni, Alexei V. Ivanov, Giuseppe Riccardi, University of Trento, Italy

SLP-P1.11: A SINGLE-PORT NON-PARAMETRIC MODEL OF TURN-TAKING IN 5600 MULTI-PARTY CONVERSATION

Kornel Laskowski, Jens Edlund, Mattias Heldner, KTH Speech, Music and Hearing, Sweden

\section{SLP-P2: SPEECH TRANSLATION AND SEMANTIC CLASSIFICATION}

SLP-P2.1: LEXICON MODELING FOR QUERY UNDERSTANDING. 5604

Jingjing Liu, Massachusetts Institute of Technology, United States; Xiao Li, Alex Acero, Ye-Yi Wang, Microsoft Research, United States

SLP-P2.2: A NOVEL DECISION FUNCTION AND THE ASSOCIATED DECISION-FEEDBACK 5608 LEARNING FOR SPEECH TRANSLATION

Yaodong Zhang, Massachusetts Institute of Technology, United States; Li Deng, Xiaodong He, Alex Acero, Microsoft Research, United States 
SLP-P2.3: COMBINATION OF STOCHASTIC UNDERSTANDING AND MACHINE

TRANSLATION SYSTEMS FOR LANGUAGE PORTABILITY OF DIALOGUE SYSTEMS

Bassam Jabaian, Laurent Besacier, LIG / University Joseph Fourier, France; Fabrice Lefèvre, LIA / University of Avignon,

France

SLP-P2.4: SEMANTIC DATA SELECTION FOR VERTICAL BUSINESS VOICE SEARCH 5616

Giuseppe Di Fabbrizio, Diamantino Caseiro, Amanda Stent, AT\&T Labs Research, United States

SLP-P2.5: A CONDITIONAL MODEL FOR TRIGGERING UNDERSTANDING ACTIONS IN A 5620 SPEECH UNDERSTANDING SYSTEM

Frédéric Duvert, Renato De Mori, University of Avignon, France

SLP-P2.6: BILINGUAL AUDIO-SUBTITLE EXTRACTION USING AUTOMATIC 5624 SEGMENTATION OF MOVIE AUDIO

Andreas Tsiartas, Prasanta Ghosh, Panayiotis Georgiou, Shrikanth S. Narayanan, University of Southern California, United States

SLP-P2.7: SENTENCE SIMPLIFICATION FOR SPOKEN LANGUAGE UNDERSTANDING 5628

Gokhan Tur, Dilek Hakkani-Tür, Larry Heck, Speech at Microsoft | Microsoft Research, United States; S. Parthasarathy,

Microsoft Corporation, United States

SLP-P2.8: WHY WORD ERROR RATE IS NOT A GOOD METRIC FOR SPEECH 5632 RECOGNIZER TRAINING FOR THE SPEECH TRANSLATION TASK?

Xiaodong He, Li Deng, Alex Acero, Microsoft Research, United States

SLP-P2.9: EXPLOITING QUERY CLICK LOGS FOR UTTERANCE DOMAIN DETECTION 5636 IN SPOKEN LANGUAGE UNDERSTANDING

Dilek Hakkani-Tür, Larry Heck, Gokhan Tur, Microsoft Corporation, United States

SLP-P2.10: TOWARDS ROBUST WORD DISCOVERY BY SELF-SIMILARITY MATRIX 5640 COMPARISON

Armando Muscariello, Guillaume Gravier, Frédéric Bimbot, IRISA (CNRS\&INRIA) Metiss: Speech and Audio Processing

Research Group, France

\section{SLP-P3: SPOKEN TERM DETECTION AND LANGUAGE UNDERSTANDING}

SLP-P3.1: IMPROVED SPOKEN TERM DETECTION WITH GRAPH-BASED RE-RANKING 5644 IN FEATURE SPACE

Yun-Nung Chen, Chia-Ping Chen, Hung-Yi Lee, Chun-An Chan, Lin-Shan Lee, National Taiwan University, Taiwan

SLP-P3.2: IMPROVED SPOKEN TERM DETECTION USING SUPPORT VECTOR 5648 MACHINES BASED ON LATTICE CONTEXT CONSISTENCY

Hung-yi Lee, Tsung-wei Tu, Chia-ping Chen, Chao-yu Huang, Lin-Shan Lee, National Taiwan University, Taiwan

SLP-P3.3: INTEGRATING FRAME-BASED AND SEGMENT-BASED DYNAMIC TIME 5652

WARPING FOR UNSUPERVISED SPOKEN TERM DETECTION WITH SPOKEN QUERIES

Chun-an Chan, Lin-Shan Lee, National Taiwan University, Taiwan

SLP-P3.4: HANDLING OVERLAPS IN SPOKEN TERM DETECTION

Dong Wang, Nicholas Evans, Raphaël Troncy, EURECOM, France; Simon King, University of Edinburgh, United Kingdom

SLP-P3.5: AN INNER-PRODUCT LOWER-BOUND ESTIMATE FOR DYNAMIC TIME 5660 WARPING

Yaodong Zhang, James Glass, Massachusetts Institute of Technology, United States

SLP-P3.6: EFFICIENT OUT-OF-VOCABULARY TERM DETECTION BY N-GRAM ARRAY 5664 INDICES WITH DISTANCE FROM A SYLLABLE LATTICE

Keisuke Iwami, Yasuhisa Fujii, Kazumasa Yamamoto, Seiichi Nakagawa, Toyohashi University of Technology, Japan 


\section{NORMALIZATION FOR GLDS-SVM BASED AUTOMATIC MISPRONUNCIATION DETECTION}

\section{METHOD}

Hongyan Li, Shen Huang, Shijin Wang, Jiaen Liang, Bo Xu, Institute of Automation / Chinese Academy of Sciences, China

SLP-P3.8: A NATIVENESS CLASSIFIER FOR TED TALKS 5672

José Lopes, Isabel Trancoso, INESC / Instituto Superior Técnico, Portugal; Alberto Abad, INESC, Portugal

SLP-P3.9: A PAIRED TEST FOR RECOGNIZER SELECTION WITH UNTRANSCRIBED 5676

DATA

Bhiksha Raj, Rita Singh, James Baker, Carnegie Mellon University, United States

SLP-P3.10: DEEP BELIEF NETS FOR NATURAL LANGUAGE CALL-ROUTING

Ruhi Sarikaya, IBM T.J. Watson Research Center, United States; Geoffrey Hinton, University of Toronto, Canada; Bhuvana Ramabhadran, IBM T.J. Watson Research Center, United States

SLP-P3.11: ROBUST SPEAKER TURN ROLE LABELING OF TV BROADCAST NEWS 5684 SHOWS

Geraldine Damnati, Delphine Charlet, Orange Labs, France

\section{SLP-P4: PARALINGUISTIC AND NON-LINGUISTIC FEATURES}

SLP-P4.1: DEEP NEURAL NETWORKS FOR ACOUSTIC EMOTION RECOGNITION: 5688 RAISING THE BENCHMARKS

André Stuhlsatz, Christine Meyer, Duesseldorf University of Applied Sciences, Germany; Florian Eyben, Technische Universitaet Muenchen, Germany; Thomas Zielke, Guenter Meier, Duesseldorf University of Applied Sciences, Germany; Björn Schuller,

Technische Universität München, Germany

SLP-P4.2: ITERATIVE FEATURE NORMALIZATION FOR EMOTIONAL SPEECH 5692 DETECTION

Carlos Busso, The University of Texas at Dallas, United States; Angeliki Metallinou, Shrikanth S. Narayanan, University of Southern California, United States

SLP-P4.3: EXPERIMENTS IN CONTEXT-INDEPENDENT RECOGNITION OF 5696 NON-LEXICAL 'YES' OR 'NO' RESPONSES

Shiva Sundaram, Robert Schleicher, Nathalie Diehl, Deutsche Telekom Laboratories, Germany

SLP-P4.4: VOICE SOURCE FEATURES FOR COGNITIVE LOAD CLASSIFICATION

Tet Fei Yap, Julien Epps, Eliathamby Ambikairajah, The University of New South Wales, Australia; Eric H. C. Choi, National ICT Australia, Australia

SLP-P4.5: PERCEPTUAL DIFFERENTIATION MODELING EXPLAINS PHONEME 5704 MISPRONUNCIATION BY NON-NATIVE SPEAKERS

Christos Koniaris, Olov Engwall, KTH - Royal Institute of Technology, Sweden

SLP-P4.6: ALLOPHONIC VARIATIONS IN VISUAL SPEECH SYNTHESIS FOR 5708 CORRECTIVE FEEDBACK IN CAPT

Ka Ho Wong, Wai Kit Lo, Helen Meng, The Chinese University of Hong Kong, Hong Kong SAR of China

SLP-P4.7: ROLE OF NUCLEUS BASED CONTEXT IN WORD-INDEPENDENT SYLLABLE 5712 STRESS CLASSIFICATION

Harish Doddala, Om D Deshmukh, Ashish Verma, IBM Research - India, India

SLP-P4.8: GAUSSIAN MIXTURE MODELING OF VOWEL DURATIONS FOR AUTOMATED 5716 ASSESSMENT OF NON-NATIVE SPEECH

Xie Sun, University of Missouri, United States; Keelan Evanini, Educational Testing Service, United States 
LEVEL AUDIO AND VISUAL FEATURES

Vijay Chandrasekhar, Stanford University, United States; Mehmet Emre Sargin, David A. Ross, Google Inc., United States

SLP-P4.11: LOCATION-AWARE QUERY PARSING FOR MOBILE VOICE SEARCH. 5728

Junlan Feng, AT\&T Labs Research, United States

\section{SS-L1: SIGNAL PROCESSING METHODS FOR FINANCE APPLICATIONS}

SS-L1.1: EMPIRICAL EVIDENCE AGAINST CAPM: RELATING ALPHAS AND RETURNS TO 5732 BETAS

Mayur Agrawal, Debabrata Mohapatra, Ilya Pollak, Purdue University, United States

SS-L1.2: RISK MANAGEMENT FOR TRADING IN MULTIPLE FREQUENCIES 5736 Mustafa Torun, Ali Akansu, New Jersey Institute of Technology, United States; Marco Avellaneda, New York University, United States

SS-L1.3: MODELING MICROSTRUCTURE NOISE USING HAWKES PROCESSES 5740

Emmanuel Bacry, Centre de Mathématiques Appliquées, France; Sylvain Delattre, Université Paris Diderot, France; Marc Hoffmann, CREST, France; Jean-François Muzy, Université de Corse, France

SS-L1.4: SIGNAL EXTRAPOLATION USING EMPIRICAL MODE DECOMPOSITION WITH 5744 FINANCIAL APPLICATIONS

Nikolaos Tsakalozos, Konstantinos Drakakis, Scott Rickard, University College Dublin, Ireland

SS-L1.5: FACTOR GRAPH SWITCHING PORTFOLIOS UNDER TRANSACTION COSTS 5748

Andrew Bean, Andrew Singer, University of Illinois Urbana-Champaign, United States

SS-L1.6: COMPARISON OF SEVERAL COVARIANCE MATRIX ESTIMATORS FOR 5752 PORTFOLIO OPTIMIZATION

Ka Ki Ng, Priyanka Agarwal, Nathan Mullen, Dzung Du, Ilya Pollak, Purdue University, United States

\section{SS-L2: MEDICAL IMAGING}

SS-L2.1: SIGNAL PROCESSING IN MEDICAL IMAGING AND IMAGE-GUIDED 5756 INTERVENTION

Milan Sonka, The University of Iowa, United States

SS-L2.2: EFFICIENT IMAGE RECONSTRUCTION UNDER SPARSITY CONSTRAINTS 5760 WITH APPLICATION TO MRI AND BIOLUMINESCENCE TOMOGRAPHY

Matthieu Guerquin-Kern, Jean-Charles Baritaux, Michael Unser, Ecole Polytechnique Fédérale de Lausanne, Switzerland

SS-L2.3: THE MEANING OF INTERIOR TOMOGRAPHY

Ge Wang, Virginia Polytechnic Institute and State University, United States

SS-L2.4: TOWARDS INTEGRATED ANALYSIS OF LONGITUDINAL WHOLE-BODY SMALL 5768 ANIMAL IMAGING STUDIES

Boudewijn Lelieveldt, Leiden University Medical Center, Netherlands; Charl Botha, Delft University of Technology, Netherlands; Eric Kaijzel, Leiden University Medical Center, Netherlands; Emile Hendriks, Delft University of Technology, Netherlands; Johan Reiber, Clemens Lowik, Jouke Dijkstra, Leiden University Medical Center, Netherlands

SS-L2.5: TIME-VARYING LUNG VENTILATION ANALYSIS OF 4DCT USING IMAGE 5772 REGISTRATION

Kai Ding, University of Virginia, United States; Kaifang Du, Kunlin Cao, Gary Christensen, Joseph Reinhardt, University of Iowa, United States 


\section{SS-L3: BIO-INSPIRED INFORMATION PROCESSING AND NETWORKS}

SS-L3.1: BIO-INSPIRED SWARMING MODELS FOR DECENTRALIZED RADIO ACCESS 5780 INCORPORATING RANDOM LINKS AND QUANTIZED COMMUNICATIONS

Paolo Di Lorenzo, Sergio Barbarossa, University of Rome La Sapienza, Italy

SS-L3.2: SOCIAL NORM AND LONG-RUN LEARNING IN PEER-TO-PEER NETWORKS 5784

Yu Zhang, Mihaela van der Schaar, University of California Los Angeles, United States

SS-L3.3: BIO-INSPIRED COOPERATIVE OPTIMIZATION WITH APPLICATION TO 5788 BACTERIA MOTILITY

Jianshu Chen, Ali H. Sayed, University of California Los Angeles, United States

SS-L3.4: EMERGENCE OF RATIONALITY AMONGST SIMPLE NODES PERFORMING 5792 ADAPTIVE FILTERING

Vikram Krishnamurthy, Omid Namvar Gharehshiran, University of British Columbia, Canada; Amir Danak, McGill University, Canada

SS-L3.5: GLOBAL EMERGENT BEHAVIORS IN CLOUDS OF AGENTS 5796

Soummya Kar, Princeton University, United States; José M.F. Moura, Carnegie Mellon University, United States

SS-L3.6: DIFFUSIONS OF INNOVATIONS ON DETERMINISTIC TOPOLOGIES. 5800

Ercan Yildiz, Massachusetts Institute of Technology, United States; Daron Acemoglu, MIT ECON, United States; Asuman

Ozdaglar, Massachusetts Institute of Technology, United States; Anna Scaglione, University of California Davis, United States

\section{SS-L4: LEARNING LOW-DIMENSIONAL MODELS FOR LARGE-SCALE DATA}

SS-L4.1: COSPARSE ANALYSIS MODELING - UNIQUENESS AND ALGORITHMS. 5804

Sangnam Nam, IRISA / INRIA Rennes - Bretagne Atlantique, France; Michael Davies, University of Edinburgh, United Kingdom; Michael Elad, The Technion - Israel Institute of Technology, Israel; Rémi Gribonval, IRISA / INRIA Rennes - Bretagne Atlantique, France

SS-L4.2: AN ALPS VIEW OF SPARSE RECOVERY. 5808

Volkan Cevher, Ecole Polytechnique Fédérale de Lausanne, Switzerland

SS-L4.3: DICTIONARY LEARNING OF CONVOLVED SIGNALS.

Daniele Barchiesi, Mark D. Plumbley, Queen Mary University of London, United Kingdom

SS-L4.4: COLLABORATIVE SOURCES IDENTIFICATION IN MIXED SIGNALS VIA 5816 HIERARCHICAL SPARSE MODELING

Pablo Sprechmann, Ignacio Ramirez, University of Minnesota, United States; Pablo Cancela, Universidad de la Republica, Uruguay; Guillermo Sapiro, University of Minnesota, United States

SS-L4.5: DENOISING OF IMAGE PATCHES VIA SPARSE REPRESENTATIONS WITH 5820 LEARNED STATISTICAL DEPENDENCIES

Tomer Faktor, Yonina C. Eldar, Michael Elad, Technion / Israel Institute of Technology, Israel

SS-L4.6: COVARIATE-DEPENDENT DICTIONARY LEARNING AND SPARSE CODING 5824

Mingyuan Zhou, Hongxia Yang, Duke University, United States; Guillermo Sapiro, University of Minnesota, United States; David Dunson, Lawrence Carin, Duke University, United States

\section{SS-L5: AUDIO/VISUAL DETECTION OF NON-LINGUISTIC VOCAL OUTBURSTS}

SS-L5.1: ASSOCIATING CHILDREN'S NON-VERBAL AND VERBAL BEHAVIOUR: BODY 5828 MOVEMENTS, EMOTIONS, AND LAUGHTER IN A HUMAN-ROBOT INTERACTION Anton Batliner, Stefan Steidl, Elmar Nöth, Universität Erlangen-Nürnberg, Germany 
Nick Campbell, John Kane, University of Dublin, Ireland; Helena Moniz, FLUL/INESC-ID, Ireland

SS-L5.3: ONLINE DETECTION OF VOCAL LISTENER RESPONSES WITH MAXIMUM 5836

\section{LATENCY CONSTRAINTS}

Daniel Neiberg, KTH - Royal Institute of Technology, Sweden; Khiet P. Truong, University of Twente, Netherlands

SS-L5.4: LOCALIZATION OF NON-LINGUISTIC EVENTS IN SPONTANEOUS SPEECH BY 5840 NON-NEGATIVE MATRIX FACTORIZATION AND LONG SHORT-TERM MEMORY

Felix Weninger, Björn Schuller, Martin Wöllmer, Gerhard Rigoll, Technische Universität München, Germany

SS-L5.5: AUDIOVISUAL CLASSIFICATION OF VOCAL OUTBURSTS IN HUMAN 5844 CONVERSATION USING LONG-SHORT-TERM MEMORY NETWORKS

Florian Eyben, Technische Universitaet Muenchen, Germany; Stavros Petridis, Imperial College London, United Kingdom; Björn Schuller, Technische Universität München, Germany; George Tzimiropoulos, Stefanos Zafeiriou, Maja Pantic, Imperial College London, United Kingdom

SS-L5.6: PANEL DISCUSSION. $.1 \square$

Björn Schuller, Technische Universität München, Germany

\section{SS-L6: SECURE SIGNAL PROCESSING}

SS-L6.1: IS MULTIPARTY COMPUTATION ANY GOOD IN PRACTICE?. 5848

Claudio Orlandi, Aarhus University, Denmark

SS-L6.2: ANALYSIS OF THE SECURITY OF LINEAR BLINDING TECHNIQUES FROM AN 5852 INFORMATION THEORETICAL POINT OF VIEW

Tiziano Bianchi, Alessandro Piva, University of Firenze, Italy; Mauro Barni, University of Siena, Italy

SS-L6.3: SECURE VIDEO PROCESSING: PROBLEMS AND CHALLENGES 5856

Wenjun Lu, Avinash Varna, Min Wu, University of Maryland College Park, United States

SS-L6.4: EFFICIENT PROTOCOLS FOR SECURE ADAPTIVE FILTERING 5860

Juan Ramón Troncoso-Pastoriza, Fernando Pérez-González, University of Vigo, Spain

SS-L6.5: EFFICIENTLY COMPUTING PRIVATE RECOMMENDATIONS 5864

Zekeriya Erkin, Michael Beye, Thijs Veugen, Reginald L. Lagendijk, Delft University of Technology, Netherlands

SS-L6.6: PRIVACY PRESERVING PROBABILISTIC INFERENCE WITH HIDDEN MARKOV 5868 MODELS

Manas Pathak, Carnegie Mellon University, United States; Shantanu Rane, Wei Sun, Mitsubishi Electric Research Laboratories, United States; Bhiksha Raj, Carnegie Mellon University, United States

\section{SS-L7: INNOVATIVE REPRESENTATIONS OF AUDIO}

SS-L7.1: SPEECH PROCESSING WITH A CORTICAL REPRESENTATION OF AUDIO 5872

Nima Mesgarani, Shihab Shamma, University of Maryland, United States

SS-L7.2: SPARSE CODING OF AUDITORY FEATURES FOR MACHINE HEARING IN 5876 INTERFERENCE

Richard Lyon, Jay Ponte, Gal Chechik, Google Inc., United States

SS-L7.3: CLASSIFYING SOUNDTRACKS WITH AUDIO TEXTURE FEATURES 5880

Daniel P.W. Ellis, Xiaohong Zeng, Columbia University, United States; Josh McDermott, New York University, United States

SS-L7.4: LEARNING A BETTER REPRESENTATION OF SPEECH SOUND WAVES USING 5884 RESTRICTED BOLTZMANN MACHINES

Navdeep Jaitly, Geoffrey Hinton, University of Toronto, Canada 
Paris Smaragdis, University of Illinois Urbana-Champaign, United States

\section{SS-L8: HUMAN ASSISTED SPEAKER RECOGNITION}

SS-L8.1: INCLUDING HUMAN EXPERTISE IN SPEAKER RECOGNITION SYSTEMS: 5896 REPORT ON A PILOT EVALUATION

Craig Greenberg, Alvin Martin, National Institute of Standards and Technology, United States; George Doddington, N/A, United States; John Godfrey, US Department of Defense, United States

SS-L8.2: FORENSIC VOICE COMPARISON WITH SECULAR SHIBBOLETHS - A HYBRID 5900 FUSED GMM-MULTIVARIATE LIKELIHOOD RATIO-BASED APPROACH USING ALVEOLOPALATAL FRICATIVE CEPSTRAL SPECTRA

Phil Rose, Australian National University, Australia

SS-L8.3: USSS-MITLL 2010 HUMAN ASSISTED SPEAKER RECOGNITION 5904 Reva Schwartz, United States Secret Service, United States; Joseph Campbell, Wade Shen, Douglas Sturim, William Campbell, Fred Richardson, Robert Dunn, Robert Granville, MIT Lincoln Laboratory, United States

SS-L8.4: CALIBRATION AND WEIGHT OF THE EVIDENCE BY HUMAN LISTENERS. THE 5908 ATVS-UAM SUBMISSION TO NIST HUMAN-AIDED SPEAKER RECOGNITION 2010

Daniel Ramos, Javier Franco-Pedroso, Joaquin Gonzalez-Rodriguez, Universidad Autonoma de Madrid, Spain

SS-L8.5: SPEAKER VERIFICATION BY INEXPERIENCED AND EXPERIENCED 5912 LISTENERS VS. SPEAKER VERIFICATION SYSTEM

Juliette Kahn, University of Avignon, France; Nicolas Audibert, Laboratoire de Phonétique et Phonologie, France; Solange Rossato, University of Grenoble, France; Jean-François Bonastre, University of Avignon, France

SS-L8.6: ASSESSING THE SPEAKER RECOGNITION PERFORMANCE OF NAIVE 5916 LISTENERS USING MECHANICAL TURK

Wade Shen, Joseph Campbell, Derek Straub, Massachusetts Institute of Technology / MIT Lincoln Laboratory, United States; Reva Schwartz, USSS, United States

\section{SS-L9: PARTICLE FILTERING FOR HIGH DIMENSIONAL PROBLEMS}

SS-L9.1: PARTICLE FLOW FOR NONLINEAR FILTERS

Fred Daum, Jim Huang, Raytheon, United States

SS-L9.2: NON-PARAMETRIC BAYESIAN MEASUREMENT NOISE DENSITY ESTIMATION 5924 IN NON-LINEAR FILTERING

Emre Özkan, Saikat Saha, Fredrik Gustafsson, Linköping University, Sweden; Vaclav Smidl, Institute of Information Theory and Automation, Czech Republic

SS-L9.3: NON-CENTRALIZED TARGET TRACKING WITH MOBILE AGENTS . 5928

Petar Djuric, Jonathan Beaudeau, Monica Bugallo, Stony Brook University, United States

SS-L9.4: PARTICLE ALGORITHMS FOR FILTERING IN HIGH DIMENSIONAL STATE 5932 SPACES: A CASE STUDY IN GROUP OBJECT TRACKING

Lyudmila Mihaylova, Lancaster University, United Kingdom; Avishy Carmi, Asher Space Research Institute, Technion, Israel 
SIGNATURES USING PARTICLE FILTERS

Mehmet Guldogan, Fredrik Gustafsson, Umut Orguner, Linköping University, Sweden; Svante Bjorklund, Henrik Petersson,

Amer Nezirovic, Swedish Defence Research Agency, Sweden

\section{SS-L10: TOMORROW'S SMART GRIDS}

SS-L10.1: MODELING NODAL PRICES IN DEREGULATED ELECTRICITY MARKETS IN 5944 THE USA: CURRENT PRACTICES AND FUTURE NEEDS

Tim Mount, Cornell University, United States

SS-L10.2: ACCELERATING STANDARDS AND MEASUREMENTS FOR THE SMART GRID. 5948

David Wollman, National Institute of Standards and Technology, United States

SS-L10.3: MALICIOUS DATA ATTACK ON REAL-TIME ELECTRICITY MARKET 5952

Liyan Jia, Robert J. Thomas, Lang Tong, Cornell University, United States

SS-L10.4: AN INNOVATIVE RTP-BASED RESIDENTIAL POWER SCHEDULING SCHEME FOR SMART GRIDS

Chen Chen, Shalinee Kishore, Lawrence Snyder, Lehigh University, United States

SS-L10.5: FAST ESTIMATION OF THE STATE OF THE POWER GRID USING 5960 SYNCHRONIZED PHASOR MEASUREMENTS

Tao Yang, Anjan Bose, Washington State University, United States

SS-L10.6: DIRECT LOAD MANAGEMENT OF ELECTRIC VEHICLES 5964 Mahnoosh Alizadeh, Anna Scaglione, University of California Davis, United States; Robert J. Thomas, Cornell University, United States

\section{SS-L11: COMPRESSED SENSING AND SPARSE REPRESENTATION OF SIGNALS}

SS-L11.1: BEATING NYQUIST THROUGH CORRELATIONS: A CONSTRAINED RANDOM 5968 DEMODULATOR FOR SAMPLING OF SPARSE BANDLIMITED SIGNALS

Andrew Harms, Princeton University, United States; Waheed U. Bajwa, Robert Calderbank, Duke University, United States

SS-L11.2: COMPRESSIVE SENSING FOR OVER-THE-AIR ULTRASOUND 5972

Petros Boufounos, Mitsubishi Electric Research Laboratories, United States

SS-L11.3: SPARSE SPECTRAL FACTORIZATION: UNICITY AND RECONSTRUCTION 5976 ALGORITHMS

Yue Lu, Harvard University, United States; Martin Vetterli, Ecole Polytechnique Fédérale de Lausanne, Switzerland

SS-L11.4: RAND PPM : A LOW POWER COMPRESSIVE SAMPLING ANALOG TO DIGITAL 5980 CONVERTER

Praveen Yenduri, Anna Gilbert, Michael Flynn, Shahrzad Naraghi, University of Michigan, United States

SS-L11.5: INCOHERENT COLOR FRAMES FOR COMPRESSIVE DEMOSAICING 5984

Abdolreza Abdolhosseini Moghadam, Mohammad Aghagolzadeh, Michigan State University, United States; Mrityunjay Kumar, Eastman Kodak Company, United States; Hayder Radha, Michigan State University, United States

SS-L11.6: IMPROVED THRESHOLDS FOR RANK MINIMIZATION 5988

Samet Oymak, M. Amin Khajehnejad, Babak Hassibi, California Institute of Technology, United States

\section{SS-L12: SYSTEMS BIOLOGY}


Cécile Bazot, Nicolas Dobigeon, Jean-Yves Tourneret, University of Toulouse, France; Alfred O. Hero III, University of Michigan, United States

SS-L12.3: A STOCHASTIC COMPARTMENTAL APPROACH TO MODELING AND 6000 SIMULATION OF CANCER SPHEROID FORMATION AND EVOLUTION

Monica Bugallo, Shishir Dash, Galina Botchkina, Stony Brook University, United States; Marco Lops, INPT/IRIT/ENSEEIHT, France; Petar Djuric, Stony Brook University, United States

SS-L12.5: FAST NETWORK QUERYING ALGORITHM FOR SEARCHING LARGE-SCALE 6008 BIOLOGICAL NETWORKS

Sayed Mohammad Ebrahim Sahraeian, Byung-Jun Yoon, Texas A\&M University, United States

SS-L12.6: UNCOVER COOPERATIVE GENE REGULATIONS BY MICRORNAS AND 6012 TRANSCRIPTION FACTORS IN GLIOBLASTOMA USING A NONNEGATIVE HYBRID FACTOR MODEL

Jia Meng, The University of Texas at San Antonio, United States; Hung-I Chen, The University of Texas Health Science Center at San Antonio, United States; Jianqiu Zhang, The University of Texas at San Antonio, United States; Yidong Chen, The University of Texas Health Science Center at San Antonio, United States; Yufei Huang, The University of Texas at San Antonio, United States 


\title{
FORENSIC VOICE COMPARISON WITH SECULAR SHIBBOLETHS - A HYBRID FUSED GMM-MULTIVARIATE LIKELIHOOD RATIO-BASED APPROACH USING ALVEOLO-PALATAL FRICATIVE CEPSTRAL SPECTRA
}

\author{
Phil Rose \\ School of Language Studies, Australian National University
}

\begin{abstract}
The suitability of voiceless fricative spectra for forensic voice comparison is explored within a Likelihood Ratio-based framework. Non-contemporaneous landline telephone recordings of 99 male Japanese speakers are compared using only tokens of their voiceless alveolo-patalal fricative [c]. A subset of meancepstrally-subtracted LPC CCs from the fricative spectrum from dc to $5 \mathrm{kHz}$ is used. GMM/UBM and multivariate likelihood ratios are extracted for the 99 target and 4851 non-target trials, and fused with logistic regression. An EER of $7.4 \%$ and $\log$-LR cost of 0.26 is demonstrated. It is concluded that the [6] spectrum does have some individualising potential.
\end{abstract}

Index Terms - Forensic Voice Comparison, GMM/UBM, Multivariate Likelihood Ratio, coronal fricative spectra, cepstrum.

\section{INTRODUCTION}

In the last decade, forensic speaker recognition has experienced a remarkable paradigm shift [9]. Built around the likelihood ratio (LR) of Bayes' Theorem, approaches have been developed with both automatic speaker recognition and traditional phonetic methods which now make it possible to claim [5] emulation, at least in methodology, of what is now considered the gold-standard of forensic identification-of-the-source sciences, namely DNA profiling [2]. In Spain and Australia, LR-based forensic voice comparison evidence has also now been received in court.

As currently practiced, traditional and automatic LR-based forensic voice comparison (FVC) differ in several ways: in the use of generative $v s$ discriminative LRs; the amount of processable information and the time required to complete an analysis; sensitivity to channel effects; and the potential strength of evidence achievable. The main difference, however, is in the information used. Traditional approaches are essentially local in both time and frequency domain, with traditional phonetic features more closely associated with linguistic units, like formant centre frequencies extracted from linguistically comparable items, e.g. the same word or phoneme. In traditional FVC the expert first scours the suspect and offender samples for comparable linguistic units, like vowel phonemes. The traditional acoustic properties of these units, e.g. formant centre-frequencies, are then quantified and LRs estimated. LRs for the different linguistic units are then combined to obtain an overall LR, e.g. [15].

The most commonly used traditional LR-based FVC feature is vowel formant centre-frequencies, in real case-work usually parametrised as point estimates of so-called formant target values. Their individualising potential has been fairly extensively tested e.g. [8]. Language contains many speech sounds other than vowels, however, and their forensic potential remains largely unexplored. In this paper I investigate the forensic potential of a segment from another major class of speech sounds - voiceless coronal fricatives (coronal means made by the tip and/or blade of the tongue). According to the Old Testament, these sounds were involved in possibly the earliest speaker recognition experiment, with dire consequences for many [10], but my choice here is motivated by a real-world case involving an AU\$150 million telephone fraud which went to trial in 2008 [11]. The incriminating recording contained little offender speech, but it was highly comparable with the suspect samples in the phrase not too bad, and several tokens of the word yes, and I was able to derive a multivariate LR from this material based on vocalic F-pattern and intonational F0.

One conspicuous feature which I was unable to exploit, other than with a crude categorical LR, was the obvious similarity between offender and suspect /s/ spectra, both of which had a lower than normal cut-off frequency. The lack of a proper methodology for deriving LRs from fricatives, which are common speech sounds, suggested that this was an important FVC research question. This paper thus asks: what strength of FVC evidence can be expected from the spectral features of voiceless fricatives like [s] or [S]? On the one hand, one expects coronal fricative spectra to have a certain amount of individualising potential by virtue of an advantageous between- to within-variance ratio arising from between-speaker differences in the dimensions of the rigid structures involved in their articulation - dentition, alveolar ridge, hard palate - provided that speakers have not lost teeth or had new dentures in the interim. On the other hand, these sounds' most well-defined spectral prominences are located in frequency regions likely to be compromised by telephone channel bandpassing.

It is unlikely that this essentially traditional FVC question can be satisfactorily answered without recourse to automatic signal processing methods, and a second aim of my paper is to explore how traditional and automatic methods can be profitably combined. In order to do this, some common features and backend processing are recruited from automatic forensic speaker recognition. This paper will assess the FVC potential of just one type of voiceless coronal fricative: the alveolo-patalal (also called palatalized post-alveolar) [6] in Japanese.

\section{VOICELESS ALVEOLO-PALATAL FRICATIVE}

The voiceless alveolo-patalal fricative [c] is auditorily similar to the English so-called palato-alveolar sibilant fricative [S] in sheet, but its constriction is further back, with a longer, narrower channel formed from a higher tongue body and blade [7]. The X-rays of [6] in figure 1 show this fairly long palatal channel formed between 
the antero-dorsum of the tongue and the middle of the hard palate. This results in a short front cavity and a much longer back cavity. Similar articulations can be seen in the nice sagital MRIs in [17]. Information on the spectral characteristics of [6] can be found in the acoustic theory of speech production modeling in [16], and the estimation, in [17], of transfer functions from MRI-derived area functions of two Polish speakers'/ $/$ /, compared with mean spectra of their actual productions. The important spectral characteristic of [6] is a compact prominence located between ca. 2.5 and $4.0 \mathrm{kHz}$. This prominence is contributed by the $\lambda / 2$ resonance of the palatal channel, tuned-up somewhat by its finite constriction and posterior flaring, and the $\lambda / 4$ resonance from the front cavity, tuned up by its tapered shape and the non-finite impedance of the palatal channel. Below this main prominence the supralaryngeal articulation predicts two further poles. One is the $\lambda / 2$ resonance associated with the back cavity (if of a sufficiently high amplitude to be spectrographically visible, this should be continuous with vocalic F2). Below that will be a Helmholz resonance which will be continuous with vocalic F1, but below the dynamic range in a normal spectrogram. A $17.5 \mathrm{cms}$. long vocal tract, with a constricted, ca. $4.5 \mathrm{cms}$. palatal channel and a $3 \mathrm{cms}$. front cavity will thus have poles at about $1.8 \mathrm{kHz}$ (rear cavity), $2.9 \mathrm{kHz}$ (front cavity) and $3.8 \mathrm{kHz}$ (palatal channel). Zeros are also expected from the supralaryngeal configuration, mostly from the location of a source at the teeth. In addition to the supralaryngeal articulation, contributions are also expected as a consequence of the laryngeal gesture associated with voicelessness, namely abducted vocal cords. An open glottis means acoustic coupling with the trachea, and one would therefore also expect low frequency spectral peaks (for a male, the lowest just below ca. $1 \mathrm{kHz}$ ), and zeros, from the sub-glottal system.

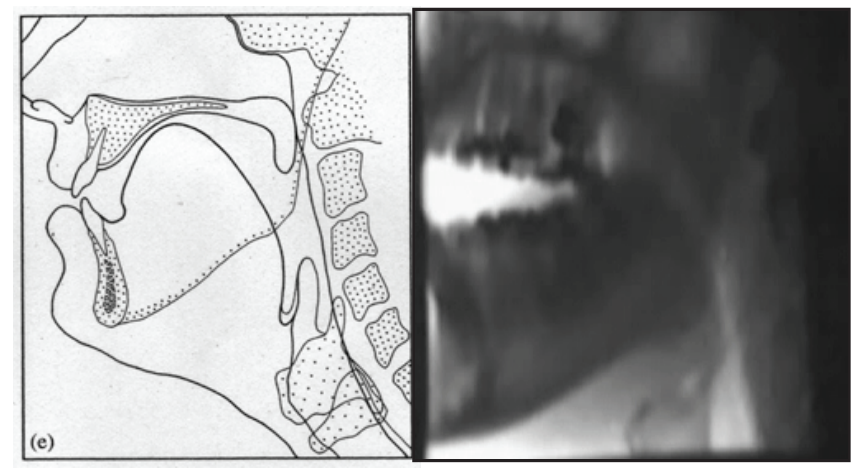

Figure 1. Left: mid-sagital section of tongue position in medial phase of alveolo-palatal fricative [6] estimated from X-ray (reproduced from [6]). Right: X-ray movie of [a'ca] produced by author at the former Adelaide Childrens' Hospital. Click on figure to view movie.

Some of these features can be seen in the conventional wideband spectrogram in figure 2, of the Japanese word kaisha [kaica] company, said over the phone. The band-limiting of the telephone channel is clear, especially in the high frequency region, where no signal energy is evident above $4 \mathrm{kHz}$. The effect in the low frequencies is clear from the up-shifted F1 centre-frequency in the part corresponding to [i] - it is about $450 \mathrm{~Hz}$, whereas one would expect a value closer to 250 or 300 . The portion corresponding to the [c] is clear, lasting about $10 \mathrm{csec}$., from ca. csec. 32 to csec. 44 . It is dominated by narrowband energy constituted from two poles, from front cavity and palatal channel, at ca. 3.0 and $3.5 \mathrm{kHz}$ (due to apparent variable formant-cavity association [17] one cannot say which pole reflects which cavity). There is also weaker energy centered at about $700 \mathrm{~Hz}$ from a sub-glottal resonance, and even weaker energy at $1.8 \mathrm{kHz}$ from the rear cavity. Of note is the clear downwards perturbation at the end of the $3.0 \mathrm{kHz}$ resonance as the tongue moves from [6] to [a], and the drop in noise amplitude immediately preceding [a] periodicity which reflects removal of constriction with cords still abducted.

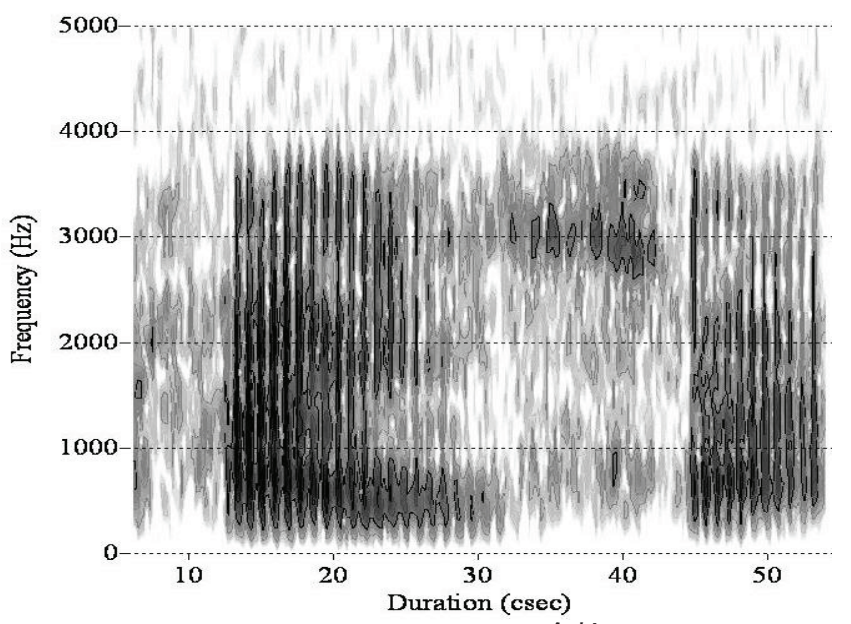

Figure 2. Spectrogram of Japanese word 会社 /kaica/ company, showing acoustics of alveolo-palatal fricative. Listen here.

\section{PROCEDURE}

\subsection{Recording, speakers, corpus.}

Tokens of [6] were taken from the first 99 speakers of a Japanese National Research Institute of Police Science database. This database contains recordings, digitized at $10 \mathrm{kHz}$ with 12 bit quantization, of 297 adult male Japanese from 11 different prefectures around Japan. All speakers were members of the Japanese Police Force and were uncontrolled for age (which ranged from ca. 20 to 50 years). The recordings were made centrally, on the same equipment, of landline telephone calls. Most importantly, two non-contemporaneous recordings were made for each speaker, separated by three to four months. Each recording for each speaker contains about 70-80 seconds net speech comprising two repeats of the set of five Japanese vowels, and several single-word and many-word utterances like kodomo wa daijōbu da the kid's safe, or bakudan o shikaketa I've planted a bomb.

The words and expressions from which the [6] tokens were extracted are given in table 1, together with typical phonetic realisations (typical, because speakers do not invariably conform to prescribed Standard Japanese phonological behavior, and in real speech there is actually considerable between- and within-speaker variation). As can be seen, there were nine tokens of $[6]$ per repeat, seven occurring as the realisation of the palatal fricative phoneme /6/ (romanised as 'sh'), and two as part of the realisation of the palatal affricate phoneme /t $6 /$ (romanised as 'ch'). As there were two repeats per recording session, there were 18 tokens of [c] in each recording. The long [6:] tokens occur as the result of so-called vowel devoicing, whereby high vowels in unaccented syllables become voiceless between two voiceless segments (the end of a word is also considered voiceless). When this occurs, the constriction for a preceding [6] is simply continued for the 
duration of the devoiced vowel. Thus in ashita /acita ${ }^{7} /$ tomorrow, the high vowel /i/ occurs in an unaccented syllable between two voiceless consonants $/ \varphi /$ and $/ t /$, and so devoices. The constriction of the $/ \zeta /$ is then prolonged, resulting in a long fricative segment [6:] occurring before a [t]. As a result of devoicing, the [6] actually occurs in a variety of contexts, before [i a t k], and after [a i o t]. This contributes some within-speaker variation, as the [6] assimilates a little to its surroundings.

\begin{tabular}{|c|c|c|c|}
\hline もしもし & hello & moshimoshi & mocimoci \\
\hline 私 & me & watashi & wataci \\
\hline 会社 & company & kaisha & kaica \\
\hline $\begin{array}{c}\text { 爆弾を } \\
\text { 仕掛けた }\end{array}$ & $\begin{array}{c}\text { I've planted } \\
\text { a bomb }\end{array}$ & $\begin{array}{l}\text { bakudan o } \\
\text { shikaketa }\end{array}$ & o crkaketa \\
\hline 明日の朝 & $\begin{array}{c}\text { tomorrow } \\
\text { morning }\end{array}$ & ashita no asa & acita \\
\hline $\begin{array}{c}\text { 金を用意 } \\
\text { しろ }\end{array}$ & $\begin{array}{l}\text { get the } \\
\text { money } \\
\text { ready }\end{array}$ & $\begin{array}{c}\text { kane o } \\
\text { yōishiro }\end{array}$ & jo:iciro \\
\hline 一 & one & ichi & itc: \\
\hline 八 & eight & hachi & hatc: \\
\hline
\end{tabular}

\subsection{Front-end processing}

Utterances containing [6] were located and saved as separate audio files. Conventional wideband spectrograms of the utterances with superimposed intensity contours and formants (Burg, six formants below $5 \mathrm{kHz}$ ) were generated with Praat, and a spectrally maximally homogeneous portion of [G] selected by eye. This portion was then saved in a separate. wav audio file and $12^{\text {th }}$ order LPC CCs extracted. Cepstral subtraction was then performed using the mean cepstral vector obtained from the speaker's whole repeat. This process is illustrated in figure 3, which shows the cepstral spectrum for the [c] token in figure 2 both before and after mean cepstral subtraction. The band-limiting of the telephone is clear in both the mean cepstral spectrum of the whole repeat and the spectrum of the segment in the left panel. Of interest is the cepstrally-mean-subtracted $(\mathrm{cms})$ spectrum in the right panel, which appears to represent a partially restored typical spectrum for a fricative of this kind. Four peaks are obvious: the two highest amplitude peaks at ca. 2.9 and $3.6 \mathrm{kHz}$ presumably reflect front cavity and palatal channel resonances; the two lowest peaks may reflect sub-glottal and back cavity resonance.

\subsection{Back-end processing}

An optimum set of cms CCs was empirically selected using a version of the multivariate LR formula developed at Edinburgh University's Joseph Bell Centre for Forensic Statistics and Legal Reasoning [1], followed by calibration [12] using Brümmer's Focal toolkit [3]. The CCs were optimized for the calibrated log likelihood ratio cost Cllr and equal error rate. Cllr is currently the evaluation metric of choice for the performance of LR-based detection systems. It is a simple scalar mean of two hypothesisdependent logarithmic functions, one for all target LRs and one for all non-target LRs, which severely penalizes highly misleading
LRs [4]. It was found that a improvement in evaluation of target LR performance was obtained by discarding four cms CCs (4 68 9), and so the remaining six cms CCs were used.
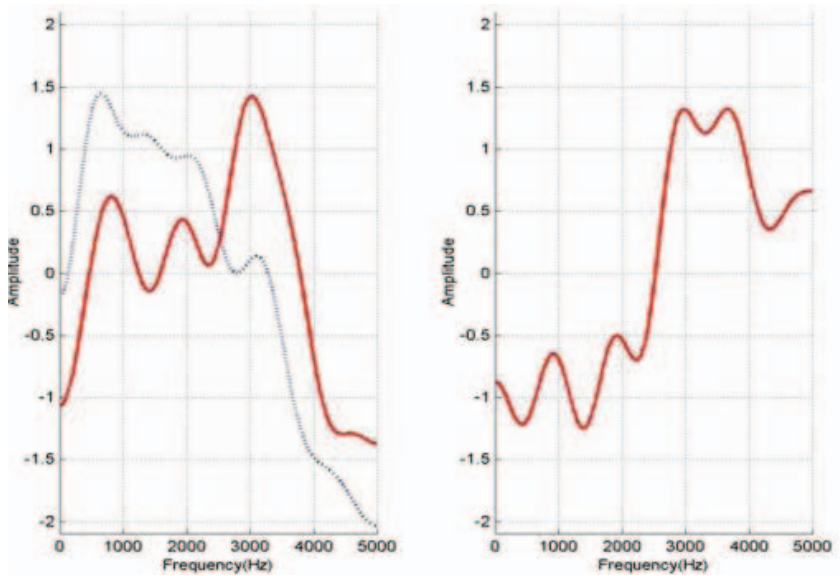

Figure 3. Illustration of cepstral mean subtraction in [6]. Left panel: red line $=12^{\text {th }}$ order LPC cepstral spectrum of phonerecorded [6] in [kaica] from figure 2, dotted line: speaker's mean cepstral spectrum. Right panel: cepstrally-mean-subtracted cepstral spectrum for $[6]$.

LR-based forensic voice comparison was then performed on the 99 speakers' non-contemporaneous [6] cms CCs using both multivariate (MV) and GMM/UBM likelihood ratios [13]. With two non-contemporaneous recordings, two independent non-target trials are possible. Only one was used - between speakers' first recording sessions - thus giving 99 target and 4851 non-target trials.

\section{RESULTS}

Figure 4 shows, by means of the now conventional Tippett, or reliability plot, the results of the FVC using both $\mathrm{MV}$ and GMM/UBM LRs. Although both show an EER of ca. 10\% and also have effectively the same calibrated Cllr values (given in the figure), their overall performances differ considerably, involving a quasi $20^{\circ}$ rotation around threshold. (This brings into question the adequacy of Cllr as a metric, of course.) The multivariate analysis is clearly superior to the GMM in non-target trial resolution: half the non-target MV LRs are smaller than ca. $\log 10-2$, compared to $\log 10-1$ for the GMM LRs. More importantly, the maximum counterfactual non-target MV LR is ca. $\log 10 \mathrm{LR}=2$, compared to just under $\log 10 \mathrm{LR}$ of 5 for GMM. From a legal perspective (avoid convicting the innocent rather than exonerating the guilty) the higher GMM values are clearly undesirable. The superiority is reversed for target trials, at least as far as the higher LR values are concerned. The performance of MV and GMM systems is almost identical for the lowest $50 \%$ of the cases, with the worst counterfactual values remaining nicely below $\log 10 \mathrm{LR}=\mathrm{ca} .-1.5$. The GMM/UBM is clearly capable of very much higher strength of evidence for the upper $50 \%$ of target trials.

FVC is ultimately about estimating the strength of voice evidence in a specific case. Despite the encouraging similarity in the EER and Cllr of the MV and GMM/UBM results, the individual LR estimates of the two approaches differ in many respects, and, since Cllr indicates no obvious preference, it is best to fuse rather than chose. As shown in figure 5, the result is an 
overall improvement: Cllr reduces to 0.26 , EER to ca. $7.4 \%$. The magnitude of the worst counterfactual non-target LRs remains a worry, however.

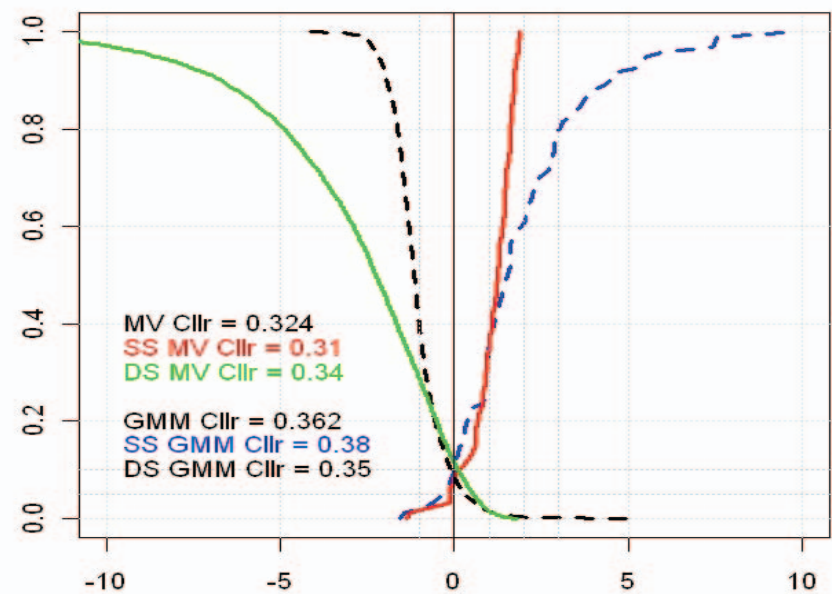

Figure 4. Tippett plot for multivariate (solid) and GMM/UBM (dashed) LRs derived from comparisons using $\mathrm{cms}$ LPC CCs from [c]. $\mathrm{X}$ axis $=\log 10 \mathrm{LR}$ greater than ...; Y axis = proportion of nontarget trials $\sim 1$ /proportion of target trials. $\mathrm{SS}=$ target, $\mathrm{DS}=$ nontarget trials.

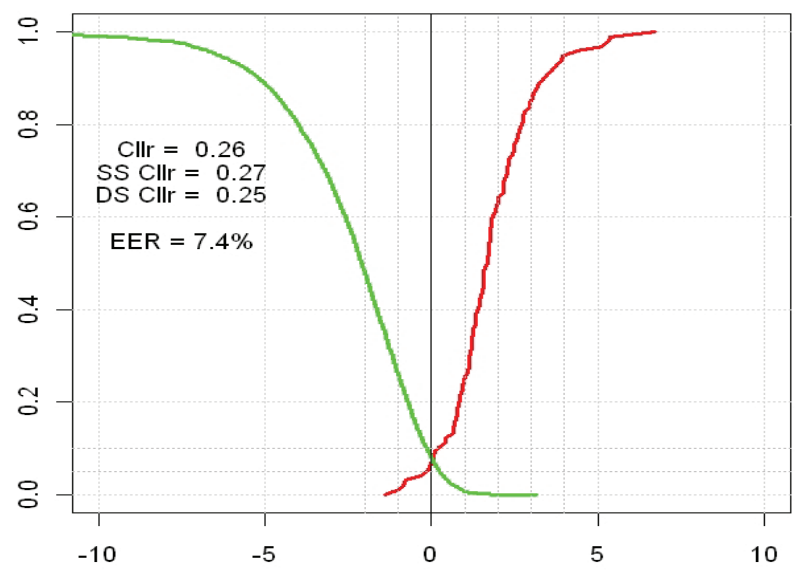

Figure 5. Tippett plot for logistic-regressively fused MV \& GMM/UBM LRs derived from comparisons using meancepstrally-subtracted LPC CCs from [6]. X \& Y axes as for fig. 4.

\section{SUMMARY \& CONCLUSION}

A part traditional, part automatic, FVC procedure has been described using spectra from voiceless alveolo-palatal fricatives. By fusing already reasonable LR estimates from both GMM/UBM and MV approaches, it has shown that [6] is likely to be of use in FVC when its LR is combined with LRs from other segments. Other common coronals, e.g. [s] and [S], should now be tested, and refinements to the method sought, in particular focusing on improved channel compensation, band-limited cepstra, and alternative features (e.g. MFCCs, spectral peak frequencies).

\section{ACKNOWLEDGEMENTS}

This paper was written as part of Australian Research Council Discovery Grant No. DP0774115. Many thanks, but no criticism, are due to Drs. Osanai, Clermont, Kinoshita for advice and help in processing. Thanks also to my four reviewers, one of whose advice I was able to take to increase my subjects (from an original 75).

\section{REFERENCES}

[1] C.G.G. Aitken and D. Lucy. "Evaluation of trace evidence in the form of multivariate data", Applied Statistics 53/4, pp. 109$122,2004$.

[2] D.J. Balding, Weight of Evidence for Forensic DNA Profiles, Wiley, Chichester, 2005.

[3] N. Brümmer, "Focal Toolkit" http://www.dsp.sun.ac.za/nbrummer/focal

[4] N. Brümmer and J. du Preez, "Application independent evaluation of speaker detection", Computer Speech and Language 20/2-3, pp. 230-275, 2006.

[5] J. Gonzalez-Rodriguez, P. Rose, D. Ramos, D. Torre and J. Ortega-García, "Emulating DNA: Rigorous Quantification of Evidential Weight in Transparent and Testable Forensic Speaker Recognition", IEEE Transactions on Audio Speech and Language Processing 15/7, pp. 2104 - 2115, 2007.

[6] J.M.D. Laver, Principles of Phonetics, University Press, Cambridge UK, 1994.

[7] P. Ladefoged and I. Maddieson, Sounds of the World's Languages, Blackwell, Oxford, 1996.

[8] G.S. Morrison, "Likelihood Ratio forensic voice comparison using parametric representation of the formant trajectories of diphthongs", JASA 125, pp. 2387-2397, 2009.

[9] G.S. Morrison, "Forensic voice comparison and the paradigm shift”, Science \& Justice 49, pp. 298-308, 2009.

[10] Old Testament, Judges 12: 5,6.

[11] R v Hufnagl, Ernst [2008] NSWDC. See also http://www.abc.net.au/pm/content/2008/s2451596.htm

[12] D. Ramos-Castro, J. Gonzalez-Rodriguez and J. OrtegaGarcia, "Likelihood Ratio Calibration in a transparent and Testable Forensic Speaker Recognition Framework", Proc. IEEE Odyssey, 2006.

[13] D.A. Reynolds, T.F. Quaterieri and R.B. Dunn, "Speaker Verification Using Adapted Gaussian Mixture Models", Digital Signal Processing 10, pp. 19-41, 2000.

[14] P. Rose, "Technical Forensic Speaker Recognition: Evaluation, Types and Testing of Evidence", Computer Speech and Language 20/2-3, pp. 159-191, 2006.

[15] P. Rose, "The Effect of Correlation on Strength of Evidence Estimates in Forensic Voice Comparison: Uni- and Multivariate Likelihood Ratio-based Discrimination with Australian English Vowel Acoustics", International Journal of Biometrics 2/14, pp. 316-329, 2010.

[16] K.N. Stevens, Acoustic Phonetics, MIT Press, Cambridge Mass., 1998.

[17] M. Toda, S. Maeda and K. Honda, "Formant-cavity affiliation in sibilant fricatives". In S. Fuchs, M. Toda, M. Żygis (eds.) Turbulent Sounds, Mouton De Gruyter, New York, pp. 343-374, 2010. 